U.S.Aruenpoast. Eng. Res. Ef IP

TP 77-11

\title{
Forces Exerted by Waves on a Pipeline At or Near the Ocean Bottom
}

by

George L. Bowie

TECHNICAL PAPER NO. 77-11 OCTOBER 1977
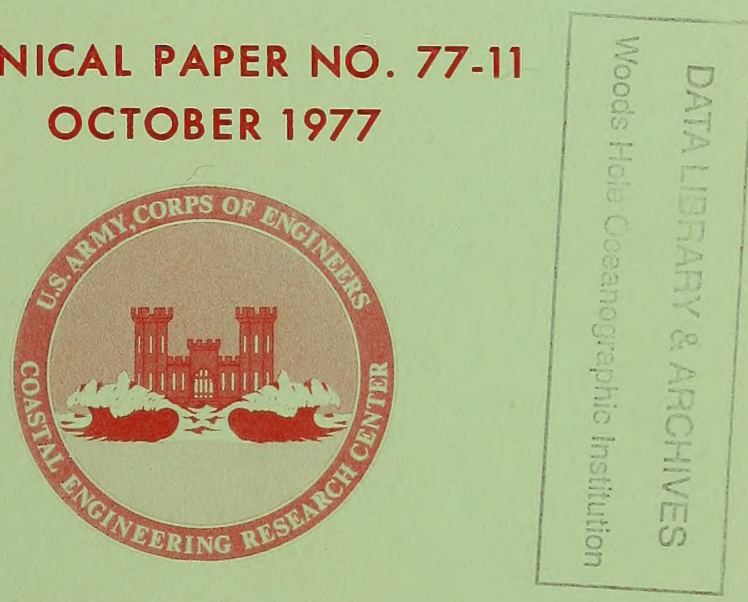

Approved for public release; distribution unlimited.

Prepared for

U.S. ARMY, CORPS OF ENGINEERS

COASTAL ENGINEERING

Kingman Building

Fort Belvoir, Va. 22060 
Reprint or republication of any of this material shall give appropriate credit to the U.S. Army Coastal Engineering Research Center.

Limited free distribution within the United States of single copies of this publication has been made by this Center. Additional copies are available from:

\section{National Technical Information Service ATTN: Operations Division 5285 Port Royal Road \\ Springfield, Virginia 22151}

Contents of this report are not to be used for advertising, publication, or promotional purposes. Citation of trade names does not constitute an official endorsement or approval of the use of such commercial products.

The findings in this report are not to be construed as an official Department of the Army position unless so designated by other authorized documents. 


\begin{tabular}{|c|c|c|}
\hline \multicolumn{2}{|r|}{ REPORT DOCUMENTATION PAGE } & $\begin{array}{c}\text { READ INSTRUCTIONS } \\
\text { BEFORE COMPLETING FORM } \\
\end{array}$ \\
\hline & \begin{tabular}{l|l|} 
REPORT NUMBER & 2. GOVT ACCESSION NO. \\
TP $77-11$ & \\
\end{tabular} & 3. RECIPIENT'S CATALOG NUMBER \\
\hline \multirow{2}{*}{\multicolumn{2}{|c|}{$\begin{array}{l}\text { 4. TITLE (and Subttle) } \\
\text { FORCES EXERTED BY WAVES ON A PIPELINE } \\
\text { AT OR NEAR THE OCEAN BOTTOM }\end{array}$}} & $\begin{array}{l}\text { 5. TYPE OF REPORT \& PERIOD COVERED } \\
\text { Technical Paper }\end{array}$ \\
\hline & & $\begin{array}{l}\text { 6. PERFORMING ORG. REPORT NUMBER } \\
\text { Technical Report HEL 9-24 }\end{array}$ \\
\hline \multicolumn{2}{|r|}{$\begin{array}{l}\text { 7. AUTHOR(s) } \\
\text { George L. Bowie }\end{array}$} & $\begin{array}{l}\text { 8. CONTRACT OR GRANT NUMBER(s) } \\
\text { DACW72-74-C-0004 }\end{array}$ \\
\hline \multicolumn{2}{|r|}{$\begin{array}{l}\text { 9. PERFORMING ORGANIZATION NAME AND ADDRESS } \\
\text { University of California } \\
\text { Hydraulic Engineering Laboratory } \\
\text { Berkeley, California } 94720\end{array}$} & $\begin{array}{l}\text { 10. PROGRAM ELEMENT. PROJECT, TASK } \\
\text { AREA \& WORK UNIT NUMBERS } \\
\text { F31234 }\end{array}$ \\
\hline \multirow{4}{*}{\multicolumn{2}{|c|}{$\begin{array}{l}\text { 11. CONTROLLING OFFICE NAME AND ADDRESS } \\
\text { Department of the Army } \\
\text { Coastal Engineering Research Center (CEREN-DE) } \\
\text { Kingman Building, Fort Belvoir, Virginia 22060 } \\
\text { 14. MONITORING AGENCY NAME ADDRESS(if different from Controlling Office) }\end{array}$}} & $\begin{array}{l}\text { 12. REPORT DATE } \\
\text { October } 1977 \\
\end{array}$ \\
\hline & & $\begin{array}{l}\text { 13. NUMBER OF PAGES } \\
177\end{array}$ \\
\hline & & $\begin{array}{l}\text { 15. SECURITY CLASS. (of this report) } \\
\text { UNCLASS IF IED }\end{array}$ \\
\hline & & $\begin{array}{l}\text { 15a. DECLASSIFICATION/DOWNGRADING } \\
\text { SCHEDULE }\end{array}$ \\
\hline \multirow{2}{*}{\multicolumn{3}{|c|}{$\begin{array}{l}\text { 16. DISTRIBUTION STATEMENT (of this Report) } \\
\text { Approved for public release; distribution unlimited. }\end{array}$}} \\
\hline & & \\
\hline \multicolumn{3}{|c|}{ 17. DISTRIBUTION STATEMENT (of the abstract entered in Block 20, if different from Report) } \\
\hline \multicolumn{3}{|c|}{ 18. SUPPLEMENTARY NOTES } \\
\hline \multirow{2}{*}{\multicolumn{3}{|c|}{$\begin{array}{l}\text { 19. KEY WORDS (Continue on reverse side if necessary and ldentlfy by block number) } \\
\text { Submarine pipeline } \\
\text { Two- and three-dimensional experiments } \\
\text { Wave force analysis } \\
\text { Wave-induced lift forces }\end{array}$}} \\
\hline & & \\
\hline \multicolumn{3}{|c|}{ 0. ABSTRACT (Continue on reverse side it necesaary and identify by block number) } \\
\hline \multicolumn{3}{|c|}{$\begin{array}{l}\text { The wave-induced forces on a submarine pipeline near the ocean floor } \\
\text { consist of several components--inertial forces, drag forces, lift forces, } \\
\text { and under some conditions, eddy-induced forces. For a pipeline touching } \\
\text { the bottom, or at a small clearance above the bottom, the lift force is } \\
\text { the predominant force in the vertical direction. This force is generally } \\
\text { expressed as } \mathrm{F}_{\mathrm{L}}=1 / 2 \mathrm{C}_{\mathrm{L}} \rho \mathrm{A} \mathrm{u} \mathrm{u}^{2} \text {, and is added as a lift term to the Morison } \\
\text { equation. } \\
\text { (Continued) }\end{array}$} \\
\hline
\end{tabular}


The experimental results of this investigation, however, show that this steady-flow lift model is inadequate for wave-induced oscillatory flows. For pipelines at small clearances above the bottom, viscous effects near the bottom clearance constriction may result in lift forces acting in both the upward and downward directions during different parts of the wave cycle. In addition, the maximum positive and negative lift forces may not correspond to the positions of maximum horizontal velocities in the wave cycle.

A modified 1ift force model of the form, $F_{L}=1 / 2 C_{L} \rho A u_{m a x} 2$ $\left[\cos ^{2}(\theta-\phi)-k\right]$, is proposed where the parameters, $C_{L}, \dot{\phi}$, and $k$, may vary accordingly to allow adequate description of all characteristics of the lift force phenomenon. Quantitative relationships between these unknown 1ift force parameters and various dimensionless parameters defining the wave and pipe conditions were found. These relationships exhibited good correlation for all wave conditions, bottom clearances, pipe diameters, and orientation angles. 


\section{PREFACE}

This report is published to provide coastal engineers with an analysis of wave-induced forces on a submarine pipeline near the ocean floor. The work was carried out under the structural design program of the U.S. Army Coastal Engineering Research Center (CERC).

The report was prepared by George L. Bowie, Research Assistant, University of California, Berkeley, under CERC Contract No. DACW72-74C-0004.

The author acknowledges the help and advice of Professor R.L. Wiege1, Professor J.W. Johnson, and Dr. J.D. Cumming, throughout different stages of the project. Many thanks also go to Dr. M.F. Al-Kazily, J. Allison, and L. Magel for their help in designing and setting up the instrumentation for the experiments, and to W. Krogmoe, E. Parscale, and W. Matthew for their skillful assistance in building the models.

Dr. J.R. Weggel was the CERC contract monitor, under the general supervision of G.M. Watts, Chief, Engineering Development Division.

Comments on this publication are invited.

Approved for publication in accordance with Public Law 166, 79th Congress, approved 31 July 1945, as supplemented by Public Law 172, 88th Congress, approved 7 November 1963.

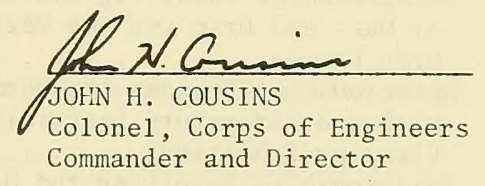


CONVERSION FACTORS, U.S. CUSTOMARY TO METRIC (SI) . . . . . . 9

SYMBOLS AND DEFINITIONSS . . . . . . . . . . . . . 10

I WAVE FORCE ANALYSiS . . . . . . . . . . . . . . . . 17

1. Wave Force Components on Pipelines

Near the Bottom . . . . . . . . . . . . . 17

2. Wave-Induced Lift Forces . . . . . . . . . . . . . 19

3. Model for Wave-Induced Lift Forces . . . . . . . . . 26

4. Extension of Model to Higher Order Theories . . . . . 32

II EXPERIMENTAL INVESTIGATION . . . . . . . . . . . . . 36

1. Experimental Equipment . . . . . . . . . . . . 36

2. Procedure for Two-Dimensional Experiments . . . . . . 45

3. Procedure for Three-Dimensional Experiments . . . . . 51

4. Data Reduction ... . . . . . . . . . . . 53

III RESULTS AND DISCUSSION . . . . . . . . . . . . . . 58

1. Resultant Force Through Wave Cycle . . . . . . . . 58

2. Orientation Angle Considerations . . . . . . . . . . 75

3. Interrelationships Between $\mathrm{C}_{\mathrm{L}}, \phi$, and $\mathrm{k} . . . . .977$

4. Relationships Between $\phi$ and $\mathrm{k}$ and Parameters

Defining the Wave and Pipeline Conditions . . . . . . 89

5. Relationships Between $\phi$ (clear/Dia) and $k$ (clear/Dia)

and Parameters Defining the Wave and Pipeline

Conditions. . . . . . . . . . . . . . . . . . 104

6. Relationships Between the Coefficients of Lift and

Parameters Defining the Wave and Pipeline Conditions. . 109

7. Relationships Between the Lift Forces and Parameters

Defining the Wave and Pipeline Conditions . . . . . . 109

8. Relationships Involving the Vertical Coefficients

of Mass and Drag and the Vertical Inertial and

Drag Forces . . . . . . . . . . . . . . . . . . 111

9. Relationships Between the Horizontal Coefficient of

Mass and Parameters Describing the Wave and

Pipeline Conditions . . . . . . . . . . . . . . . . 111

10. Relationships Involving the Horizontal Coefficient

of Drag . . . . . . . . . . . . . . . . 113

11. Example Problems . . . . . . . . . . . . 113 
CONTENTS

APPENDIX

Page

A LEAST SQUARES ANALYSIS OF EXPERIMENTAL DATA . . . . . . . . . 125

B COMPUTER PROGRAM FOR VERTICAL LEAST SQUARES ANALYSIS (TWO-DIMENSIONAL DATA) . . . . . . . . . . . . . . . 140

C COMPUTER PROGRAM FOR VERTICAL LEAST SQUARES ANALYSIS

(THREE-DIMENSIONAL DATA) . . . . . . . . . . . . . . . 144

D COMPUTER PROGRAM FOR HORIZONTAL LEAST SQUARES ANALYSIS

(TWO-DIMENSIONAL DATA) . . . . . . . . . . . . . . . 149

E TABULATED VERTICAL FORCE DATA FROM TWO-DIMENSIONAL

EXPERIMENTS . . . . . . . . . . . . . . . 153

F TABULATED VERTICAL FORCE DATA FROM THREE-DIMENSIUNAL

EXPERIMENTS . . . . . . . . . . . . . . . . . 158

G TABULATED HORIZONTAL FORCE DATA FROM TWO-DIMENSIONAL

EXPERIMENTS ...................... 176

TABLE

Estimated accuracy of experimental measurements . . . . . . . . . 59

\section{FI GURES}

1 Change in lift with increasing velocity . . . . . . . . . . . . 21

2 Change in lift force with passing wave crest . . . . . . . . . . . 23

3 Lift force phenomenon . . . . . . . . . . . . . . . . 25

4 Change in lift force record for increasing bottom clearance . . . . 27

5 Definition sketch . . . . . . . . . . . . . . . . . . 29

6 Definition of lift force parameters . . . . . . . . . . . . . . 31

7 Comparison of linear and Stokes' third-order theories: Simultaneous shift of lift force record as $\phi$ increases from $0^{\circ}$ to $90^{\circ}$ and $\mathrm{k}$ increases from 0 to 1 with increasing bottom clearance. . . . . . 34

8 Comparison of lift force extreme cases for linear and Stokes' thirdorder theories... . . . . . . . . . . . . . . 35

9 Force meter and support . . . . . . . . . . . . . . 38 


\section{CONTENTS}

FIGURES--Continued

10 Four-inch cylinder mounted in the flume . . . . . . . . . . . . 39

11 Test section (force meter) . . . . . . . . . . . . . 40

12 Test section mounted in position . . . . . . . . . . . . . . 41

13 Test section and force transducer . . . . . . . . . . . . . 42

14 Schematic of pipeline model . . . . . . . . . . . . . . . 43

15 Pipeline model . . . . . . . . . . . . . . . . . . . 44

16 Brush recording instruments . . . . . . . . . . . . . . 46

17 Digitizer and recording instruments . . . . . . . . . . . . . 47

18 Calibration method for two-dimensional experiments . . . . . . 48

19 Experimental arrangement for two-dimensional tests . . . . . . . . 49

20 Calibration method for three-dimensional experiments . . . . . . 52

21 Experimental arrangement for three-dimensional tests . . . . . . 54

22 Definition sketch for three-dimensional experiments . . . . . . . 55

23 Example of data record . . . . . . . . . . . . . . . 57

24 Example of computer output for vertical least squares analysis .................... . . . 60

25 Example of computer output for horizontal least squares analysis... . . . . . . . . . . . . . . . 61

26 Resultant force through wave cycle for 0.001 -foot clearance, 1.85 -second period, and 0.24 -foot height . . . . . . . . . . 62

27 Resultant force through wave cycle for 1/16-inch clearance, 1.86 -second period, and 0.24 foot height . . . . . . . . . . 63

28 Resultant force through wave cycle for $1 / 8$-inch clearance, 1.85 -second period, and 0.25 -foot height . . . . . . . . . 64

29 Resultant force through wave cycle for $3 / 16$-inch clearance, 1.85 -second period, and 0.25 -foot height . . . . . . . . . . 65 


\section{CONTENTS}

\section{FIGURES--Continued}

30 Resultant force through wave cycle for 1/4-inch clearance,

1.86 -second period, and 0.25 -foot height

31 Resultant force through wave cycle for 1 -inch clearance,

1.86 -second period, and 0.24 -foot height . . . . . . . . . 67

32 Resultant force through wave cycle for 2-inch clearance,

1.86 -second period, and 0.25 -foot height . . . . . . . . . 68

33 Change in resultant force with increasing clearance . . . . . . . . 69

34 Resultant force through wave cycle for 1-inch clearance,

1.23 -second period, and 0.3 -foot height . . . . . . . . . 71

35 Resultant force through wave cycle for 0.001 -foot cle irance,

0.95 -second period, and 0.24 -foot height . . . . . . . . . 72

36 Resultant force through wave cycle for 1/16-inch clearance,

0.95 -second period, and 0.24 -foot height . . . . . . . . . 73

37 Resultant force through wave cycle for 2-inch clearance,

0.96 -second period, and 0.25 -foot height . . . . . . . . . . 74

38 Alternative approaches for handling pipeline orientation angles . . 76

$39 \phi$ versus k . . . . . . . . . . . . . . . . . . . 78

$40 \quad \mathrm{C}_{\mathrm{L}}$ versus $\mathrm{k}$. . . . . . . . . . . . . . . . . . 79

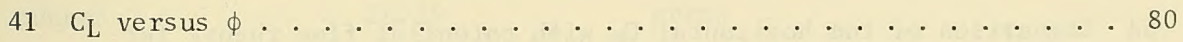

42 Effective positive coefficient of lift versus k . . . . . . . . 82

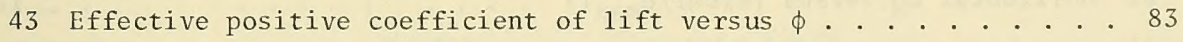

44 Effective negative coefficient of 1 ift versus k . . . . . . . . 84

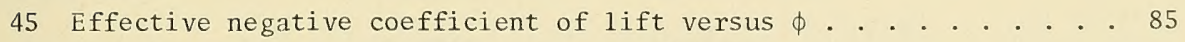

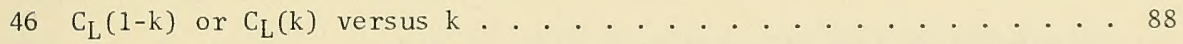

$47 \phi$ versus $\left(\right.$ clear $\left./ \mathrm{u}_{\max } \mathrm{T}\right)$ for 4 -inch diameter . . . . . . . . . . . 91

$48 \phi$ versus $\left(\right.$ clear $\left./ \mathrm{u}_{\max } \mathrm{T}\right)$ for 3 -inch diameter . . . . . . . . . . 92

$49 \phi$ versus $\left(\right.$ clear $\left./ u_{\max } \mathrm{T}\right)$ for 2 -inch diameter . . . . . . . . . . . 93 


\section{CONTENTS}

FIGURES--Continued

$50 \mathrm{k}$ versus $\left(\mathrm{clear} / \mathrm{u}_{\max } \mathrm{T}\right)$ for 4 -inch diameter . . . . . . . . . . . 94

$51 \mathrm{k}$ versus $\left(\mathrm{clear} / \mathrm{u}_{\max } \mathrm{T}\right)$ for 3 -inch diameter . . . . . . . . . . . 95

$52 \mathrm{k}$ versus $\left(\mathrm{clear} / \mathrm{u}_{\max } \mathrm{T}\right)$ for 2 -inch diameter . . . . . . . . . . . 96

$53 \mathrm{k}$ versus $\left(\mathrm{u}_{\max }^{\mathrm{T} / \mathrm{Dia})}\right.$. . . . . . . . . . . . . . . . . . 98

$54 \phi$ versus $\left(\mathrm{u}_{\mathrm{max}} \mathrm{T} / \mathrm{Dia}\right)$. . . . . . . . . . . . . . . . . . . 99

$55 \mathrm{k}$ versus $\left(u_{\max } \mathrm{clear} / \nu\right)$ for 4 -inch diameter . . . . . . . . . . . 100

$56 \phi$ versus $\left(u_{\max } c l e a r / \nu\right)$ for 4 -inch diameter . . . . . . . . . . 101

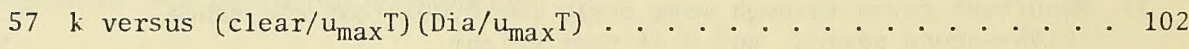

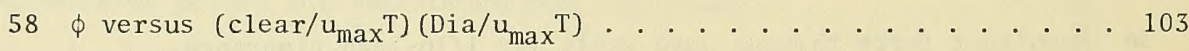

$59 \mathrm{k}(\mathrm{clear} / \mathrm{Dia})$ versus $\left(\mathrm{clear} / \mathrm{u}_{\max } \mathrm{T}\right)(\mathrm{clear} / \mathrm{Dia})$. . . . . . . . . 105

$60 \phi($ clear/Dia $)$ versus $\left(\right.$ clear $\left./ u_{\max } T\right)($ clear/Dia $)$. . . . . . . . . 106

$61 \mathrm{k}(\mathrm{clear} / \mathrm{Dia})$ versus $\sqrt{\mathrm{Dia} / \mathrm{u}_{\max }^{\mathrm{T}}}\left(\mathrm{clear} / \mathrm{u}_{\max } \mathrm{T}\right)(\mathrm{clear} / \mathrm{Dia}) . . . .107$

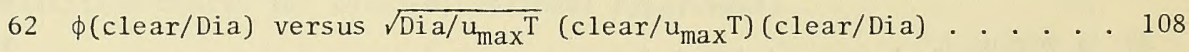

63 Maximum lift force (positive or negative) versus the Reynolds number...................... . . 110

64 Comparison of the horizontal $\mathrm{C}_{\mathrm{M}}$ with potential flow theory for a flow with constant acceleration ............. . 112

65 Horizontal $\mathrm{C}_{\mathrm{M}}$ versus $\left(\mathrm{clear} / \mathrm{u}_{\max } \mathrm{T}\right) \ldots . . . . . . . . . . . . . . . . . .114$ 
U.S. customary units of measurenent used in this report can be converted to metric (SI) units as follows:

\begin{tabular}{|c|c|c|}
\hline Multiply & by & To obtain \\
\hline $\begin{array}{l}\text { inches } \\
\text { square inches } \\
\text { cubic inches }\end{array}$ & $\begin{array}{l}25.4 \\
2.54 \\
6.452 \\
16.39\end{array}$ & $\begin{array}{l}\text { millimeters } \\
\text { centimeters } \\
\text { square centimeters } \\
\text { cubic centimeters }\end{array}$ \\
\hline $\begin{array}{l}\text { feet } \\
\text { square feet } \\
\text { cubic feet }\end{array}$ & $\begin{array}{r}30.48 \\
0.3048 \\
0.0929 \\
0.0283\end{array}$ & $\begin{array}{l}\text { celitimeters } \\
\text { meters } \\
\text { square meters } \\
\text { cubic meters }\end{array}$ \\
\hline $\begin{array}{l}\text { yards } \\
\text { square yards } \\
\text { cubic yards }\end{array}$ & $\begin{array}{l}0.9144 \\
0.836 \\
0.7646\end{array}$ & $\begin{array}{l}\text { meters } \\
\text { square meters } \\
\text { cubic meters }\end{array}$ \\
\hline $\begin{array}{l}\text { miles } \\
\quad \text { square miles }\end{array}$ & $\begin{array}{l}1.6093 \\
259.0\end{array}$ & $\begin{array}{l}\text { kilometers } \\
\text { hectares }\end{array}$ \\
\hline knots & 1.8532 & kilometers per hour \\
\hline acres & 0.4047 & hectares \\
\hline foot-pounds & 1.3558 & newton meters \\
\hline millibars & $1.0197 \times 10^{-3}$ & kilograms per square centimeter \\
\hline ounces & 28.35 & grams \\
\hline pounds & $\begin{array}{l}453.6 \\
0.4536\end{array}$ & $\begin{array}{l}\text { grams } \\
\text { kilograms }\end{array}$ \\
\hline ton, long & 1.0160 & metric tons \\
\hline ton, short & 0.9072 & metric tons \\
\hline degrees (angle) & 0.1745 & radians \\
\hline Fahrenheit degrees & $5 / 9$ & Celsius degrees or Kelvins ${ }^{1}$ \\
\hline
\end{tabular}

To obtain Celsius (C) temperature readings from Fahrenheit (F) readings, use formula: $\mathrm{C}=(5 / 9)(\mathrm{F}-32)$.

To obtain kelvin (K) readings, use formula: $K=(5 / 9)(F-32)+273.15$. 
SYMBOLS AND DEFINITIONS

A

ANG

$\mathrm{C}_{\mathrm{D}}$

$\mathrm{C}_{\mathrm{L}}$

$\mathrm{C}_{\mathrm{L}}^{\prime}$

clear

$\mathrm{C}_{\mathrm{M}}$

d

Dia

F

$\mathrm{F}_{\mathrm{D}}$

$\left(\mathrm{F}_{\mathrm{D}}\right)_{\mathrm{h}}$

$\left.{ }^{\left(F_{D}\right.}\right)_{v}$

$\mathrm{F}_{\mathrm{h}}$

$F_{h}\left(\theta_{i}\right)$

$\mathrm{F}_{\text {I }}$

$\left(\mathrm{F}_{\mathrm{I}}\right)_{\mathrm{h}}$

$\left(\mathrm{F}_{\mathrm{I}}\right)_{\mathrm{V}}$

${ }^{\mathrm{F}}$ L

$F^{\prime}$

$\mathrm{F}_{\text {oh }}\left(\theta_{i}\right)$

$F_{\text {ov }}\left(\theta_{i}\right)$

$\mathrm{F}_{\mathrm{V}}$

$F_{V}\left(\theta_{i}\right)$ projected area of pipe section

orientation angle with respect to wave crests

coefficient of drag

coefficient of 1 ift

coefficient of transverse force due to eddy shedding

bottom clearance

coefficient of mass

stillwater depth

pipe diameter

total wave-induced force

drag force

horizontal component of drag force

vertical component of drag force

horizontal component of total wave force

calculated horizontal force at position $\theta_{i}$ in wave cycle inertial force

horizontal component of inertial force

vertical component of inertial force

lift force

transverse "lift" force due to eddy shedding

observed horizontal force at position $\theta_{i}$ in wave cycle

observed vertical force at position $\theta_{i}$ in wave cycle

vertical component of total wave force

calculated vertical force at position $\theta_{i}$ in wave cycle 


\section{SYMBOLS AND DEFINITIONS--Continued}

$\mathrm{H}$

k

L

$\mathrm{T}$

$t$

u

$u_{\text {nax }}$

V

$\mathrm{v}$

$\mathrm{v}_{\max }$

z

$\partial u / \partial t$

$\partial v / \partial t$

$\theta$

$v$

$\rho$

$\phi$

wave height

negative fraction of lift force cycle

wavelength

wave period

time since last wave crest passed over center of pipe section

horizontal component of water particle velocity if pipeline was absent

maximum horizontal water particle velocity if pipeline was absent

volume of fluid displaced by pipe section

vertical component of water particle velocity if pipeline was absent

maximum vertical water particle velocity if pipeline was absent

vertical distance of center of pipe section above bottom

horizontal component of water particle acceleration if pipeline was absent

vertical component of water particle acceleration if pipeline was absent

$2 \pi t / T=$ position of wave cycle over center of pipe section with respect to time

kinematic viscosity of fluid

mass density of fluid

phase shift of maximum lift forces with respect to wave cycle

\section{Computer Programs}

Input Parameters:

ANGLE orientation angle

C

calibration factor for manual digitizer 
CFD downward force calibration factor

CFU

CL bottom clearance

DF downward force calibration factor

DIA pipe diameter

DN

FI(I) wave force readings

Fo zero point of wave force record

HI (I) wave surface readings

$\mathrm{N}$ number of wave force readings

T wave period

UF upward force calibration factor

UP positive wave (crest) calibration factor

Wo zero point of wave record

$\mathrm{XC} \quad$ length of pipe test section

XF amplification factor for force record

XW amplification factor for wave record

YI(I) wave surface readings

Program Variables:

ANG

orientation angle (in radians)

ANGLE orientation angle (in degrees)

$\mathrm{CDH} \quad$ horizontal coefficient of drag

CDV vertical coefficient of drag

CL bottom clearance 


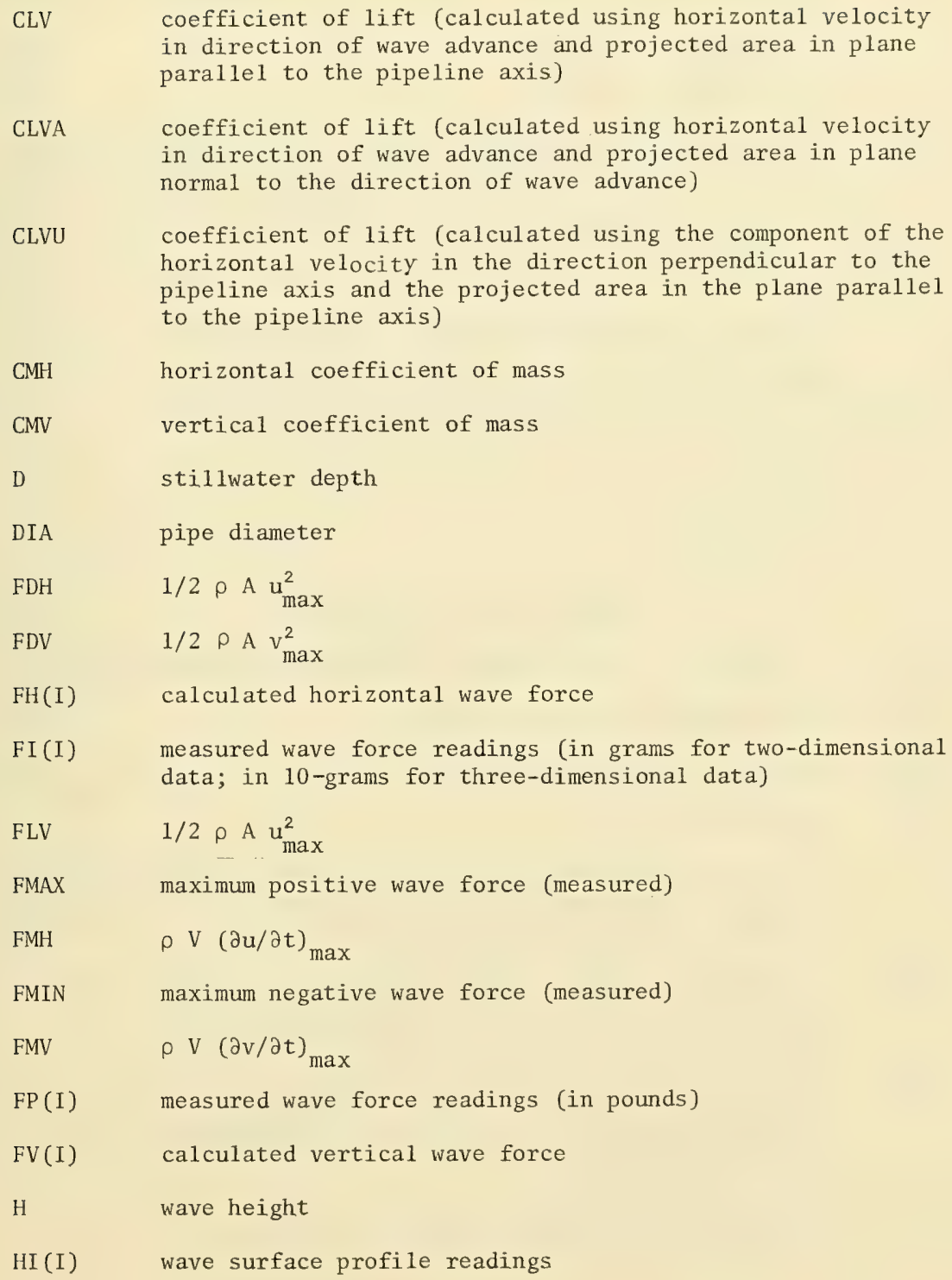


PHI phase-shift parameter $\phi$ of modified lift force equation

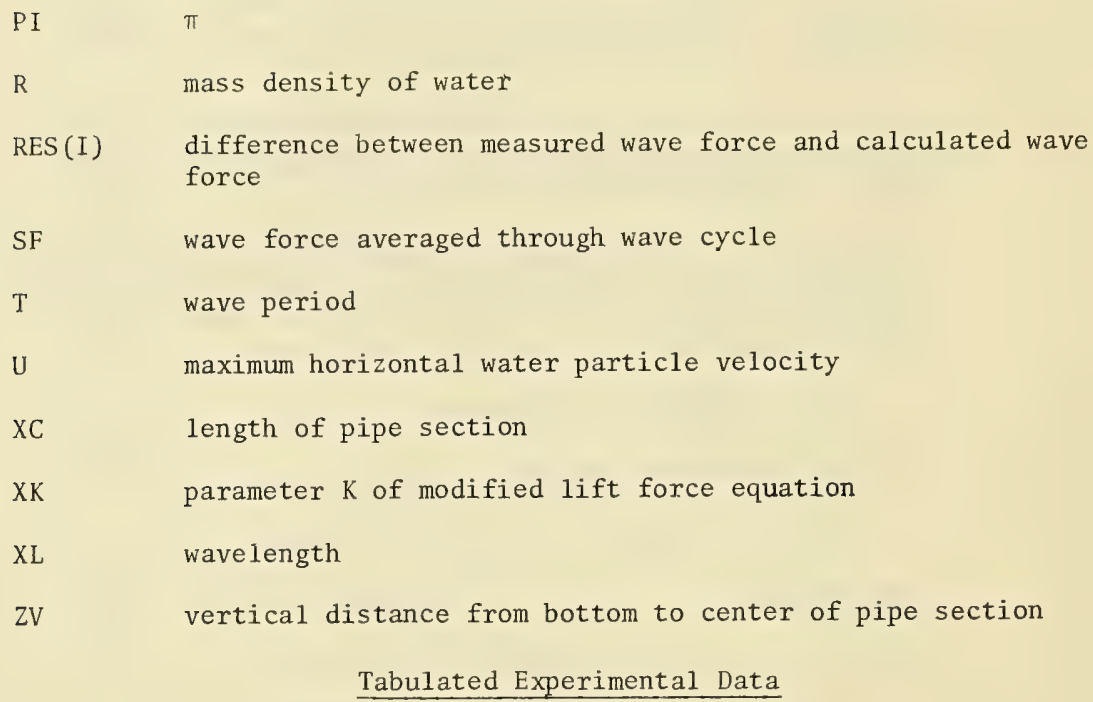

CLVA coefficient of lift (calculated using horizontal velocity in direction of wave advance and projected area in the plane normal to the direction of wave advance)

CLVU coefficient of lift (calculated using the component of the horizontal velocity in the direction perpendicular to the pipeline axis and the projected area in the plane parallel to the pipeline axis)

CMH horizontal coefficient of mass 
vertical coefficient of mass

DIA pipe diameter

FAVG average horizontal force (averaged over complete wave cycle)

H wave height

K. parameter $k$ of modified lift force equation

L wavelength

PHI phase shift parameter $\phi$ of modified lift force equation

T wave period

UMAX maximum horizontal component of water particle velocity at center of pipe section if absent 

FORCES EXERTED BY WAVES ON A PIPELINE

AT OR NEAR THE OCEAN BOTTOM

\section{by \\ George L. Bowie}

\section{WAVE FORCE ANALYSIS}

1. Wave Force Components on Pipelines Near the Bottom.

The most common method of analyzing wave forces on pipelines is the application of the Morison equation (Morison, et a1., 1950). Using this approach, the total wave-induced force on a pipeline can be broken into several components, depending on whether the components are due to the water particle velocities or accelerations. These force components can, in tum, be separated into horizontal and vertical components by using the horizontal and vertical components of the water particle velocities and accelerations in their respective force equations. Where there is no lift effect and no eddy-induced forces, the vertical component, $\mathrm{F}_{\mathrm{V}}$, of the total wave force is

$$
F_{V}=\left(F_{I}\right)_{V}+\left(F_{D}\right)_{V}=C_{M} \rho V \frac{\partial v}{\partial t}+1 / 2 C_{D} \rho A v|v|
$$

and the horizontal component, $F_{h}$, is

$$
F_{h}=\left(F_{I}\right)_{h}+\left(F_{D}\right)_{h}=C_{M} \rho v \frac{\partial u}{\partial t}+1 / 2 C_{D} \rho A u|u|,
$$

where

$$
\begin{array}{ll}
\left(F_{I}\right)_{V} & =\text { vertical component of inertial force } \\
\left(F_{I}\right)_{h} & =\text { horizontal component of inertial force } \\
\left(F_{D}\right)_{V} & =\text { vertical component of drag force } \\
\left(F_{D}\right)_{h} & =\text { horizontal component of drag force } \\
v & =\text { vertical component of water particle velocity if } \\
\mathrm{u} & =\text { pipeline was absent } \\
& \text { if pipeline was absent } \\
\frac{\partial v}{\partial t} & \text { vertical component of water particle acceleration }
\end{array}
$$




$\begin{aligned} \frac{\partial u}{\partial t} & =\quad \begin{array}{l}\text { horizontal component of water particle acceleration } \\ \text { if pipeline was absent }\end{array} \\ \mathrm{A} & =\text { projected area of pipe section } \\ \mathrm{V} & =\text { volume of fluid displaced by pipe section } \\ \rho & =\text { mass density of fluid } \\ \mathrm{C}_{\mathrm{M}} & =\text { coefficient of mass } \\ \mathrm{C}_{\mathrm{D}} & =\text { coefficient of drag }\end{aligned}$

For a pipeline located near the ocean bottom, the water particle orbits are flattened parallel to the boundary. Assuming a horizontal bottom, the vertical motions of the water particles are small in comparison to the horizontal motions, especially in shallow-water depths relative to the wavelength. As a result, the vertical components of the water particle velocities and accelerations are much smaller than the horizontal components, and correspondingly the vertical components of the drag and inertial forces will be smaller than the analogous horizontal forces.

Since the water particles at the bottom are effectively oscillating in a horizontal plane, the vertical excursions of the water particles will generally be less than the diameter of a submarine pipeline lying on or near the bottom. Therefore, the vertical drag forces are generally insignificant, and could probably be neglected from the vertical wave force equation.

Pipelines near the bottom are subject to vertical lift forces. These forces are the result of the asymmetric distortion of the flow field due to the proximity of the bottom boundary, which induces differences in the horizontal flow velocities and corresponding pressure distribution over the top and bottom of the pipeline. Since the water particle velocities near the bottom are at a maximum in the horizontal plane, the lift forces induced by these horizontal motions will generally be the predominant force acting in the vertical direction.

Transverse "lift" forces due to eddy shedding may also be an important component of the vertical wave force, since these forces are also due to the horizontal water particle velocities and excursions which are maximum in the horizontal direction. Certain values of the Keulegan-Carpenter parameter and Reynolds number must be attained for the eddy release phenomenon to occur. The proximity of the bottom boundary will probably have some effect on the formation and release of the eddies, both because it is a solid boundary, and because it affects the orbital motions of the water particles induced by the wave action. 
Although the eddy-induced component of the vertical wave force may be significant when compared to the relatively small vertical drag and inertial forces, the experimental results of this investigation show that the eddy-induced lift forces are much smaller than the "Bernoullitype" lift forces for pipelines located near the bottom. At large clearances above the bottom where the Bernoul1i-type lift effect becomes negligible, the transverse lift forces due to eddy shedding may become a significant component of the total vertical force. At the same time, as the pipeline is raised farther from the bottom boundary, the vertical inertial and drag forces also become more significant.

The vertical component of the total wave-induced force acting on a pipeline near the ocean bottom thus consists of four components--the lift force, the inertial force, the drag force, and the transverse lift force due to eddy shedding. Using the Morison approach, the total vertical wave force is expressed as the sum of these components:

$$
F_{V}=F_{L}+\left(F_{I}\right)_{V}+\left(F_{D}\right)_{V}+F_{L}^{\prime}
$$

where $F_{L}$ is the lift force and $F_{L}{ }^{\prime}$ is the transverse lift force due to eddy shedding.

\section{Wave-Induced Lift Forces.}

Consider a pipeline in contact with a horizontal rigid, impervious bottom. Water cannot flow between the pipe and the bottom boundary, so the flow must be diverted over the top of the pipe. The asymmetrical distortion of the flow field results in maximum velocities over the top of the pipe section and minimum velocities over the bottom, with zero velocities at the stagnation point on the upstream side of the pipe bottom at the point of contact with the sea floor. Correspondingly, the associated pressure distribution will induce an upward lift force for any velocity field acting on the pipeline. The stagnation pressure at the bottom of the pipe section will increase with increasing velocity, while simultaneously the pressure distribution over the top of the pipeline will decrease with the increased velocities of the flow diverted over the top of the pipe section. The wave-induced lift forces will thus act in the upward direction throughout the wave cycle, increasing with the horizontal water particle velocities to maximum magnitudes under the crests and troughs of the passing waves, and diminishing to zero at the points of horizontal flow reversal.

In contrast, a pipeline located at a small clearance above the bottom boundary is subject to a more complex type of lift phenomenon. At the phase in the wave cycle where the horizontal component of the water particle velocity reverses direction, the horizontal velocity over the pipeline is approximately zero. As the wave crest or trough begins to approach the pipeline, the wave-induced horizontal velocities are initially low, inducing unrestricted flow at low velocities over both the top and bottom of the pipeline. However, the water flows 
faster through the bottom clearance constriction than over the top of the pipeline, so the corresponding differences in the pressure distribution exert a downward (negative lift) force toward the bottom boundary (Fig. 1, a).

At first, the negative lift force will increase with the increasing horizontal water particle velocities of the approaching wave, since the flow velocities increase at a faster rate through the bottom clearance constriction than over the top of the pipeline, thus producing larger differences in the corresponding pressure distributions over the top and bottom of the pipe section (Fig. 1, b).

This continues until viscous effects begin to restrict the flow through the narrow bottom clearance. For a given small clearance and a given amount of energy in the horizontal water particle velocities approaching the pipeline, the velocities and flow rates of a viscous fluid through the bottom clearance constriction can attain only certain maximum values. Thus, a "choking" effect is exerted on the restricted flow through the small bottom clearance, and the remainder of the waveinduced flow is forced to flow over the top of the pipe section. Correspondingly, the stagnation point will shift downward, increasing the pressure on the lower upstream side of the pipeline. The larger the proportion of the flow diverted over the top of the pipe, the lower the stagnation point.

At the same time, the increasing velocities associated with the approaching wave crest cause the restricted flow through the bottom clearance to form a turbulent jet with the generation of eddies behind the jet. The generation of increased turbulence and eddies results in an energy loss in the water flowing through the bottom constriction, decreasing the velocities under the pipe section behind the jet.

The above effects associated with the choking phenomenon limit the maximum flow velocities and minimum pressures under the bottom side of the pipe section. In contrast, the unrestricted flow velocities over the top of the pipeline increase freely with the increasing horizontal velocities of the advancing wave. The increased part of the approaching flow that is diverted over the top of the pipe section due to the shift in stagnation point produces a further increase in the flow velocities over the top. Correspondingly, the pressure distribution over the top side of the pipeline decreases at a faster rate than the associated pressures along the bottom side, so the negative lift force gradually decreases and eventually becomes positive (Fig. 1, c, d, and e).

At this stage, the upward lift force becomes larger as the horizontal velocities acting on the pipeline increase further with the advancing wave crest or trough (Fig. 1; f). 
(a)

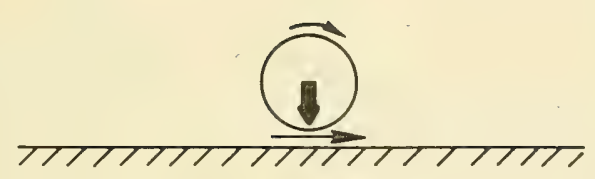

(b)

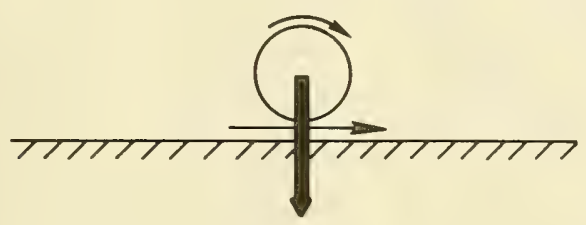

(c)

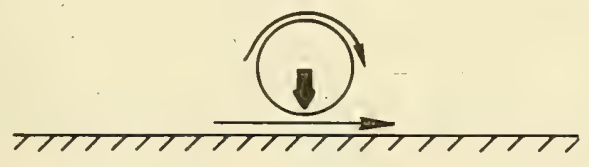

(d)

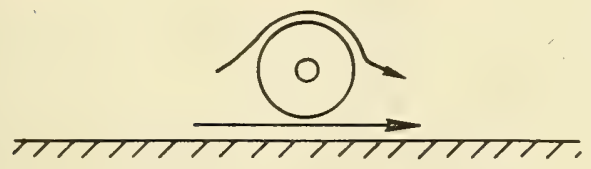

(e)
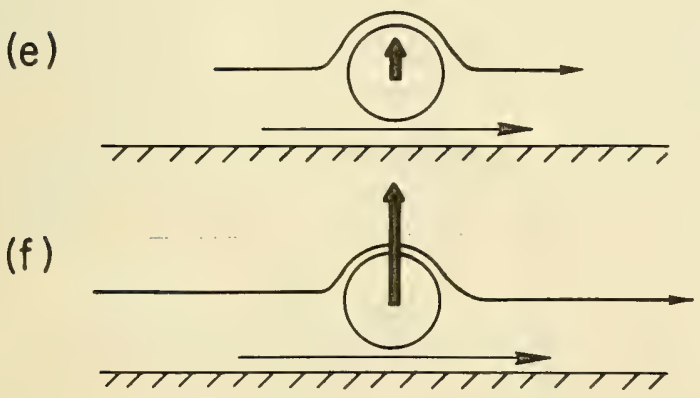

Figure 1. Change in lift with increasing velocity.
SMALL NEGATIVE

LIFT

INCREASING NEGATIVE LIFT

DECREASING NEGATIVE LIFT

ZERO LIFT

SMALL POSITIVE

LIFT

INCREASING POSITIVE LIFT 
As the wave crest or trough passes, this series of steps in the lift force phenomenon is reversed. The horizontal velocities approaching the pipe section begin to decrease, resulting in a decrease in the positive, lift force exerted on the pipeline. As the velocities decrease further with the passing wave, the flow under the pipe section begins to become less restricted. The choking effect thus decreases, and the turbulence and eddies near the bottom clearance gradually diminish. As the flow under the pipe section ceases to be restricted, less of the horizontal flow approaching the pipeline is forced to flow over the top of the pipe, so the stagnation point will accordingly shift upward, closer to the center of the pipe section.

The flow velocities decrease simultaneously over the top and bottom of the pipeline as the wave passes, but the rate of decrease is faster over the top of the pipe than in the vicinity of the bottorn constriction. The positive lift force decreases until eventually, the flow velocities, location of the stagnation point, and associated pressure distribution are such that the pressure integrated over the pipe section again results in a negative lift force. The downward lift force then increases as the flow through the bottom clearance becomes less restricted with the decreasing velocities of the passing wave.

This lift phenomenon, as shown in Figure 2 for a passing wave crest, is repeated twice during each wave cycle as the direction of the waveinduced horizontal velocities reverses under the crests and troughs of the passing waves.

In reality, the horizontal flow reversal occurs almost instantaneously, so the negative lift force does not return to zero at the point of zero velocity when the flow reverses through the bottom clearance constriction. The instant of zero velocity occurs only at the center of the pipe cross section (the reference point). Since the pipeline has a finite diameter, the wave-induced flow acting on the pipe section at any instant includes the sum of the flow conditions induced by the part of the wave covering the entire diameter of the pipeline. So instead of going to zero with the passing wave crest, and then increasing initially with the approaching trough, the lift force remains negative during the period of minimal velocities as the flow reverses under the pipe section.

In a similar manner, the lift force does not become positive as soon as the choking effect occurs in the bottom clearance constriction. The development of the choking phenomenon involves the formation of a turbulent jet through the constriction, and a downward shift in the stagnation point as more water is diverted over the top of the pipe with increasing restriction of the flow through the clearance. The corresponding changes in the velocities, flow pattern, and associated pressure distribution over the top and bottom of the pipe section produce the transition from negative to positive lift. This process requires some small but finite amount of time. Conversely, the reversal 

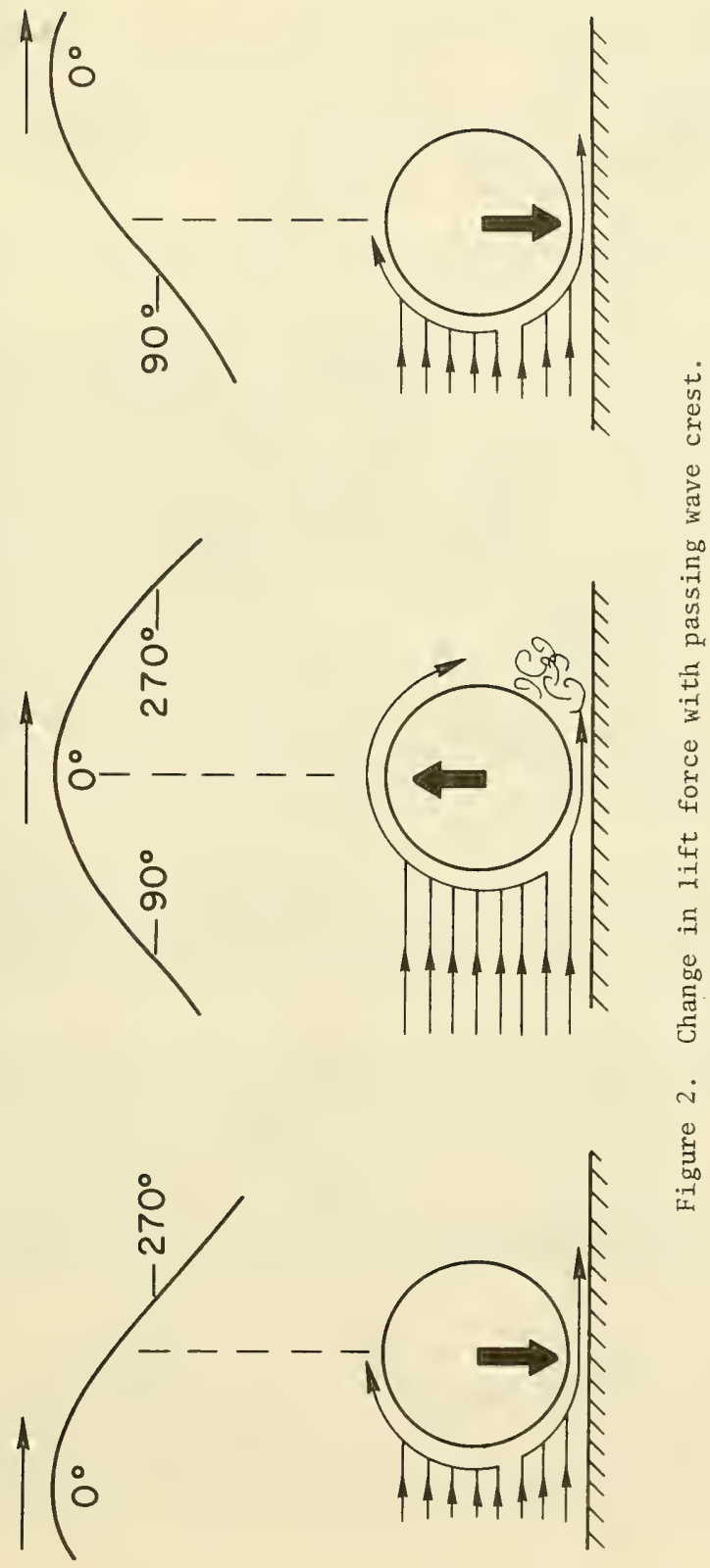
of these processes with the decreasing velocities of the passing wave crest also involves a small but finite amount of time. Thus, there will be a slight timelag in the point of maximum positive lift with reference to the instant of maximum velocity as the wave crest (or trough) passes over the reference point. The smaller the amount of positive lift relative to the amount of negative lift, and the later the positive lift occurs in the wave cycle, the greater the timelag.

An example of the lift force phenomenon over a complete wave cycle for a small bottom clearance is shown in Figure 3 .

For a given pipe diameter and wave condition, as the bottom clearance is increased, higher velocities are necessary to produce the choking effect in which the flow becomes restricted through the bottom clearance constriction. Thus, as the bottom clearance is increased, the flow under the pipeline begins to become restricted closer to the approaching wave crest or trough, where the horizontal velocities are at a maximum; this choking effect also diminishes soon after the wave crest or trough has passed. Therefore, as the bottom clearance is increased, the downward lift force occurs during a larger part of the wave cycle.

At the same time, larger clearances permit greater maximum velocities and corresponding lower pressures under the pipe section. Since higher flow rates are possible under the pipe section, less of the wave-induced flow must be diverted over the top of the pipeline. As a result of these changes, the negative lift forces reach a greater magnitude before the choking effect begins, and these maximums are attained later in the wave cycle.

Correspondingly, the upward lift forces occur during a smaller part of the wave cycle, and the maximum magnitude these forces attain decreases with increasing bottom clearance. These maximum values are also reached later in the wave cycle.

If the clearance is increased further, a point is eventually reached at which the clearance is large enough so that the choking effect does not occur. At this stage, the velocities are higher through the bottom clearance constriction than over the top of the pipeline during the entire wave cycle. So the associated pressure distribution results in a negative lift force throughout the wave cycle, with maximum downward forces occurring under the crests and troughs of the passing waves. The negative lift diminishes to zero at the points of horizontal flow reversal.

As the bottom clearance is increased further, the downward lift effect is gradually reduced. The phase of the force cycle relative to the-wave cycle remains the same, but the magnitude decreases. Eventually, a point is reached where the bottom clearance no longer acts as a constriction to the wave-induced flow. The flow pattern becomes approximately symmetrical, and the increased velocities of the horizontal flow diverted over the top and bottom of the pipeline, along with the corresponding 


\section{WAVE PROFILE}

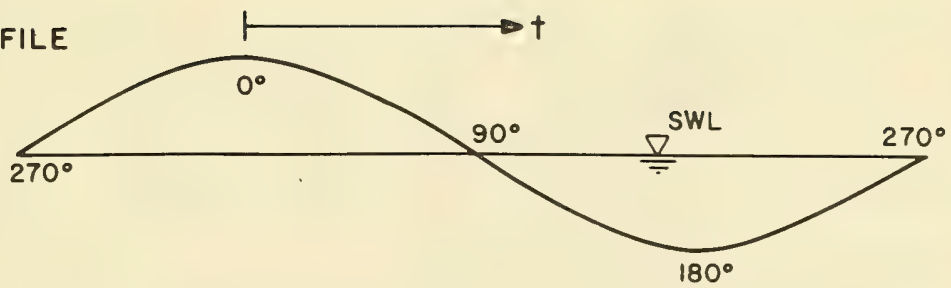

LIFT FORCE

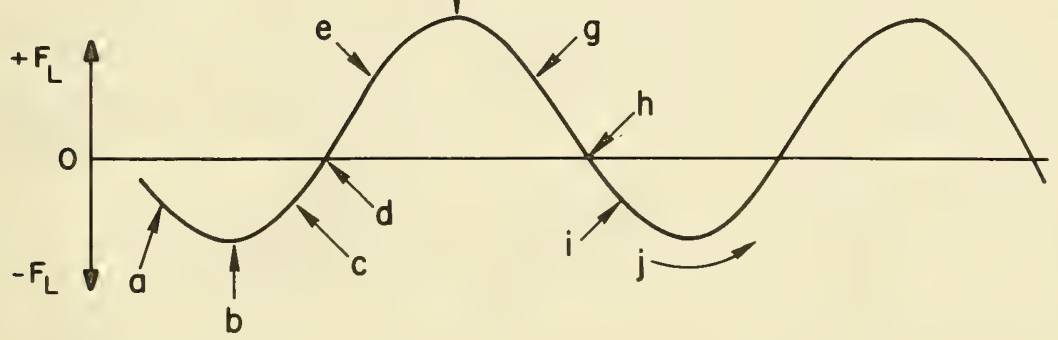

Figure 3. Lift force phenomenon.

(a) Unrestricted flow through the bottom clearance at low velocities results in downward lift force.

(b) Unrestricted flow through the bottom clearance at higher velocities increases the negative iff.

(c) Choking effect begins, so downward lift force decreases.

(d) Velocities increase and pressures decrease at a faster rate over the top of the pipe section than in the restricted flow through the bottom clearance, so the lift force becomes positive.

(e) Upward lift force increases with increasing velocities.

(f) Positive lift reaches a maximum.

(g) Positive lift force decreases and choking effect diminishes with decreasing velocities of the passing wave crest.

(h) Lift force again becomes negative as the flow through the bottom clearance becomes less restricted.

(1) Unrestricted flow through bottom clearance at low velocities results in downward lift force.

(j) Lift force cycle is repeated as the flow reverses with the approaching wave trough. 
pressure distribution, become approximately equal over both sides of the pipe section. At this point, the lift effect is no longer present, and the lift force term may be neglected in calculating the wave-induced forces acting on the pipeline.

The transition in the lift force cycle with increasing bottom clearance is shown in Figure 4.

\section{Mode1 for Wave-Induced Lift Forces.}

The traditional lift force equation, derived for unidirectional steady-flow situations, is expressed as $F_{L}=1 / 2 C_{L} \rho A u^{2}$, where $C_{L}$ is the coefficient of $1 \mathrm{ift}$. This equation has been applied to wave-induced lift forces, using the horizontal component of the oscillating water particle velocity, $u$, in the relationship. The lift force expressed in this way assumes that the force acts in one direction only (either upward or downward) throughout the entire wave cycle.

A pipeline located on the ocean floor with no clearance will experience an upward lift force throughout the entire wave cycle, increasing with the horizontal velocities to reach maximum values under the crests and troughs of the passing waves, and diminishing to zero as the horizontal velocities go to zero at the point of flow reversal. This phenomena is described adequately by the above lift force equation with a positive coefficient of lift $\mathrm{C}_{\mathrm{L}}$.

A pipeline located at a large enough clearance above the bottom so that the choking effect does not occur will experience a downward lift force throughout the wave cycle, since the flow is always faster through the bottom constriction than over the top of the pipeline. Again, this negative lift force increases with the horizontal water particle velocities, reaching maximum magnitudes under the crests and troughs of the passing waves, and decreasing to zero as the flow reverses. This phenomenon is also suitably expressed by the traditional lift force equation, but using a negative coefficient of lift.

These two situations represent the extreme cases bounding the lift force phenomena. However, the choking phenomenon will occur at any clearance between these two limits, and the traditional lift force equation cannot be used to accurately describe the forces exerted on a pipeline. This equation must be replaced by a model developed specifically for wave-induced lift forces. The experimental results of this investigation demonstrate that the largest wave-induced ift forces occur at these intermediate clearances, where the choking phenomenon does develop.

Since the lift force phenomenon is repeated twice per wave cycle with the reversal of the horizontal flow pattern, the lift force can be described mathematically by a sinusoidal function of twice the frequency of the waves. In addition, the mathematical expression must allow for description of the following lift force properties: 
$\begin{array}{lllll}270^{\circ} & 0^{\circ} & 90^{\circ} & 180^{\circ} & 270^{\circ}\end{array}$

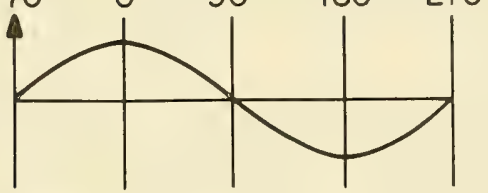

WAVE

PROFILE

(a)

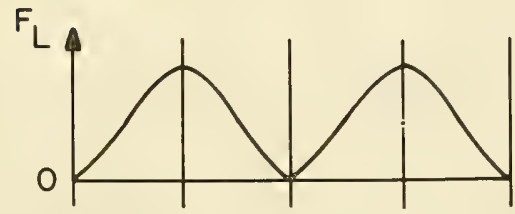

PIPELINE ON BOTTOM

(b)

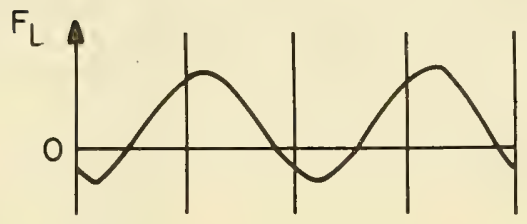

SMALL

CLEARANCE

(c)

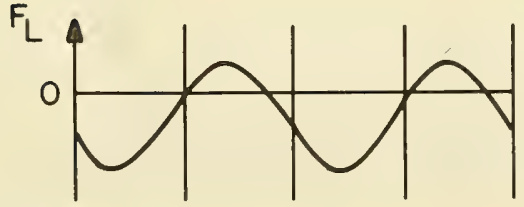

INCREASING

CLEARANCE

(d)

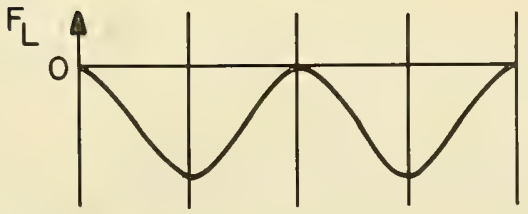

NO CHOKING EFFECT

(e)

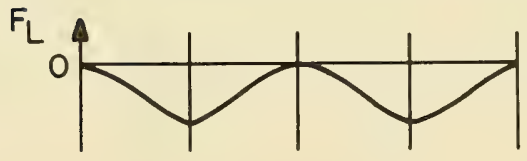

LARGER

CLEARANCE

Figure 4. Change in lift force record for increasing bottom clearance. 
(a) The lift force may be positive during part of the wave cycle and negative for the rest of the cycle. The proportion of positive lift to negative lift may range from all positive lift to all negative lift.

(b) The positions of the maximum values of both the upward and downward lift forces will shift with respect to the position of the wave cycle as the bottom clearance is increased (for a given pipeline and wave condition).

(c) As the clearance is increased, the maximum value of the upward lift force will decrease, while correspondingly the maximum value of the downward lift force will increase.

(d) When the bottom clearance is increased to a point at which the lift effect is downward throughout the entire wave cycle, further increases in clearance will result in decreases in the maximum magnitude of the downward lift force, but without a shift in the position of the maximum lift force with respect to the position of the wave cycle over the pipeline.

A lift force equation of the form,

$$
F_{L}=1 / 2 C_{L} \rho A u_{\max }^{2}\left[\cos ^{2}(\theta-\phi)-k\right] \text {, }
$$

allows an adequate mathematical description of all the above properties of the wave-induced 1 ift force phenomena. This equation fits the experimental data reasonably well over the wide range of conditions tested.

The parameters involved in this modified form of the traditional lift force equation are:

$$
\begin{aligned}
& \mathrm{C}_{\mathrm{L}} \quad=\text { coefficient of lift } \\
& \rho \quad=\text { mass density of fluid } \\
& \text { A } \quad=\text { projected area of pipe section } \\
& u_{\max }=\operatorname{maximum} \text { value of horizontal component of water } \\
& \text { particle velocity at center of pipe section if } \\
& \text { pipeline was absent }
\end{aligned}
$$

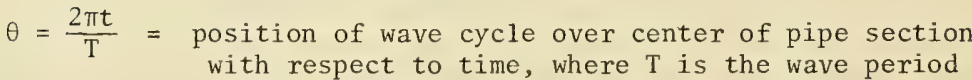

$$
\begin{aligned}
& \text { and } t \text { is the time since the last crest passed over } \\
& \text { the center of the pipe section (see definition } \\
& \text { sketch in Fig. 5). The wave crest corresponds to } \\
& \theta=0^{\circ} \text { ( } 0 \text { radians) or } 2 \pi t / T=0 \text { radians. The wave } \\
& \text { trough corresponds to } 180^{\circ} \text { ( } \pi \text { radians) or } \\
& 2 \pi t / \mathrm{T}=\pi \text { radians }
\end{aligned}
$$




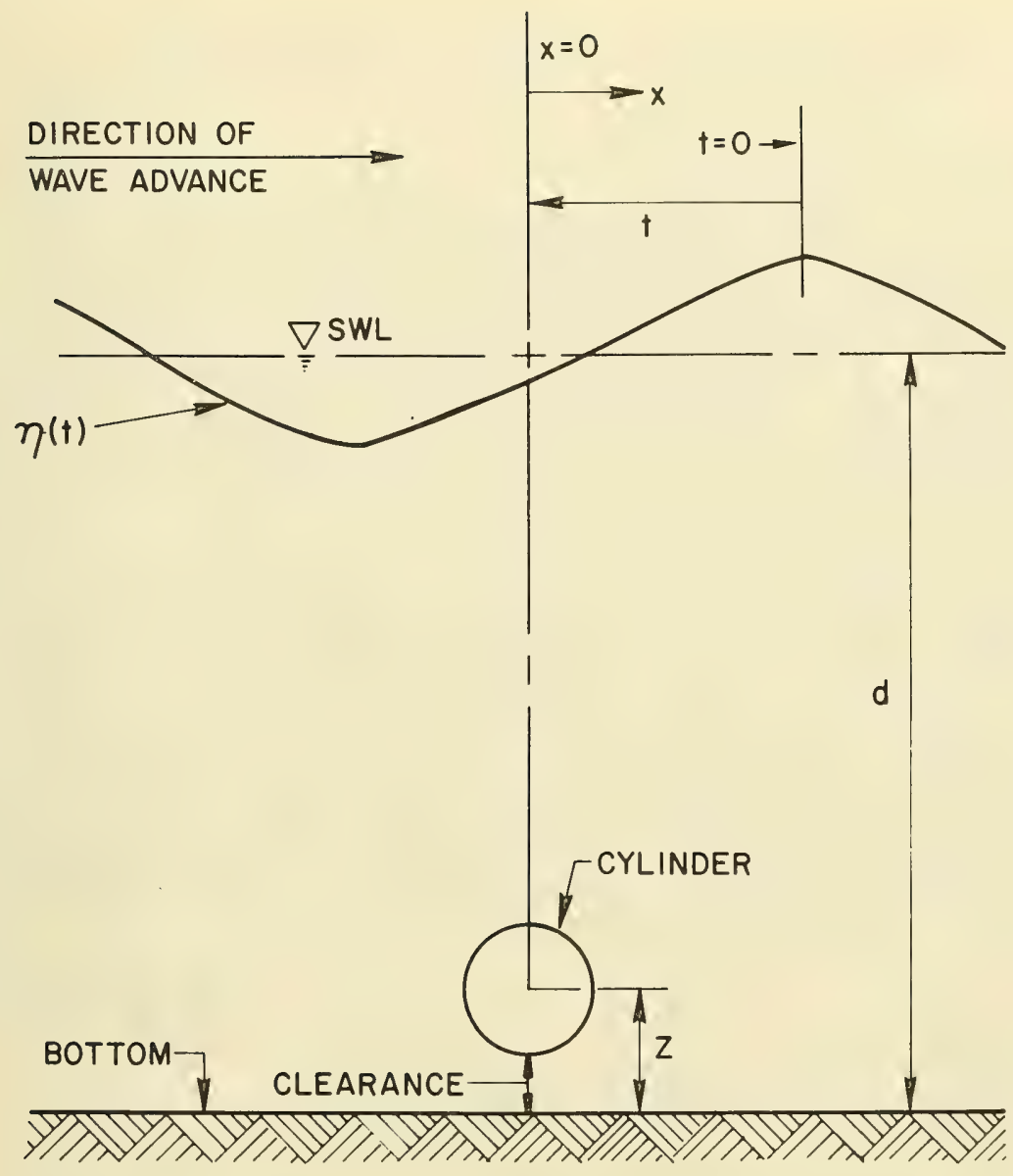

Figure 5. Definition sketch. 
The parameter, $k$, represents the increase in the magnitude and duration of the negative lift forces acting on a pipeline with increasing bottom clearance, and the corresponding decrease in the magnitude and duration of the positive lift forces. The value of $k$ varies from a minimum of 0 to a maximum value of $1 . k=0$ corresponds to the case of a pipeline lying on the bottom with no clearance, in which the lift forces are positive throughout the wave cycle. $k$ increases with increasing bottom clearance to a maximum value of 1 , which corresponds to the case of a pipeline located at a sufficient clearance from the bottom so that the choking phenomenon does not occur, and in which the lift forces are therefore negative throughout the wave cycle.

The phase shift parameter, $\phi$, represents the shift in the position of the maximum values of both the positive and negative lift forces with respect to the wave cycle as the bottom clearance increases. The value of $\phi$ may range from $0^{\circ}$ to a maximum value of $90^{\circ} . \phi=0^{\circ}$ corresponds to the case of a pipeline located on the ocean floor with no bottom clearance, in which the lift forces are positive throughout the wave cycle with maximum forces occurring under the crests and troughs of the passing waves. $\phi$ increases with increasing bottom clearance to a maximum value of $90^{\circ}$, corresponding to a pipeline located above the bottom at a sufficient clearance so that the choking effect does not occur; the lift forces are negative throughout the wave cycle with maximums occurring under the crests and troughs of the waves. As defined, $\phi=0^{\circ}$ when $\mathrm{k}=0$, and $\phi=90^{\circ}$ when $\mathrm{k}=1$, or vice versa.

The coefficient of lift, $C_{L}$, in this form of the lift force equation will always have a positive value, since negative values of the lift force are accounted for by the value of the parameter, $k$. The lift force equation is shown graphically in Figure 6.

To apply the lift force equation to a practical design situation, values of $\mathrm{C}_{\mathrm{L}}, \mathrm{k}$, and $\phi$ must be determined for a given set of pipeline and wave conditions corresponding to the particular case under consideration. Selection of the appropriate values requires quantitative knowledge of the functional relationships between these parameters and the wave conditions, bottom clearance, and pipeline size and configuration. The development of these relationships was the purpose of the experimental part of this investigation.

In a real situation, a pipeline on the ocean floor is often laid over an irregular bottom, supported by the high points in the bottom topography but probably spanning the depressed areas. In this case, the pipeline must be broken into component sections of the same approximate bottom clearance for a separate analysis of each section. The results of the 

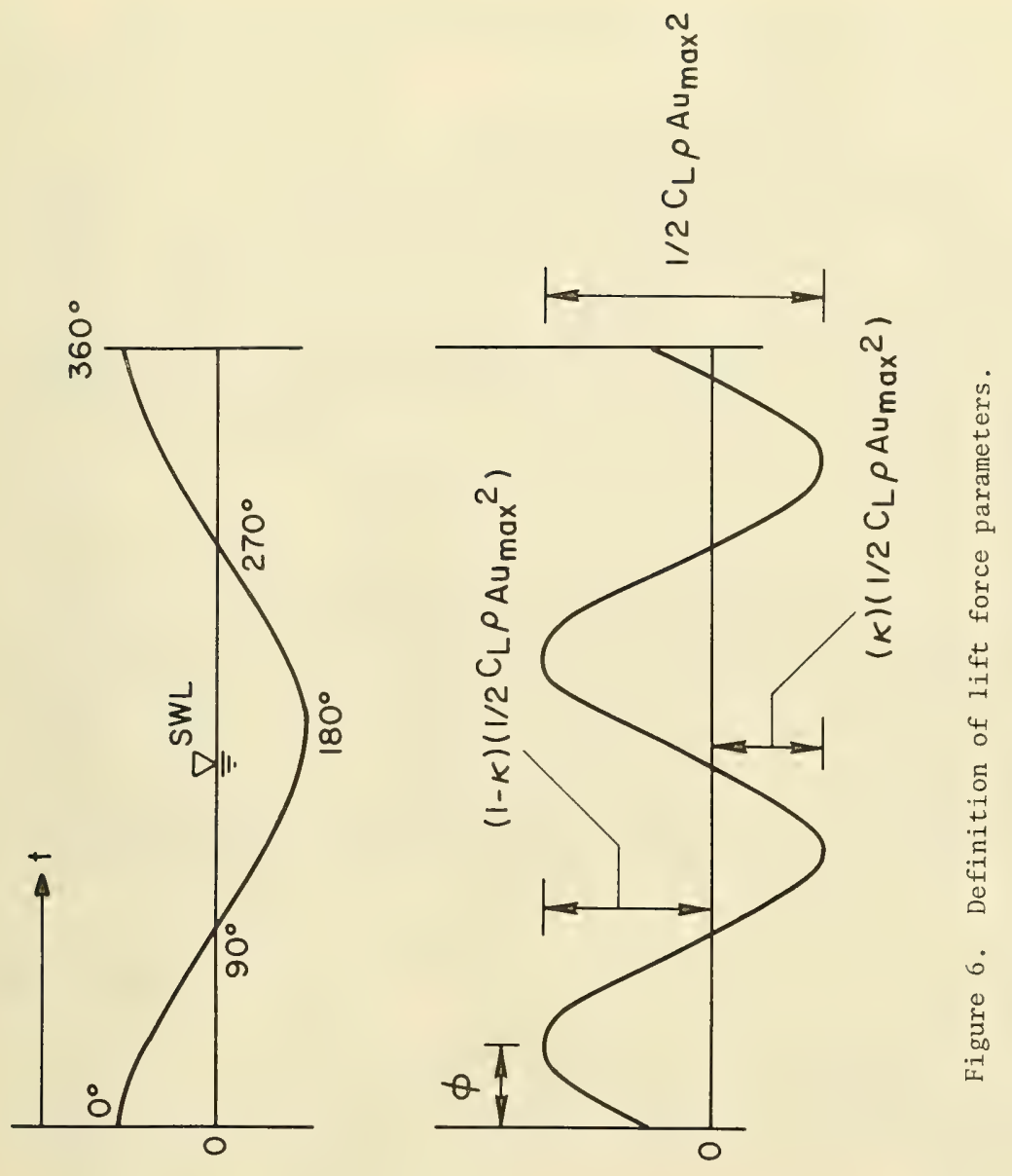

$w$
$w$
$\frac{1}{u}$
3
$\frac{0}{0}$
$a$

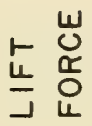


analysis will yield the lift force record (both magnitudes and time history) of each separate component pipe section, which may then be integrated in the appropriate manner to determine the maximum waveinduced stresses exerted on the pipeline at any critical section.

This is important because the maximum lift forces may act upward on a bottom-supported section of a pipeline, while acting downward on the adjacent sections of the pipeline spanning the bottom at a small clearance. Maximum values of both the positive and negative lift forces acting in opposite directions could easily occur at the same point in the wave cycle (under the crests and troughs), thus exerting stresses on the pipeline twice as high as would be calculated considering any pipe section alone, or in using some average clearance for a long section of the pipeline.

4. Extension of Model to Higher Order Theories.

The lift force model (eq. 4) is based on linear theory, assuming the lift force phenomenon is identical as either the wave crest or trough passes over the pipeline. Such a symmetrical expression is not flexible enough to consider slightly different kinematics under the wave crests and troughs, which are expressed in higher order theories. These different kinematics would, in reality, produce slightly different lift forces under the crests and troughs of nonlinear waves.

The lift force model described above was derived as a modification of the traditional lift force equation using linear wave theory to express the horizontal water particle velocities. Using linear wave theory, the traditional lift force equation can be expressed as:

$$
\mathrm{F}_{\mathrm{L}}=1 / 2 \mathrm{C}_{\mathrm{L}} \rho \mathrm{A} u_{\max }{ }^{2} \cos ^{2}(\theta)
$$

This equation was modified to make it a suitable expression for waveinduced lift forces by adding the phase shift parameter, $\phi$, to account for maximum lift forces occurring in places other than the crest andtrough in the wave cycle, and by adding the parameter, $k$, to account for positive lift forces during part of the wave cycle and negative forces during the rest of the cycle. This modified equation fits the experimental data very well for all conditions tested in this investigation.

The model was developed after thorough inspection of the experimental data. For a given pipe diameter and wave condition, the force record followed a sinusoidal relationship of twice the frequency of the waves. As the clearance increased, the maximum positive forces gradually diminished while continuously shifting to a-maximum of $90^{\circ}$ from the wave crest as the forces went to zero (Fig. 4). At the same time, the maximum negative forces slowly grew from a minimum value of zero at a position 
$90^{\circ}$ from the wave crest and increased while continuously shifting positions to reach a maximum negative value at a position $180^{\circ}$ from the wave crest (Fig. 7, a).

Since a sinusoidal function of twice the frequency of the wave $\left(\sin 2 \theta\right.$ or $\cos 2 \theta$ ) can be expressed as $\cos ^{2} \theta$, using the appropriate trigonometric relationships, and since the lift force is a function of the horizontal velocity squared $\left(u_{\max } \cos \theta\right)^{2}$, using linear wave theory, the lift force equation was expressed as $F_{L}=1 / 2 C_{L} \rho A u_{\max }{ }^{2}\left[\cos ^{2}(\theta-\phi)-k\right]$.

However, it is the symmetrical properties of this equation and linear wave theory that allow this expression to work so well. When higher order wave theories are applied to this relationship, problems due to nonsymmetry are encountered. This is easily seen by graphically comparing the transition from positive to negative lift forces with increasing bottom clearance with this lift model, using both linear and higher order theories.

The horizontal component of the water particle velocity for both Stokes' third-order waves and linear waves is shown in Figure 8, along with the corresponding lift forces on a pipeline for the two extreme cases of: (a) a pipeline on the bottom with no clearance, and (b) a pipeline with a large enough bottom clearance so that the choking phenomenon does not occur. By gradually shifting the linear theory lift force curve for case (a) (no bottom clearance) to the right $90^{\circ}$ from the wave crest, while simultaneously lowering it so that the forces become negative, the lift force curve for case (b) is obtained (compare Figs. 7 and 8 ). This same transformation of the wave force record was observed with increasing bottom clearance in the experimental data.

However, if this procedure is repeated with the Stokes third-order lift force record, the correct force record for case (b) is not obtained (compare Figs. 7 and 8 ). In reality, rather than a mere shift of the force record downward and to the right with increasing bottom clearance, a simultaneous transformation of the shape of the lift force record would also occur for highly nonlinear waves. This gradual transformation of the shape occurring simultaneously with the shift would provide a continuous change in the lift force record with increasing clearance between the two limiting cases (a) and (b) (Fig. 8).

However, the lift force phenomenon is not a direct function of the instantaneous water particle velocity acting at the center of the pipe section if the pipeline was absent. Rather, it is a complicated function of the asymmetrical distorted flow pattern and accelerating velocity field acting on the pipeline, which in turn causes the choking phenomenon to occur, with the resulting change in the relative differences in the flow velocities and corresponding pressure distribution over the top and bottom of the pipeline. Boundary layer flow through the bottom constriction, the formation of a turbulent jet and associated eddies, and a cyclic change in the location of the stagnation point with the accelerating velocity field further complicate matters. In addition, the eddies and 
o. Linear Theory

$0^{\circ} \quad 90^{\circ} \quad 180^{\circ} \quad 270^{\circ} \quad 360^{\circ}$
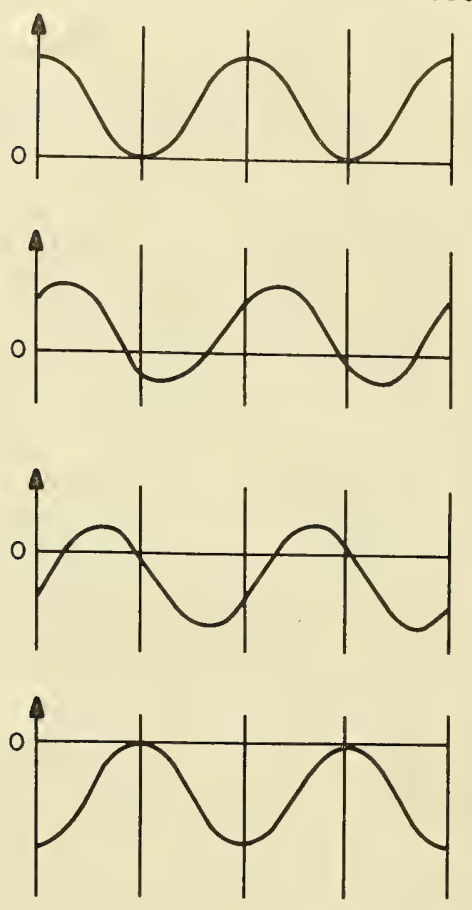

b. Stokes' Third-Order

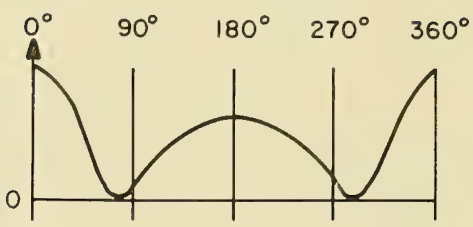

ư

5
0
5
5
0
0
0
2
0
0
$w$
0
0
2
2
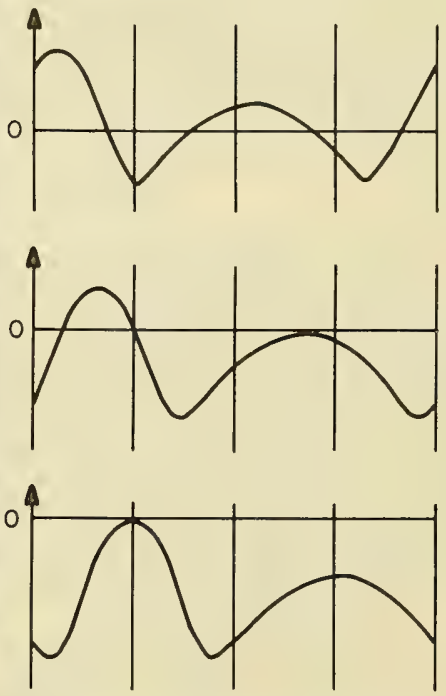

Figure 7. Comparison of linear and Stokes' third-order theories. Simultaneous shift of $1 \mathrm{ift}$ force record as $\phi$ increases from $0^{\circ}$ to $90^{\circ}$ and $K$ increases from 0 to 1 with increasing bottom clearance. 

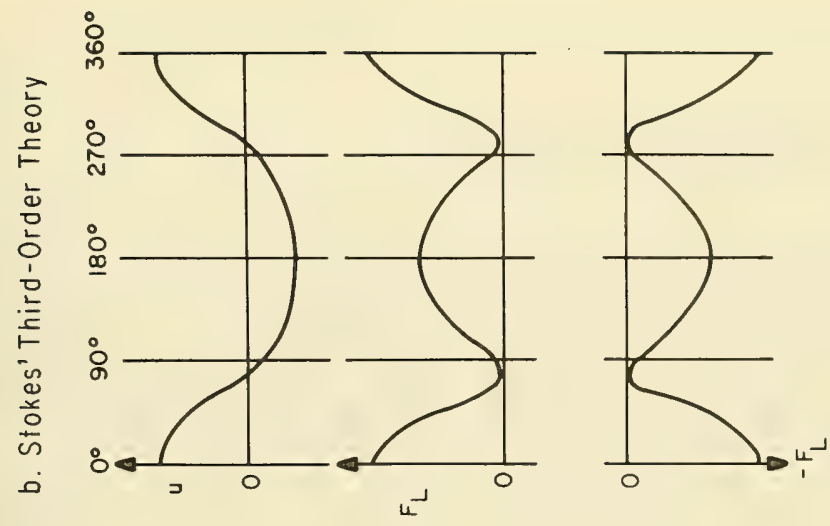

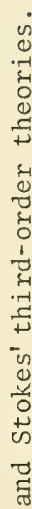

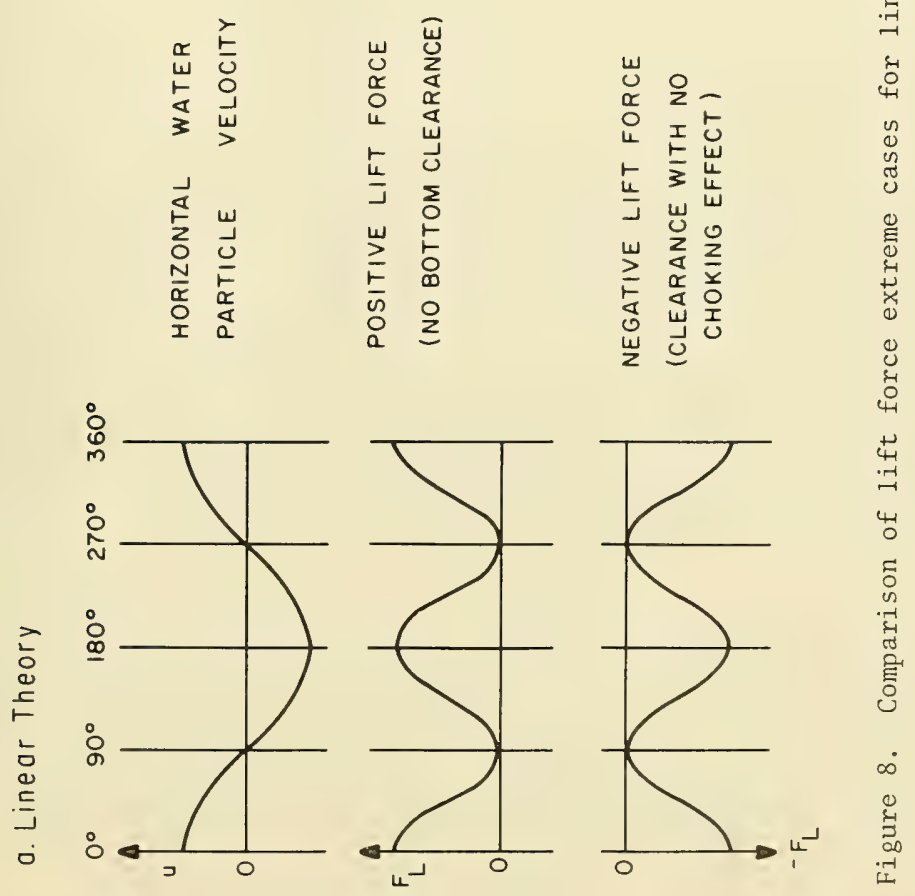


increased turbulence generated by the jet may be swept back through the bottom constriction as the flow pattern reverses with the passing waves.

Because of this, development of an accurate mathematical description of the lift force phenomena for nonlinear waves that would cover the complete transformation of the lift force record with increasing bottom clearance, and yet be flexible enough to allow application of any higher order theory, would be a formidable, if not impossible, task. Since the lift force model developed for linear theory seems to fit the experimental data reasonably wel1, even for waves that were obviously nonlinear, it should provide a useful tool for engineering calculations, even though it may not be flexible enough and theoretically correct to allow the use of higher order wave theories. The value of the maximum horizontal velocity, $u_{\max }$, can be calculated under the wave crest using any higher order wave theory; this value can then be used in the linear 1 ift force model, possibly giving a better approximation of the 1 ift forces induced by highly nonlinear waves.

\section{I . EXPERIMENTAL INVESTIGATION}

\section{Experimental Equipment.}

Model experiments were performed in three different wave tanks. The two-dimensional tests were done in a 1 -foot-wide wave channel in the Hydraulic Engineering Laboratory. (HEL) at the University of California, Berkeley. The three-dimensional tests were started in the 8-foot-wide Naval Architecture (NA) tow tank, and then continued in the 8-foot-wide HEL wave tank where the majority of the experiments were conducted, both located at the Richmond Field Station of the University of California. The 1 -foot wave channel is 100 feet ( 30.48 meters) 1 ong; the 8 -foot HEL wave tank and NA tow tank are 180 and 200 feet (54.86 and 60.96 meters) long, respectively. All tests were conducted at approximately the middle of the tanks. A stillwater depth of 2 feet ( 60.96 centimeters) was used in the two dimensional tests, and a 3 -foot (91.44 centimeters) water depth was used in the three-dimensional experiments.

A flapper-type generator is located at one end of each of the HEL wave tanks; the NA tow tank has a piston-type wave generator. The wave period is controlled by varying the speed of the electric motors which drive the wave generators. A cam mechanism with a variable stroke length is connected between the drive motor and the flapper, and the wave height is varied by changing the stroke length. A wave filter, consisting of a series of vertical screens, was placed in front of the wave generator in the 1 -foot-wide wave channel to smooth out any irregularities in the generated waves due to reflections from the flapper. A permeable beach was installed at the opposite end of each of the tanks to absorb the wave energy and minimize the wave reflections from that end of the wave tank. 
The wave-induced forces on the model pipe section were measured by a wave force meter designed and built by Al-Kazily (1972). A few modifications were made to make the instrument more suitable for this investigation. The same transducer unit was used in all of the experiments, but fittings of different sizes were made to accommodate test cylinders of various diameters.

The force transducer consists of a strain bar mounted between two supports. The model pipe section is mounted to the strain bar in such a way that forces on the pipe induce bending stresses on the strain bar. These forces are measured by four strain gages mounted to the strain bar at sections of maximum strain, with two gages in compression and the other two in tension. The strain gages are wired in a wheatstone bridge, which is connected to a carrier amplifier which amplifies the output from the strain gages. The signal is then recorded on a strip-chart recorder.

The original strain gages were Bean-type BAB-13-125DD-120S, and were mounted to the steel strain bar with EPY-150 two-part epox', and then coated with Dow Corning Silastic RTV silicon rubber for laterproofing. Shortly after the beginning of the three-dimensional tests, problems were encountered in the operation of the transducer. These problems were caused by the deterioration of the original strain gage adhesive and coating, so new strain gages were installed on the transducer unit. The new gages were Micromeasurement-type EA-06-125AD-120, bonded to the strain bar with Micromeasurement M-Bond 610 two-part strain gage adhesive, and then coated with Micromeasurement M-Coat D and M-Coat G for waterproofing protection. About halfway through the three-dimensional tests, further problems were encountered in the operation of the transducer unit, probably due to water leakage into the waterproof coating. There was also evidence of corrosion on the steel strain bar, so it was decided to build a new force transducer using a stainless-steel strain bar to minimize corrosion, and encapsulated strain gages to minimize problems with water leakage. The new strain gages were Micromeasurement-type CEA-06-125UW-120. The same strain gage adhesive and waterproof coatings were used, with Micromeasurement M-Coat B along the lead wires to minimize the change of water "wicking" along the lead wires to the inside of the coating materials.

The transducer mounting arrangement was different for the twodimensional and three-dimensional experiments. The test cylinder and transducer unit for the two-dimensional tests were mounted between two support brackets on each side of the 1-foot wave channe1. For the three-dimensional experiments, the test cylinder and transducer unit were mounted between two long dummy pipe sections, which were in turn mounted to a steel base. The force meter and mounting arrangement is shown in Figures 9 and 10 for the two-dimensional tests, and in Figures 11 to 15 for the three-dimensional tests.

A paralle:-wire resistance-type wave gage was used to record the waves passing over the model pipe section. The gage was mounted directly over 


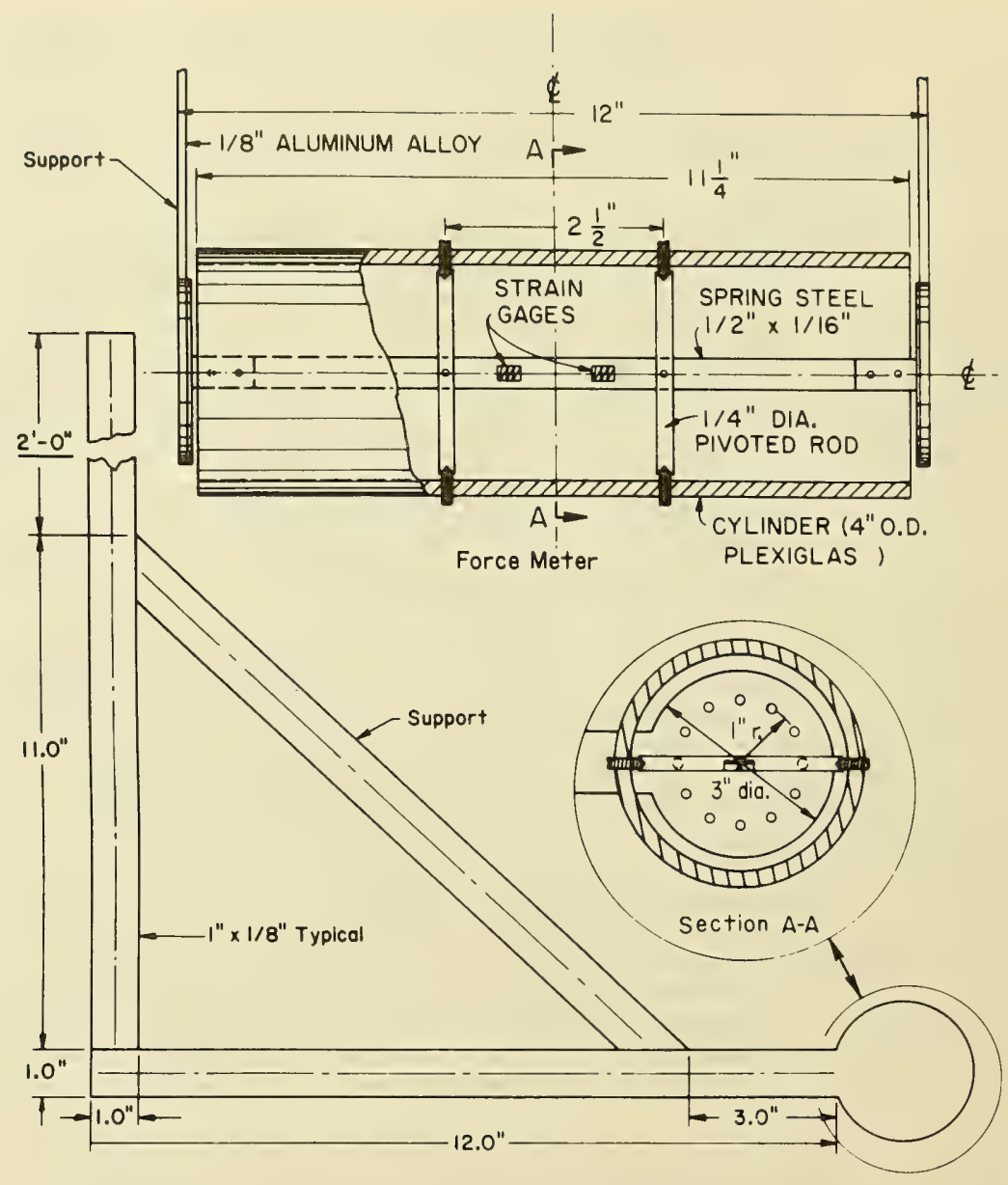

Figure 9. Force meter and support. 


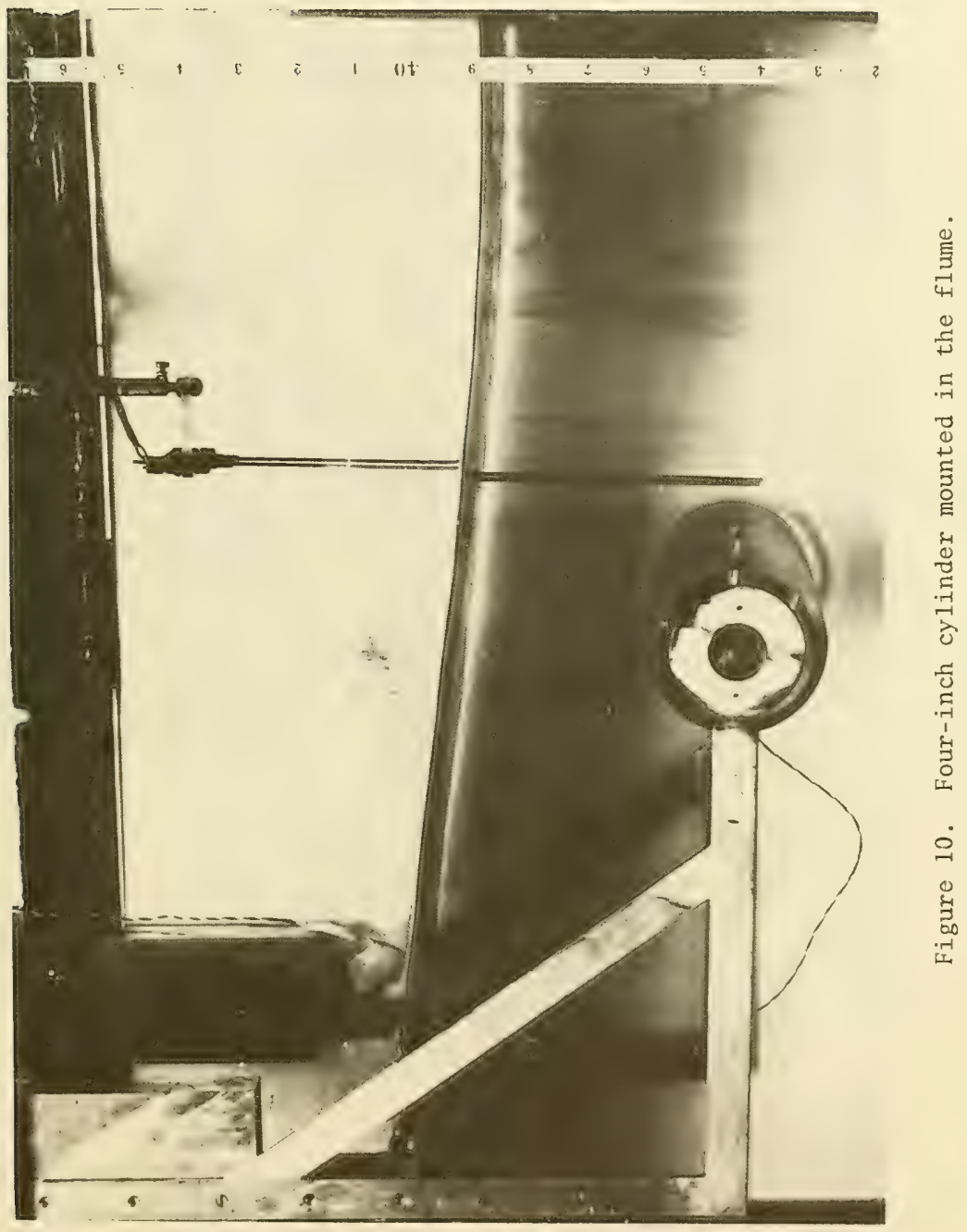




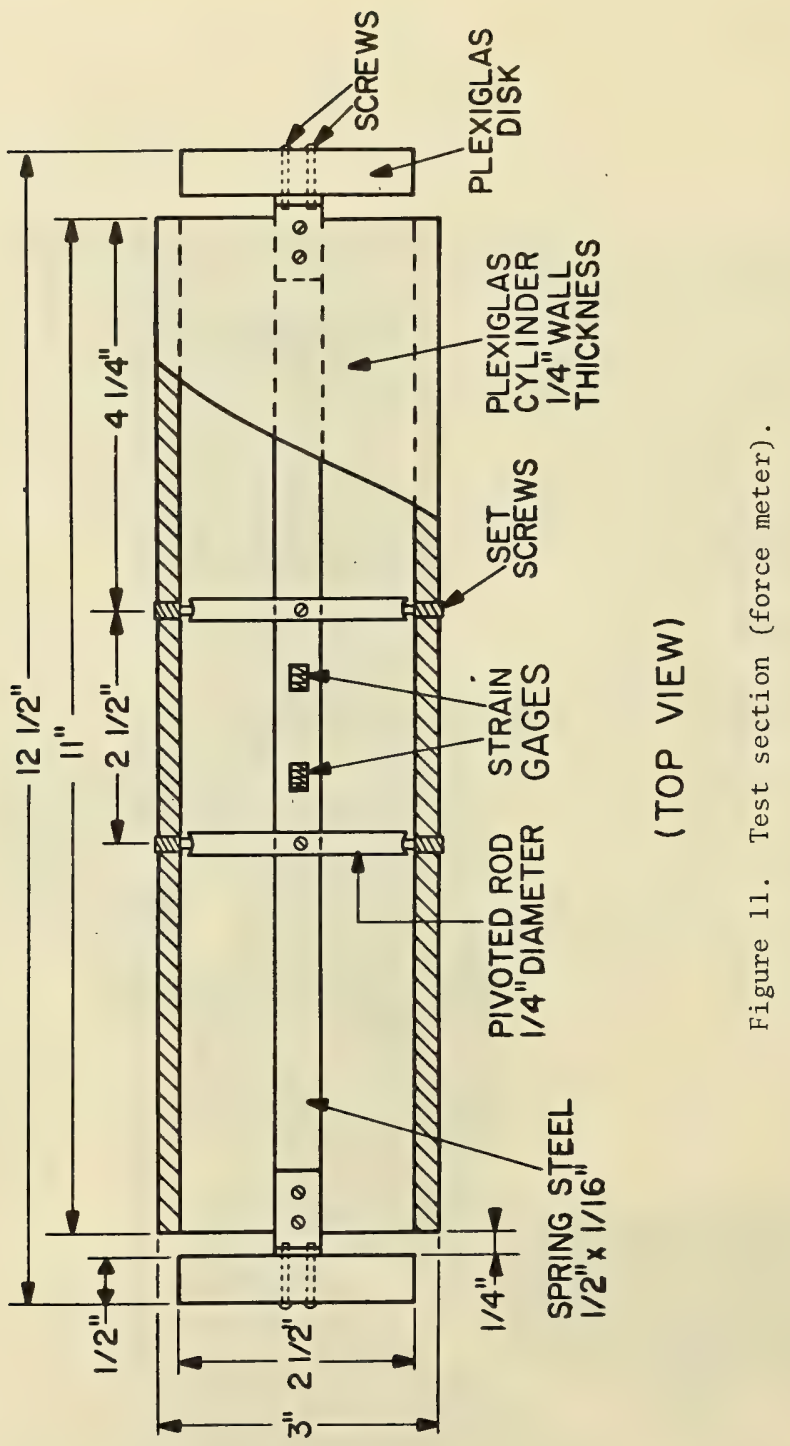




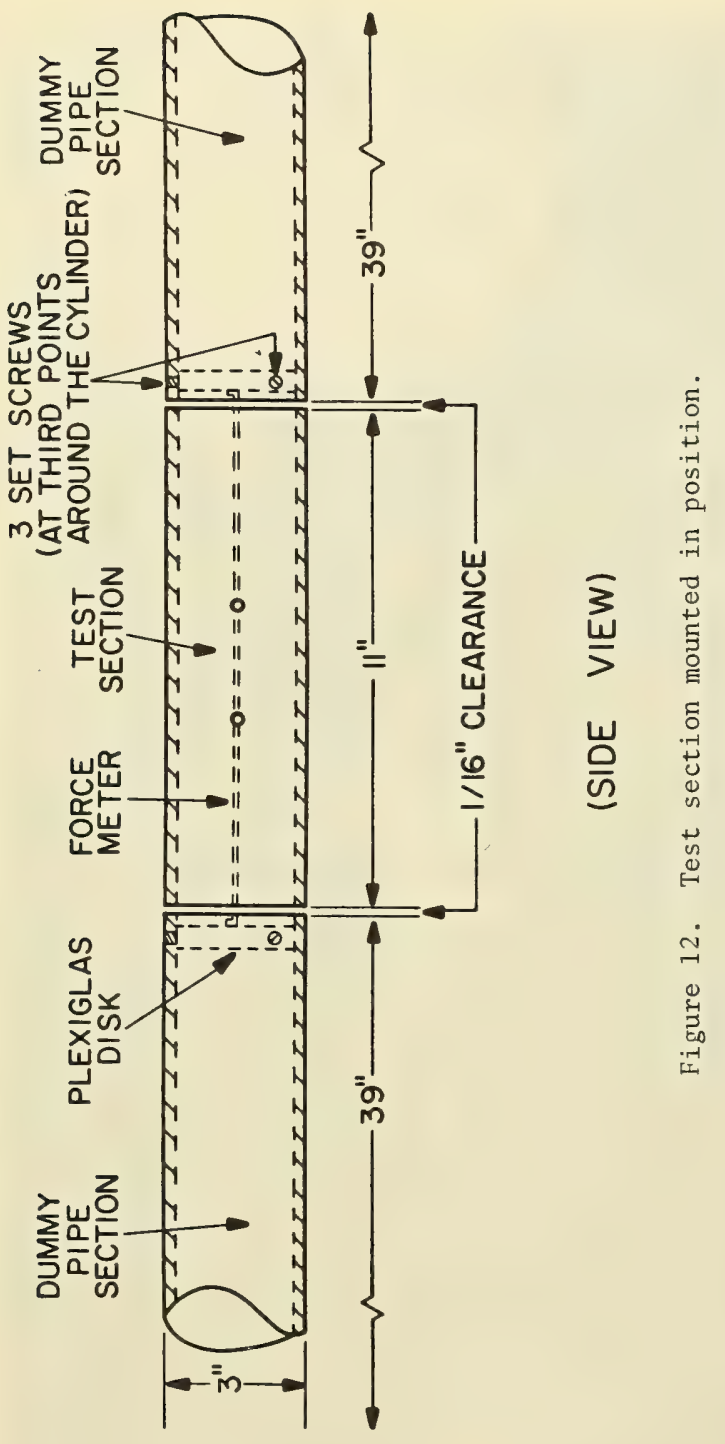




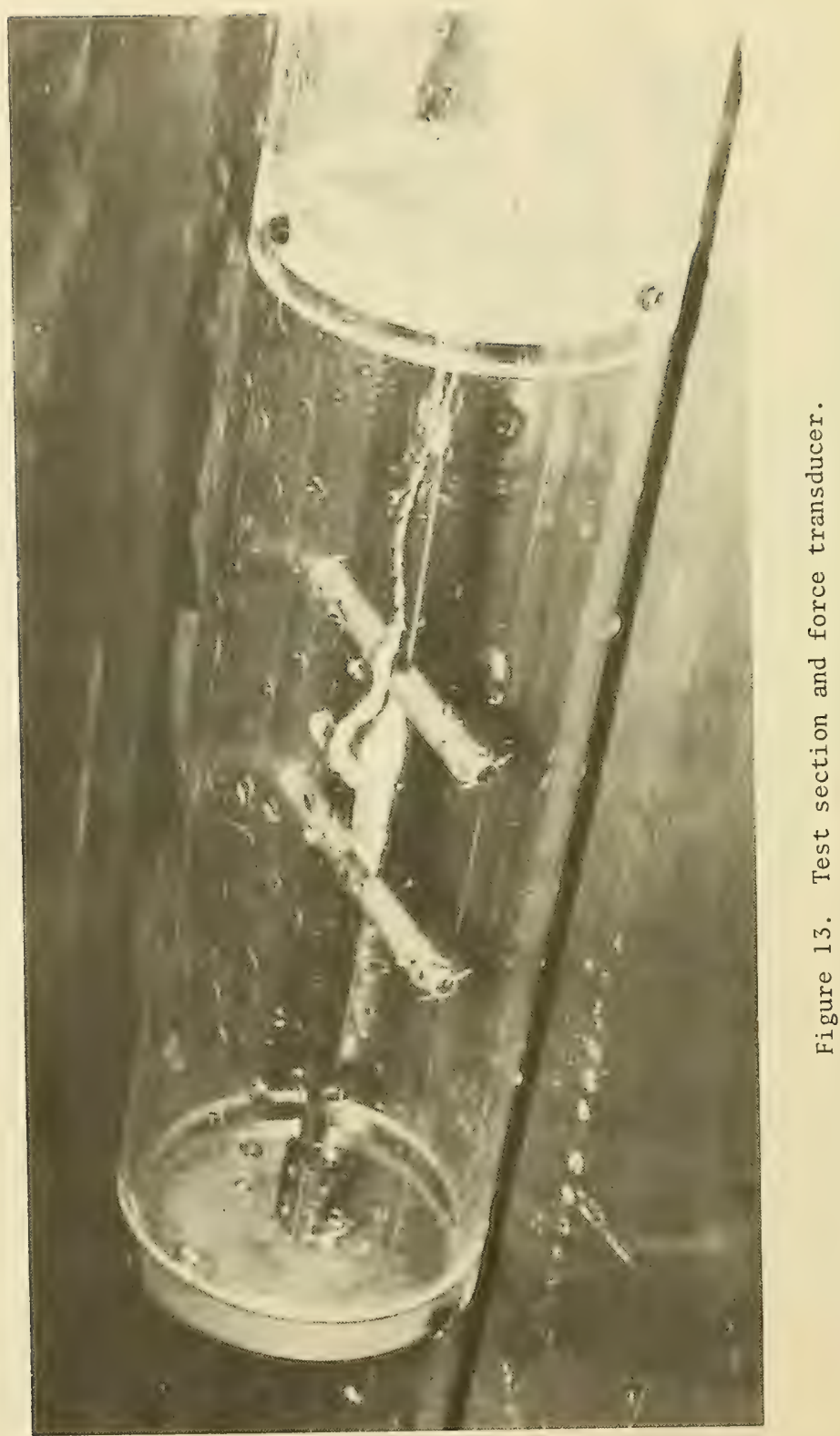




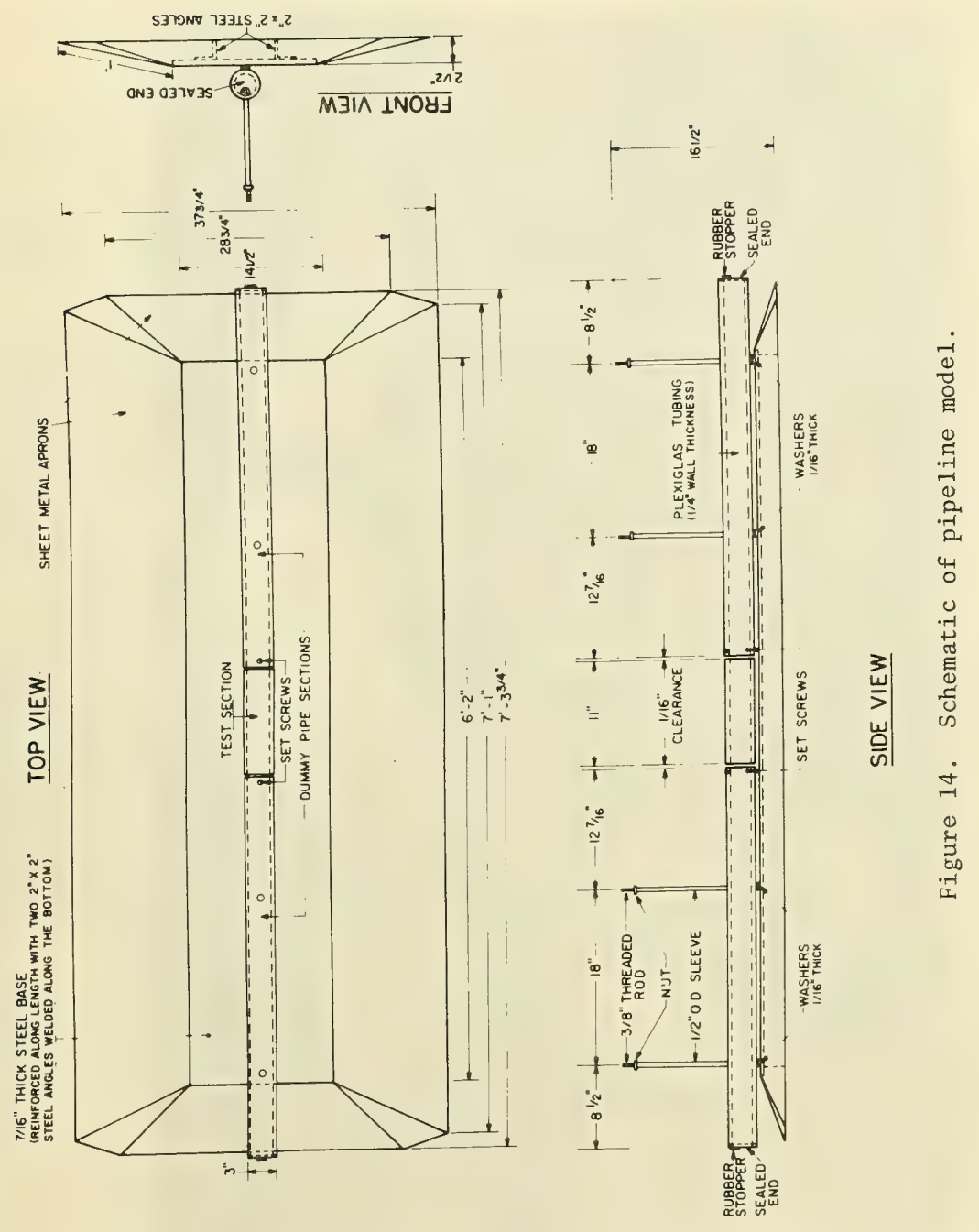




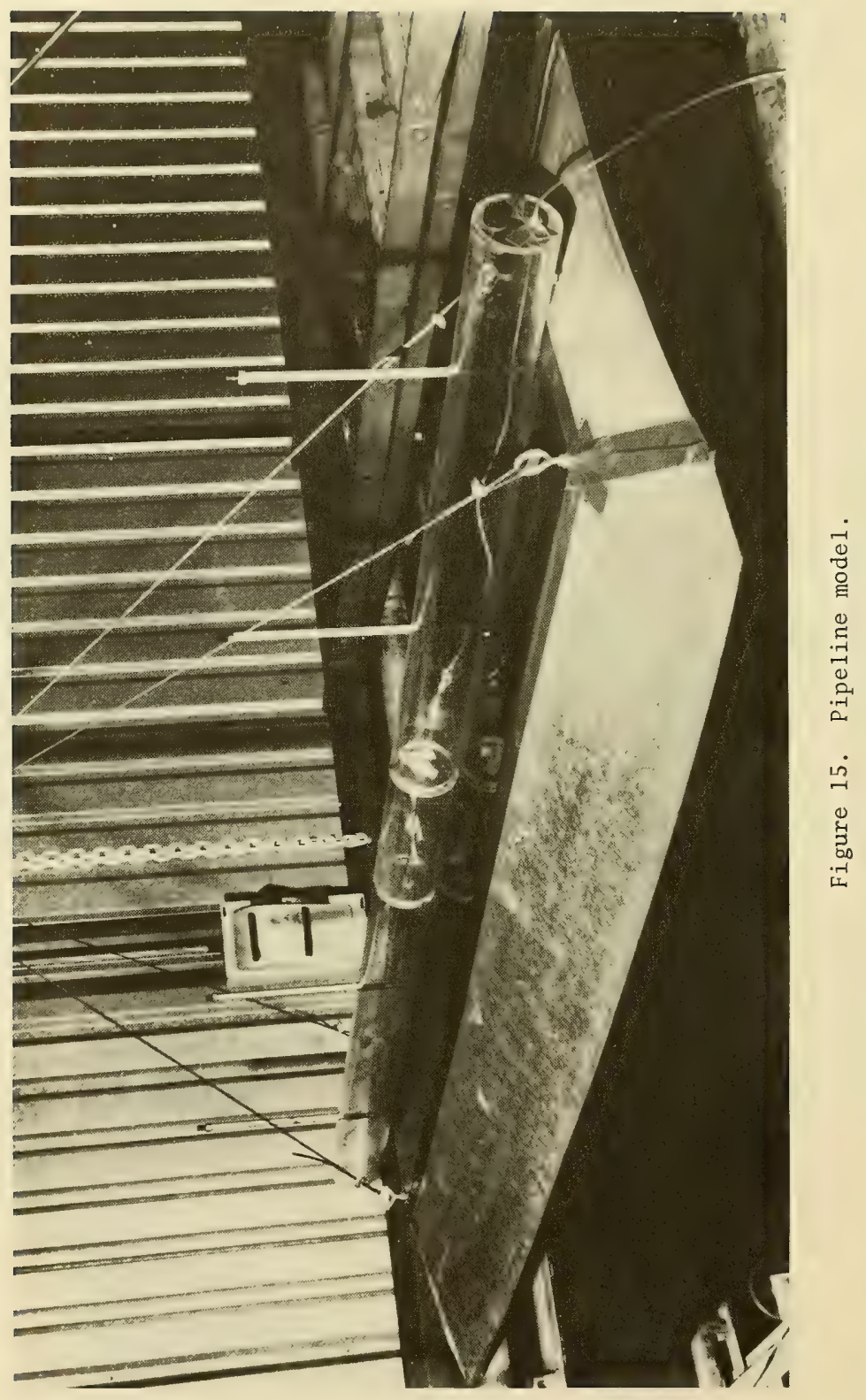


the center of the test section, so that the wave records could be correlated directly with the resulting wave-induced force record.

A Brush dual-strain gage amplifier was used in the experiments, with one channel connected to the wave gage, and the other channel connected to the force meter. The amplifier was connected to a Brush two-channel rectilinear writing recorder which continuously recorded the waves and corresponding wave-induced forces on the pipe section (Fig. 16).

An electronic digital data acquisition system (Paulling and Sibul, 1968) was used in the three-dimensional experiments. The digitizer was connected in parallel with the strain gage amplifier to record simultaneously the wave and corresponding force data on magnetic tape, while at the same time the data were being recorded continuously on the stripchart recorder (Fig. 17). The digitizer sampled alternatingly from both the wave record and force record at a rate of 100 samples per second, resulting in 50 samples per second from each of the two channels.

\section{Procedure for Two-Dimensional Experiments.}

a. Calibration. Both the wave gage and the force transducer were calibrated before each set of experimental runs. The wave gage was calibrated statically by raising and lowering the gage in increments of U.Us root (1.S/ centimeters) and recording the output. The force meter was also calibrated statically by hanging weights in increasing equal increments from a system of pulleys connected to the force meter and recording the output on the strip chart. The force transducer was calibrated in both the upward and downward directions by rearranging the pulley system and repeating the above procedure. The calibration method is shown in Figure 18.

b. Procedure. After calibrating the force meter, the mode1 pipe section was lowered and fixed in a horizontal position at the desired clearance above the bottom of the wave channel, with the long axis of the test cylinder parallel to the approaching wave crests. A sliding point gage was mounted to the wave channel above the pipe section and was used to accurately set the model pipe to the desired bottom clearance and aline the pipe section parallel to the wave crests. Once the mode 1 was in the correct position, the mounting brackets and support struts were clamped to the sides of the wave channe1. The force transducer was mounted in such a way that it was sensitive only to forces acting in the vertical direction.

After the model pipe section was mounted in position, the wave gage was lined up directly over the center of the pipe section with a plumb bob and then clamped in position. The wave gage was then calibrated as described above. The experimental arrangement is shown in Figure 19. 


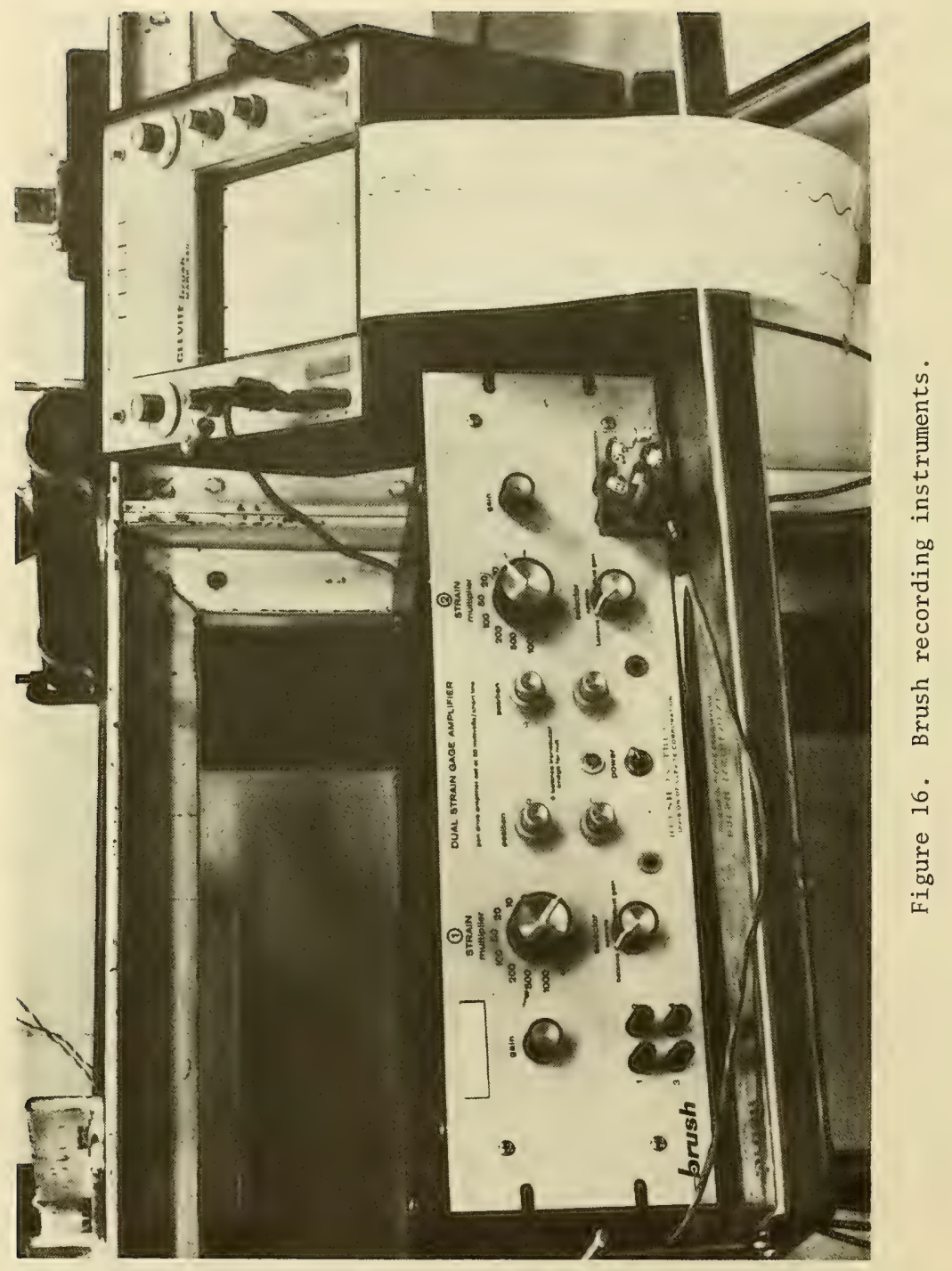




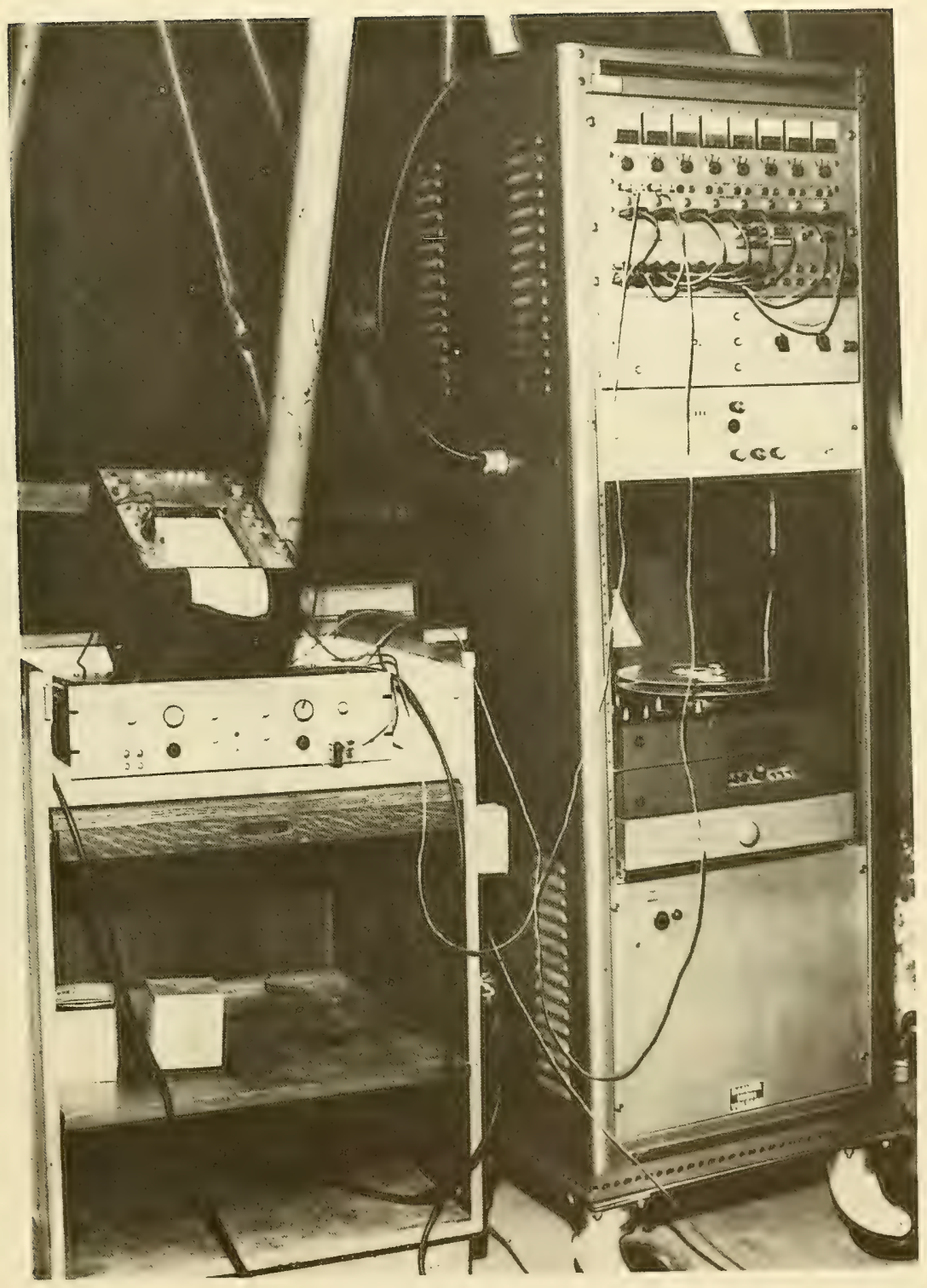

Figure 17. Digitizer and recording instruments. 

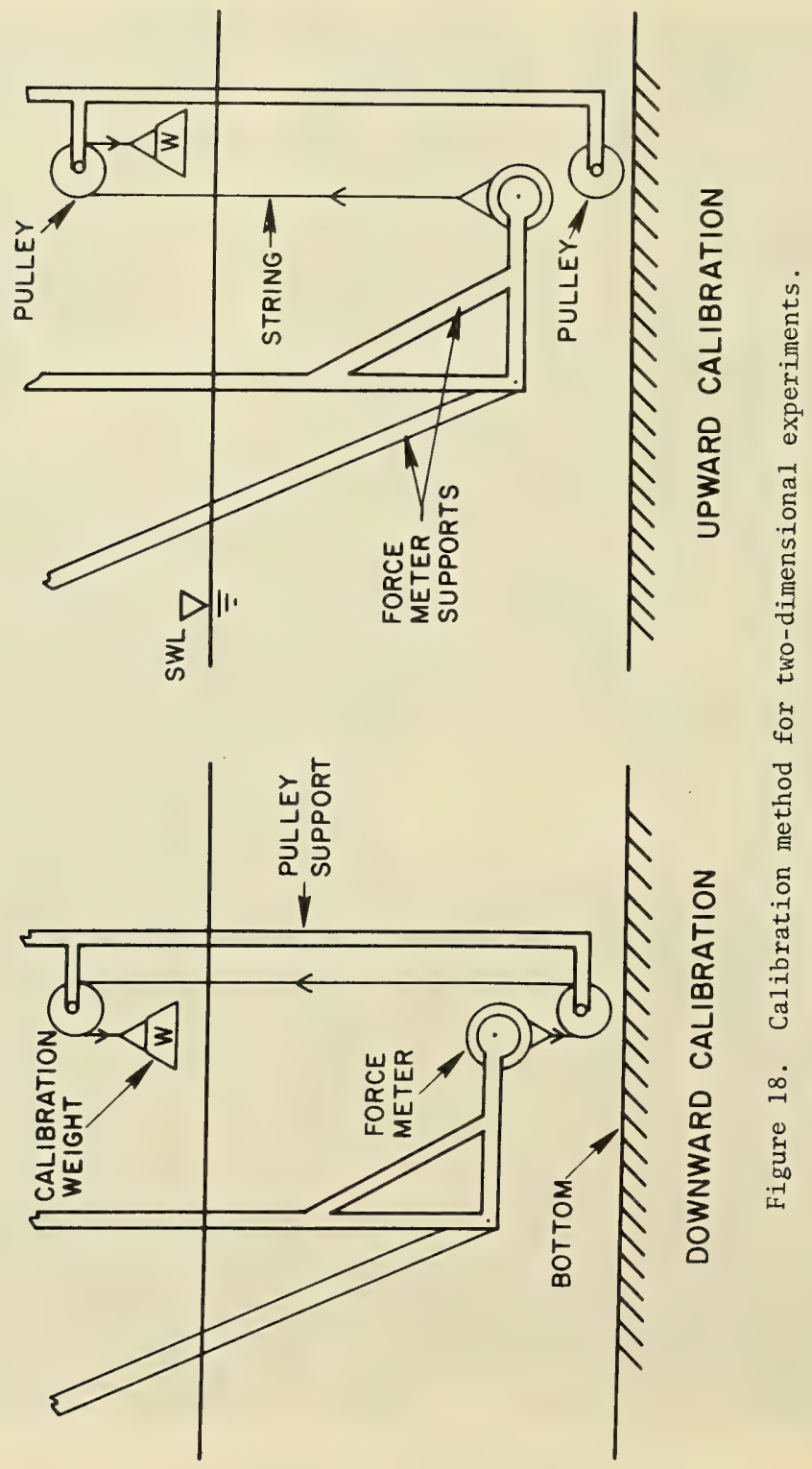


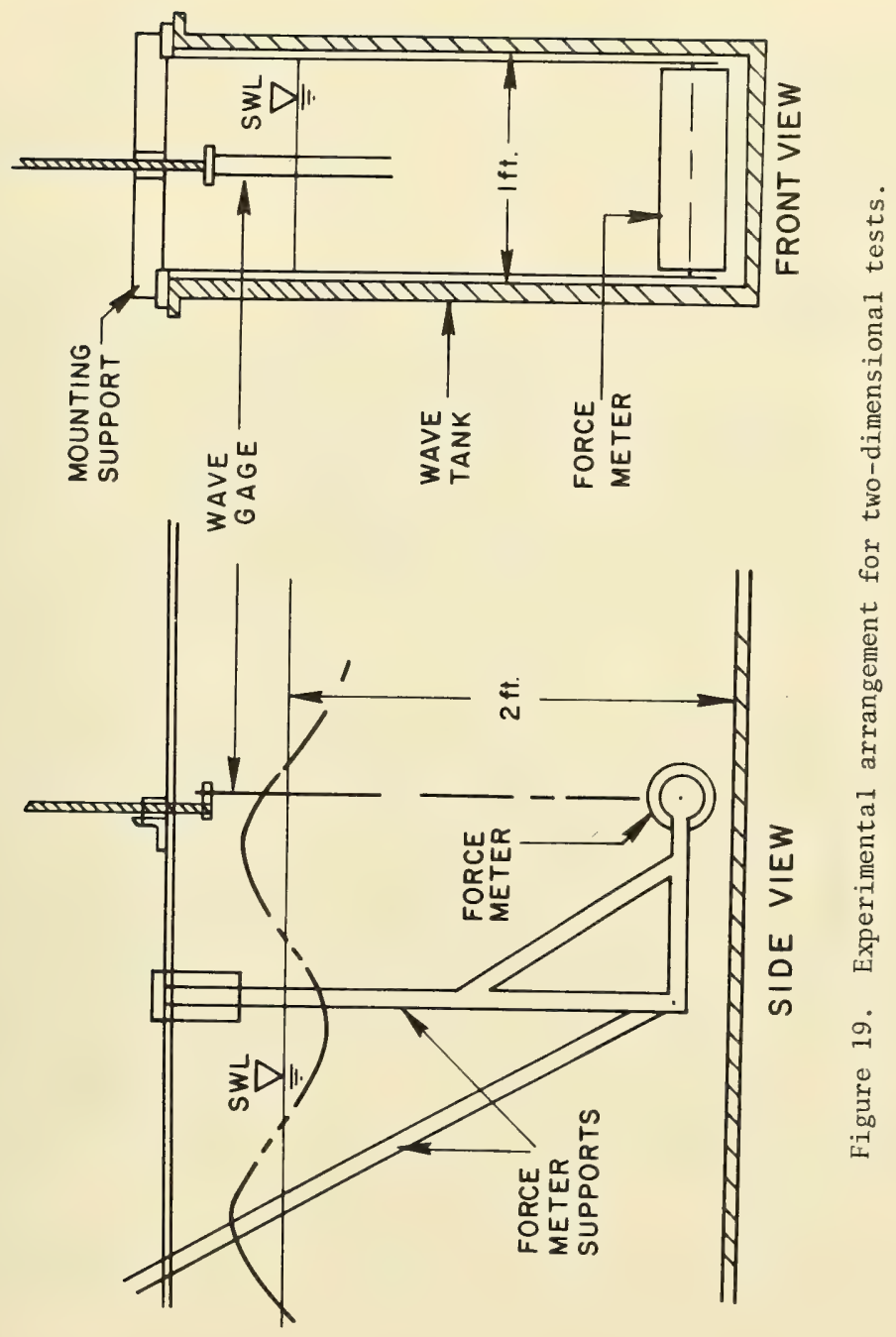


The pipe model and wave gage were mounted in a glass-walled part. of the tank near the middle of the wave channel to facilitate the visual observation of the phenomenon being studied. For each bottom clearance tested, a series of runs was made with waves generated at 19 different wave periods, covering a range of 0.95 to 2.5 seconds. Seven wave heights were generated for each wave period, ranging up to 0.34 foot (10.4 centimeters).

After these runs were completed, the pipeline was set at another bottom clearance, and the procedure was repeated. Seven bottom clearances were tested for each wave condition, ranging from 0.001 foot, $1 / 16,1 / 8,3 / 16,1 / 4,1$, and 2 inches $(0.305,1.59,3.18,4.76$, and 6.35 millimeters, 2.54 and 5.08 centimeters), respectively. The minimum clearance tested $(0.001$ foot) was that which placed the pipe section as close to the bottom as possible without touching the bottom when the waves passed over it. This was necessary to measure any downward forces exerted on the pipe section due to the wave action. The 2 -inch bottom clearance placed the pipe section far enough from the bottom so that the vertical lift forces were insignificant.

These experiments were carried out with a 4-inch-diameter (10.16 centimeters) test cylinder. The experiments were repeated with pipe

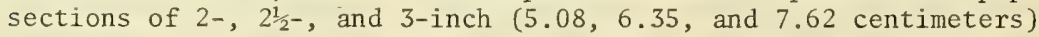
diameters, but only three bottom clearances were tested--0.001 foot, $1 / 8$ inch, and $1 / 4$ inch. The wave conditions covered the same range of wave heights and periods, but were not quite as extensive in number.

In addition to the vertical force measurements, a series of experiments was performed to measure the horizontal forces acting on the pipe section, so that the resultant wave-induced force could be determined throughout the entire wave cycle for several of the experimental conditions tested. Only the 4-inch-diameter test cylinder was used in these experiments, since the corresponding vertical experiments were the most extensive for the 4-inch cylinder. The horizontal forces were measured by rotating the force transducer $90^{\circ}$ so that it was sensitive only to forces acting in the horizontal direction. The calibration procedure was the same as described above for the vertical force measurements except that the system of pulleys was rearranged so that the calibration weights exerted forces in the horizontal direction only.

All seven of the bottom clearances used in the vertical experiments were also used in the horizontal tests. The wave periods covered the same range as the vertical experiments, but only 6 of the 19 wave periods were used-- $0.95,1.25,1.5,1.85,2.25$, and 2.55 seconds. Two of the seven wave heights corresponding to each wave period in the vertical experiments were used in the horizontal tests.

The stillwater depth was held constant at a depth of 2 feet throughout the two-dimensional tests. 


\section{Procedure for Three-Dimensional Experiments.}

a. Calibration. The wave gage and force meter were calibrated before each set of experimental runs. The wave gage was calibrated in the same manner as the two-dimensional tests, but 0.1 -foot $(3.05$ centimeters) increments were used rather than 0.05 -foot increments, since larger waves were used in these experiments.

The force transducer was calibrated in the upward direction in the same manner as the two-dimensional tests, by hanging weights over a pulley to a string attached to the pipe test section. However, because the three-dimensional model was mounted to a base with a small bottom clearance, it was impossible to calibrate the transducer in the downward direction by using a system of pulleys, since there was no room for a pulley between the pipe section and the base to which it was mounted. Rather, the force meter was calibrated in the downward direction by placing the weights directly on top of the center of the submerged test section and using the submerged weight of the weights in calculating the calibration curve. Weight increments of 50 grams were used in calibrating the transducer. The calibration method is shown in Figure 20 .

b. Procedure. An overhead crane was used to lower the pipeline model and base into the wave tank. The assembly was first submerged to a depth of about $1 \frac{1}{2}$ feet ( 45.7 centimeters). The mode 1 was tilted at both ends to remove all air bubbles from the system, and the ends of the dummy pipe sections were stoppered to prevent waterflow through the pipeline mode1. The bottom clearance between the base and the pipe model was adjusted by placing spacers on the support rods between the base and the dummy pipe sections, and then tightening the nuts on the support rods above the dummy pipe sections. The test section was tinen centered and adjusted carefully to the exact bottom clearance desired with the aid of 10 adjusting screws. The calibration string was attached to the test section, and the assembly was lowered to the bottom of the tank.

The calibration string and pulley system was alined directly over the center of the test section with a plumb bob, and the pulley supports were then clamped to the sides of the wave tank. The transducer was first calibrated in the upward direction, after which the calibration string was removed, and the transducer was calibrated in the downward direction, as described above.

The pipeline model was positioned at the desired angle of orientation on the tank bottom by lining up one of the long edges of the model base parallel to the correct line marked on the bottom of the wave tank. Lines were marked on the tank bottom in $15^{\circ}$ increments from $0^{\circ}$ to $75^{\circ}$, where $0^{\circ}$ corresponds to a pipeline parallel to the approaching wave crests. After the model was calibrated and placed in position, the wave gage was lined up directly over the center of the test section with 


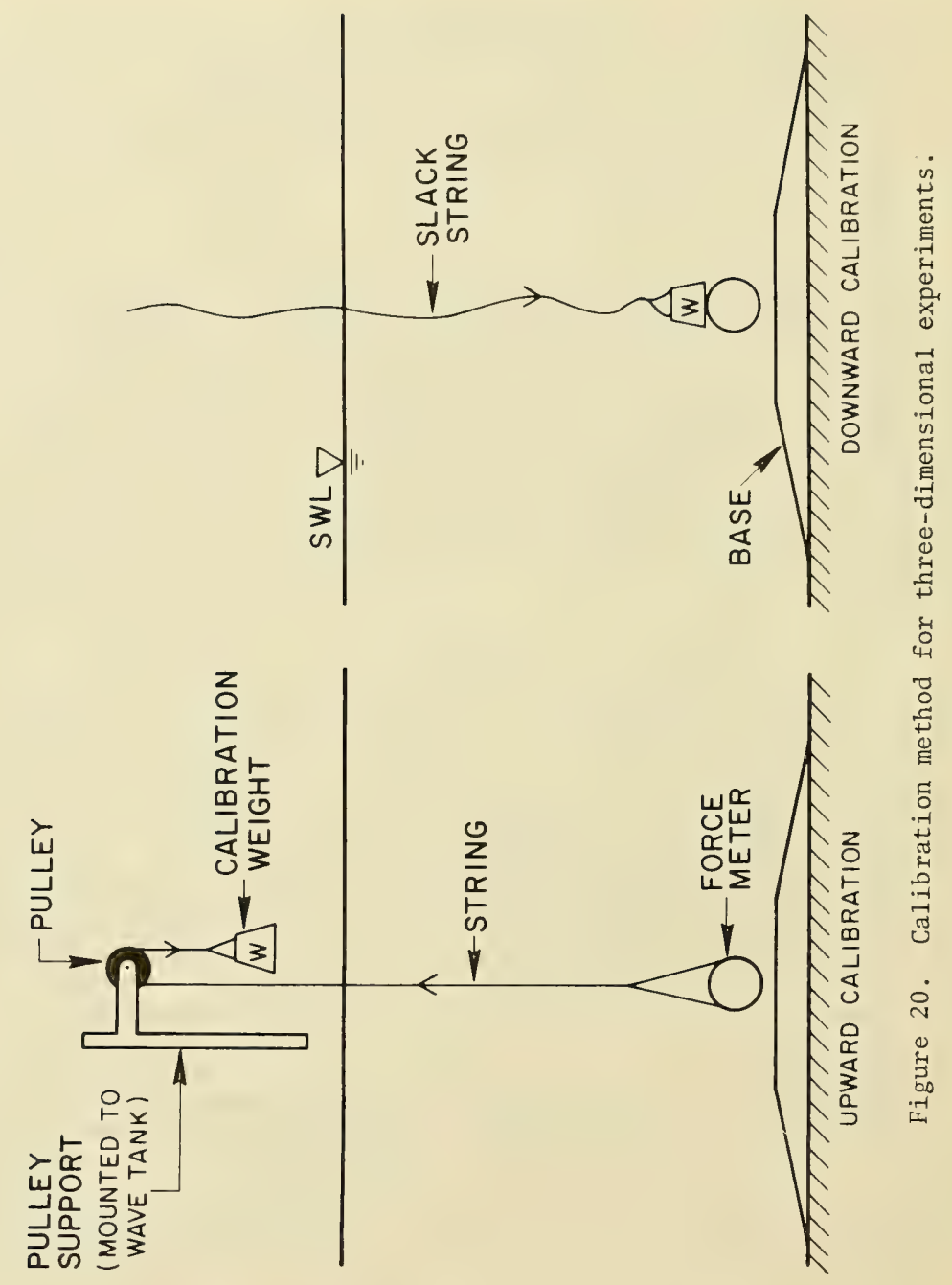


a plumb bob, clamped in position, and then calibrated as described above. The experimental arrangement is shown in Figure 21.

For each bottom clearance, six angles of orientation $\left(0^{\circ}, 15^{\circ}, 30^{\circ}\right.$, $45^{\circ}, 60^{\circ}$, and $75^{\circ}$ ) were tested. Fifteen runs with different wave conditions were made for each bottom clearance and orientation angle. These runs covered four wave periods ranging from 1.4 to 2.6 seconds, with waves generated at four heights for each period, ranging to a maximum of about 0.7 foot (21.3 centimeters). Eight bottom clearances were tested, ranging from 0.001 foot, $1 / 16$ inch, $1 / 8$ inch, 3/16 inch, 1/4 inch, $1 / 2$ inch, 1 inch, and 2 inches.

The above experiments were done using a 3-inch-diameter pipeline mode1. The tests were then repeated using a 2- and 4-inch-diameter pipeline. The 1 - and 2-inch clearances were not tested because the 1 ift forces at these clearances proved insignificant in the previous tests. Also, the tests at an orientation angle of $75^{\circ}$ were eliminated, since the previous experiments demonstrated that the vertical forces measured at this angle were insignificant, and too small to be measured with any accuracy. Aside from these changes, the 4-inch-diameter pipeline was tested at the same bottom clearances, orientation angles, and wave conditions as the 3-inch-diameter model. The 2-inch-diameter model was tested at the same bottom clearances and wave conditions, but only three of the five orientation angles $\left(0^{\circ}, 30^{\circ}\right.$, and $\left.60^{\circ}\right)$ were tested.

The stillwater depth in the wave tank was held constant at 3 feet throughout the three-dimensional experiments, but since the base of the pipeline model was located $2-7 / 16$ inches (6.19 centimeters) above the tank bottom, the effective stillwater depth over the pipeline base was 2.797 feet ( 85.25 centimeters). The definition sketch for the threedimensional experiments is shown in Figure 22.

\section{Data Reduction.}

The wave force data were taken on a two-channel strip-chart recorder with the paper advancing at a speed of 25 centimeters per second. One channel recorded the forces while the other channel simultaneously recorded the wave surface profile directly over the center of the pipeline test section, thus allowing direct correlation of the two records.

The two-dimensional experimental data were digitized manually using a Gerber digital data reduction system connected with a card punch to automatically punch the digitized values on computer cards. Using a variable linear scale, each force record was first divided into 20 equally spaced intervals per wave, each interval representing a time interval of $T / 20$, where $T$ is the wave period. Each force record was digitized at these points over an interval of two consecutive waves (beginning at the wave crest), thus giving 40 values for the analysis and averaging the wave forces over two wave cycles. 


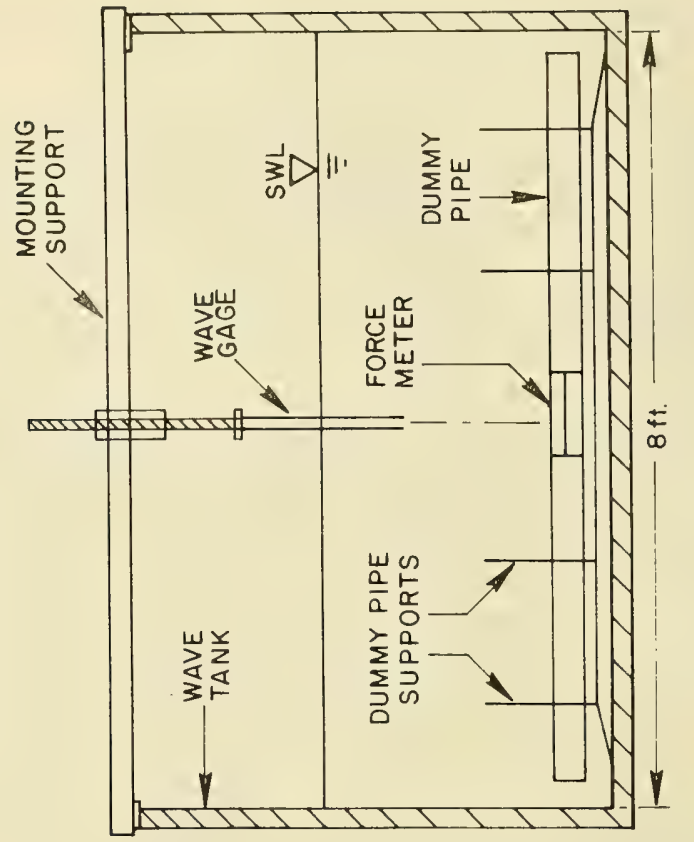

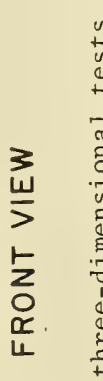

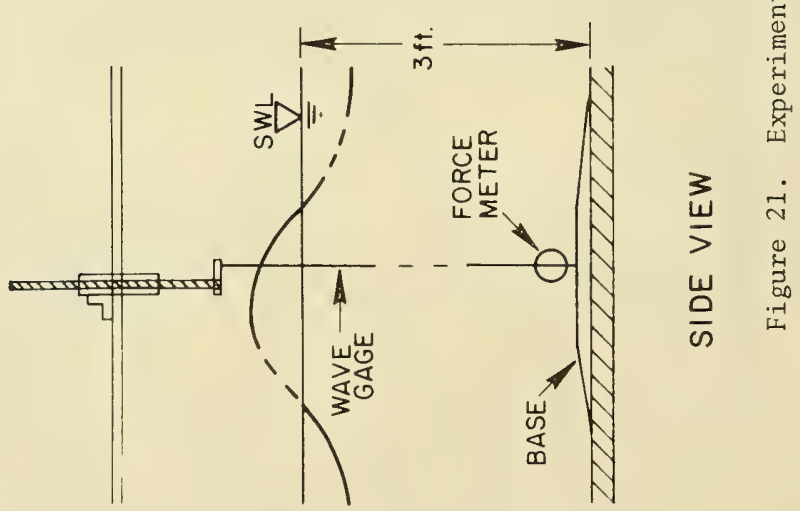




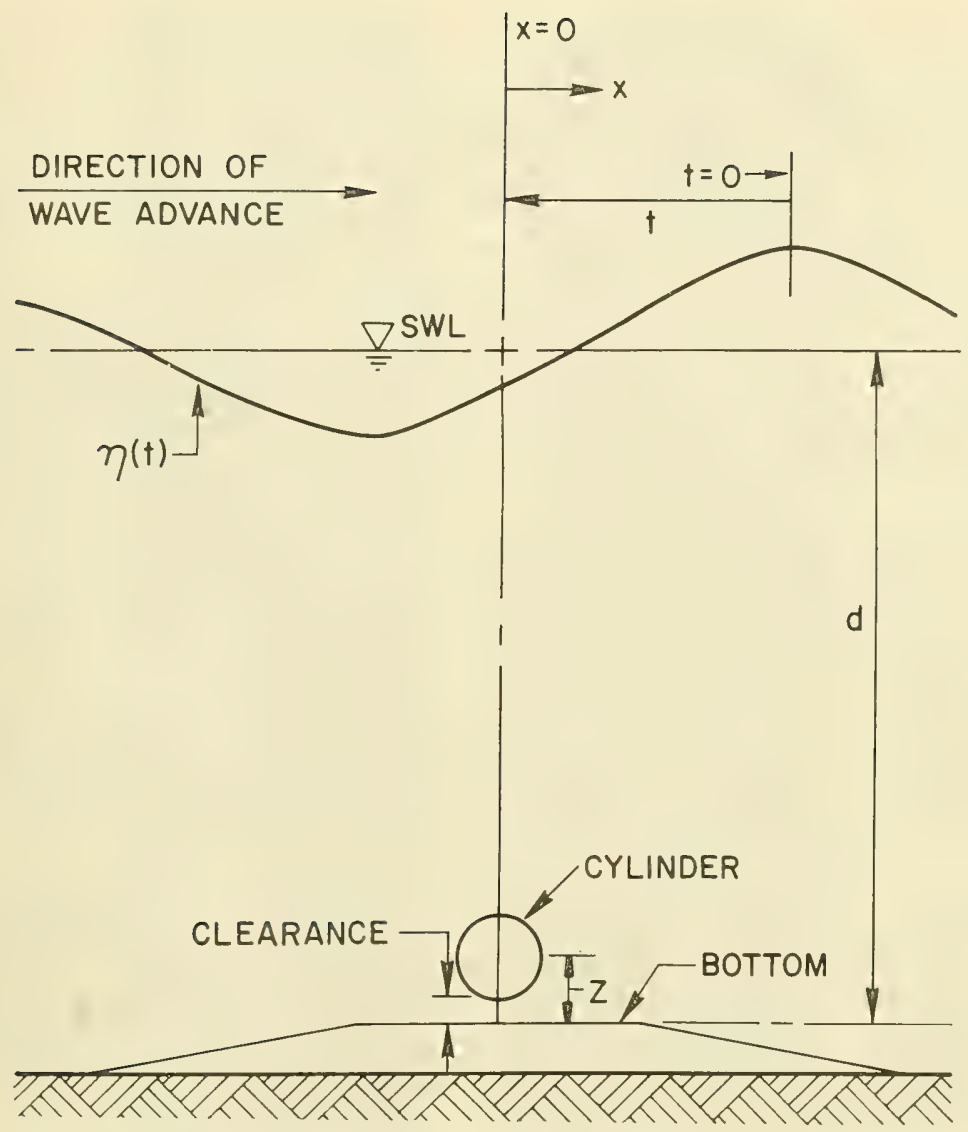

Figure 22. Definition sketch for three-dimensional experiments. 
The points in the force records corresponding to the wave crests were chosen as the origin (and end) of the digitized records. These points were determined by averaging the midpoints of three or four horizontal lines drawn through the crests of the wave record at several elevations above the stillwater level (SWL). These midpoints were approximately identical except for some of the larger, longer waves in which the peak of the wave crest did not exactly coincide with the midpoint of the zero crossings of the wave crest. A sample data record is given in Figure 23.

The three-dimensional experimental data were handled differently; the data were recorded on magnetic tape with an electronic digital data acquisition system. This instrument sampled alternatingly from the two channels (wave and force) at a rate of 100 samples per second, resulting in 50 samples per second from each channel.

The origin at the wave crest and the wave period were determined from the digitized wave records, rather than directly from the stripchart records. Since positive readings of the wave profile corresponded to the crest and negative readings corresponded to the trough, the point of origin of the wave crest was determined by taking the midpoint of the positive readings between zero crossings on the wave profile. The crest was thus defined as the data point closest to the midpoint of the zero crossings. The wave period was determined from the number of readings between two successive crests, since there was a time interval of $1 / 50$ second between each reading. Thus, the wave period was determined to the nearest 0.02 second.

The origin of the force record was taken as the force reading corresponding to the defined origin at the center of the wave crest surface profile. In reality, there was a small timelag of $1 / 100$ second between the wave profile readings and the corresponding force readings. This small timelag was ignored in the analysis, since it was felt that the accuracy of the defined origin at the wave crest was only good to the nearest $1 / 50$ second, the time interval between successive readings of the wave record.

Only one wave cycle was used for analysis of the electronically digitized data. Since the data were on magnetic tape, it was impossible to determine that two successive waves had exactly the same period and height until after the calculations were completed on the computer. Thus, if the waves had slightly different periods, the time phase correlation of the corresponding force readings would be slightly in error when taken over two wave cycles. In addition, since the accuracy, resolution, and rapid sampling rate of the electronic digitizer allowed more readings per wave cycle than the manual digitizing method, a sufficiently large number of force readings could be obtained in one wave cycle. 

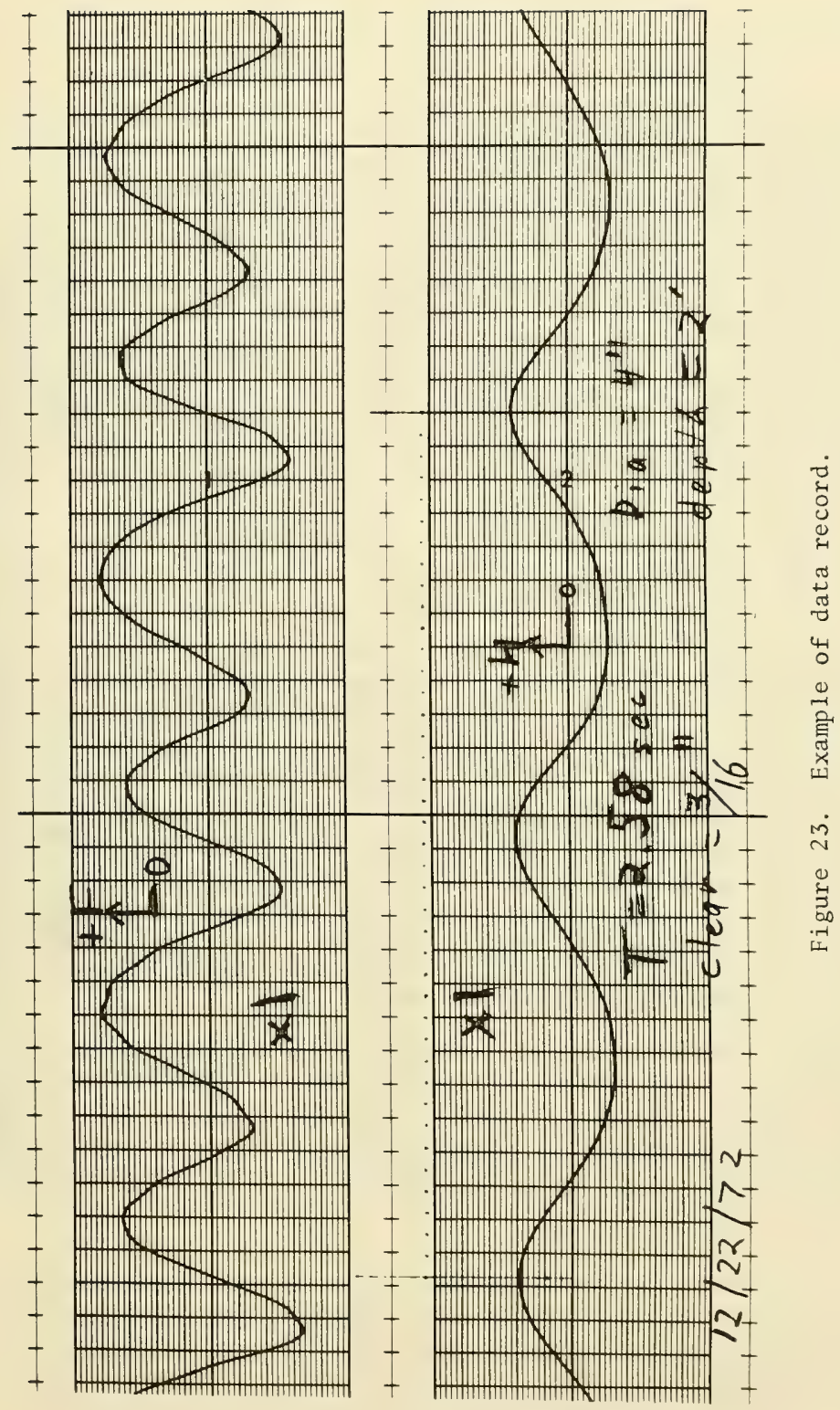
An estimation of the accuracy of the experimental measurements, along with the sources of error, is tabulated in the Table.

A least squares analysis was performed on the digitized force data to calculate the parameters, $C_{L}, \phi, k, C_{M}$, and $C_{D}$, of the vertical wave force equation, and the coefficients, $C_{M}$ and $C_{D}$, corresponding to the horizontal wave force equation. Using this approach, values of the wave force parameters that best fit the force data throughout the entire wave cycle can be determined. These values were then substituted back into the wave force equation to calculate the force over a complete wave cycle, thus allowing comparison of the results with the original data. The least squares analysis is given in Appendix A. The computer programs used for the analysis are given in Appendixes B, C, and D; the tabulated results of the analysis are in Appendixes E, F, and G. Examples of the computer output showing comparison of the original data with the forces calculated using the results of the least squares analysis are given in Figures 24 and 25 .

\section{RESULTS AND DISCUSSION}

\section{Resultant Force Through Wave Cycle.}

Both horizontal and vertical force measurements were made for some test conditions in the two-dimensional experiments using the 4-inchdiameter cylinder. The resultant force throughout the wave cycle could thus be determined for these conditions. Figures 26 to 32 show the resultant force plotted for each bottom clearance under the same wave condition, a period of 1.85 to 1.86 seconds and a wave height of 0.24 to 0.25 foot ( 7.32 to 7.62 centimeters). Values fxom the corresponding horizontal and vertical force records were plotted at 20 evenly spaced intervals $\left(18^{\circ}\right)$ through each wave cycle. The forces were plotted for two consecutive wave cycles to indicate the degree of scatter in the data. A rectangle was drawn at each plotted point to illustrate the horizontal and vertical range of the force data over the two wave cycles, and an envelope curve was drawn over these points.

Examination of these plots as a group (Fig. 33) shows the transition of the resultant wave-induced force with increasing clearance for the given wave condition ( $T=1.85$ to 1.86 seconds, $H=0.24$ to 0.25 foot) . The vertical component of the wave force is dominated by the lift force, while the horizontal component of the resultant force is due to the inertial and drag forces, with the inertial forces predominating for the experimental conditions tested.

For the smallest clearance $(0.001$ foot), in which the pipeline is almost in contact with the bottom, the resultant force attains a maximum upward value under the crests and troughs of the passing waves. The total wave force acts in the upward (positive) direction throughout the complete wave cycle, except for small downward forces in the vicinity of $90^{\circ}$ and $270^{\circ}$, where the horizontal flow reverses. 


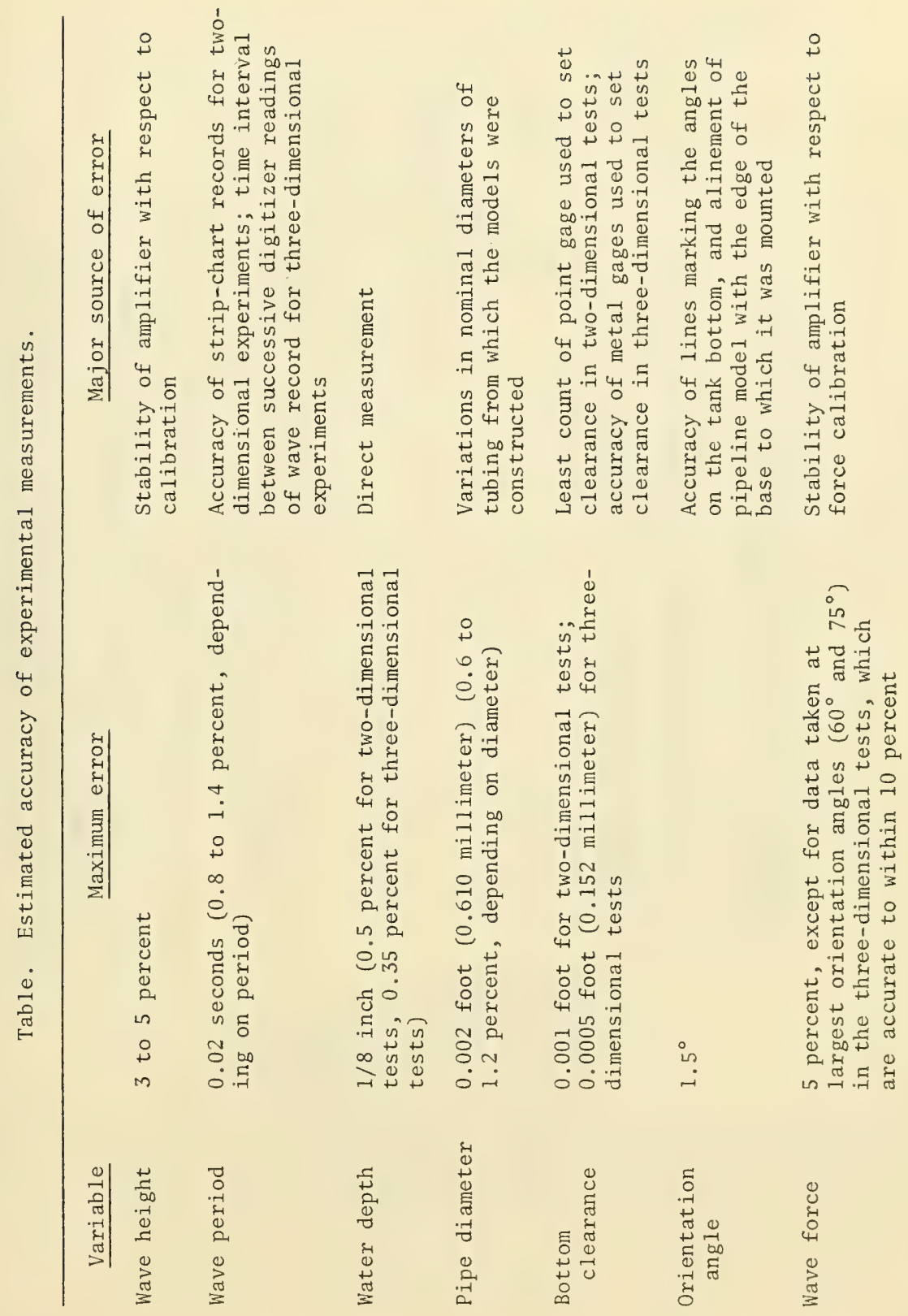




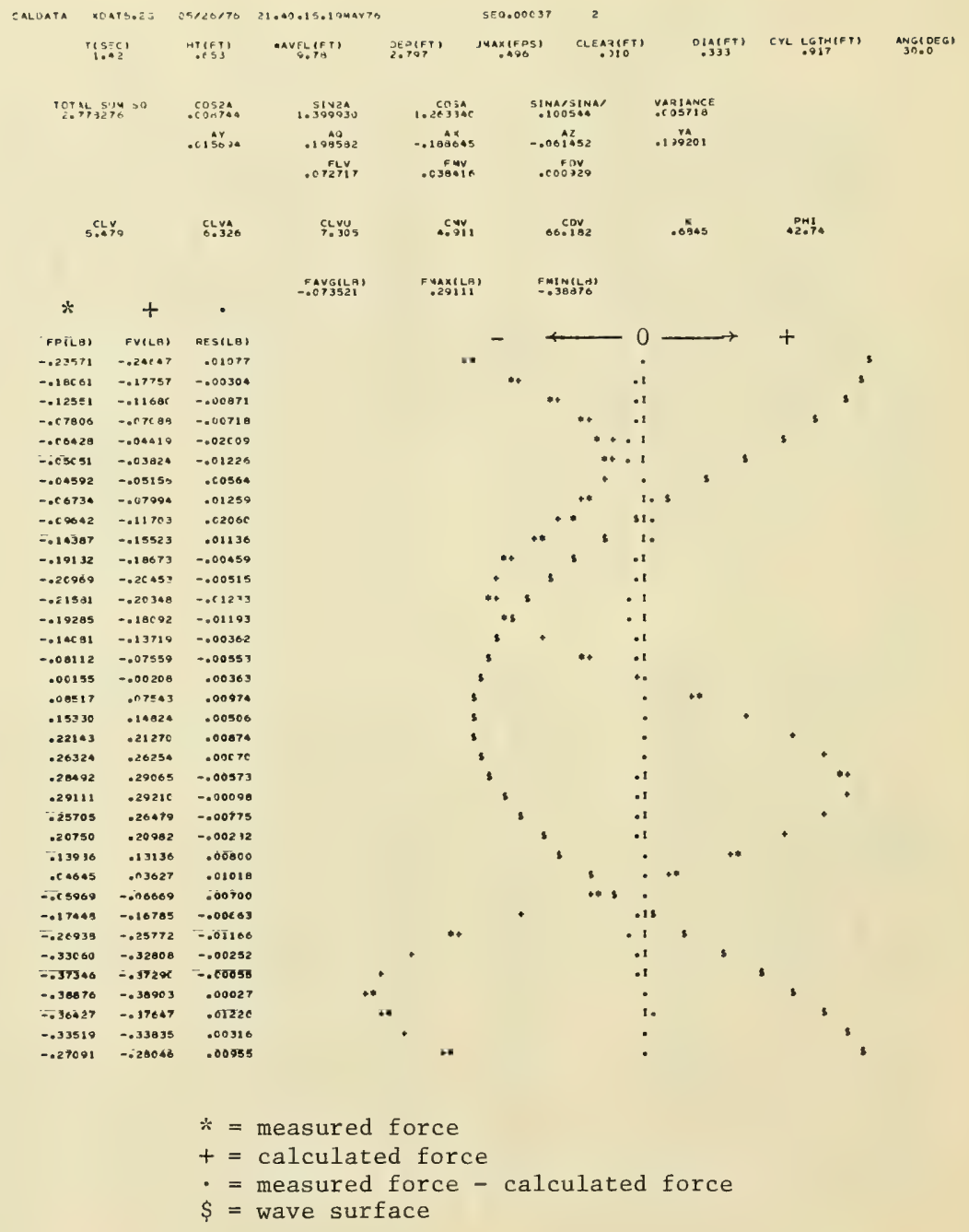

Figure 24. Example of computer output for vertical least squares analysis. 


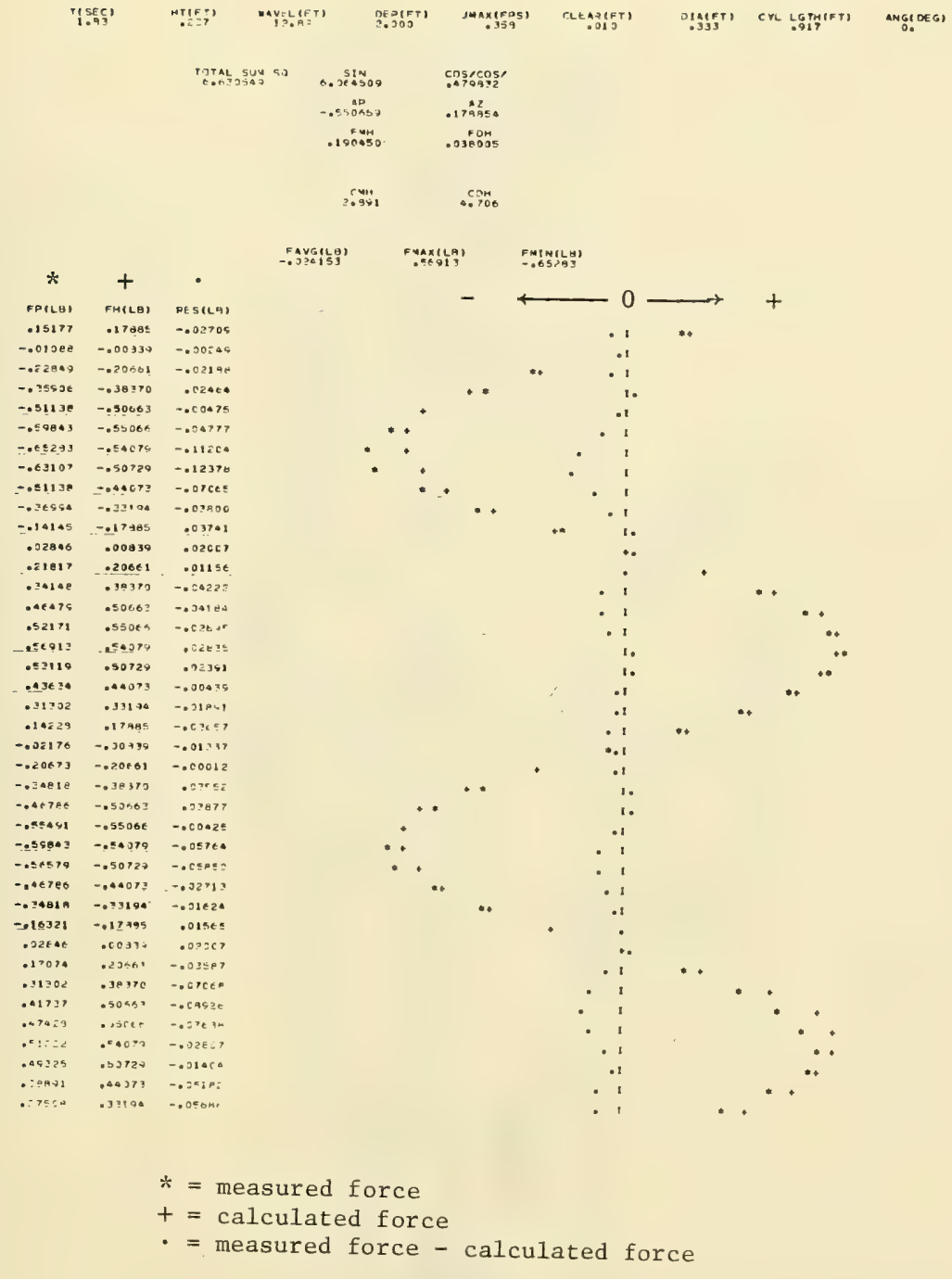

Figure 25. Example of computer output for horizontal least squares analysis. 


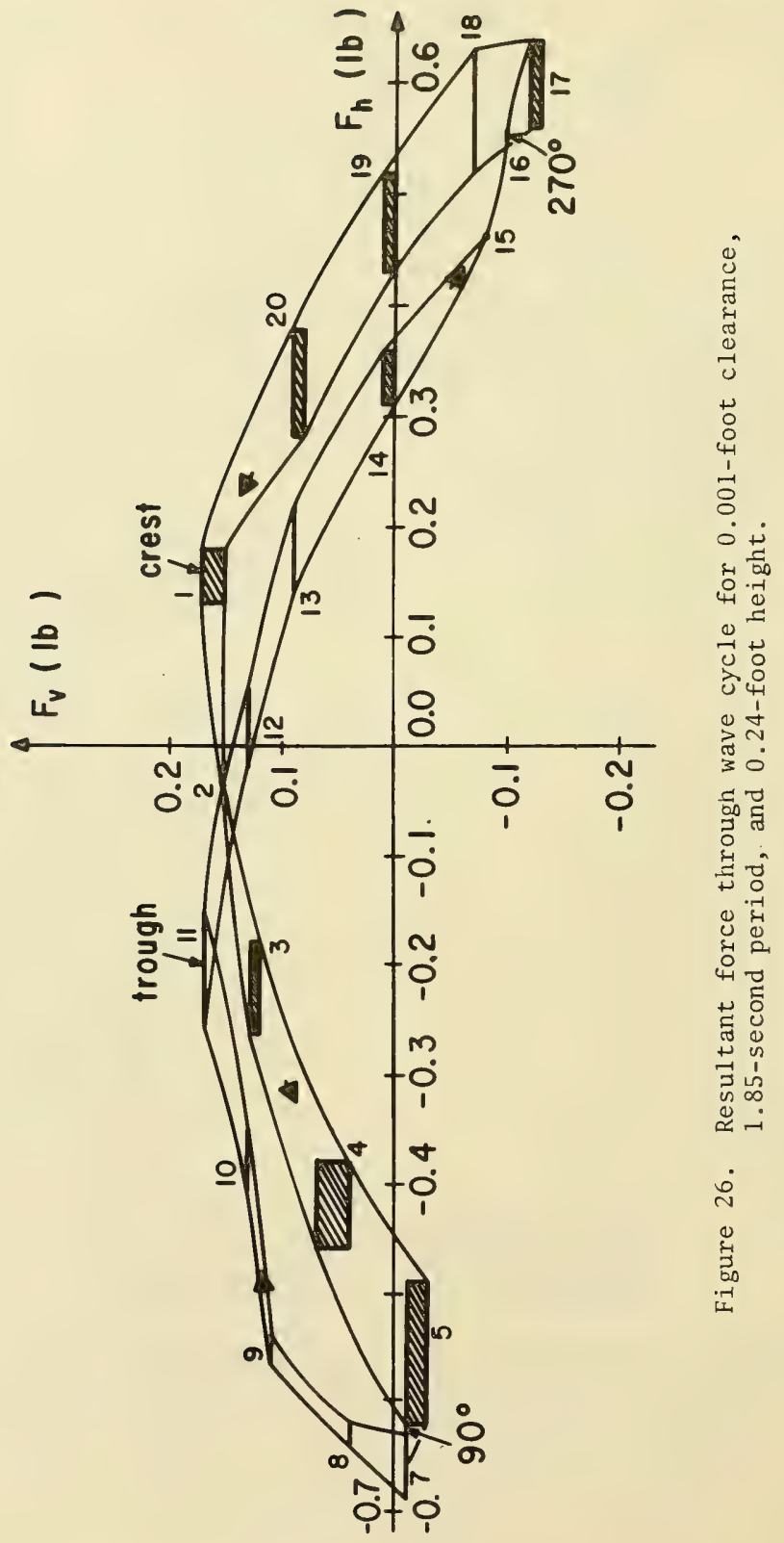

62 


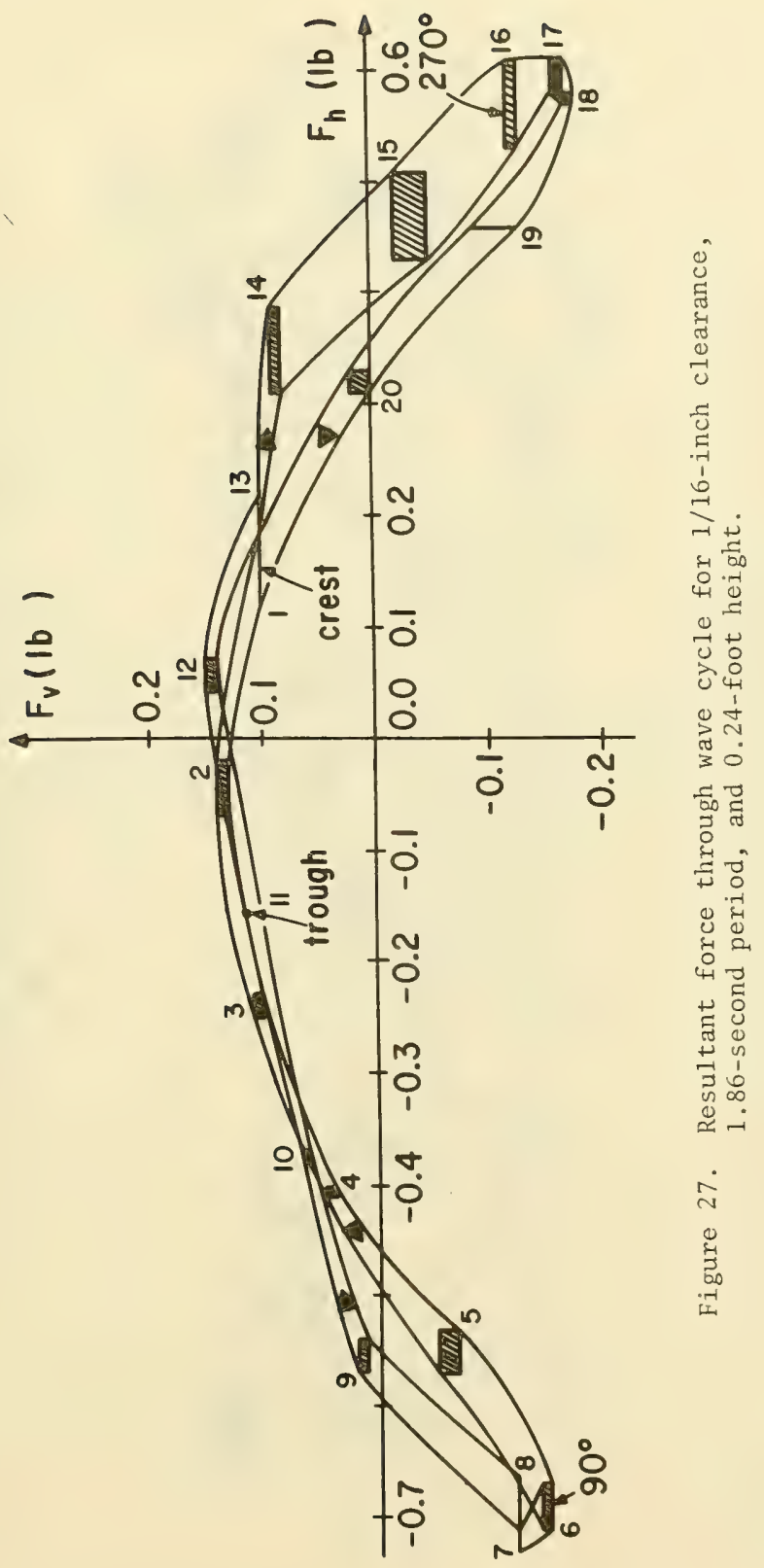




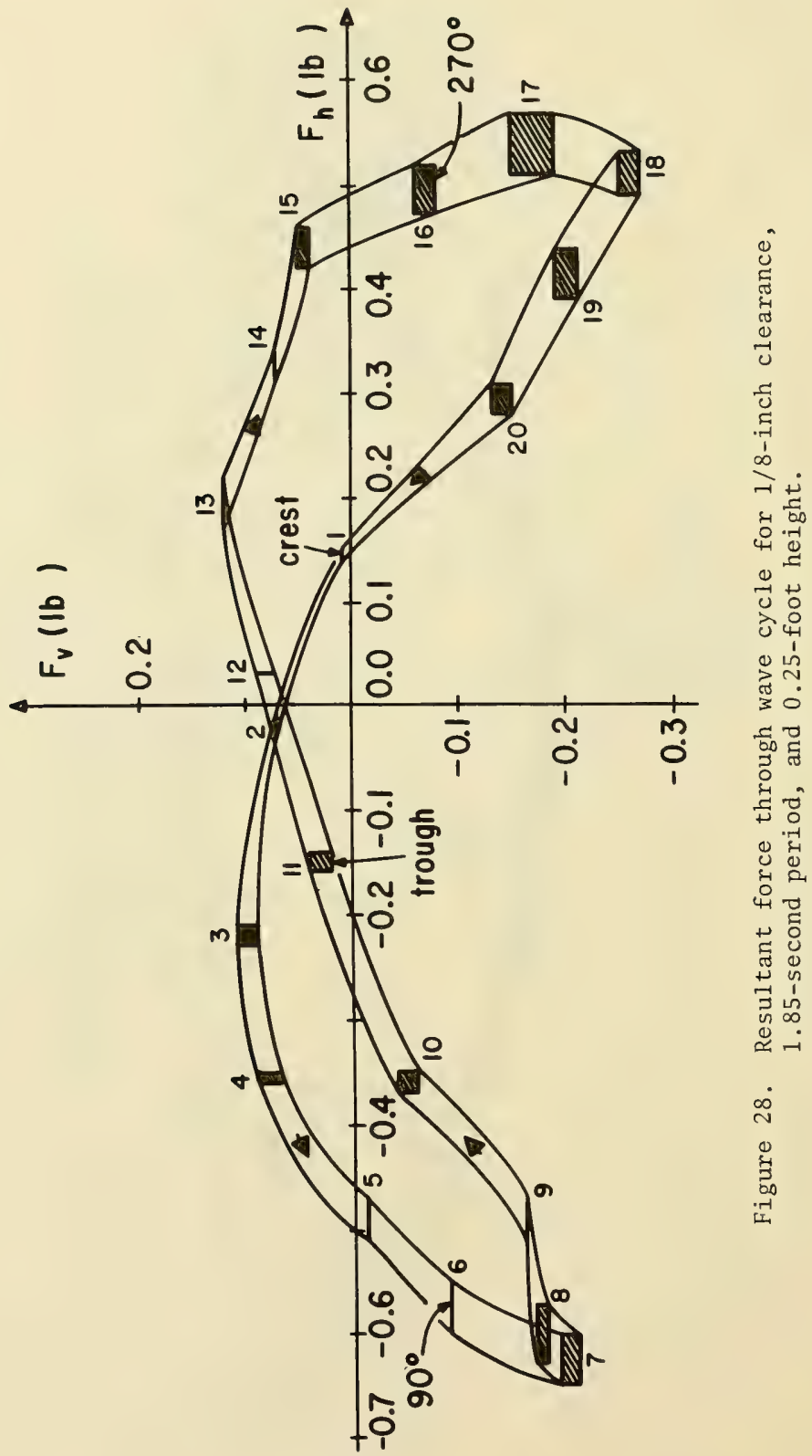




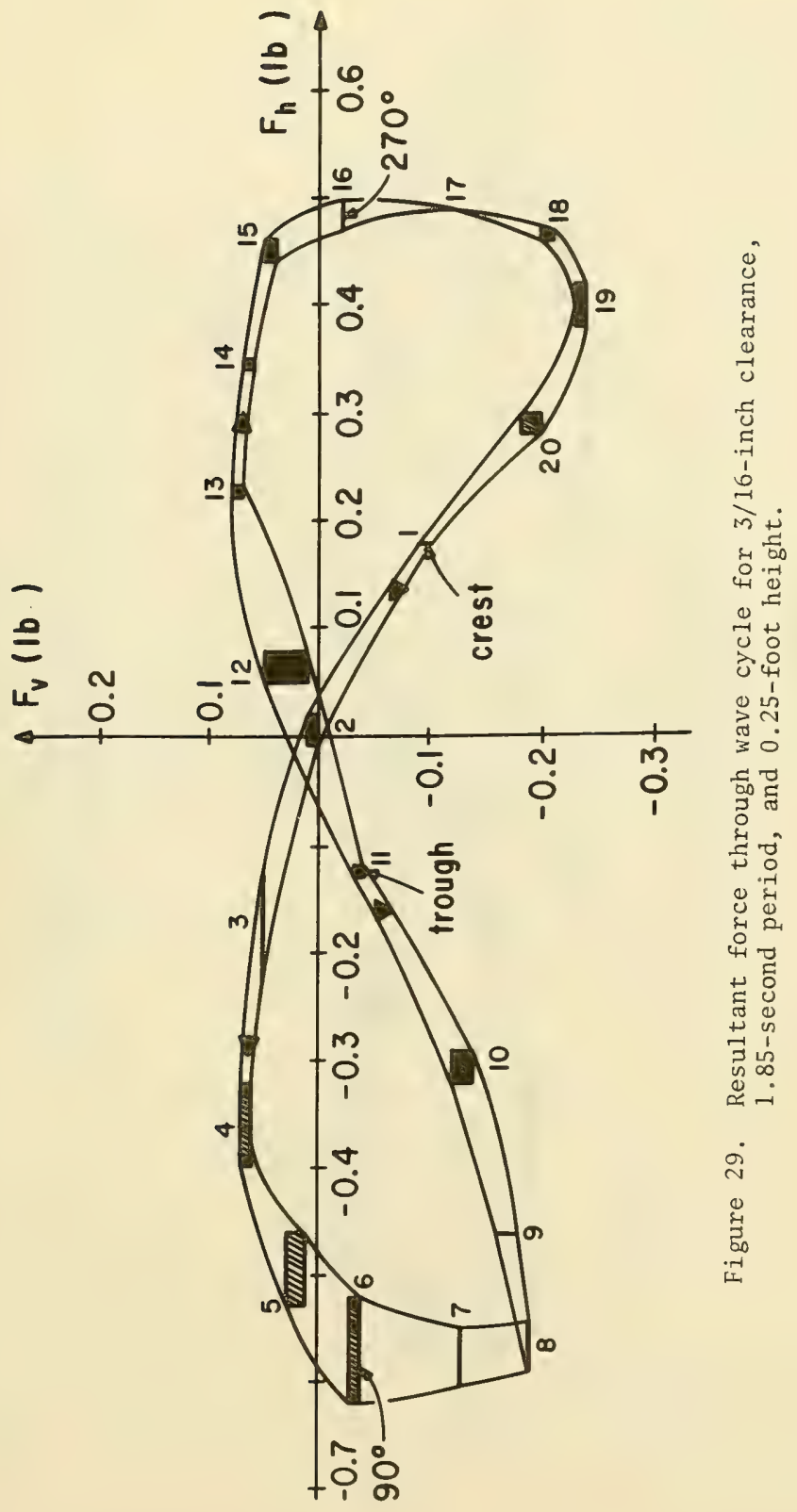




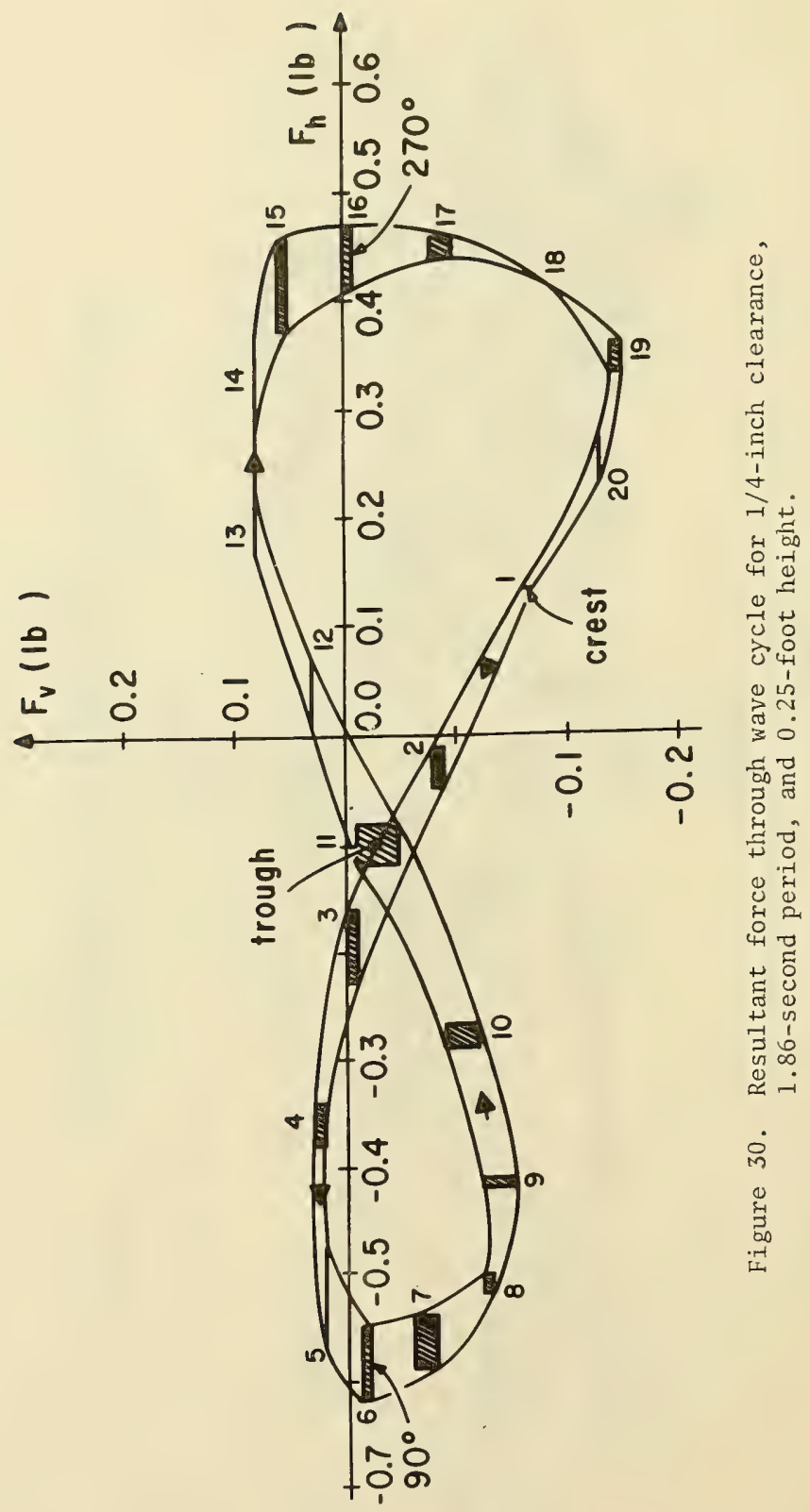




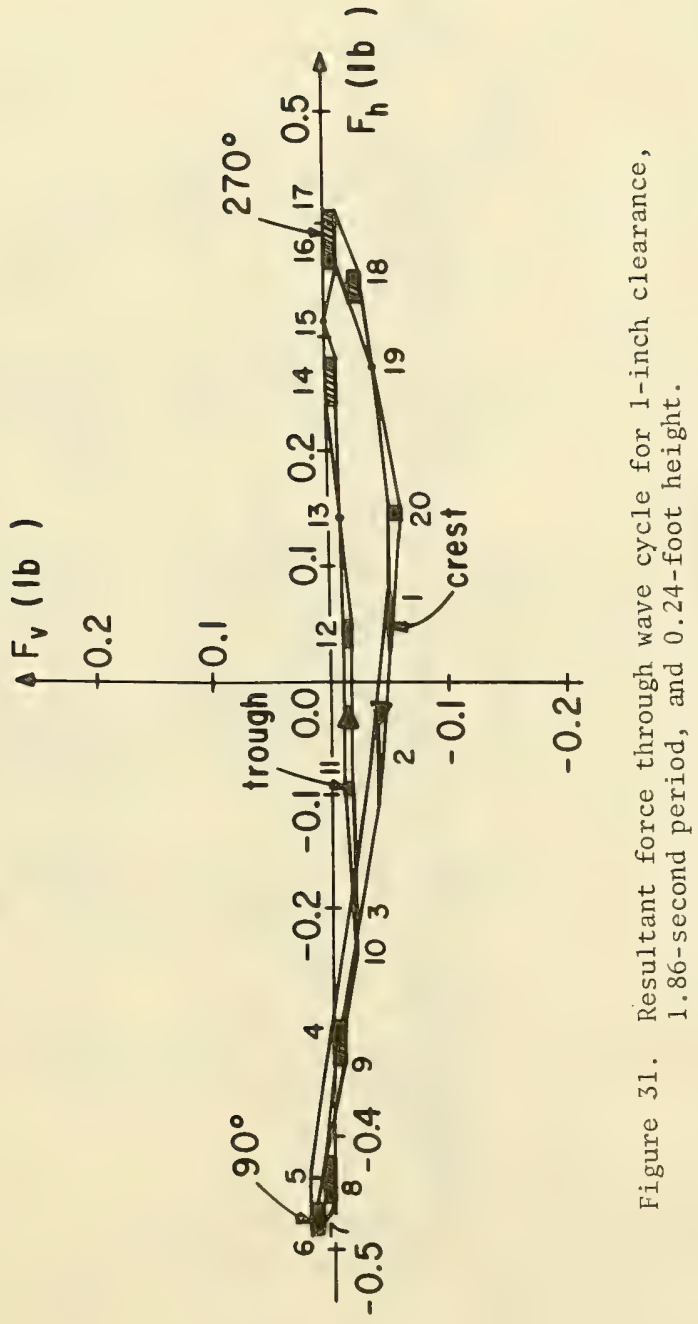




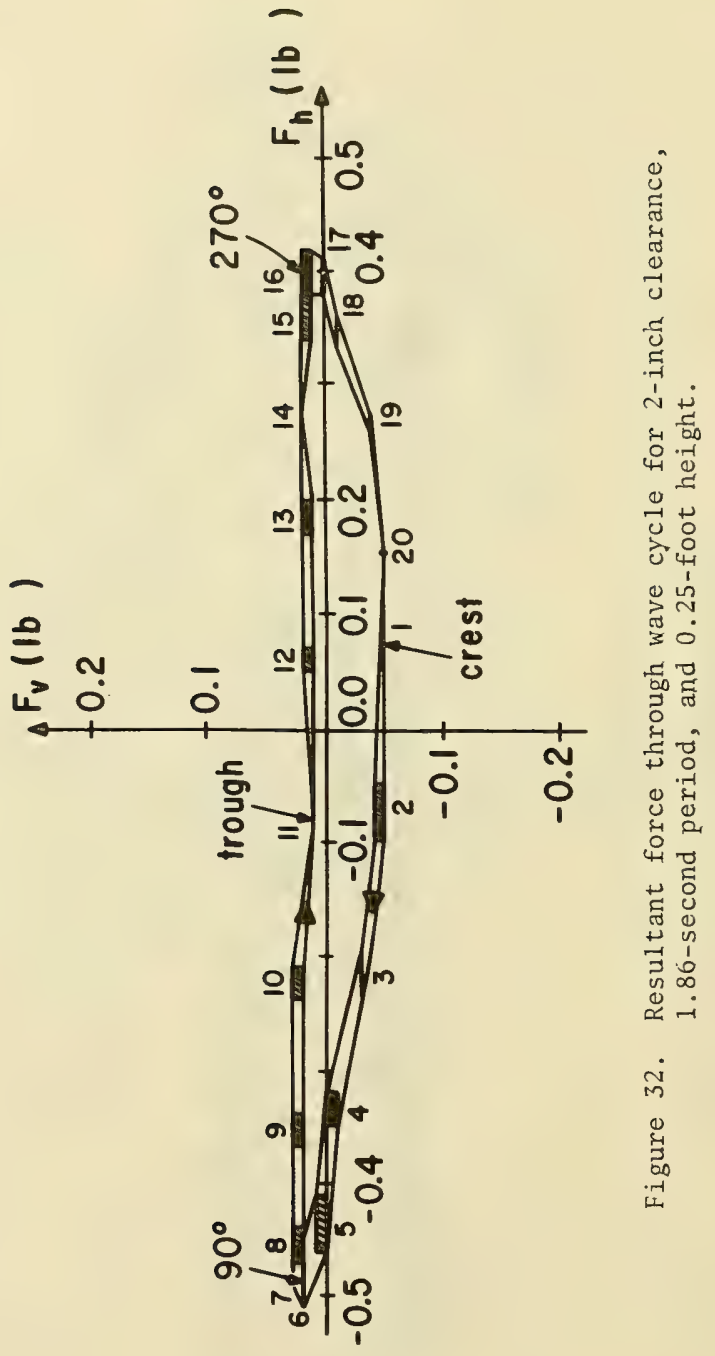




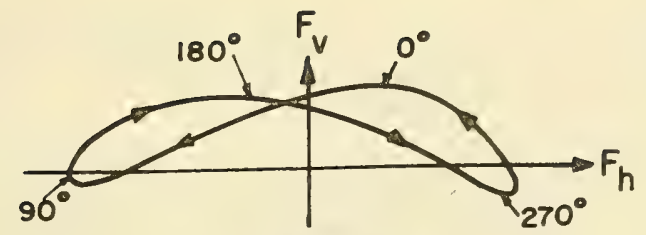

Clearance

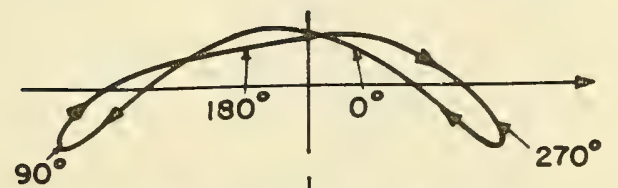

$\frac{1}{16}$ in

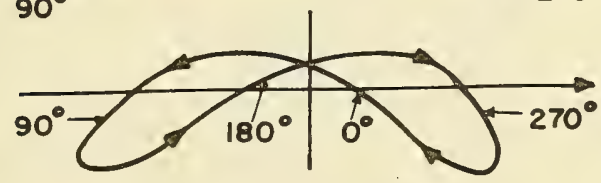

$\frac{1}{8}$ in

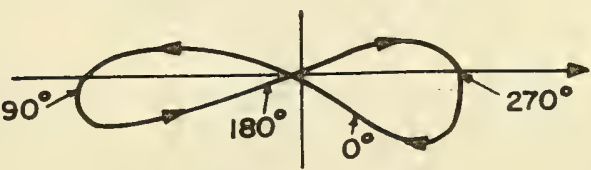

$\frac{3}{16}$ in

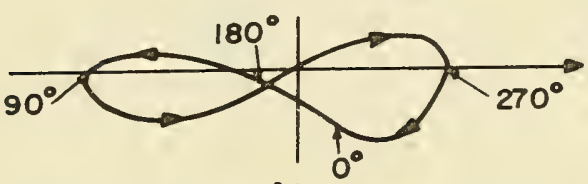

$\frac{1}{4}$ in

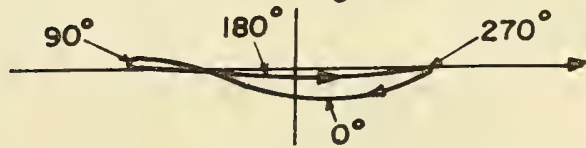

1 in

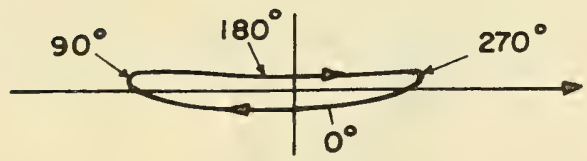

2 in

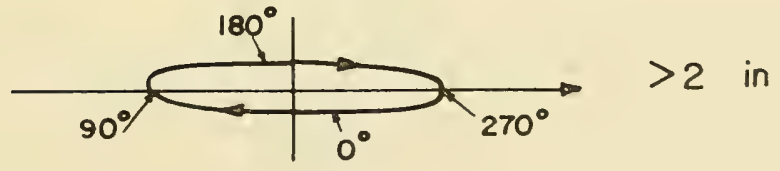

Figure 33. Change in resultant force with increasing clearance. 
As the clearance is increased to $1 / 16$ inch, the maximum upward forces decrease in magnitude, and also occur slightly later in the wave cycle. At the same time, the downward forces increase, reaching their maximum values at approximately $90^{\circ}$ and $270^{\circ}$.

Further increases in the bottom clearance produce a continuous shift of the positions of both the maximum upward and maximum downward forces later in the wave cycle. Simultaneously, the forces become downward rather tinan upward for a larger part of the cycle. At the same time, the vertical components of the wave force under the crests and troughs become negative and increase in the downward direction, while the negative forces at $90^{\circ}$ and $270^{\circ}$ gradually decrease to zero.

At a I-inch clearance, the resultant force acts downward throughout almost the complete wave cycle, with maximum downward forces occurring under the crests and troughs of the passing waves. The vertical forces are zero at $90^{\circ}$ and $270^{\circ}$, the positions of the maximum horizontal inertial forces. However, the lift effect is not very large for the 1-inch clearance. The resultant force plot for the 2 -inch clearance shows that the lift effect is still present, but is relatively small, even in comparison to the small vertical inertial forces.

At a slightly larger clearance, the lift effect will disappear, and the vertical forces will be due almost entirely to the inertial forces, since the vertical drag forces are negligible near the bottom. At this clearance, the inertial force will act upward under the trough and downward under the crest, so the resultant force plot will take the form of an approximately symmetrical ellipse. This condition is shown in Figure 34 for a smaller wave period ( 1.23 seconds), with a 1 -inch bottom clearance. The ellipse is distorted slightly, due to the small drag forces acting in the horizontal direction, $90^{\circ}$ out of phase with the larger horizontal inertial forces.

The horizontal components of the resultant wave force are also affected by the proximity of the bottom boundary. Although the horizontal water particle velocities and accelerations increase with distance above the bottom, the corresponding horizontal drag and inertial forces are larger when the pipe is close to the bottom than when it is located above at larger clearances.

Figures 35, 36, and 37 show the resultant force plots at both large and small bottom clearances, for a wave with a period of 0.95 to 0.96 second and a height of 0.24 to 0.25 foot. Because the wave period is smal1, the horizontal excursions of the water particles at the bottom and the duration of the horizontal flow are too small for the 1 ift effect to develop. So the forces acting in both the horizontal and vertical directions are mostly inertial, with a small drag component in the horizontal direction. The resultant force plots therefore take the form of an ellipse. 


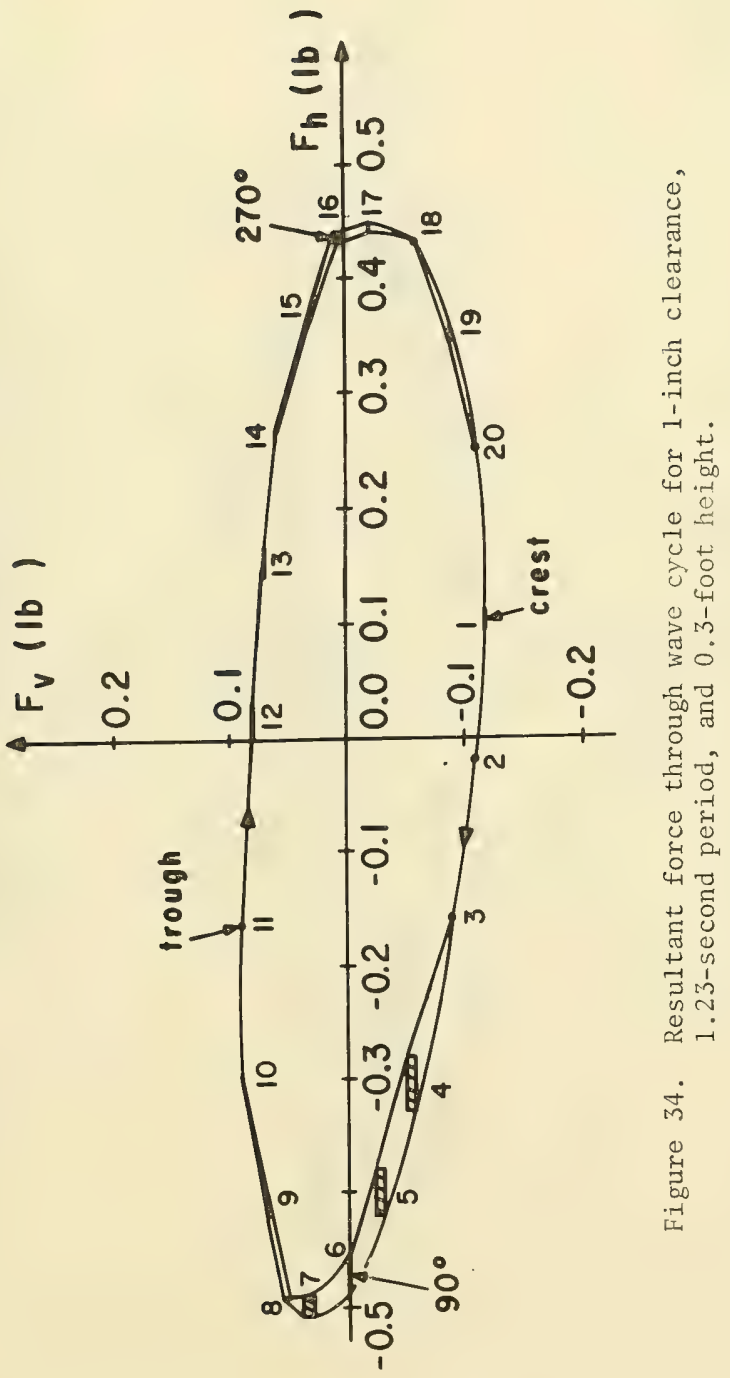




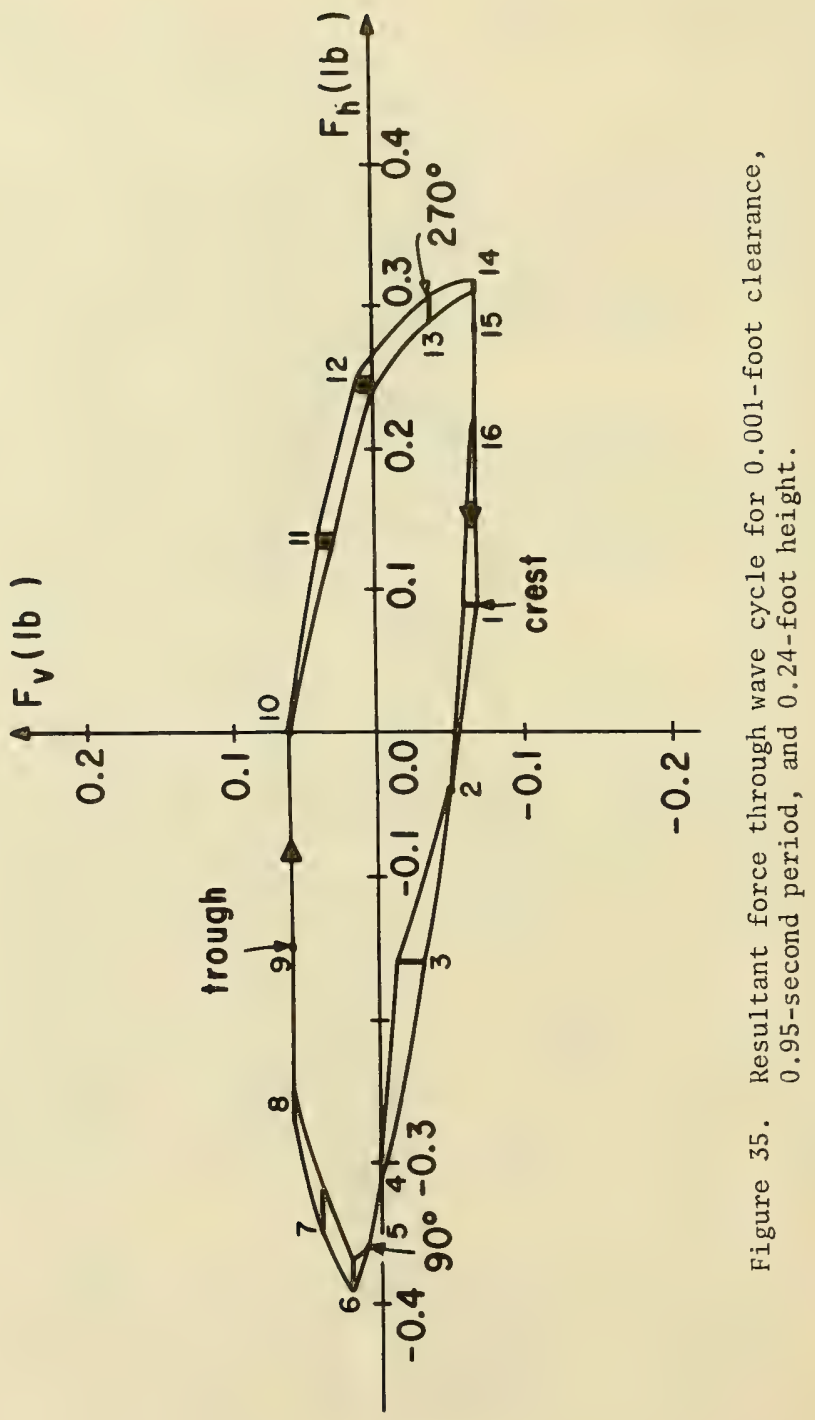




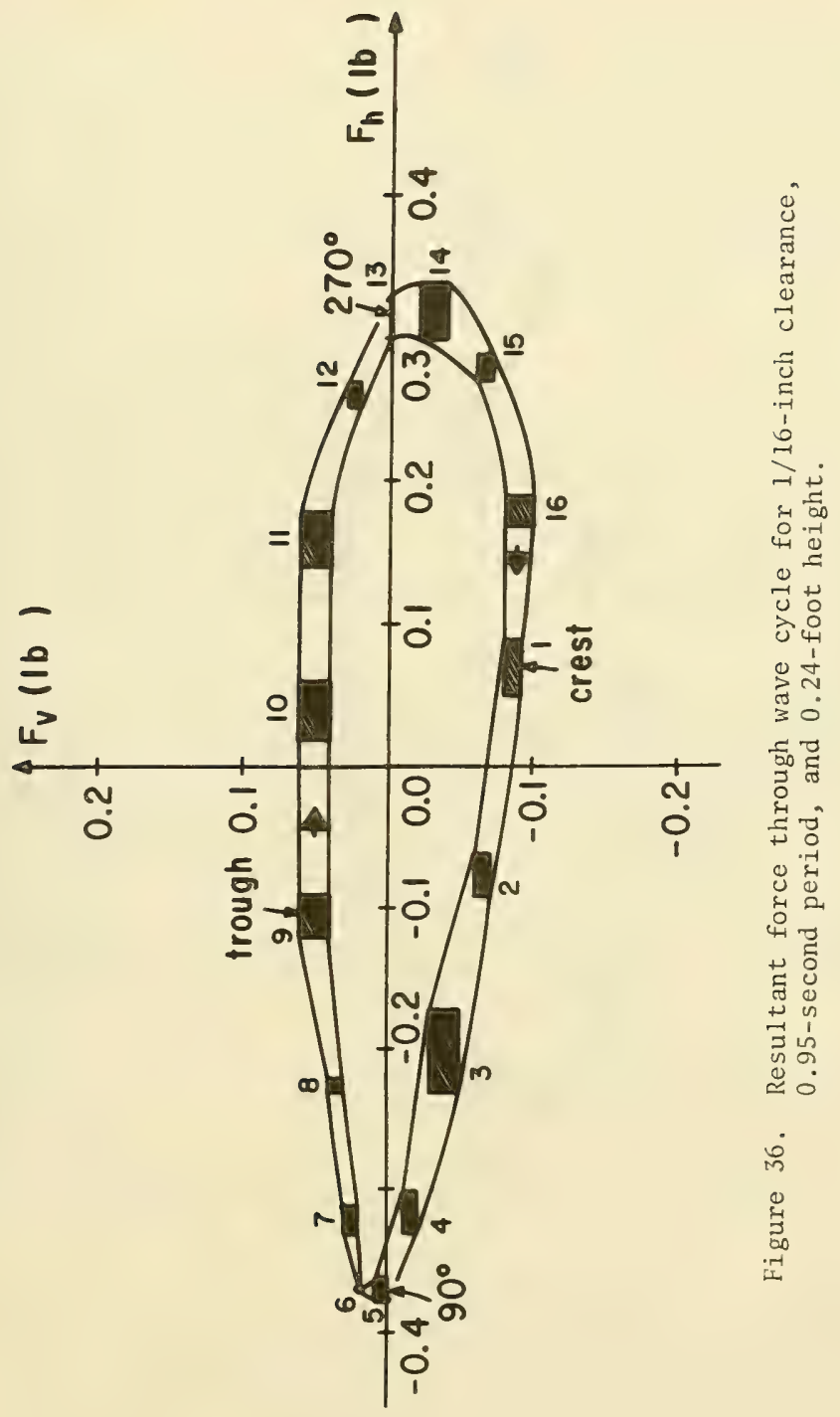




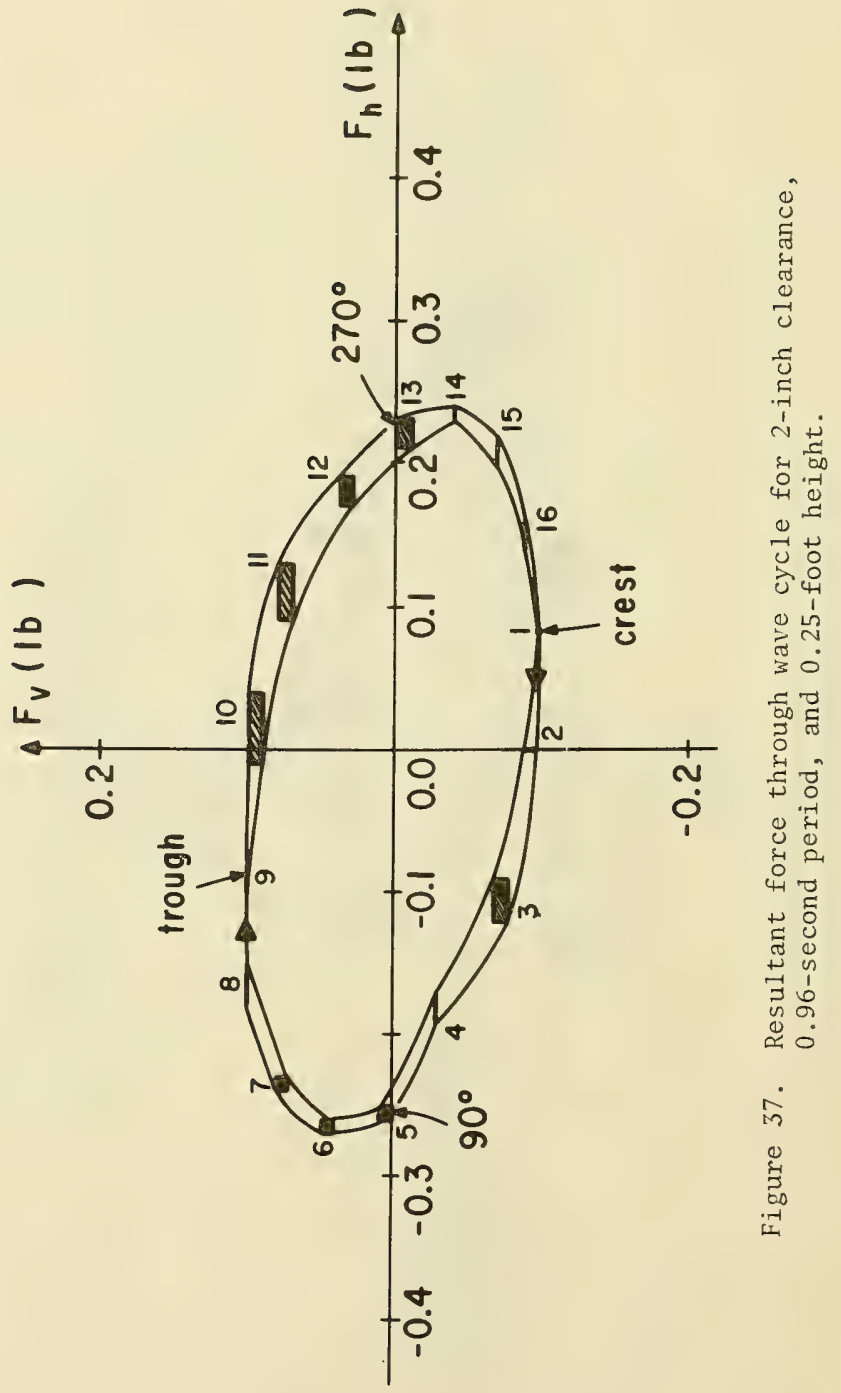


However, the horizontal components of the resultant forces are 1 arger at the smallest bottom clearances, even though the lift phenomenon is absent. The presence of the bottom boundary produces an asymmetric flow field around the pipeline. The resulting velocicies and accelerations of the water particles over the pipe section are thus modified by the presence of the boundary, and the associated horizontal forces are larger than they would be if subject to the same kinematics in the absence of the boundary. The increased horizontal forces on pipelines located close to the bottom are reflected in increased values of the coefficients of mass and drag, $\mathrm{C}_{\mathrm{M}}$ and $\mathrm{C}_{\mathrm{D}}$.

\section{Orientation Angle Considerations.}

The coefficient of lift calculated in the least squares analysis of the experimental data was computed using two alternative approaches (Fig. 38): (a) the total horizontal water particle velocity in the direction of wave advance, with the projected area of the pipeline in the plane perpendicular to the direction of wave advance; and (b) only the component of the horizontal water particle velocity perpendicular to the pipeline axis, with the projected area in the plane parallel to the pipeline axis.

After tabulating the data from the three-dimensional experiments, it became apparent that the second method gave consistent values of the coefficient of lift for all angles of orientation. In contrast, the values of $\mathrm{C}_{\mathrm{L}}$ obtained using the first method gave values that were $10 \mathrm{w}$, and which decreased with increasing angles of orientation (where $0^{\circ}$ corresponds to a pipeline parallel to the wave crests).

Relationships between the coefficient of 1 ift, $\mathrm{C}_{\mathrm{L}}$, and the parameters, $\phi$ and $k$, of the lift force equation were the same for all angles of orientation when $\mathrm{C}_{\mathrm{L}}$ was calculated considering only the component of the horizontal velocities perpendicular to the pipeline axis.

In addition, relationships involving any of the parameters of the lift force equation $\left(\mathrm{C}_{\mathrm{L}}, \phi\right.$, or $\left.\mathrm{k}\right)$ and various dimensionless parameters defining the wave and pipeline conditions were consistent for all angles of orientation when the horizontal water particle velocity acting on the pipe section was treated by considering only the component perpendicular to the pipeline, and completely ignoring the parallel component.

Thus, the results of this investigation show that the modified lift force equations presented in this report can be applied to pipelines located at any angle of orientation with respect to the wave crests. However, only the component of the horizontal water particle velocity perpendicular to the pipeline axis should be considered as contributing to the wave-induced lift force acting on the pipeline. Using this approach, the parameters, $\mathrm{C}_{L}, \phi$, and $\mathrm{k}$, defining the lift forces exhibit the same quantitative relationships between the various dimensionless parameters defining the wave and pipe conditions, regardless of the angle of orientation. 

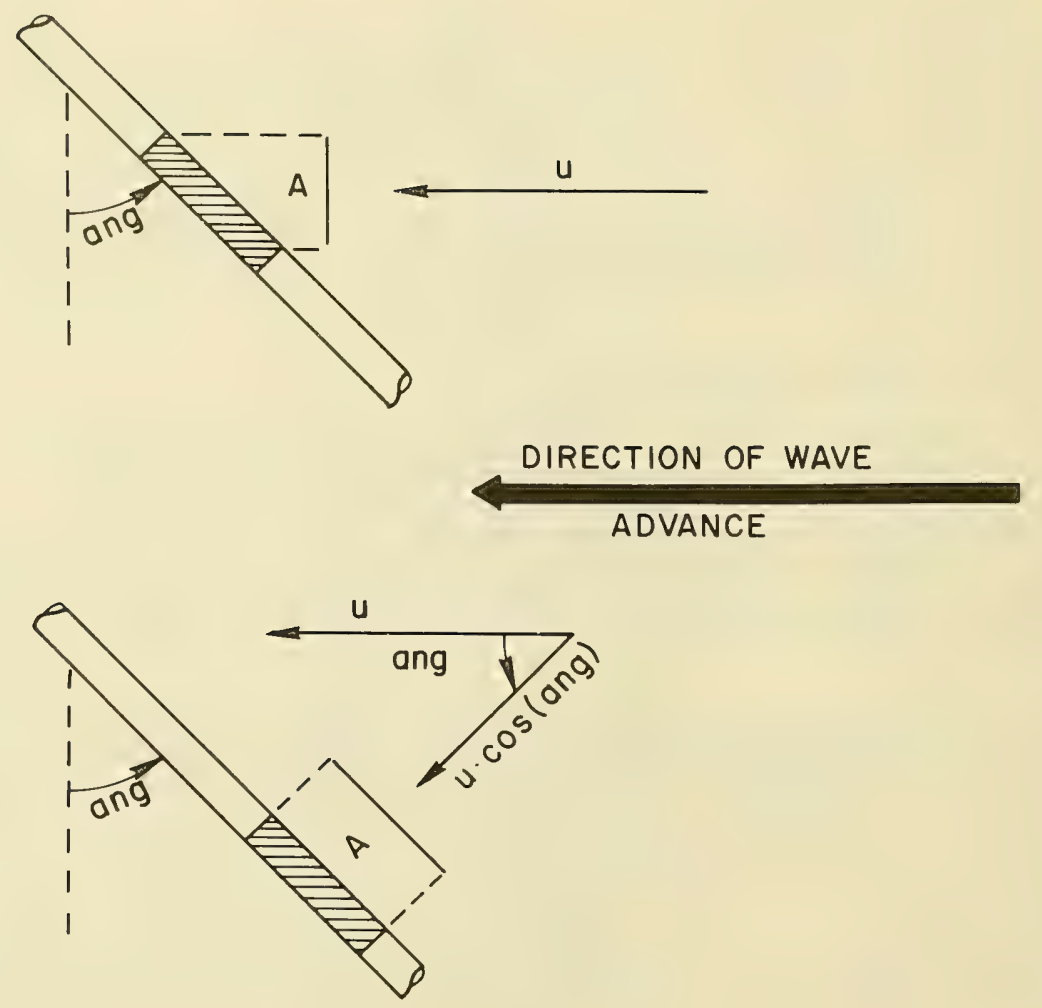

Figure 38. Alternative approaches for handling pipeline orientation angles. 
$\phi$ and $\mathrm{k}$ were defined as varying from $0^{\circ}$ to $90^{\circ}$ and 0 to 1 , respectively, with increasing clearance. $\phi=0^{\circ}$ and $\mathrm{k}=0$ correspond to the case of a pipeline in contact with the bottom (no clearance), while the maximum values of $\phi=90^{\circ}$ and $\mathrm{k}=1$ correspond to the case of a large enough clearance so that the choking phenomenon does not occur at any time througiout the wave cycle. Since a simultaneous increase of both parameters was noted in the data for increasing clearance between the two limiting cases, it was suspected that a direct relationship may exist between $\phi$ and k. Such a relationship was found, as shown in Figure 39. The same relationship held for all three pipe diameters tested, regardless of the orientation angle, indicating that the relationship was independent of these two factors, and was thus valid for any pipeline configuration in which the lift effect was present.

In this plot and the ones that follow, the data for orientation angles from $0^{\circ}$ to $30^{\circ}$ were plotted for each pipe diameter, without differentiating the data corresponding to each angle. The relationships shown were found to be valid regardless of the angle of orientation, provided the data were handled as discussed above (using the component of the horizontal velocity perpendicular to the pipeline axis). The data corresponding to each pipe diameter are distinguished by using different plot symbols. The same relationships hold for orientation angles of $45^{\circ}$, but these data were not plotted in order to minimize scatter so that differences between the pipe diameters could be detected more easily. In general, the same relationships held for orientation angles up to $60^{\circ}$. But in some cases, the Iift effect was negligible at high orientation angles, so the values of the associated parameters $\left(\mathrm{C}_{\mathrm{L}}, \phi\right.$, and $\left.\mathrm{k}\right)$ were less accurate. Thus, plotting all of the data corresponding to the larger orientation angles would introduce additional scatter, obscuring the valid relationships which were consistent when the lift forces were significant.

A relationship was found between the coefficient of 1 ift, $\mathrm{C}_{\mathrm{L}}$, and the parameters, $\phi$ and $\mathrm{k}$ (Figs. 40 and 41). $\mathrm{C}_{\mathrm{L}}$ appears to be better correlated with $\mathrm{k}$ than with $\phi$. Note that for minimum values of $\mathrm{k}$ and $\phi$, corresponding to the case of a pipeline in contact with the bottom, the value of $\mathrm{C}_{\mathrm{L}}$ is approximately 4.5. This value is of interest, since it agrees with the potential flow solution $\left(C_{L}=4.495\right)$ for the value of the coefficient of lift for a circular cylinder in contact with a plane wall, subject to an inviscid steady flow (Yamamoto, Nath, and Slotta, 1973).

Maximum values of $\mathrm{C}_{\mathrm{L}}$ occur at approximately $\mathrm{k}=1 / 2$, corresponding to maximum lift forces that are equal in both the upward and downward directions. The average value of the coefficient of lift at this point is about 9.0 , with values extending up to about 10.5 . These maximum values of $C_{L}$ are attained at approximately $\phi=25^{\circ}$ to $30^{\circ}$ in the $\phi$ versus $C_{L}$ plot.

Since the coefficient of lift, $C_{L}$, defines the combined magnitude of both the positive and negative lifts, it can be separated into two parts: (a) the part defining the magnitude of the positive lift, $C_{L}(I-k)$, and 


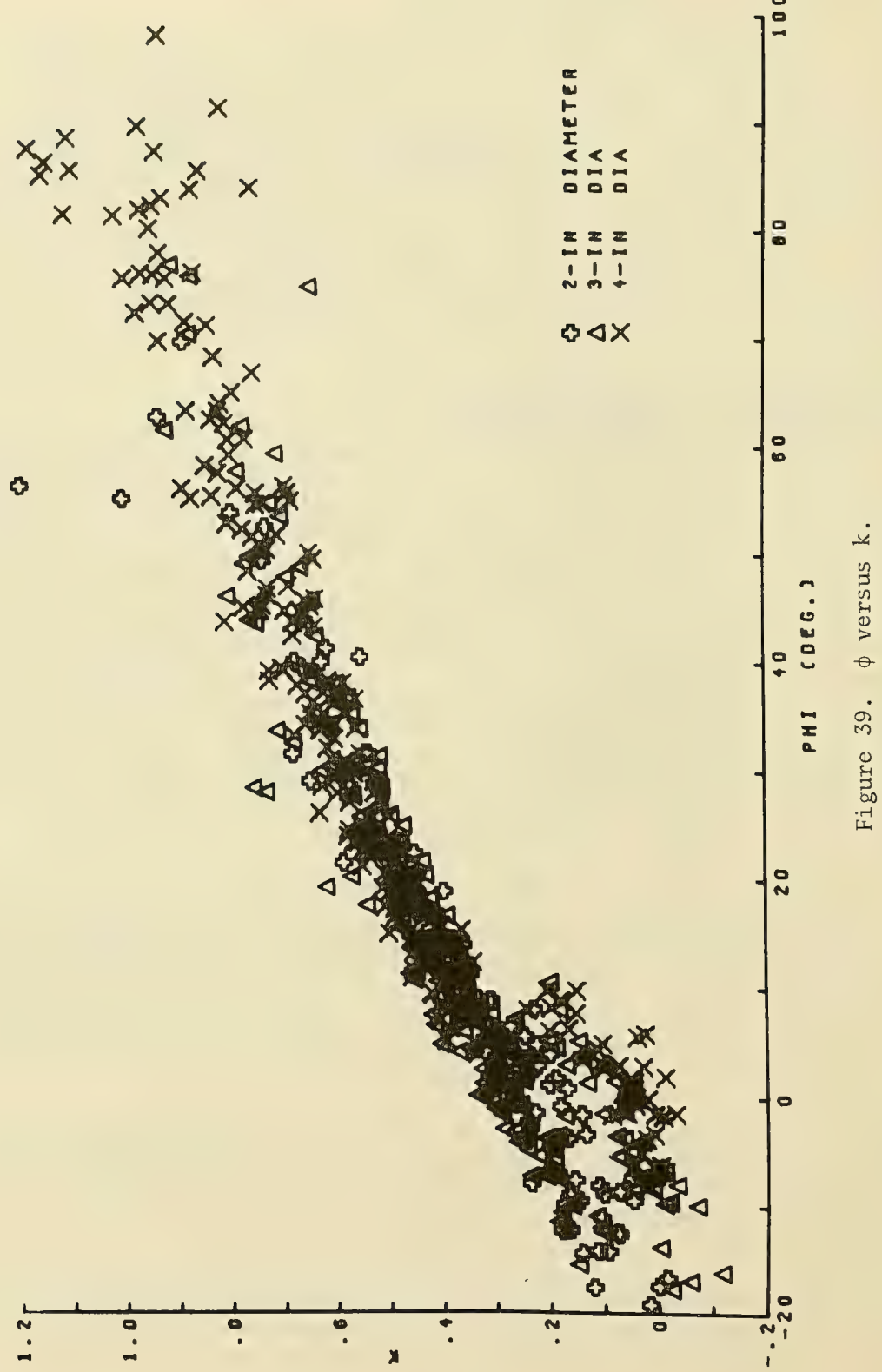




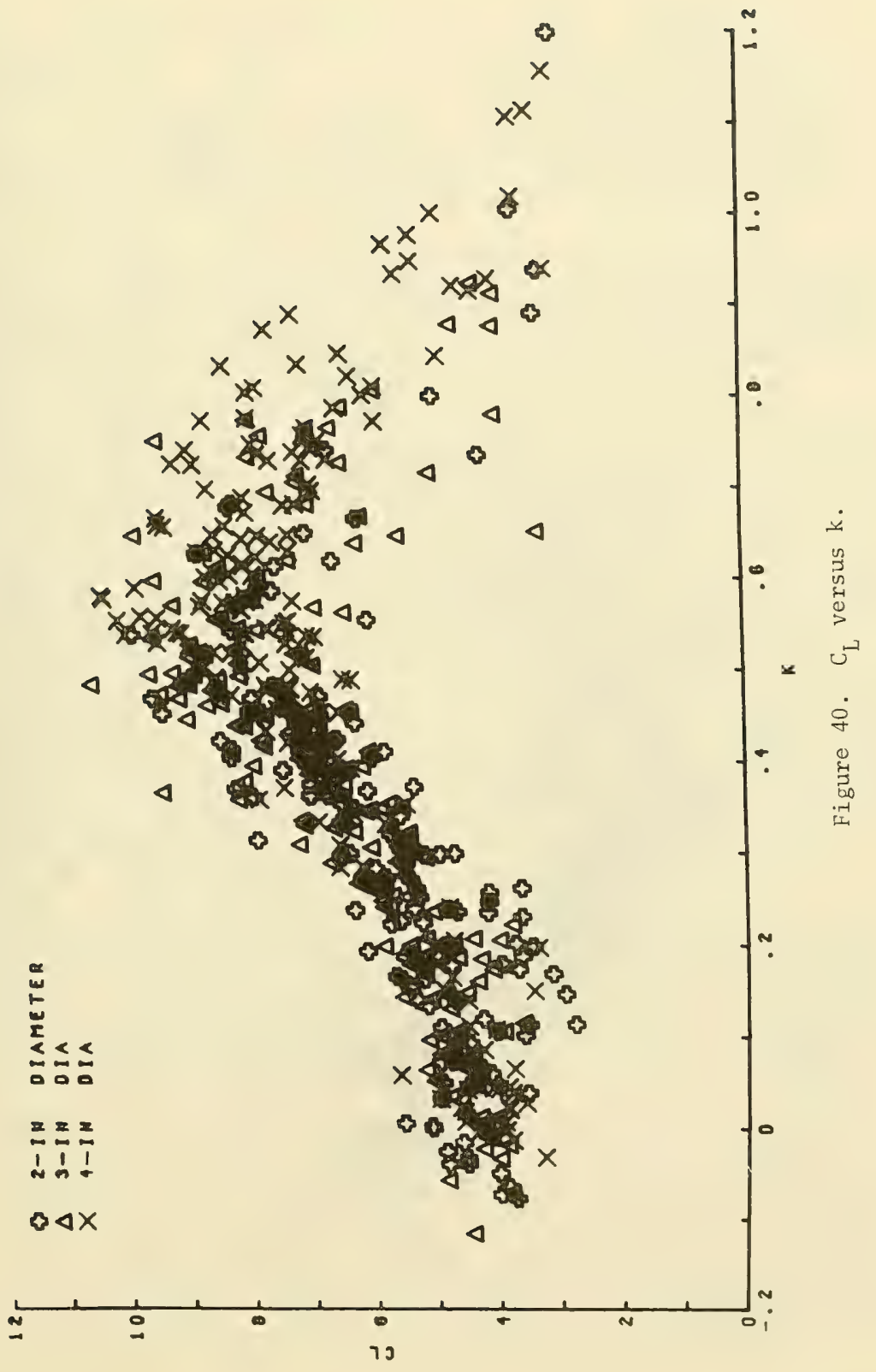




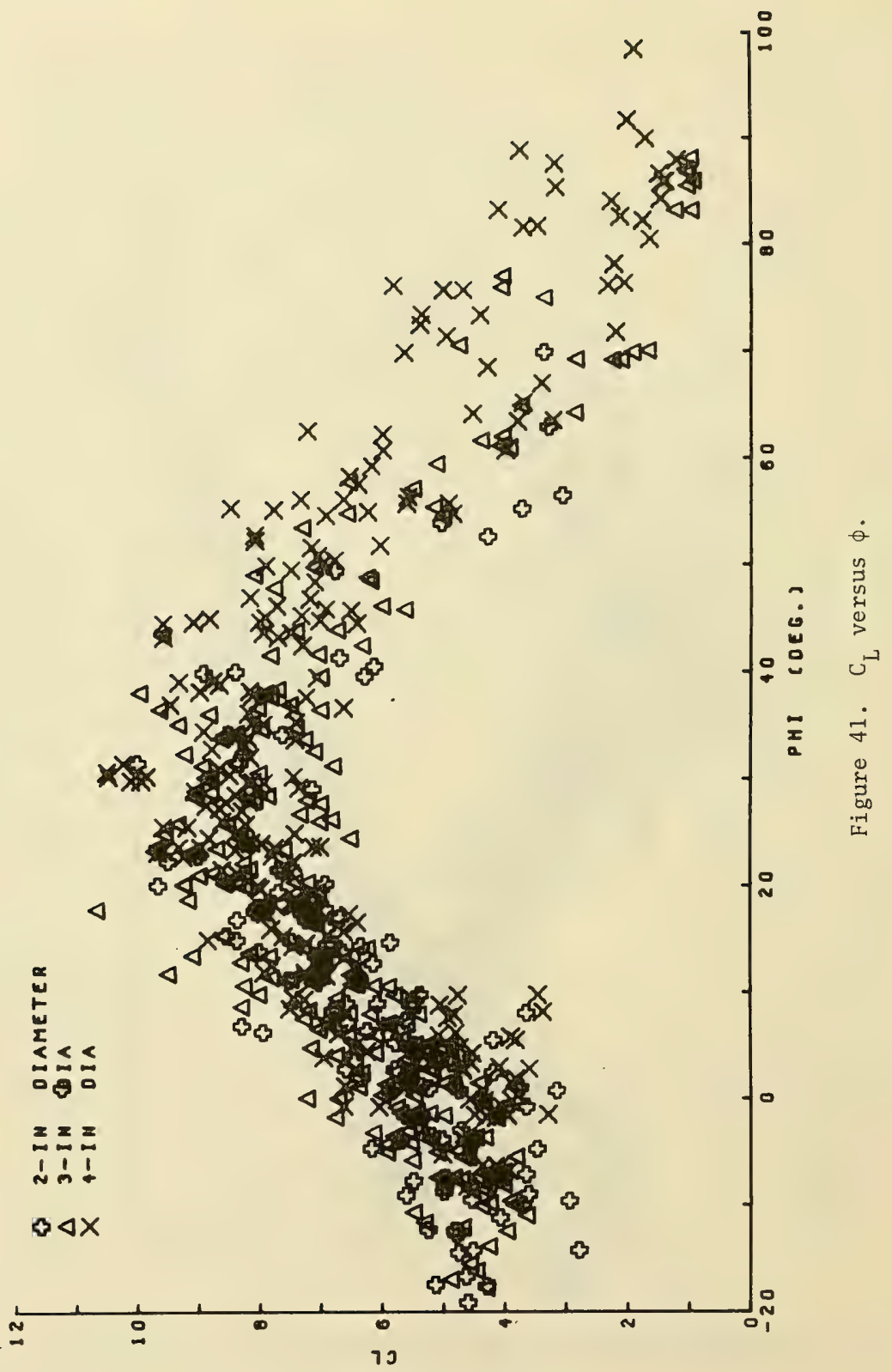


(b) the part defining the magnitude of the negative lift, $C_{L}(k)$. The quantities, $C_{L}(1-k)$ and $C_{L}(k)$, can be referred to as the effective positive coefficient of lift and the effective negative coefficient of lift, respectively. Since $C_{L}=9.0$ for $k=1 / 2$, both $C_{L}(1-k)$ and $C_{L}(k)$ are equal to 4.5 at this point. This means that the lift forces can reach the same maximum magnitude in both the upward and downward directions as are attained in the upward direction only for the same pipe in contact with the bottom (where $C_{L}(1-k)=4.5$, but $C_{L}(k)=0$ ).

The effective positive and negative coefficients of lift are plotted versus both $\phi$ and $k$ in Figures 42 to 45 . Again, the correlations are much better with $k$ than with $\phi$. The average value of $\mathrm{C}_{\mathrm{L}}(1-\mathrm{k})$ drops on $1 y$ slightly between $k=0$ and $k=1 / 2$, but for values of $k$ greater than $1 / 2$, the effective positive coefficient of lift drops rapidly to a value of 0 when $\mathrm{k}=1$.

The average value of $\mathrm{C}_{\mathrm{L}}(\mathrm{k})$ increases with $\mathrm{k}$ until it reaches a maximum value of about 6.0 when $\mathrm{k}=0.75$, and then decreases to about 4.5 when $\mathrm{k}=1$. Individual maximum values of $\mathrm{C}_{\mathrm{L}}(\mathrm{k})$ attain values slightly greater than 7.0 in the vicinity of $k=0.75$. But even the average maximum value of 6.0 for the effective negative coefficient of 1 ift indicates that the downward lift forces may attain maximum values 33 percent greater than the maximum possible lift forces acting in the upward direction. Maximum values of $\mathrm{C}_{\mathrm{L}}(\mathrm{k})$ corresponds to a value of $\phi$ of about $45^{\circ}$, which is half way through the phase shift cycle.

The potential flow theory gives a value of $\mathrm{C}_{\mathrm{L}}=4.495$ for zero bottom clearance, with a discontinuous jump to very high negative values of $\mathrm{C}_{\mathrm{L}}$ for a very small clearance (Yamamoto, Nath, and Slotta, 1973). In the potential flow solution, the value of $\mathrm{C}_{\mathrm{L}}$ depends only on the relative clearance; i.e., the ratio clearance-diameter. The coefficient of lift is negative whenever the pipe is not in contact with the bottom, and its magnitude decreases as the relative clearance is increased.

Although the potential flow solution appears to work reasonably well when a pipeline is touching the bottom, this approach does not work when there is a small clearance. This is because viscous effects are very important for the flow through the narrow bottom clearance constriction. The choking phenomenon limits the maximum flow velocities and corresponding pressure drops on the bottom side of the pipeline, thereby limiting the maximum possible downward lift forces.

The results of this investigation indicate that the effective negative coefficient of 1 ift, $C_{L}(k)$, can attain a maximum value of on $1 y$ 7.0. This is much less than the values of $\mathrm{C}_{\mathrm{L}}$ suggested for small relative clearances by potential flow theory. The coefficient of lift is obviously not a simple function of relative clearance, since for a given clearance and diameter, both the lift effect and the coefficient of lift will vary with the waveinduced flow conditions. For the smallest relative clearances, the positive lift forces were larger than the negative lift forces, especially where the horizontal water particle velocities and excursions were high. 


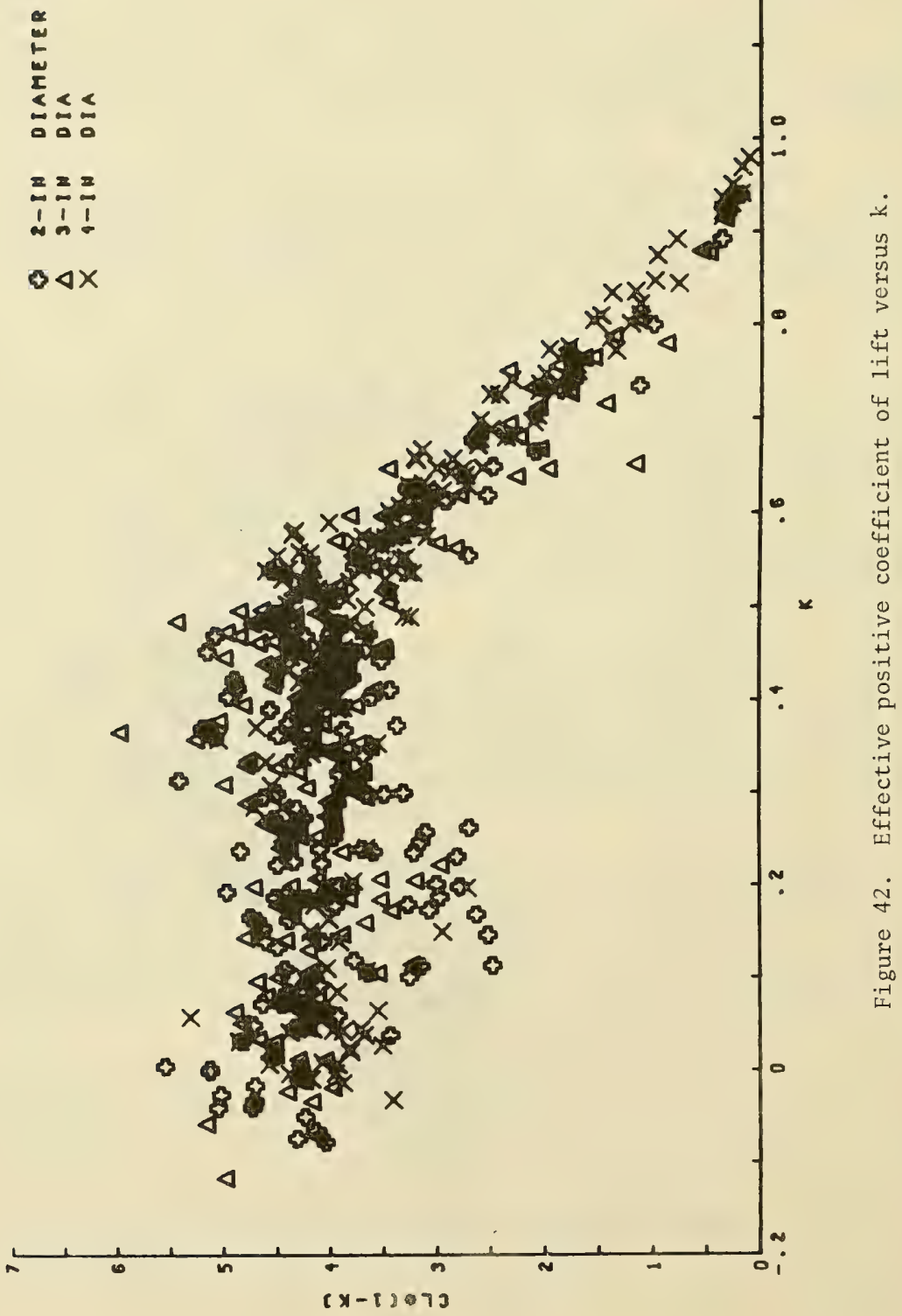




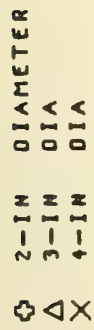

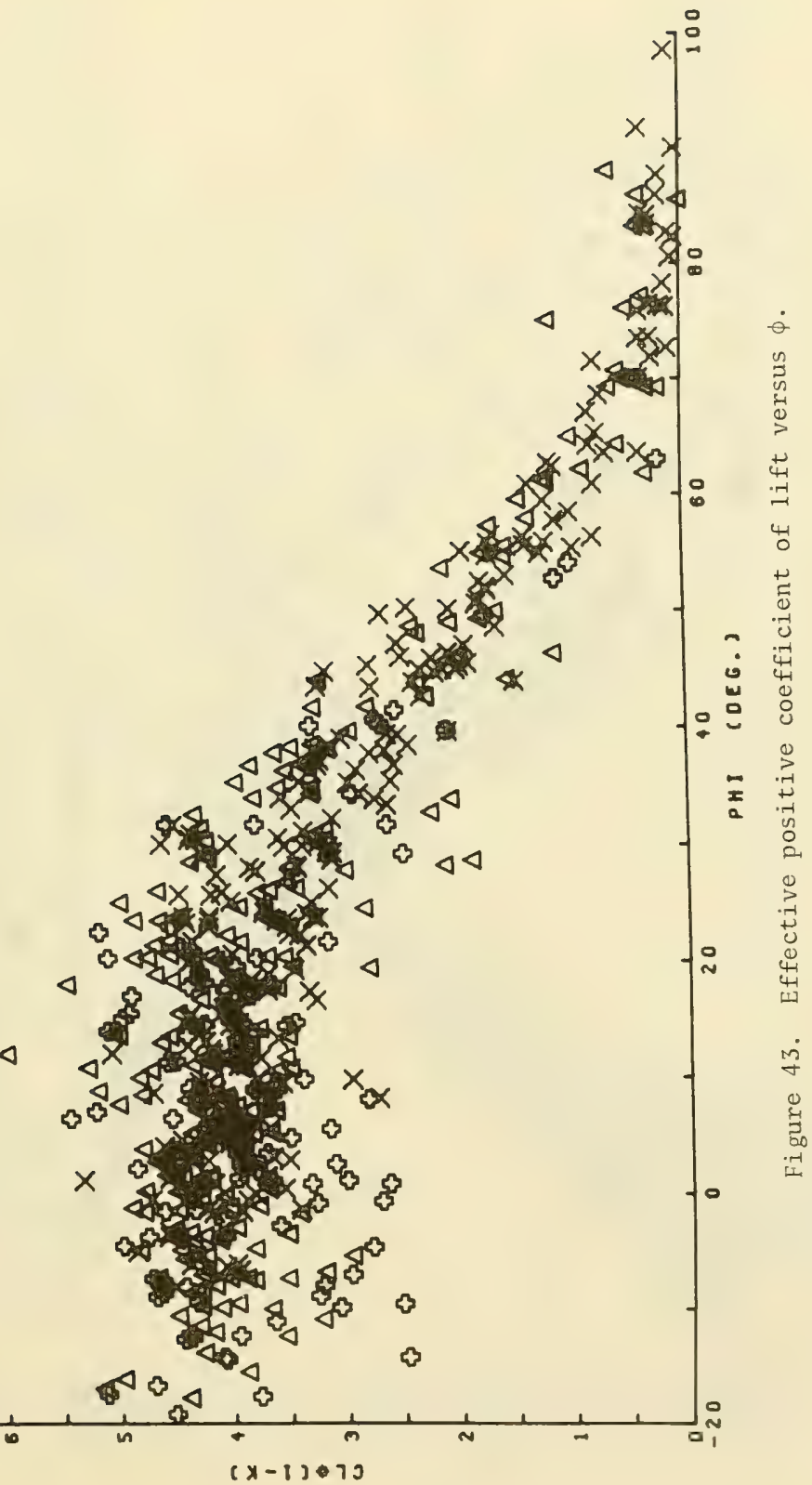




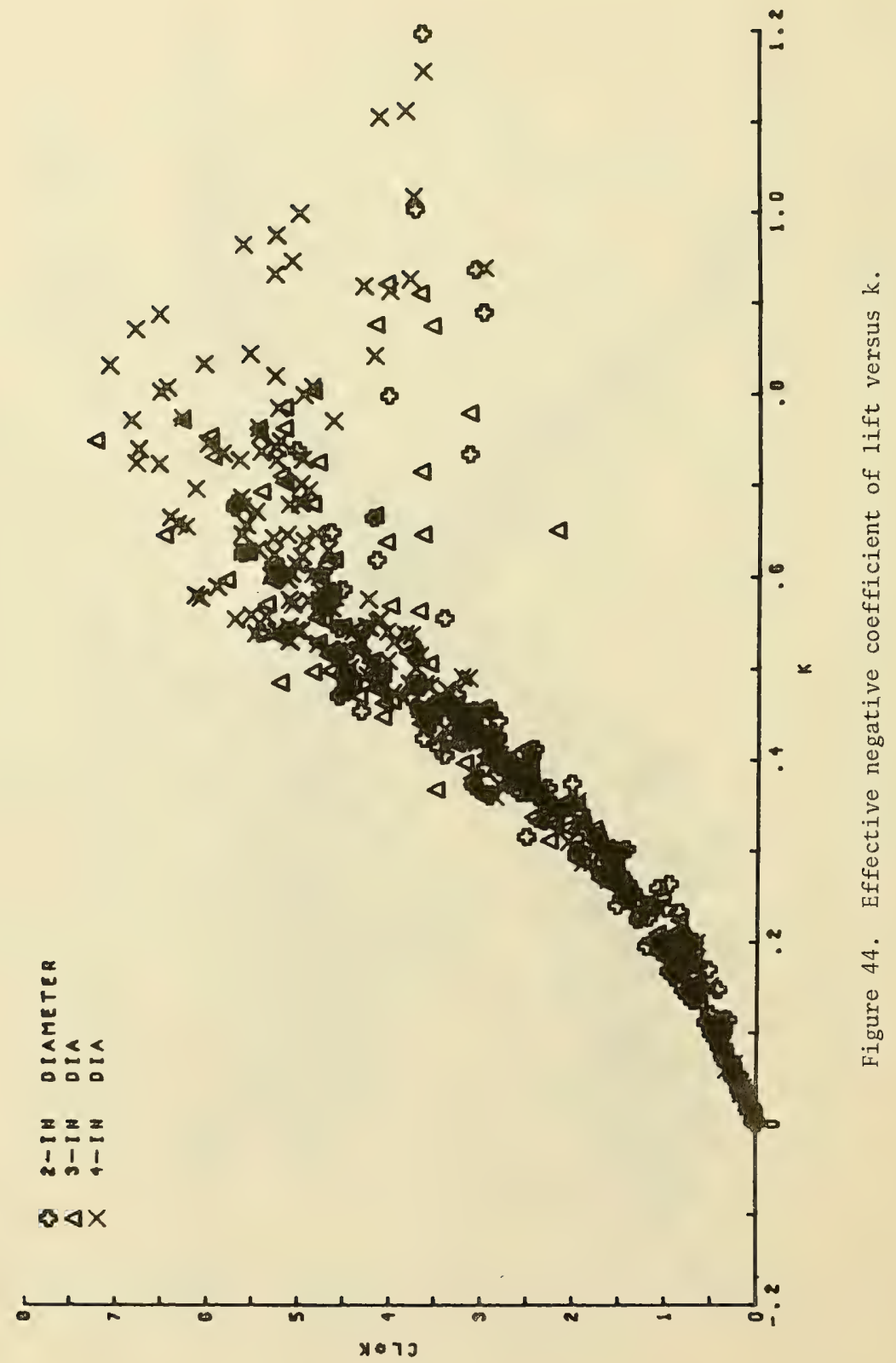




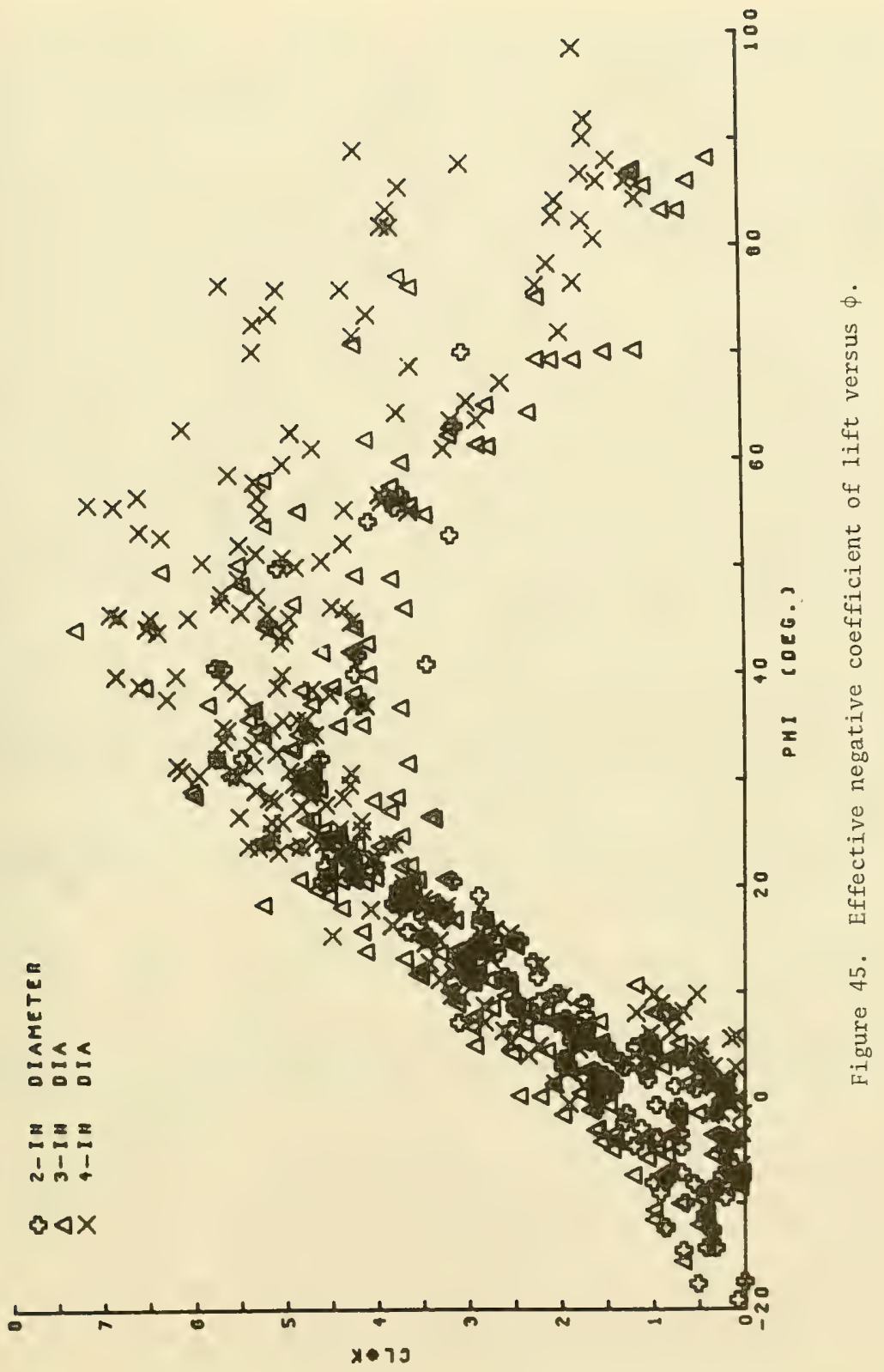


The largest negative 1 ift forces do not occur at clearances where the choking effect is absent (corresponding to $k=1$ and $\phi=90^{\circ}$ ). Rather, the largest values of the effective negative coefficient of lift correspond to values of $\phi=45^{\circ}$ and $\mathrm{k}=0.75$. Interestingly, when $\mathrm{k}=1$ and $\phi=90^{\circ}$ where the positive lift forces have decreased to zero and the choking effect does not develop, the maximum effective negative coefficient of lift is approximately 4.5 , the same magnitude as the potential flow solution for the positive coefficient of lift for zero bottom clearance. However, as the bottom clearance is increased further, $k$ and $\phi$ remain at 1 and $90^{\circ}$, respectively, while the effective negative coefficient of lift decreases to zero (with the diminishing lift forces).

The significance of these results is easily seen by following these relationships for a given pipe and wave as the pipeline is raised from the bottom, and $k$ goes from 0 to 1 . In the interval from $k=0$ to $1 / 2$, the magnitude of the maximum upward lift forces remains the same, which is approximately equal to the potential flow solution for a cylinder in contact with the bottom $\left(\mathrm{C}_{\mathrm{L}}=4.5\right)$. However, at the same time, the negative lift forces increase continuously, reaching a magnitude equal to the positive lift forces at $\mathrm{k}=1 / 2\left(\mathrm{C}_{\mathrm{L}}(1-\mathrm{k})=\mathrm{C}_{\mathrm{L}}(\mathrm{k})=4.5\right)$. Simultaneously, there is a shift in the positions of both the maximum positive and negative lift forces, since $\phi$ increases from $0^{\circ}$ to $30^{\circ}$.

In the interval $\mathrm{k}=1 / 2$ to ?, the maximum positive lift forces continuously decrease to zero. At the same time, the maximum negative lift forces increase to reach a maximum value at $\mathrm{k}=0.75$ (where $\mathrm{C}_{\mathrm{L}}(\mathrm{k})=$ 6. or 7.), and then decrease back to a maximum corresponding to $\mathrm{C}_{\mathrm{L}}(\mathrm{k})=$ 4.5 at $k=1$. The point of maximum negative 1 ift corresponds to $\phi=45^{\circ}$, the midpoint of the phase shift cycle.

The phase shift of the maximum lift forces is only half as much in the interval $\mathrm{k}=0$ to $1 / 2$ (where $\phi$ goes from $0^{\circ}$ to $30^{\circ}$ ) as in the interval $k=1 / 2$ to 1 (where $\phi$ goes from $30^{\circ}$ to $90^{\circ}$ ).

At $k=1$, only negative 1 ift forces exist, and these go to zero as the bottom clearance is increased further.

Al1 of the above interrelationships between $\phi, k, C_{L}, C_{L}(1-k)$, and $\mathrm{C}_{\mathrm{L}}(\mathrm{k})$ were the same for all pipe diameters tested, regardless of the angle of orientation (provided that $\mathrm{C}_{\mathrm{L}}$ was calculated considering only the component of the horizontal velocity perpendicular to the pipeline axis). Thus, for the range of conditions tested, these interrelationships were independent of the scale and configuration of the pipeline. Also, there is no mention of the wave conditions, which indicates the interrelationships are independent of the wave conditions as well.

The relationships between the parameters, $C_{L}, \phi$, and $k$, defining the lift force equation are useful, since if either $\phi$ or $k$ is known, the other two parameters can be determined. All that is needed is a relationship between $\phi$ or $k$ and the wave and pipeline conditions. 
There appears to be a better correlation between $\mathrm{k}$ and the parameters involving $\mathrm{C}_{\mathrm{L}}\left(\mathrm{C}_{\mathrm{L}}, \mathrm{C}_{\mathrm{L}}(1-\mathrm{k})\right.$, and $\left.\mathrm{C}_{\mathrm{L}}(\mathrm{k})\right)$ than between the analogous relationships using $\phi$, so the former relationships should be used. Also, in comparing the plots of $C_{L}(1-k)$ versus $k$ (Fig. 42) and $C_{L}(k)$ versus $k$ (Fig. 44), the scatter appears minimal in the plot with $\mathrm{C}_{\mathrm{L}}(\mathrm{k})$ for the interval of $k$ between 0 and $1 / 2$. For the interval of $k$ between $1 / 2$ and 1 , the scatter is much less on the plot between $C_{L}(1-k)$ and $k$. Therefore, it is suggested that when determining a value of $\mathrm{C}_{\mathrm{L}}$ for a given value of $\mathrm{k}$, the plot of $C_{L}(k)$ versus $k$ be used for values of $k$ less than $1 / 2$ (except for $k$ close to 0$)$, and the plot of $C_{L}(1-k)$ versus $k$ be used for values of $k$ greater than $1 / 2$ (except for $k$ close to 1) (see Fig. 46). For $k$ close to 0 , it can be assumed that $C_{L}=4.5$. However, for $k \approx 1$, the value of $C_{L}$ can vary from about 4.5 to zero, since as the clearance is increased from the point where $\phi=90^{\circ}$ and $k=1$, both $\phi$ and $k$ remain at their maximum values of $90^{\circ}$ and 1 , respectively, while the lift effect diminishes to zero.

When the above relationships between $\phi, k, C_{L}, C_{L}(1-k)$, and $C_{L}(k)$ are plotted for only the 4-inch-diameter pipe model, the scatter is reduced. Although the data for all three diameters completely overlap (showing the same relationships hold for all diameters), the amount of scatter increases with the smaller diameter models. This is because the data extend to higher relative clearances (clearance-diameter) for the smaller diameter models than the corresponding data for the 4-inch-diameter model, since all models were tested at the same actual clearances.

Since the lift effect diminishes at high values of the relative clearance, the lift forces on the snaller diameter models at the largest bottom clearances were very small in many cases. This is especially true for the smaller waves and higher orientation angles, where the horizontal velocities perpendicular to the pipeline were very low. In such cases, the lift forces were often insignificant, so the values of $\mathrm{C}_{\mathrm{L}}$, $\phi$, and $\mathrm{k}$ calculated from the least squares analysis were not as accurate.

In addition, as the lift forces decrease with high relative clearances, eddy-induced forces may approach the magnitude of the lift forces, thus introducing further error in the calculated values of $\mathrm{C}_{\mathrm{L}}, \phi$, and $\mathrm{k}$.

The lift forces were generally significant for all clearances tested using the 4-inch-diameter pipe section, and since the measured forces were larger, the experimental error involved in measuring them was less than for the smaller diameter models.

Because of this, the data taken for very large bottom clearances were not included in the plotted relationships. For higher clearances, values of $\mathrm{k}$ and $\phi$ equal to 1 and $90^{\circ}$, respectively, would be expected, since the choking phenomenon would not occur throughout the vave cycle. However, as the clearance is increased, the lift effect diminishes, resulting in decreasing values of the coefficient of lift. 


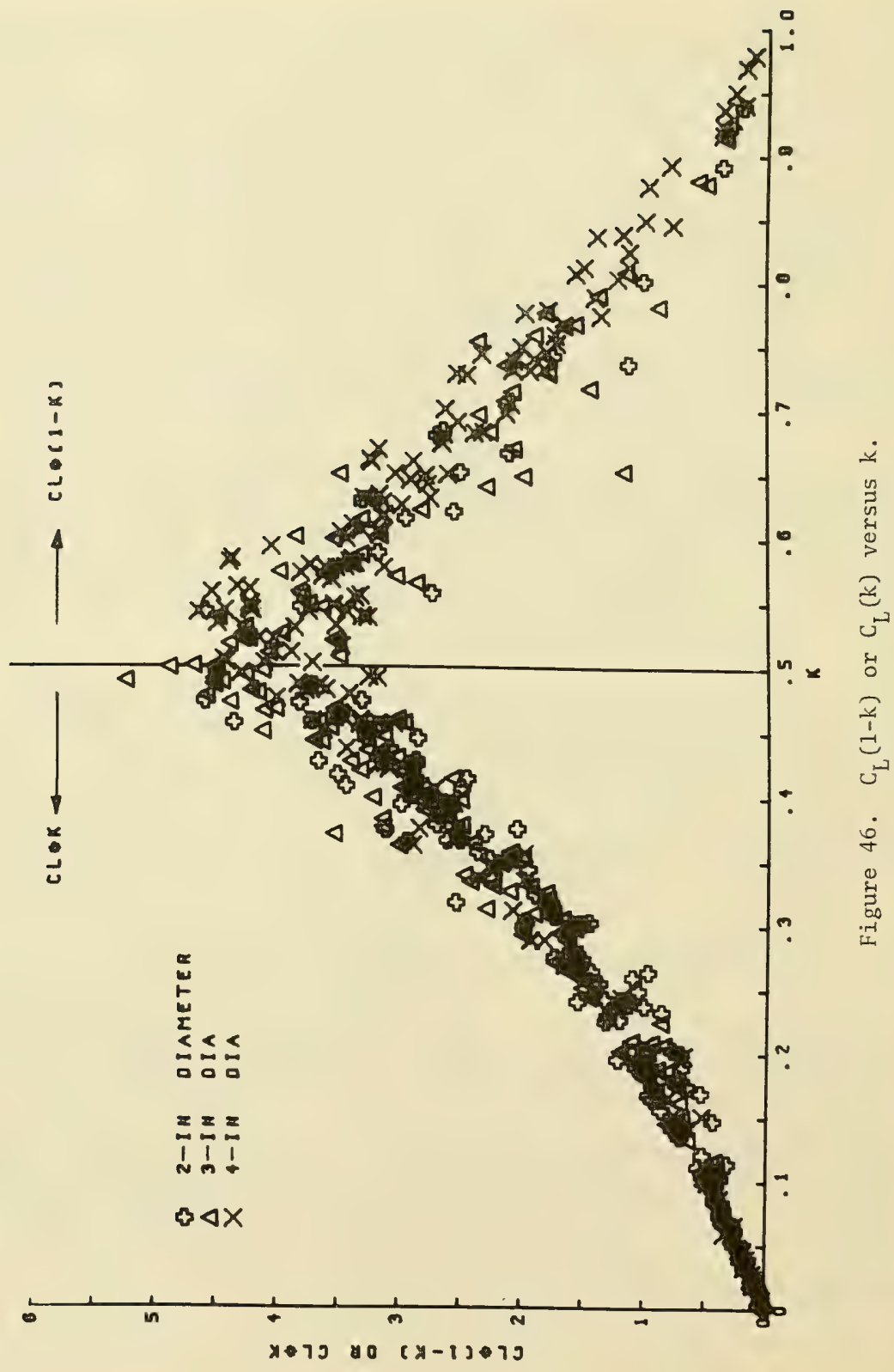


If such data were included in the plots of $\mathrm{C}_{\mathrm{L}}$ versus $\mathrm{k}$ and $\phi$, values of $\mathrm{C}_{\mathrm{L}}$ ranging from 0 to the maximum values shown in Figures 40 and 41 would be present in the vicinity of $\mathrm{k}=1$ and $\phi=90^{\circ}$ in the respective plots. The same applies to the plots of $\mathrm{C}_{\mathrm{L}}(\mathrm{k})$ versus $\mathrm{k}$ and $\phi$.

These trends were observed in the data taken for the largest bottom clearances ( 1 and 2 inches). However, since these lift forces were so smal1, a significant amount of error could be introduced into the calculated values of $\mathrm{C}_{\mathrm{L}}, \phi$, and $\mathrm{k}$ because of the presence of eddy-induced forces, as discussed above. Therefore, these data were omitted from the plotted relationships, since errors in $\phi$ or $k$ corresponding to low values of $\mathrm{C}_{\mathrm{L}}$ would produce considerable scatter, obscuring the valid relationships showr.

4. Relationships Between $\phi$ and $\mathrm{k}$ and Parameters Defining the Wave and Pipeline Conditions.

To use the above relationships between $C_{L}, \phi$, and $k$ to determine the wave-induced lift forces acting on a pipeline, either $\phi$ or $k$ must be known. Thus, a value of one of these parameters must be determined from relationships of $\phi$ or $k$ with the wave conditions and pipeline configuration.

The lift force phenomenon is a function of the following variables:

(a) Pipeline configuration

(1) Diameter

(2) Clearance

(3) Orientation angle

(b) Fluid properties

(1) Density

(2) Viscosity

(c) Wave-induced flow conditions

(1) Maximum horizontal water particle velocity perpendicular to the pipeline axis

(2) Wave period, which represents the duration of the flow in one direction

(3) Length of the horizontal excursions of the water particles perpendicular to the pipeline axis (this quantity is directly proportional to the product of the above two parameters)

Assuming that only water with a limited range of temperature is being dealt with, the fluid properties will be ignored for the present. The orientation angle of the pipeline can be handled as discussed above, considering only the components of the horizontal fluid motions 
perpendicular to the pipeline axis. Since the length of the horizontal water particle excursions is directly proportional to the product of the wave period and the maximum horizontal water particle velocity, only four independent variables are left: diameter, clearance, horizontal water particle velocity, and wave period. Thus, any single parameter used to relate $\mathrm{C}_{\mathrm{L}}$, $\phi$, or $\mathrm{k}$ to the wave and pipeline conditions must include these four variables. This constraint is necessary if the relationship is expected to be valid for general application under any set of wave and pipeline conditions.

The four variables can be arranged into several dimensionless parameters. The important parameters should include the following:

(1) relative clearance, clear/Dia

where clear = bottom clearance

Dia = pipe diameter

(2) Keulegan-Carpenter parameter, $u_{\max } \mathrm{T} / \mathrm{Dia}$

$$
\text { where } \begin{aligned}
\mathrm{T}= & \text { wave period } \\
\mathrm{u}_{\max }= & \text { component of maximum horizontal water particle } \\
& \text { velocity perpendicular to the pipeline axis }
\end{aligned}
$$

(3) $c 1$ ear $/ u_{\max } \mathrm{T}$

NOTE.--Not all of these parameters are necessary to describe the system since some are redundant, but some may be more useful than others.

Since viscosity is an important variable involved in the choking phenomenon, the Reynolds number, $u_{\max } \mathrm{Dia} / \nu$, and a Reynolds number for the clearance, $u_{\max } c l e a r / v$, are also important parameters (where $v=$ kinematic viscosity).

The dimensionless parameters, clear/ $u_{\max } \mathrm{T}, \mathrm{u}_{\max } \mathrm{T} / \mathrm{Dia}, \mathrm{u}_{\max } \mathrm{clear} / \mathrm{\nu}$, and $u_{\max } \mathrm{Dia} / \nu$, were plotted versus the lift force parameters, $C_{L}, \phi, k$, $\mathrm{C}_{\mathrm{L}}(1-\mathrm{k})$, and $\mathrm{C}_{\mathrm{L}}(\mathrm{k})$, for constant values of the relative clearance, clear/Dia. The correlation was not good with the parameters involving the coefficient of 1 ift $\left(C_{L}, C_{L}(1-k)\right.$, and $\left.C_{L}(k)\right)$. However, good correlation was found between several of the dimensionless parameters and the quantities $\phi$ and $k$.

The parameter, clear/u $\mathrm{max}$, exhibited the best correlation with both $\phi$ and $\mathrm{k}$ for each relative clearance, although there was some variation in these relationships for the data corresponding to the different pipe diameters (see Figs. 47 to 52). Although the differences are not large, the data do indicate the presence, of a scale effect in these relationships. 


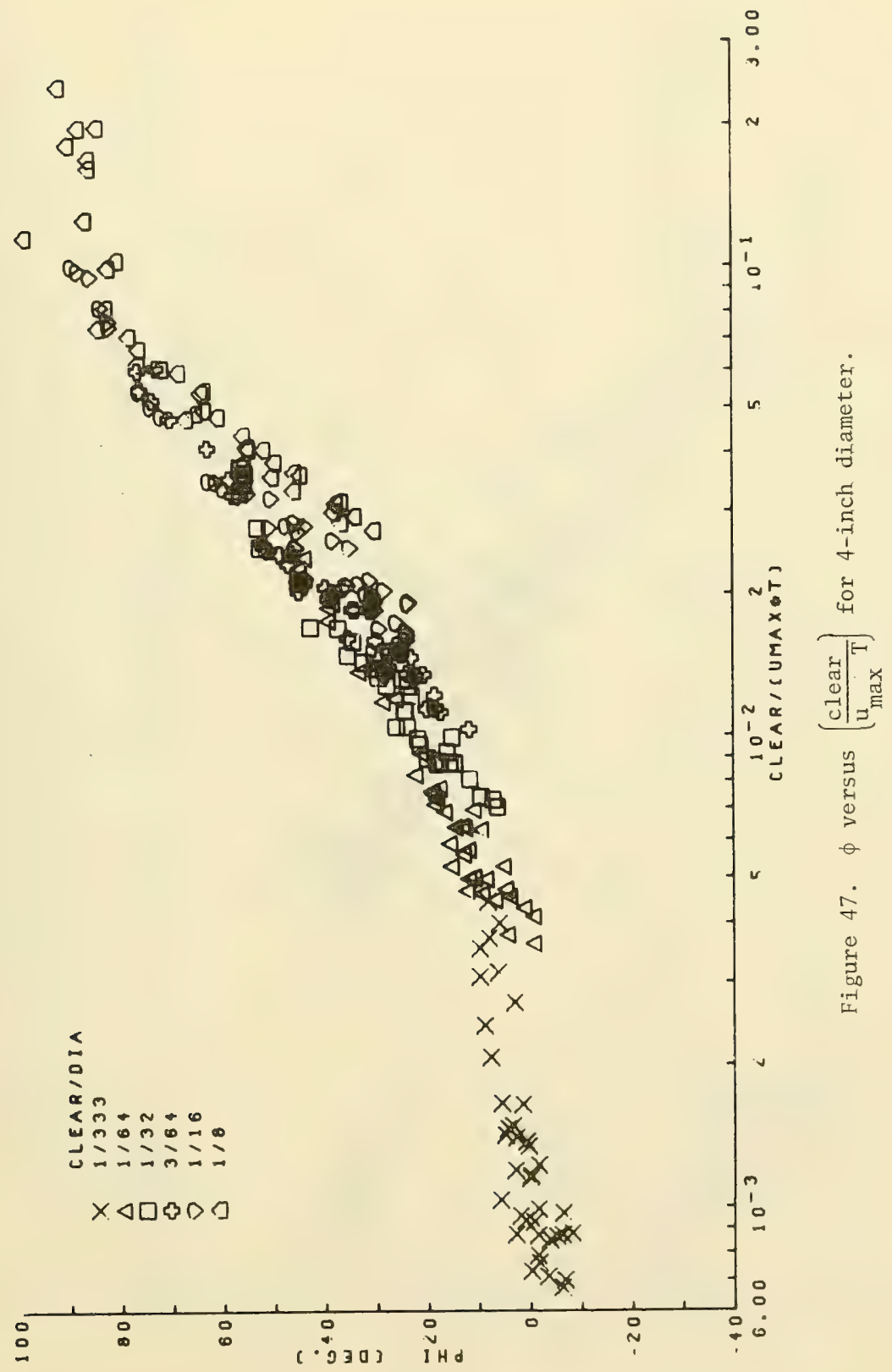




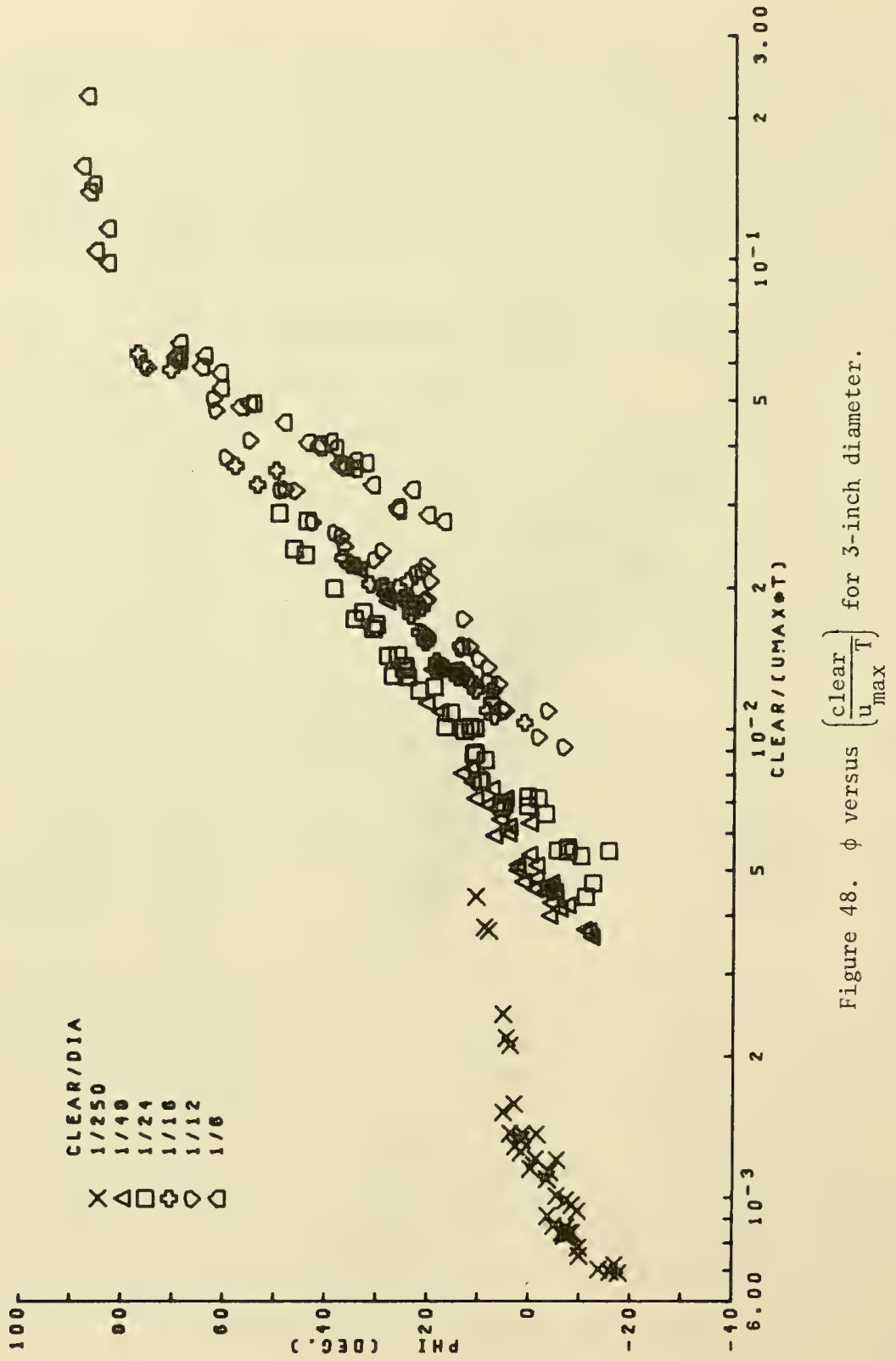




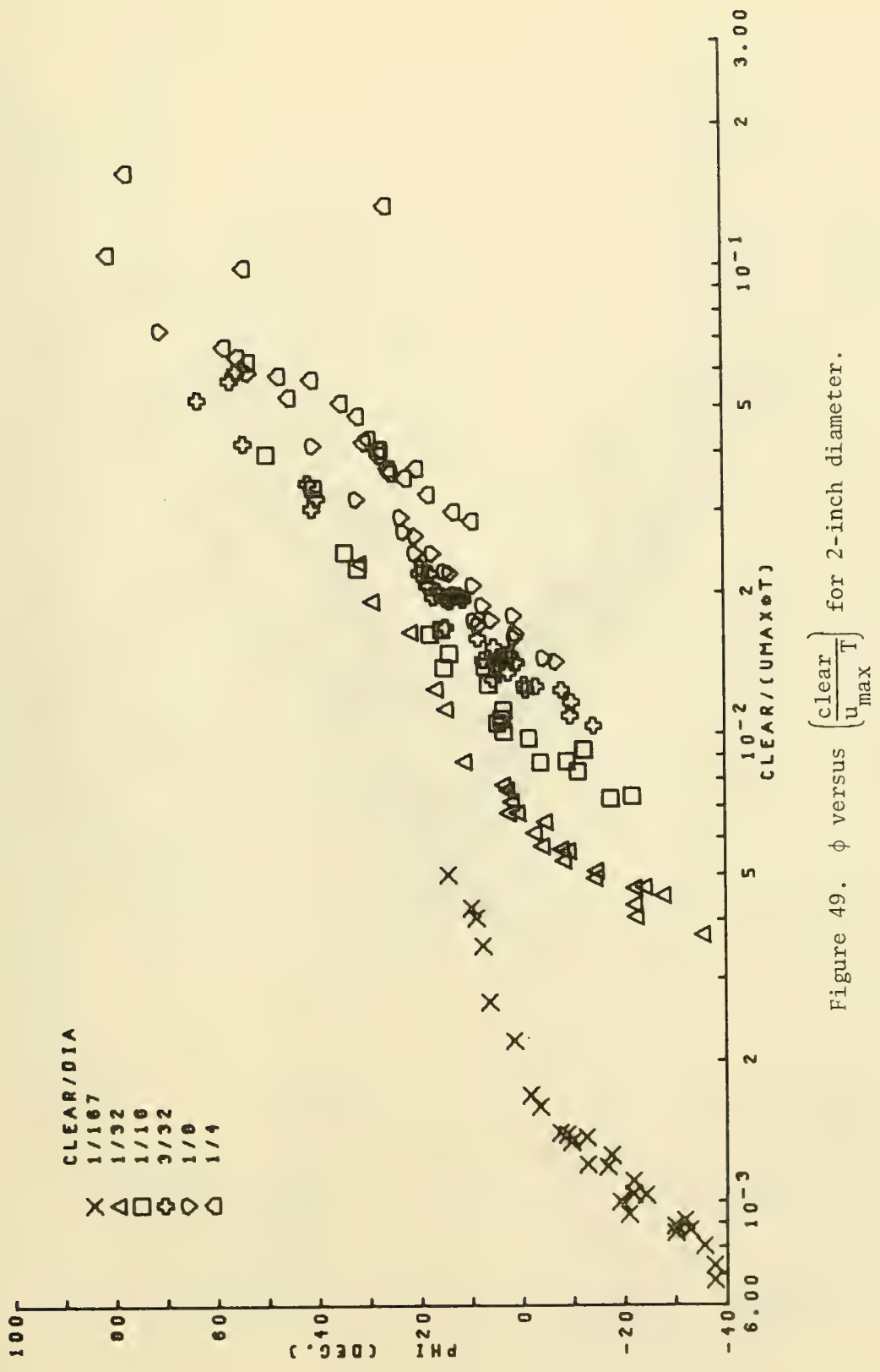




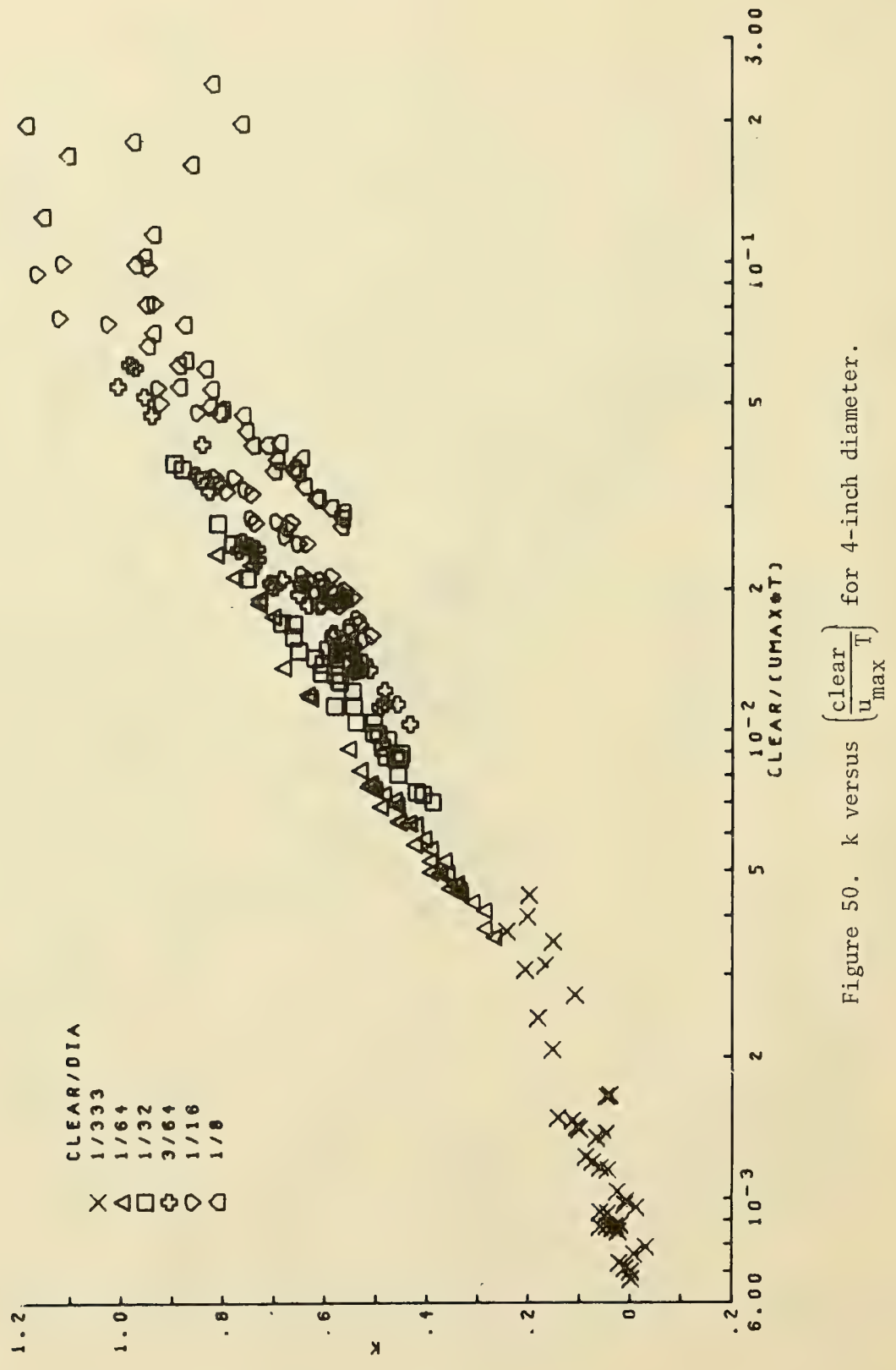




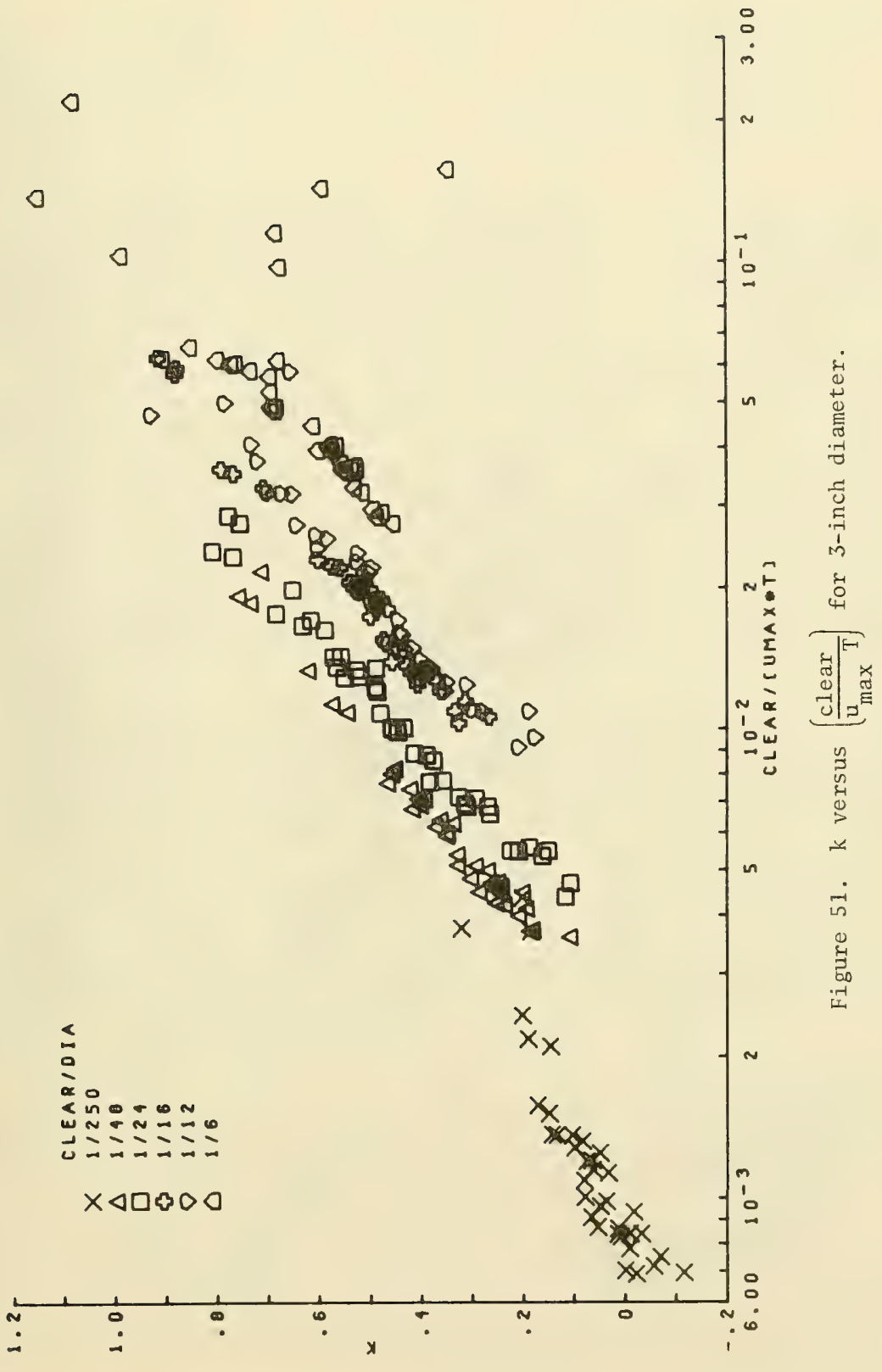




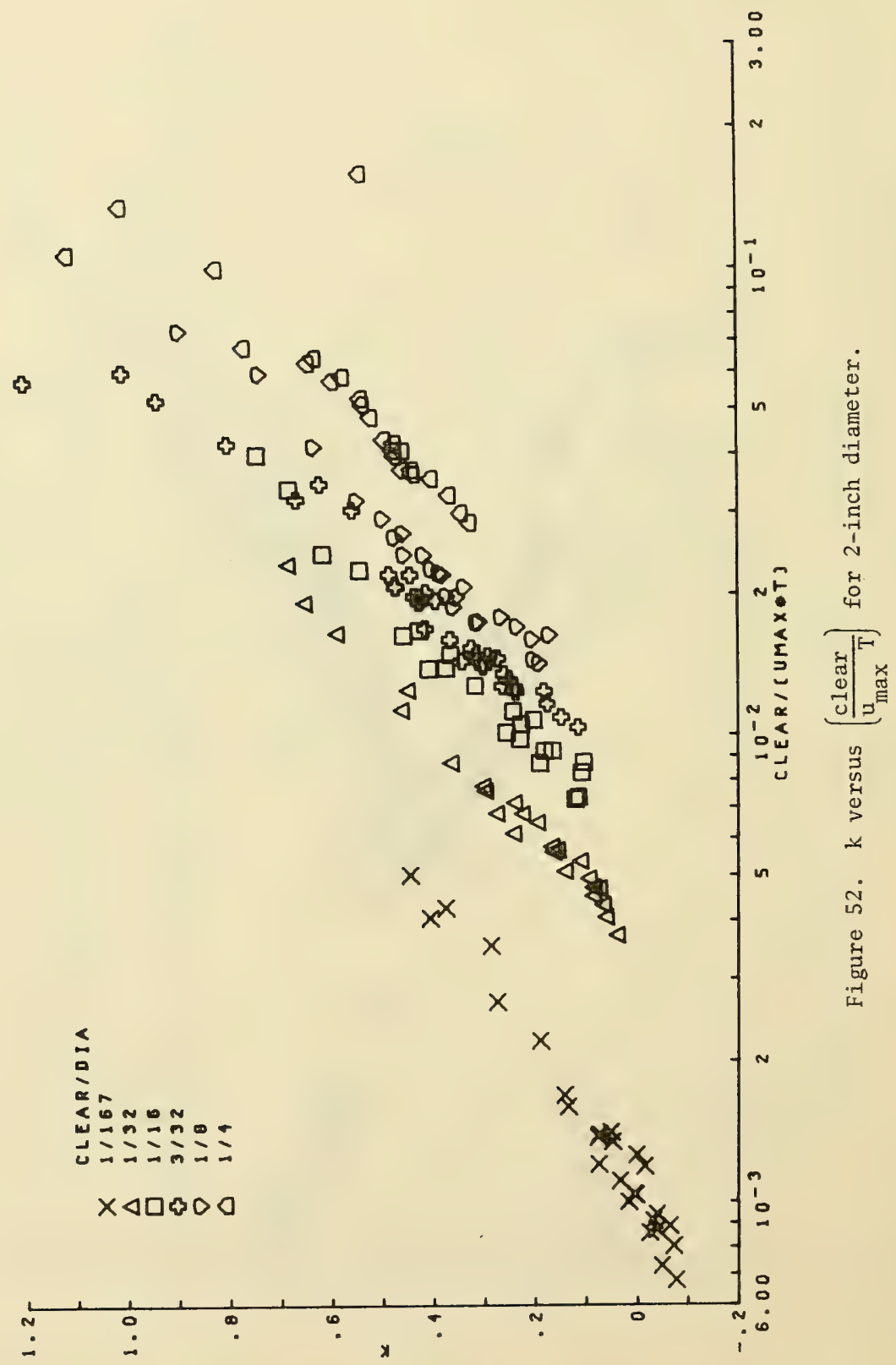


$\phi$ and $\mathrm{k}$ were also correlated with the Keulegan-Caxpenter parameter, $u_{\max } \mathrm{T} / \mathrm{Dia}$. However, these relationships were not the same when the data corresponding to a given relative clearance were compared for different pipe diameters. The relationships were the same for a given absolute clearance, rather than a relative clearance (clear/Dia). These relationships are shown in Figures 53 and 54 for the combined data from all three pipe diameters.

The parameter, $u_{\max } c l e a r / \nu$, demonstrated correlation with both $\phi$ and $k$, but these relationships also exhibited a scale effect, such that the relationships for a given relative clearance were not the same when comparing the data for different pipe diameters. Figures 55 and 56 are examples of these relationships for the 4-inch-diameter pipeline.

Correlation between the Reynolds number, $u_{\max } \mathrm{Dia} / \nu$, and the parameters, $\phi$ and $k$, was not good, especially when comparing the data for the different pipe diameters.

Since none of the above dimensionless parameters alone could be used to determine a value of $\phi$ or $\mathrm{k}$ for any given pipe diameter, clearance, and wave condition due to the presence of scale effects, several. of the parameters were combined in various ways to form different dimensionless parameters containing all four of the important variables (clear, Dia, $u_{\max }$, and $\mathrm{T}$ ). An attempt was made to find a single parameter containing all of the important variables that was well correlated with $\phi$ or $k$ for all wave conditions, pipeline sizes, and configurations.

Several relationships were found that exhibited good correlation for all the wave and pipeline conditions tested. However, since this is a model study and, therefore, limited to lower values of the KeuleganCarpenter parameter and Reynolds number than prototype design situations in the ocean, caution should be used in extrapolating these results.

The dimensionless combination, (clear/umax $\mathrm{T})\left(\mathrm{Dia} / \mathrm{u}_{\max } \mathrm{T}\right)$, demonstrated the best correlation with both $\phi$ and $k$ for all conditions tested. These relationships are given in Figures 57 and 58 . Since both $\mathrm{k}$ and $\phi$ define the point at which choking occurs in the wave cycle, it appears that the choking phenomenon is directly dependent on the water particle excursions relative to both the pipe diameter, (Dia/umax $T$ ), and the bottom clearance, $\left(c l e a r / u_{\max } \mathrm{T}\right)$.

Although the parameter, (clear/u max $T)$, is equivalent to the ratio of the bottom clearance to the horizontal excursion of the water particles (differing only by the constant $1 / \pi$ ), the quantity ( $u_{\max } \mathrm{T}$ ) should not be thought of only as defining the length of the water particle excursions. Both variables, $u_{\max }$ and $\mathrm{T}$, are independently important in defining the choking phenomenon. The larger $u_{\max }$, the sooner the choking conditions will develop in the wave cycle for a given clearance and pipe diameter. Similarly, since the wave period, $T$, defines the duration of the horizontal flow in one direction, the larger the wave period, the sooner choking will develop relative to the temporal length of the wave cycle. 

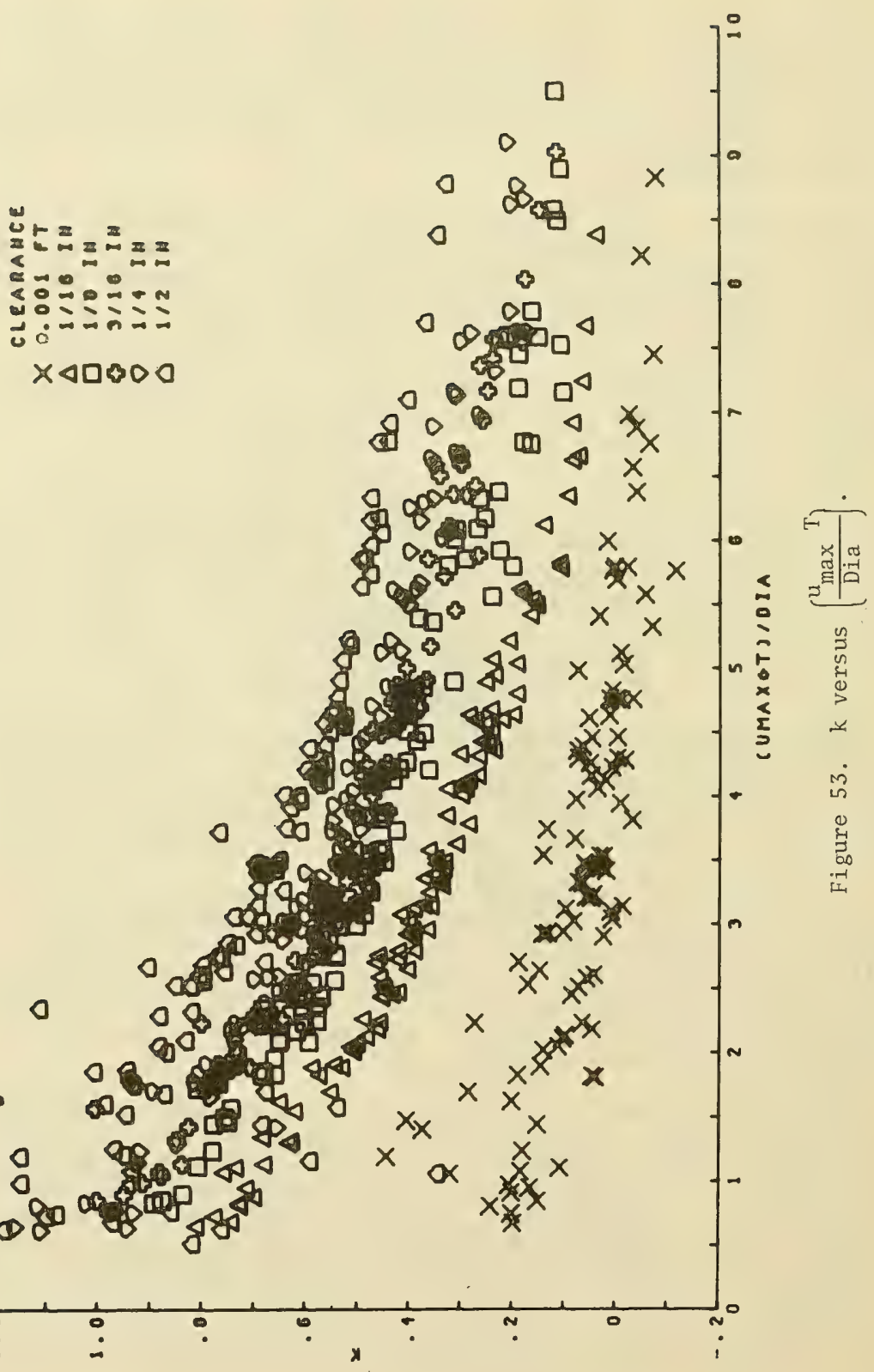


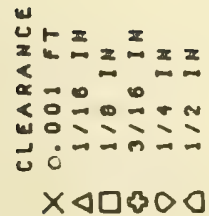

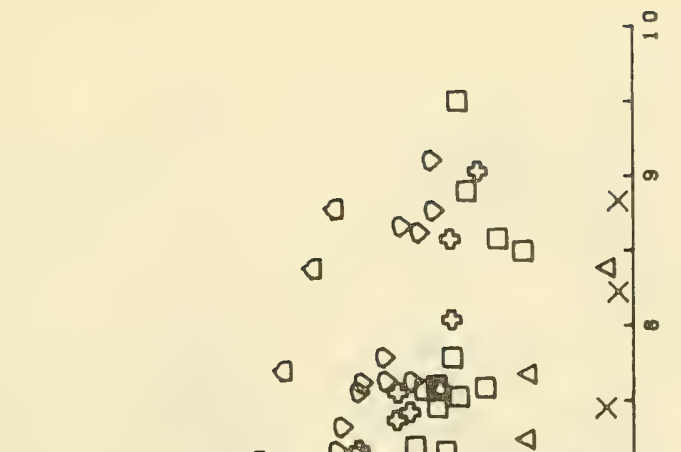

.

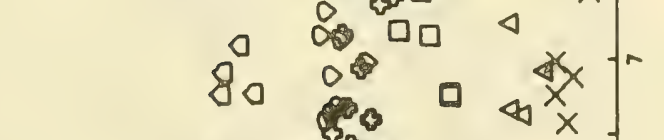

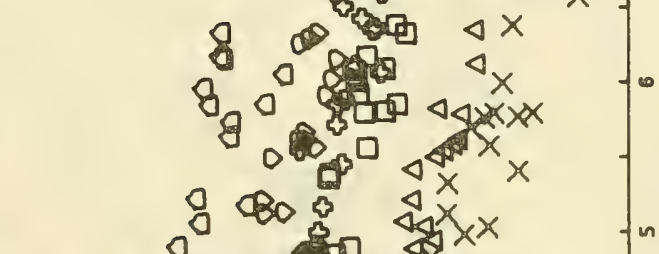

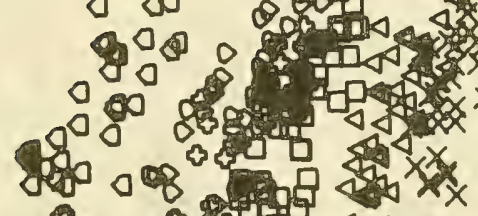

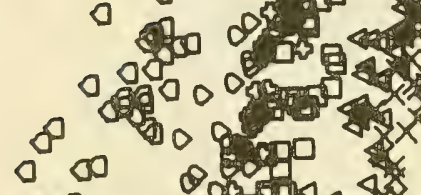

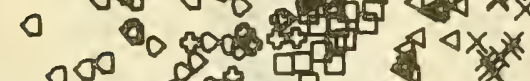

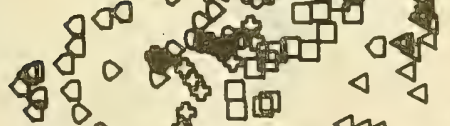

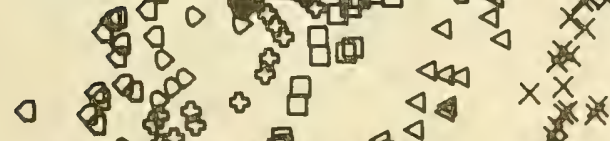

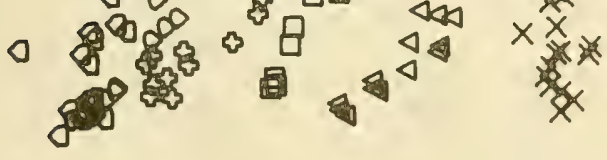




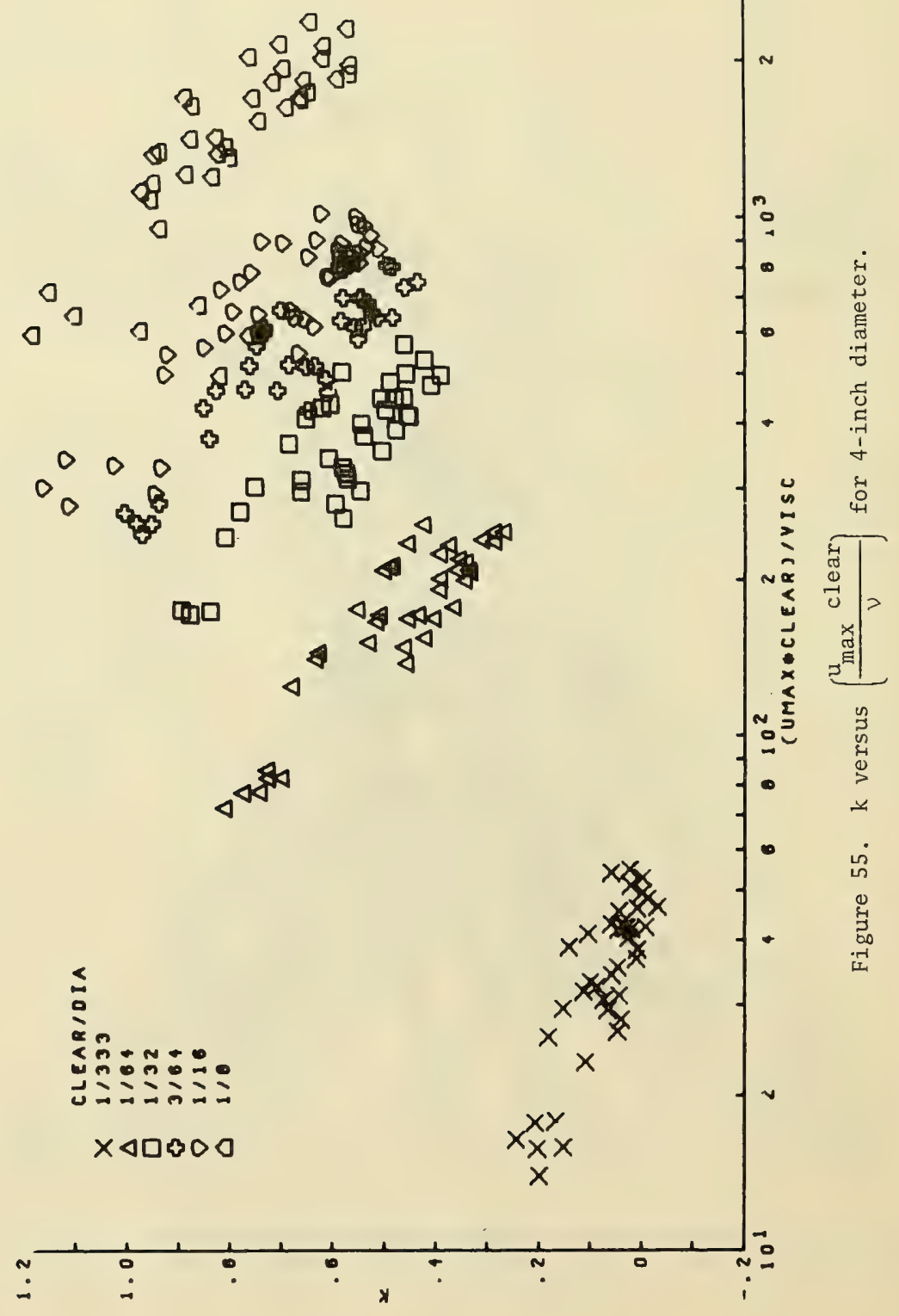




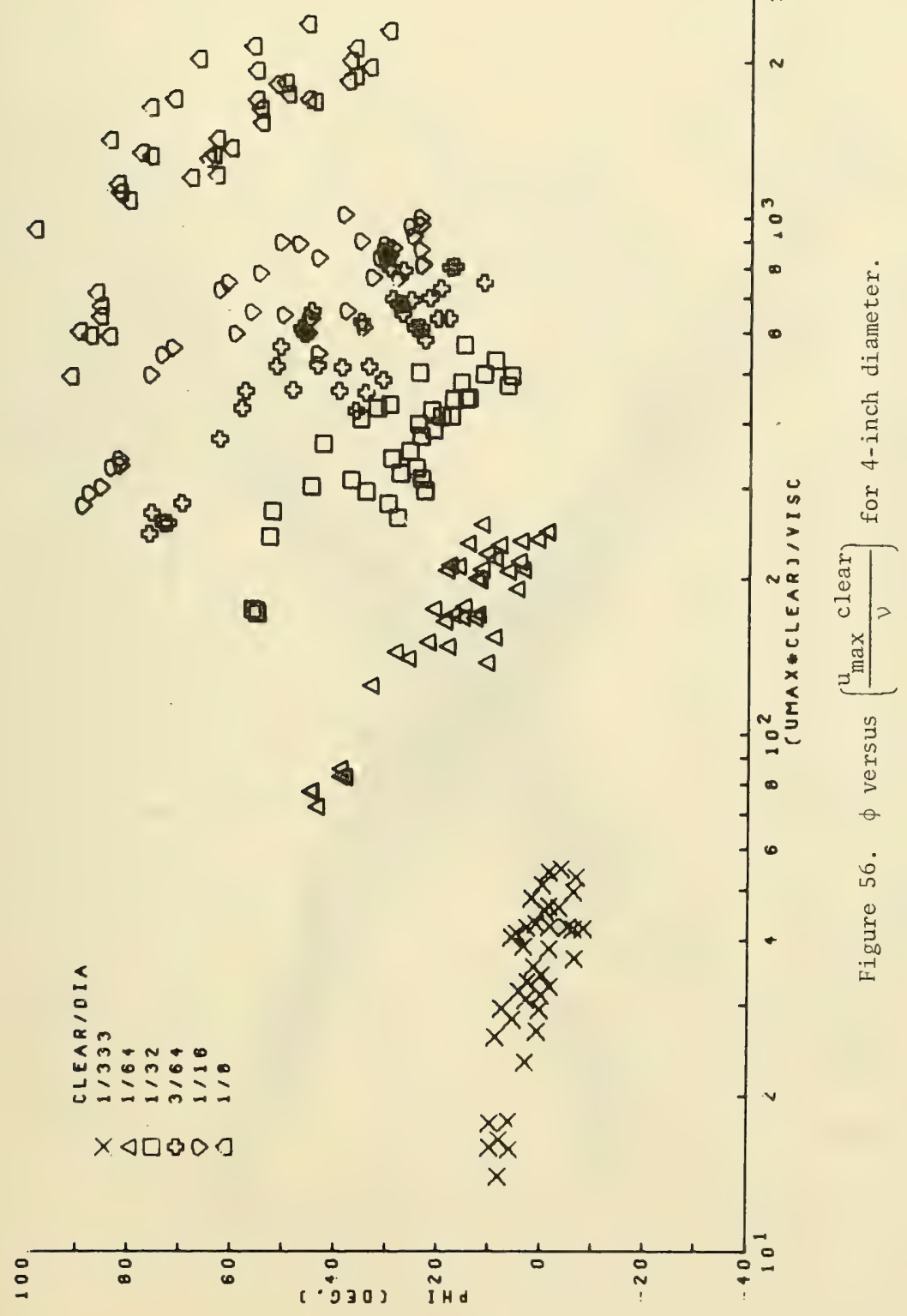




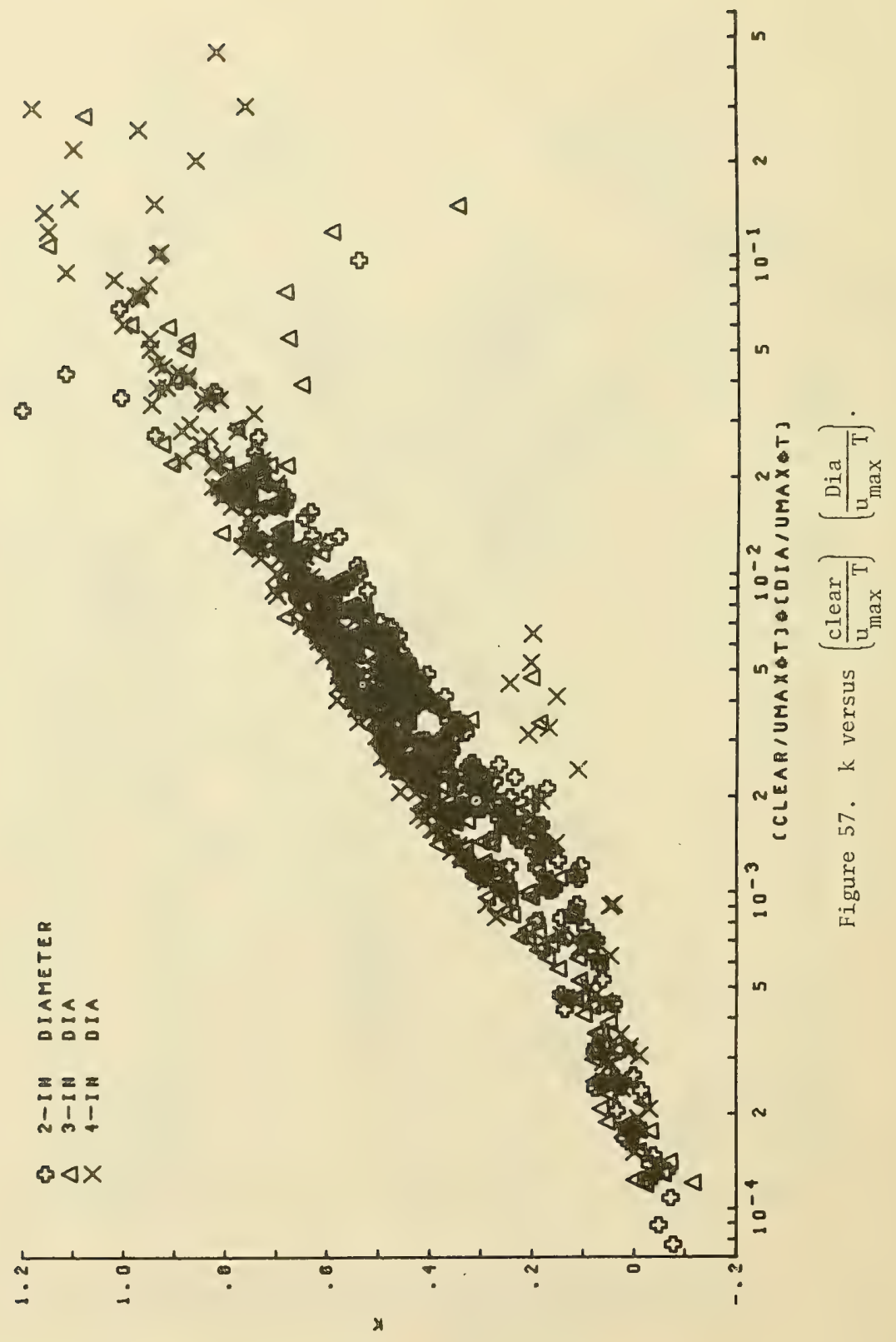




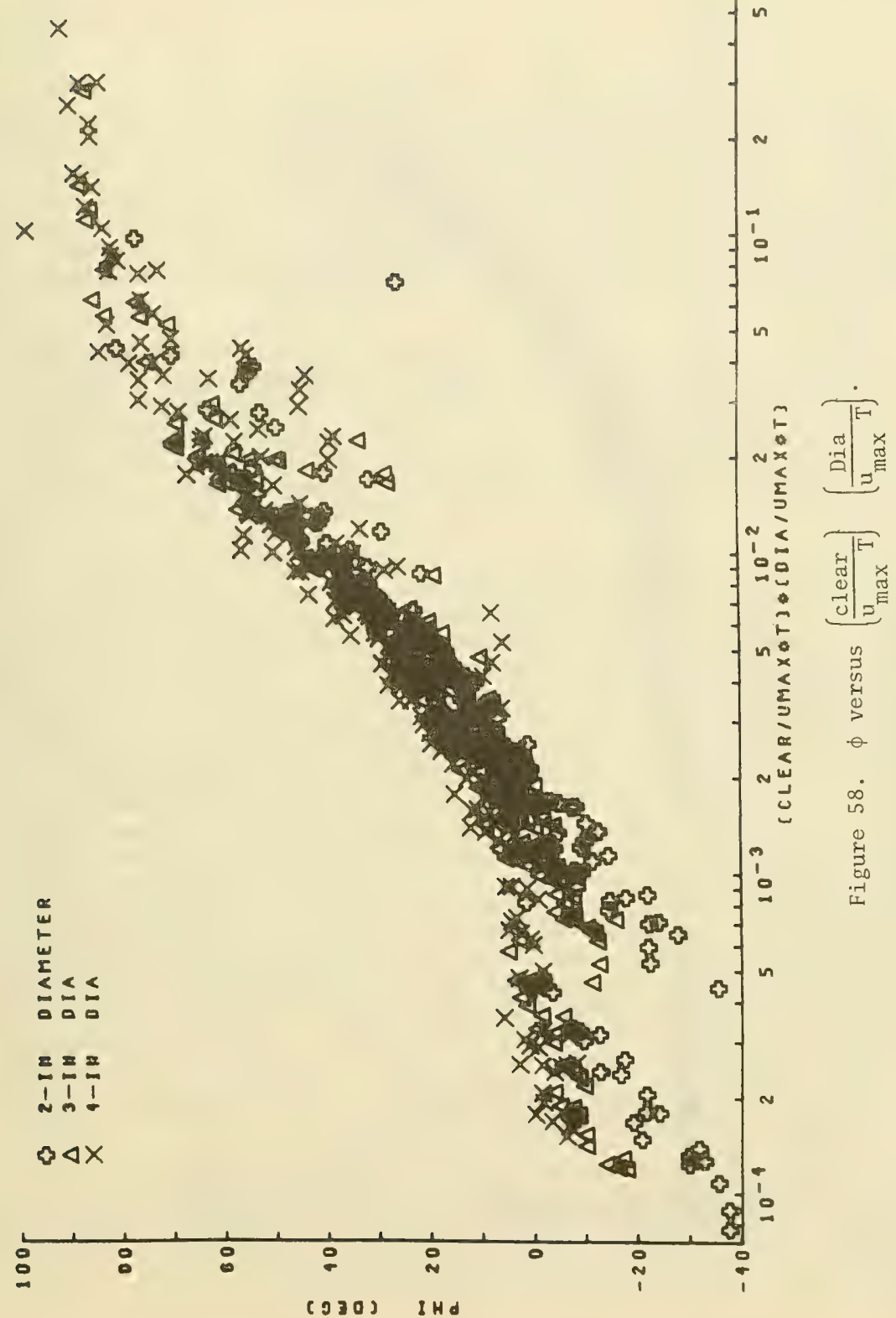


The slight amount of scatter in these plots in the vicinity of $\mathrm{k}=$ 1 and $\phi=90^{\circ}$ is due to the error in calculated values of $\phi$ and $\mathrm{k}$ for the largest bottom clearances where the 1 ift effect was small (as discussed above).

Larger values of the dimensionless combination, (clear/ $\left.u_{\max } \mathrm{T}\right)$ $\left(D i a / u_{\max } T\right)$, than given in the plots would correspond to larger bottom clearances and pipe diameters relative to the maximum velocities, wave periods, and water particle excursions. For these conditions, the values of $k$ and $\phi$ would remain at 1 and $90^{\circ}$, respectively, while the lift effect would eventually diminish to zero with increasing values of this parameter. These trends are evident in the data taken at the largest bottom clearances ( 1 and 2 inches), although these data were not included in the above plots.

Similarly, lower values of the dimensionless parameter than given in the plots would correspond to higher maximum velocities, wave periods, and water particle excursions relative to the smallest bottom clearances and pipe diameters. So for lower values of this parameter, both $k$ and $\phi$ should remain at their defined minimum values of 0 and $0^{\circ}$, respectively, corresponding to lift forces acting in the upward direction only, with very little or no flow possible under the pipe section.

Although $\phi$ was defined as varying from $0^{\circ}$ to $90^{\circ}$ only, negative values of $\phi$ are exhibited in the data for the lowest values of the dimensionless parameters plotted. However, since most of these data points correspond to the smallest diameter pipeline model tested ( 2 inches), this could be partly due to experimental error, since the measured forces were smallest for the smallest model. Also, part of this discrepancy could be due to the difficulty of accurately defining the peak of the wave crest in the experimental wave records. This point was arbitrarily defined as the midpoint of the zero crossings on either side of the wave crest in the digitized data records. However, in some cases, the waves were not perfectly symmetrical, so the maximum elevation of the water surface did not coincide exactly with the midpoint of the zero crossings. This was especially true of the largest waves with the longest periods, which in the plotted relationships would correspond to the minimum values of the dimensionless parameters (at the lowest bottom clearance tested). Thus, the actual kinematics under these waves would be slightly out of phase with the calculated kinematics, resulting in an error in the calculated value of $\phi$. However, this source of error should be the same for the large-diameter models as for the smallest models.

5. Relationships Between $\phi$ (clear/Dia) and k (clear/Dia) and Parameters Defining the Wave and Pipeline Conditions.

Many other useful relationships were found by multiplying $\phi$ and $\mathrm{k}$ by the relative clearance, (clear/Dia), and plotting these dimensionless products versus various dimensionless parameters defining the wave and pipe conditions. Figures 59 to 62 are examples, although several other parameters also showed good correlation with $\phi$ (clear/Dia) and $k$ (clear) Dia). 


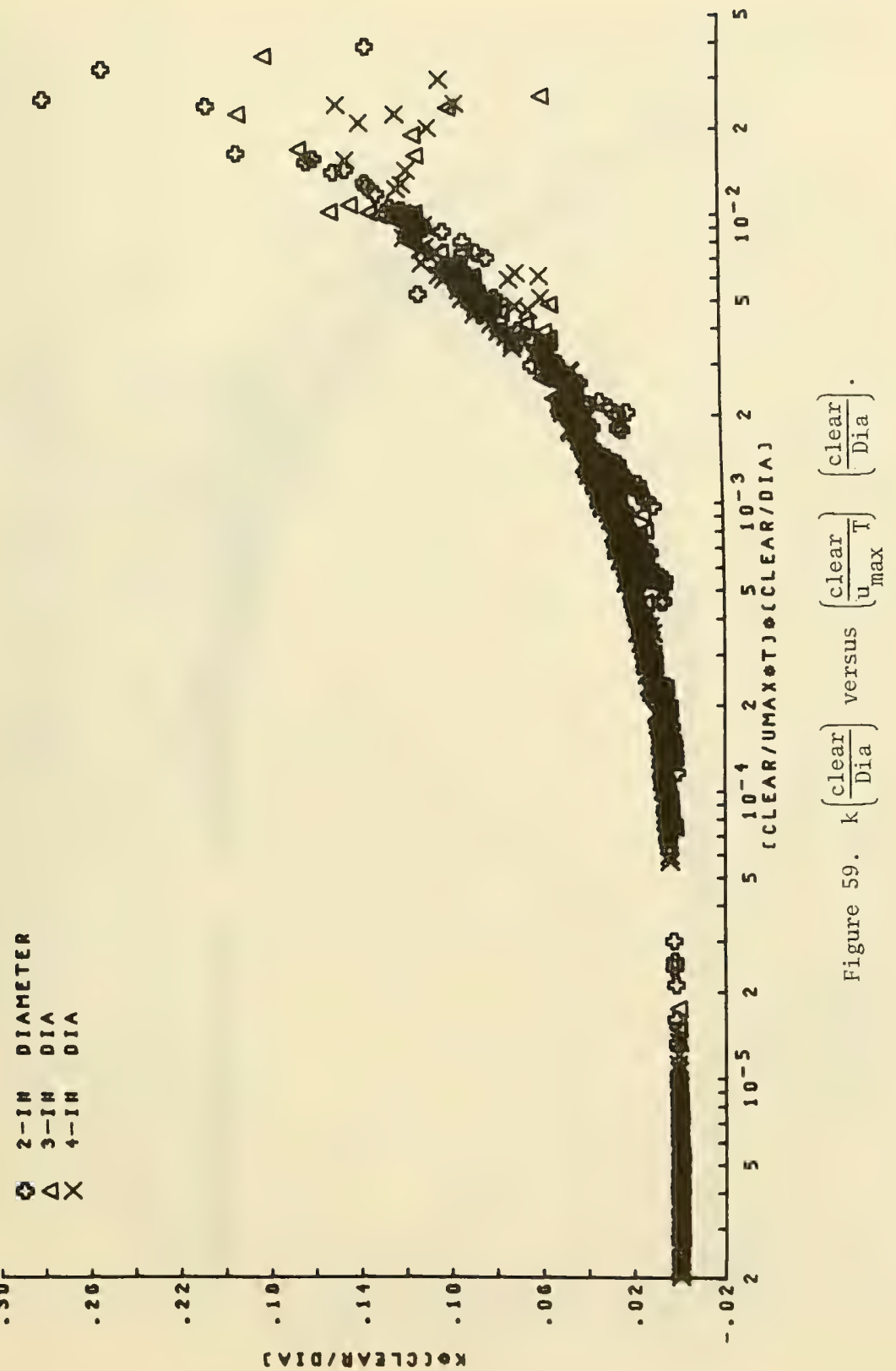


5

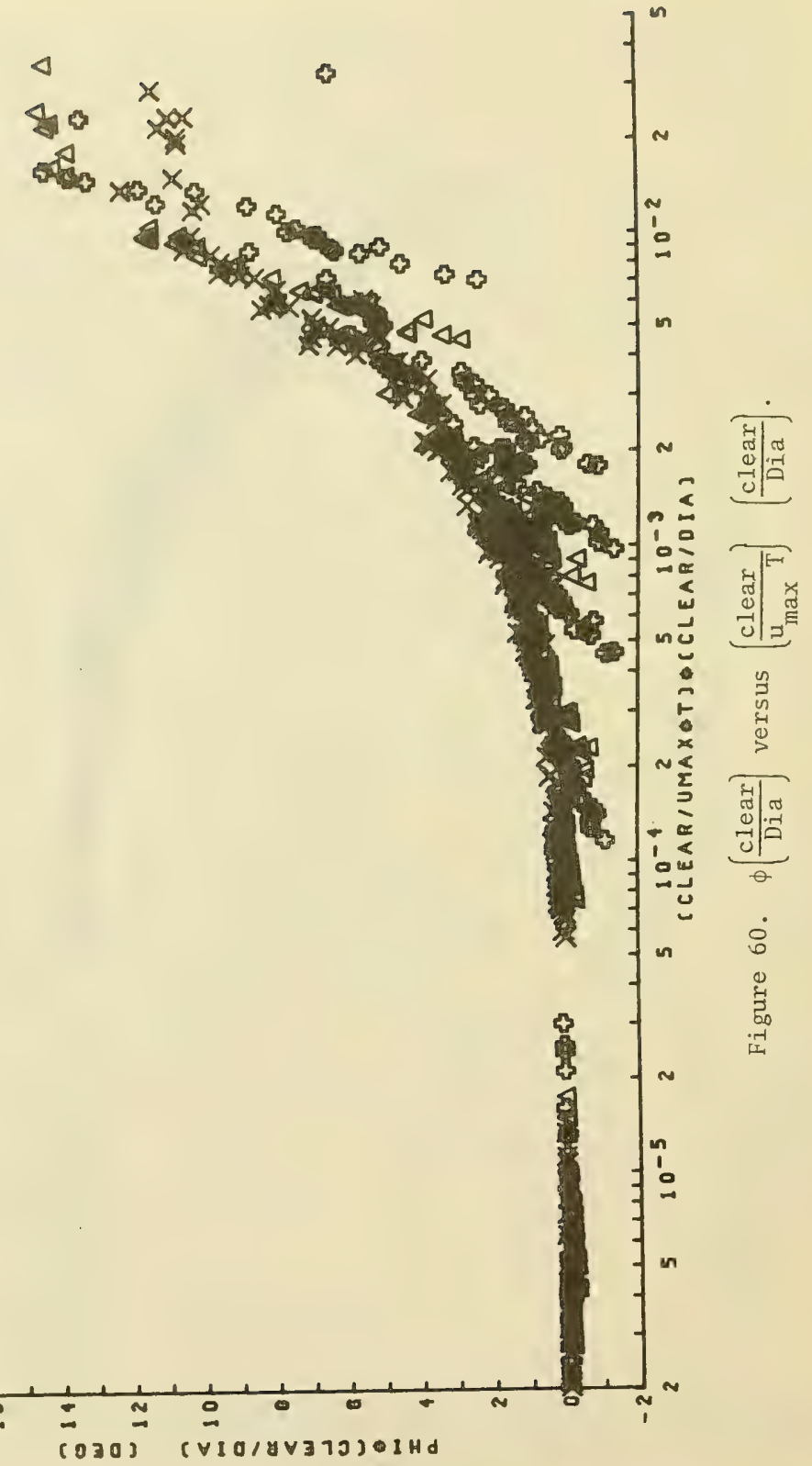




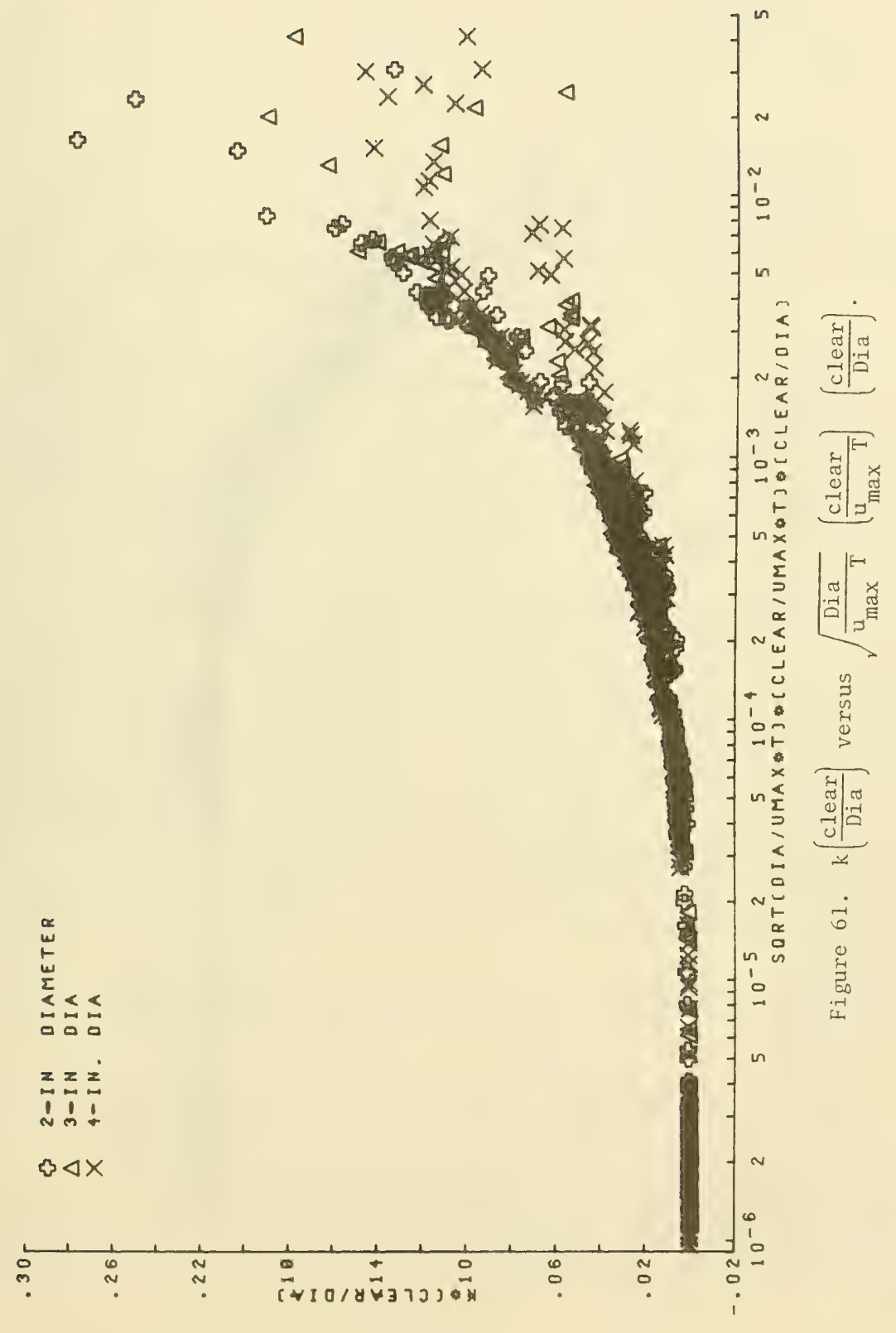




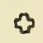

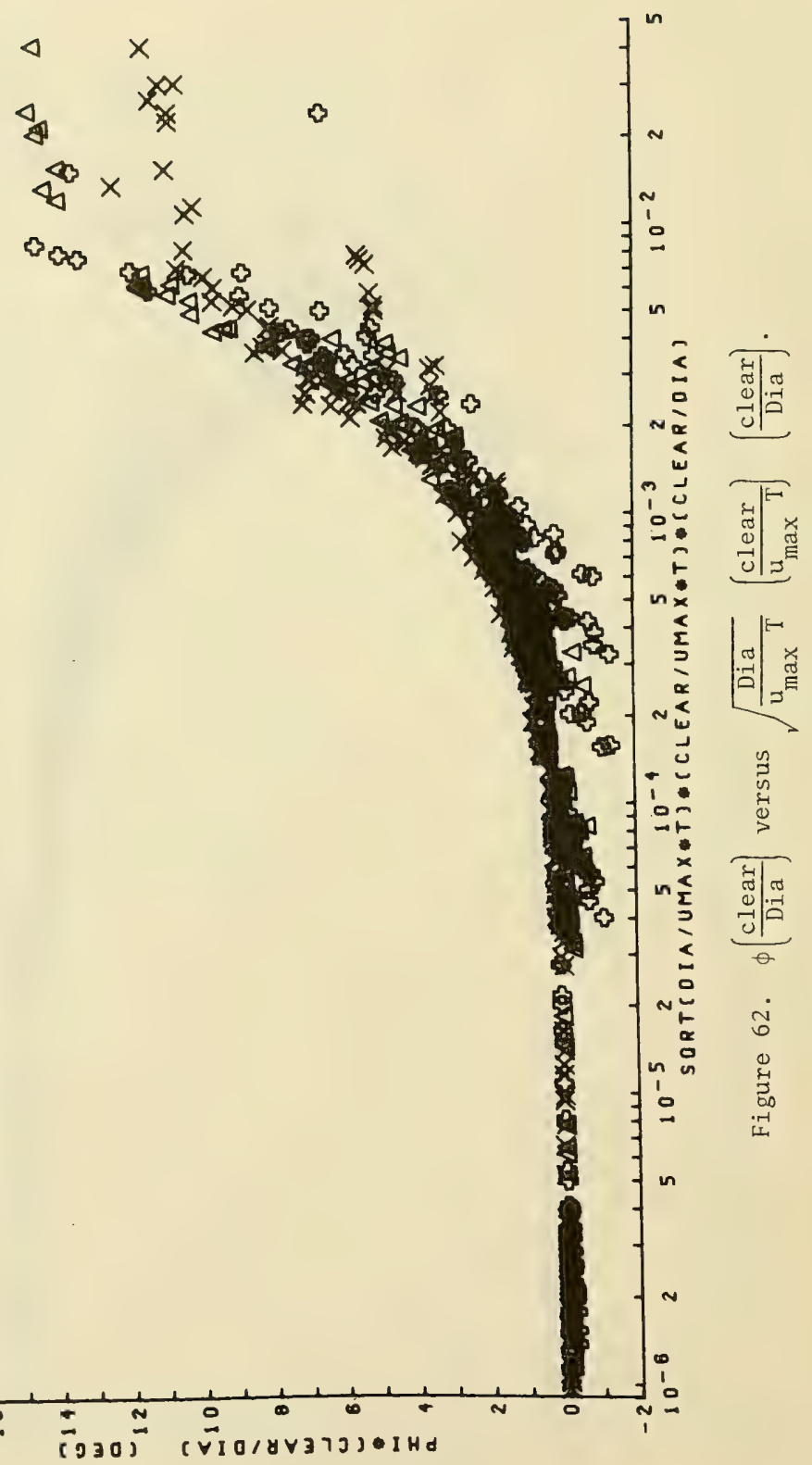


Both $\phi$ (clear/Dia) and $\mathrm{k}$ (clear/Dia) are correlated with the dimensionless combinations (clear/u max T) (clear/Dia) and $\sqrt{\mathrm{Dia} / \mathrm{u}_{\max } \mathrm{T}}$ (clear/u $\left.\mathrm{u}_{\text {max }} \mathrm{T}\right)(\mathrm{clear} / \mathrm{Dia})$. However, $\mathrm{k}$ (clear/Dia) appears to be better correlated with the first parameter, while $\phi$ (clear/Dia) shows better correlation with the second parameter.

It is clear that for values of the dimensionless parameters lower than those shown on the plots, both $\phi$ (clear/Dia) and $k$ (clear/Dia) will remain at a value of zero. This would correspond to situations where the clearance was minimal relative to the horizontal velocities, wave periods, and horizontal excursions of the water particles. Thus, both $\mathrm{k}$ and $\phi$ would be expected to equal zero and $0^{\circ}$, respectively, and the relative clearance would either equal or approach zero.

Large values of the dimensionless parameters correspond to situations where the clearance is large relative to the horizontal velocities, wave periods, and horizontal excursions of the water particles. For these cases, $\mathrm{k}$ and $\phi$ will remain at maximum values of 1 and $90^{\circ}$, respectively, while the relative clearance, (clear/Dia), will increase with increasing values of the dimensionless parameters. But as the relative clearance is increased beyond this point, the lift forces will decrease to zero, so extension of the plotted relationships to much larger values of the dimensionless parameters is of little value.

\section{Relationships Between the Coefficients of Lift and Parameters Defining the Wave and Pipeline Conditions.}

The coefficient of 1 ift, $C_{L}$, the effective positive coefficient of 1ift, $C_{L}(1-k)$, the effective negative coefficient of lift, $C_{L}(k)$, and the maximum effective coefficient of lift (maximum of $\mathrm{C}_{\mathrm{L}}(1-\mathrm{k})$ or $\mathrm{C}_{\mathrm{L}}(\mathrm{k})$ ) were plotted against various combinations of the dimensionless parameters. The parameter, $\left(\mathrm{clear} / \mathrm{u}_{\max } \mathrm{T}\right)\left(\mathrm{Dia} / \mathrm{u}_{\max } \mathrm{T}\right)$, which previously gave the best correlations with $\phi$ and $\mathrm{k}$ also demonstrated the best correlation with $\mathrm{C}_{\mathrm{L}}, \mathrm{C}_{\mathrm{L}}(1-\mathrm{k})$, and $\mathrm{C}_{\mathrm{L}}(\mathrm{k})$. However, these relationships exhibited more scatter than the previously discussed interrelationships between the coefficients of $1 \mathrm{ift}$ and the parameters, $k$ and $\phi$, so it is suggested that the previously discussed relationships be used for design purposes.

7. Relationships Between the Lift Forces and Parameters Defining the Wave and Pipeline Conditions.

As with the coefficient of lift, the total lift force $\left(\mathrm{F}_{\mathrm{L}}=\right.$ $1 / 2 \mathrm{C}_{\mathrm{L}} \rho \mathrm{A} \mathrm{u}_{\max }{ }^{2}$ ) can be partitioned into the maximum positive lift, $\mathrm{F}_{\mathrm{L}}(1-\mathrm{k})$, and the maximum negative lift, $\mathrm{F}_{\mathrm{L}}(\mathrm{k})$ (Fig. 6). These three forces, as well as the maximum lift force (maximum of either $F_{L}(1-k)$ or $\mathrm{F}_{\mathrm{L}}(\mathrm{k})$ ) were plotted against various combinations of the dimensionless parameters. Only one relationship exhibited good correlations for tine data from all three diameters plotted together. This was the Reynolds number, $u_{\max } \mathrm{Dia} / v$, versus the maximum lift force (either $F_{L}(1-k)$ or $F_{L}(k)$, whichever is greater) (Fig. 63). 


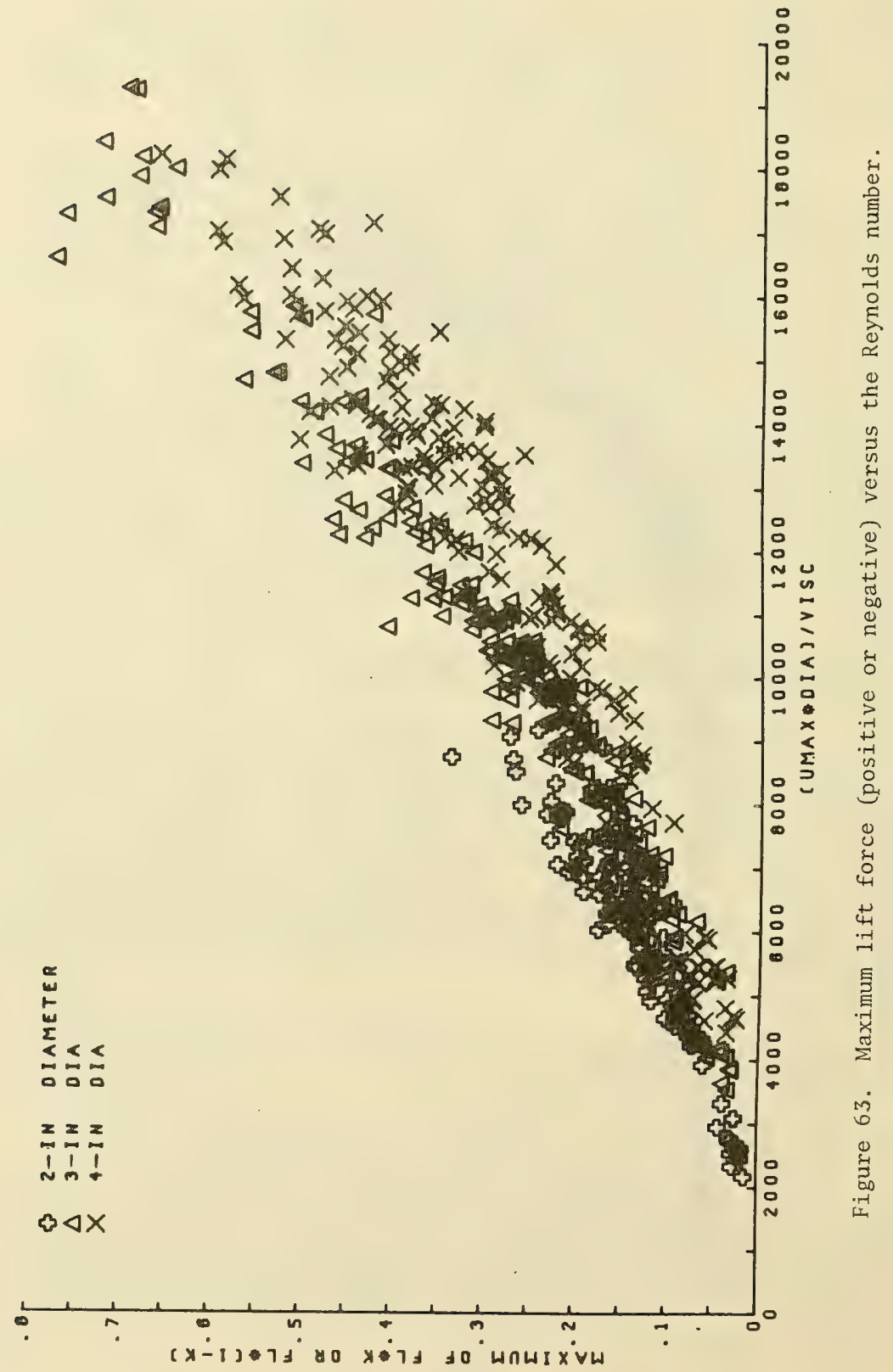


This relationship shows that for any pipe diameter, orientation angle, or bottom clearance, the maximum 1 ift force increases with the Reynolds number in a regular manner, at least over the range of the data in this investigation. The maximum lift force may occur in either the upward or downward direction, depending on the magnitude of the bottom clearance relative to the wave conditions and pipe size. This relationship does not hold for the maximum upward lift or maximum downward lift alone, but only for the largest of these two forces in any given situation.

\section{Relationships Involving the Vertical Coefficients of Mass and Drag and the Vertical Inertial and Drag Forces.}

Both the vertical coefficient of mass and the vertical inertial forces were plotted against several dimensionless parameters defining the wave and pipeline conditions, but no useful relationships were found. This is not surprising when considering that the vertical inertial forces are relatively small, and thus subject to error from the transverse eddy-induced forces which were not accounted for in the least squares analysis.

No attempt was made to plot relationships involving the vertical drag forces or drag coefficients, since these forces were negligible.

9. Relationships Between the Horizontal Coefficient of Mass and Parameters Describing the Wave and Pipeline Conditions.

A limited number of horizontal force data were taken using the 4-inchdiameter two-dimensional model. Values of $C_{M}$ and $C_{D}$ were calculated from the least squares analysis, and an attempt was made to relate these coefficients to various dimensionless parameters describing the wave and pipeline conditions.

Figure 64 shows the horizontal coefficient of mass plotted versus the relative clearance, clear/Dia, together with the potential flow solution for a circular cylinder in the vicinity of a plane wall subject to a uniform flow with constant acceleration (Grace, 1974). The data follow the potential flow solution reasonably well, although for a given relative clearance, there appears to be some variation in the value of $\mathrm{C}_{\mathrm{M}}$ with varying wave conditions. Also, the wave force data give slightly higher values of the coefficient of mass for the highest bottom clearances tested. Although the experimental data are limited, they indicate that the potential flow solution may be very useful in determining a value for the horizontal coefficient of mass, at least for wave conditions where the inertial forces predominate over the drag forces.

However, since there was some variation in the values of $C_{M}$ for different wave conditions for the same relative clearance, an attempt was made to determine relationships between the horizontal coefficient of mass and the various dimensionless parameters defining the wave and pipeline conditions. Reasonably good correlations were found between 


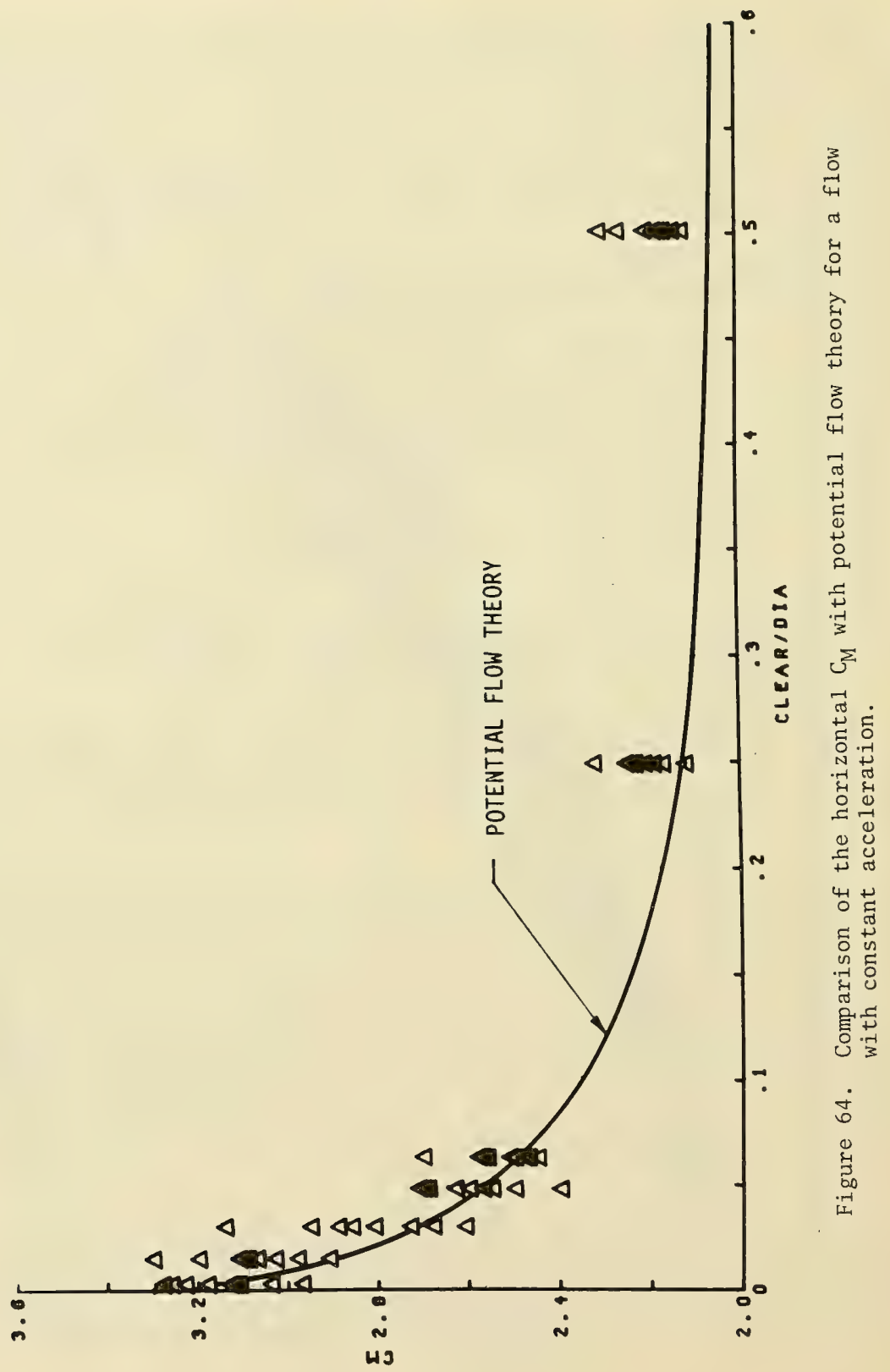


several of the parameters. Figure 65 shows the relationship for $\mathrm{C}_{M}$ versus clear $/ \mathrm{u}_{\max } \mathrm{T}$.

10. Relationships Involving the Horizontal Coefficient of Drag.

The horizontal coefficient of drag was plotted against several dimensionless parameters, but no useful relationships were found. This was expected since the horizontal drag forces in this investigation were much smaller than the inertial forces, due to the limited horizontal excursions of the water particles relative to the diameter of the pipeline.

11. Example Problems.

GIVEN: A design wave with height, $H=10$ feet and period, $T=10$ seconds acts on a pipeline with a diameter, Dia $=8$ feet in a water depth, $d=80$ feet. The pipeline is oriented at an angle of $30^{\circ}$ with respect to the wave crests. Section A of the pipeline is in contact with the bottom; section B spans the bottom at a clearance, clear $=6$ inches.

FIND: For both sections $A$ and $B$, find

(a) the values of the lift force parameters $\left(\mathrm{C}_{\mathrm{L}}, \phi\right.$, and $\left.\mathrm{k}\right)$;

(b) the maximum positive and negative lift forces;

(c) the positions of these maximum 1 ift forces in the wave cycle; and

(i) the lift force at $\theta=120^{\circ}$ in the wave cycle.

\section{SOLUTION :}

$\mathrm{L}_{\mathrm{O}}=\frac{\mathrm{g} \mathrm{T}^{2}}{2}=5.12(10)^{2}=512 \mathrm{feet}$

$\frac{\mathrm{d}}{\mathrm{L}_{\mathrm{O}}}=\frac{80}{512}=0.1562$

Using tables, $\frac{\mathrm{d}}{\mathrm{L}}=0.1885$, so $\mathrm{L}=\frac{80}{0.1885}=424 \mathrm{feet}$

$\sinh \frac{2 \pi d}{L}=1.481$

$z$ = distance from bottom to center of pipe sections. 


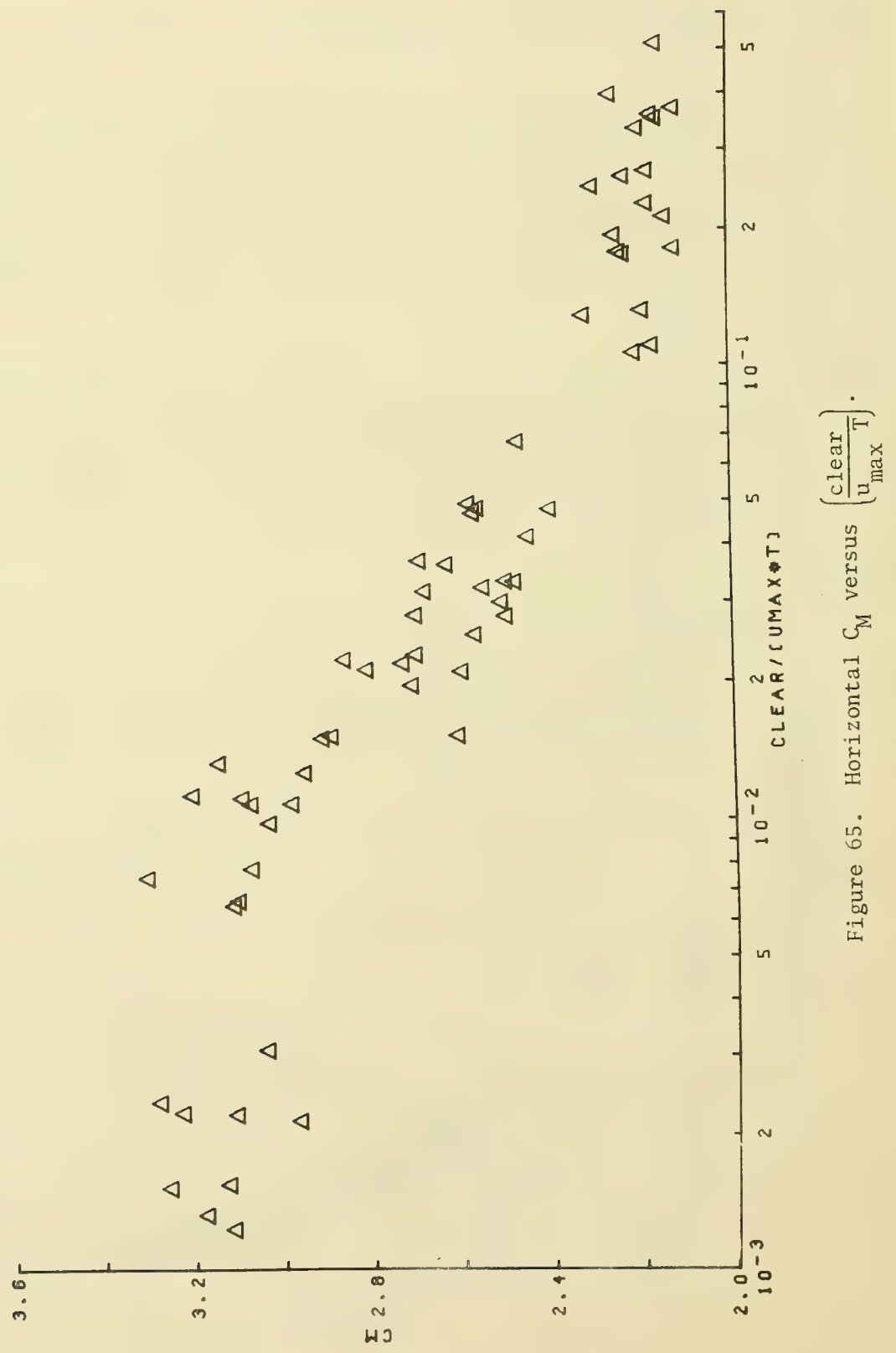


For section A (clear $=0)$

$z=4$ feet

$\frac{z}{L}=\frac{4}{424}=0.00943$

From tables, $\cosh \frac{2 \pi z}{\mathrm{~L}}=1.0017$

$u_{\max }=\frac{\pi H}{T} \frac{\cosh \left(\frac{2 \pi z}{L}\right)}{\sinh \left(\frac{2 \pi d}{L}\right)}=\frac{\pi(10)(1.0017)}{(10)(1.481)}=2.12$ feet per second

Component of $u_{\max }$ perpendicular to the pipeline axis is

$$
u_{\max }\left(\cos 30^{\circ}\right)=(2.12)(0.866)=1.84 \text { feet per second }
$$

(a) Since the pipe is in contact with the bottom, (clear $=0$ ), $\phi=0^{\circ}$ and $\mathrm{k}=0$. From Figure $40, \mathrm{C}_{\mathrm{L}}=4.5$.

(b) Maximum positive lift (per unit length)

$$
\begin{aligned}
\mathrm{F}_{\mathrm{L}}(1-\mathrm{k}) & =\frac{1}{2} \mathrm{C}_{\mathrm{L}} \rho \mathrm{A} \mathrm{u}_{\max }{ }^{2}(1-\mathrm{k}) \\
& =\frac{1}{2}(4.5)(2)(8)(1.84)^{2}(1-0) \\
& =121.9 \text { pounds per foot. }
\end{aligned}
$$

Maximum negative lift (per unit length)

Since $k=0$, there is no negative 1 ift, and the lift force is positive throughout the wave cycle.

(c) Since $\phi=0^{\circ}$, the positive lift forces are maximum at $0^{\circ}$ and $180^{\circ}$ in the wave cycle (under the crests and troughs), corresponding to the points of maximum horizontal velocities.

The lift does not become negative, but diminishes to zero at $90^{\circ}$ and $270^{\circ}$, the positions of horizontal flow reversal in the wave cycle.

(d) At $\theta=120^{\circ}$

$$
\begin{aligned}
\mathrm{F}_{\mathrm{L}} & =\frac{1}{2} \mathrm{C}_{\mathrm{L}} \rho \mathrm{A} \mathrm{u}_{\max }{ }^{2}\left[\cos ^{2}(\theta-\phi)-\mathrm{k}\right] \\
& =\frac{1}{2}(4.5)(2)(8)(1.84)^{2}\left[\cos ^{2}\left(120^{\circ}-0^{\circ}\right)-0\right] \\
& =30.5 \text { pounds per foot }
\end{aligned}
$$


For section B (clear $=6$ inches)

$$
\begin{aligned}
& z=4.5 \text { feet } \\
& \frac{z}{L}=\frac{4.5}{424}=0.0106
\end{aligned}
$$

From tables, $\cosh \frac{2 \pi \mathrm{z}}{\mathrm{L}}=1.0022$

$$
u_{\max }=\frac{\pi H}{T} \frac{\cosh \left(\frac{2 \pi z}{L}\right)}{\sinh \left(\frac{2 \pi d}{L}\right)}=\frac{\pi(10)(1.0022)}{(10)(1.481)}=2.13 \text { feet per second }
$$

component of $u_{\max }$ perpendicular to the pipeline axis is

$$
u_{\max }\left(\cos 30^{\circ}\right)=(2.13)(0.866)=1.84 \text { feet per second }
$$

(a) Use Figure 57 to determine a value for $k$

$\left(\frac{c l e a r}{u_{\max }}\right)\left(\frac{\text { Dia }}{u_{\max } T}\right)=\frac{(0.5)(8)}{(1.84)(10)(1.84)(10)}=0.0118$

so from Figure 57, $k=0.57$

and from Figure 58, $\phi=45^{\circ}$.

Alternatively, either $\phi$ or $k$ could be determined from Fig. 39, once the other is known.

From Figure 46, for $k=0.67$,

$$
\begin{gathered}
C_{L}(1-k)=2.75 \\
\text { so } C_{L}=\frac{2.75}{(1-0.67)}=8.3 .
\end{gathered}
$$

(b) Maximum positive lift (per unit length)

$$
\begin{aligned}
\mathrm{F}_{\mathrm{L}}(1-\mathrm{k}) & =\frac{1}{2} \mathrm{C}_{\mathrm{L}} \rho \mathrm{A}_{\max }{ }^{2}(1-\mathrm{k}) \\
& =\frac{1}{2}(8.3)(2)(8)(1.84)^{2}(1-0.67) \\
& =74.2 \text { pounds per foot }
\end{aligned}
$$


Maximum negative lift (per unit length)

$$
\begin{aligned}
-\mathrm{F}_{\mathrm{L}}(\mathrm{k}) & =-\frac{1}{2} \mathrm{C}_{\mathrm{L}} \rho \mathrm{A} \mathrm{u} \max ^{2}(\mathrm{k}) \\
& =-\frac{1}{2}(8.3)(2)(8)(1.84)^{2}(0.67) \\
& =-150.6 \text { pounds per foot }
\end{aligned}
$$

(c) Since $\phi=45^{\circ}$, the positive lift forces are maximum at $0^{\circ}+45^{\circ}=$ $45^{\circ}$ and $180^{\circ}+45^{\circ}=225^{\circ}$ in the wave cycle, and the negative lift forces are maximum at $90^{\circ}+45^{\circ}=135^{\circ}$ and $270^{\circ}+45^{\circ}=315^{\circ}$ in the wave cycle.

(d) At $\theta=120^{\circ}$

$$
\begin{aligned}
\mathrm{F}_{\mathrm{L}} & =\frac{1}{2} \mathrm{C}_{\mathrm{L}} \rho \mathrm{A} \mathrm{u}_{\max }{ }^{2}\left[\cos ^{2}\left(120^{\circ}-45^{\circ}\right)-0.67\right] \\
& =\frac{1}{2}(8.3)(2)(8)(1.84)^{2}\left[\cos ^{2}\left(120^{\circ}-45^{\circ}\right)-0.67\right] \\
& =-135.6 \text { pounds per foot }
\end{aligned}
$$

Again, it should be stressed that the relationships involving the lift force parameters, $C_{L}, \phi$, and $k$, were determined from model studies conducted at much lower values of the Keulegan-Carpenter parameter and Reynolds number than those encountered in full-scale situations in the ocean. Therefore, caution should be used in extrapolating these results to prototype designs.

Further studies using a larger scale facility are necessary to evaluate the importance of scale effects in these relationships, to determine their limitations, and possibly to extend or modify them so they are valid for any scale.

\section{CONCLUSIONS}

1. The traditional steady-flow lift force model, expressed as $F_{L}=1 / 2 C_{L} \rho A u^{2}$, is not a suitable model for the description of waveinduced lift forces. This model assumes that the lift force acts in one direction only (upward or downward) throughout the entire wave cycle.

2. For pipelines located at a small clearance above the bottom, a viscous choking effect limits the maximum velocities through the constriction formed by the bottom clearance. Correspondingly, the pressure drop on the bottom side of the pipe section is also limited.

In contrast, the flow velocities and corresponding pressure drop over the top side of the pipeline are not limited. As the choking effect develops and the flow becomes restricted through the bottom 
clearance constriction, more of the flow must be diverted over the top of the pipe section, resulting in a downward shift in the stagnation point, as well as an increase in the flow velocities and associated pressure drop over the top side of the pipeline.

The induced changes in the flow pattern, velocities, and associated pressure distribution over the pipe section due to choking through the bottom clearance constriction result in an upward lift force, rather than the downward lift force predicted by potential flow theory.

3. Thus, for an oscillatory wave-induced flow, the lift force acts downward in those parts of the wave cycle where the horizontal water particle velocities are not high enough to produce choking through the bottom clearance. In this case, the unrestricted flow is faster through the bottom clearance constriction than over the top of the pipe section, so the corresponding pressure distribution results in a negative lift toward the bottom boundary.

However, in those parts of the wave cycle where the horizontal velocities are sufficient to induce choking through the bottom clearance constriction, the lift force acts in an upward direction.

4. For a given pipe diameter and wave condition, as the bottom clearance is increased, higher velocities are necessary to produce the choking effect. Thus, the negative lift force can reach a greater magnitude and occur later into the wave cycle before the choking condition is induced.

Correspondingly, the positive lift that occurs only after the choking condition develops is limited to a smaller part of the wave cycle, and the maximum magnitude of these forces decreases with increasing clearance. In addition, since there is a small timelag involved in the development of the choking phenomenon and the transition from negative to positive lift, the maximum positive lift occurs later into the wave cycle, although its magnitude is diminishing.

5. A11 major features of the wave-induced lift force phenomenon can be described adequately by a modified 1 ift force equation, $\mathrm{F}_{\mathrm{L}}=$ $1 / 2 \mathrm{C}_{\mathrm{L}} \rho \mathrm{A} \mathrm{u}_{\max }{ }^{2}\left[\cos ^{2}(\theta-\phi)-\mathrm{k}\right]$, where $\phi$ represents a phase shift in the position of the maximum positive (upward) lift force relative to the point of maximum horizontal velocity at the center of the wave crest, and $k$ represents the proportion of the total lift force cycle that acts in the negative (downward) direction. The values of $\phi$ and $k$ vary from $0^{\circ}$ and 0 , respectively, for the case of a pipeline touching the bottom, and increase with increasing clearance (for a given pipeline and wave condition) to maximum values of $90^{\circ}$ and 1 , respectively, when the pipeline is far enough from the bottom so that the choking condition does not develop. $\phi=0^{\circ}$ and $k=0$ correspond to 1 ift forces that are positive throughout the wave cycle, with maximums occurring at the points of maximum horizontal velocity under the wave crests and troughs. $\phi=90^{\circ}$ and $\mathrm{k}=1$ correspond to negative lift forces throughout the wave 
cycle, with maximum downward forces occurring under the crests and troughs of the passing waves. These two cases represent the extreme conditions bounding the lift force phenomena. At any intermediate clearance between these limiting cases, both positive and negative lift forces will occur at different parts of the wave cycle, and the positions of the maximum upward and downward lift forces will not coincide with the positions of maximum horizontal velocities in the wave cycle.

In order to use this lift force model, values of the parameters, $\mathrm{C}_{\mathrm{L}}, \phi$, and $\mathrm{k}$, must be determined for the given set of wave and pipeline conditions. A model investigation was carried out to determine relationships between these parameters and various dimensionless parameters defining the wave and pipeline conditions.

\section{A direct relationship was found between the lift force} parameters, $\phi$ and $k$. Relationships were also found between the coefficient of lift, $C_{L}$, and both $\phi$ and $k$. In addition, $C_{L}$ can be partitioned into the positive effective coefficient of 1 ift, $C_{L}(1-k)$, and the effective negative coefficient of 1 ift, $C_{L}(k)$. Both of these parameters are also related to both $\phi$ and $k$. The correlation is better with $k$ than $\phi$ for the relationships involving $C_{L}, C_{L}(1-k)$, and $C_{L}(k)$.

A11 of these relationships were the same for all pipe diameters, bottom clearances, and wave conditions tested.

7. The average value of $\mathrm{C}_{\mathrm{L}}$ at $\mathrm{k}=0$ and $\phi=0^{\circ}$ (which corresponds to a pipeline in contact with the bottom with no clearance) is 4.5 . This is the same as the potential flow solution for the lift force on a circular cylinder against a plane wall subject to a steady, inviscid flow parallel to the wall.

8. Maximum values of $\mathrm{C}_{\mathrm{L}}$ occur at $\mathrm{k}=1 / 2$ and $\phi=30^{\circ}$, where $\mathrm{C}_{\mathrm{L}}=9$. In the interval from $\mathrm{k}=0$ to $1 / 2$ and $\phi=0^{\circ}$ to $30^{\circ}$, the effective positive coefficient of 1 ift $C_{L}(1-k)$ remains at approximately 4.5, while the effective negative coefficient of lift $C_{L}(k)$ increases from 0 to 4.5 . In the interval from $k=1 / 2$ to 1 and $\phi=30^{\circ}$ to $90^{\circ}$, $\mathrm{C}_{\mathrm{L}}(1-\mathrm{k})$ decreases to 0 , while $\mathrm{C}_{\mathrm{L}}(\mathrm{k})$ increases to reach a maximum of about 6 or 7 at $k=0.75$ and $\phi=45^{\circ}$, and then decreases to a maximum of 4.5 at $k=1$ and $\phi=90^{\circ}$.

9. Using the above relationships between $\mathrm{C}_{\mathrm{L}}, \phi$, and $\mathrm{k}$, if either $\phi$ or $k$ is known, the remaining two parameters can be determined.

Therefore, an attempt was made to find relationships between $\phi$ and $k$ and various dimensionless parameters defining the wave and pipeline conditions.

The best correlation was found in the relationships between $\phi$ and $k$ and the parameter clear/ $u_{\text {max }} \mathrm{T}$ for constant values of the relative clearance, clear/Dia. However, comparison of the data corresponding to the different pipe diameters indicates a slight scale effect is present. 
$\phi$ and $k$ were also related to the parameter $u_{\max } c l e a r / \nu$ for constant values of clear/Dia, although the scale effect was worse for these relationships. $\phi$ and $k$ showed very good correlation with the KeuleganCarpenter parameter, $u_{\max } \mathrm{T} / \mathrm{Dia}$, although these relationships were the same for a constant absolute clearance, rather than a constant relative clearance. Correlation between $\phi$ and $\mathrm{k}$ and the Reynolds number was poor.

10. Because a scale effect was evident in the above relationships, several of the dimensionless parameters were combined to form new dimensionless parameters that contained a11 of the important variables (clear, Dia, $u_{\max }$, and $\mathrm{T}$ ). An attempt was made to find a single parameter that was related to either $\phi$ or $k$ for all wave and pipeline conditions tested in this investigation.

Both $\phi$ and $\mathrm{k}$ showed very good correlation with the parameter $\left(\mathrm{clear} / \mathrm{u}_{\max } \mathrm{T}\right)\left(\mathrm{Dia} / \mathrm{u}_{\max } \mathrm{T}\right)$. These relationships were valid for all pipe diameters, bottom clearances, orientation angles, and wave conditions tested.

In addition, the relative clearance was combined with both $\phi$ and $k$ to form the quantities $\phi(c l e a r / D i a)$ and $k(c l e a r / D i a)$, both of which exhibited very good correlation with more of the dimensionless combinations than either $\phi$ or $\mathrm{k}$ alone. $\mathrm{k}(\mathrm{clear} / \mathrm{Dia})$ was best correlated with $\left(c l e a r / u_{\max } \mathrm{T}\right)(\mathrm{clear} / \mathrm{Dia}) . \phi(\mathrm{clear} / \mathrm{Dia})$ was also correlated with this parameter, but exhibited better correlation with the parameter $\sqrt{\mathrm{Dia} / \mathrm{u}_{\max } \mathrm{T}}\left(\mathrm{clear} / \mathrm{u}_{\max } \mathrm{T}\right)(\mathrm{clear} / \mathrm{Dia})$.

11. $\mathrm{C}_{\mathrm{L}}, \mathrm{C}_{\mathrm{L}}(1-\mathrm{k})$, and $\mathrm{C}_{\mathrm{L}}(\mathrm{k})$ were correlated with the same parameter as $\phi$ and $k$, (clear/ $\left.u_{\max } \mathrm{T}\right)\left(\mathrm{Dia} / \mathrm{u}_{\max } \mathrm{T}\right)$. However, these correlations were not as good as the previous correlations between the coefficients of lift and $\mathrm{k}$ or $\phi$.

12. For a pipeline that is not paralle1 to the wave crests, the lift forces are apparently due only to the components of the horizontal water particle velocities perpendicular to the axis of the pipeline. Using this convention, consistent values of the coefficient of lift, $\mathrm{C}_{\mathrm{L}}$, are obtained for all angles of orientation. In addition, the relationships between the lift force parameters $\mathrm{C}_{\mathrm{L}}, \phi$, and $\mathrm{k}$, as well as relationships between these parameters and various dimensionless parameters defining the wave and pipeline conditions, are identical for all angles of orientation.

13. The maximum lift force $\left(F_{L}(1-k)\right.$ or $F_{L}(k)$, whichever is greater) exhibited good correlation with the Reynolds number, ( $\left.u_{\max } \mathrm{Dia} / \nu\right)$. This relationship did not hold for the maximum positive ift $\left(\mathrm{F}_{\mathrm{L}}(1-\mathrm{k})\right.$ ) or the maximum negative lift $\left(F_{L}(k)\right)$ alone, but only for the largest of these two forces in any situation. The relationship was the same for all diameters over the range of conditions tested. 
14. The horizontal coefficient of mass, $\mathrm{C}_{\mathrm{M}}$, showed excellent agreement with the potential flow solution for a circular cylinder in the vicinity of a plane wall subject to a uniform flow with constant acceleration. These results indicate that the potential flow solution may be useful for selecting a value of $\mathrm{C}_{\mathrm{M}}$ for wave-induced forces, at least for situations in which the inertial forces predominate over the drag forces. The horizontal $\mathrm{C}_{\mathrm{M}}$ was also correlated with several of the dimensionless parameters defining the wave and pipeline conditions, such as the parameter clear/ $u_{\max } \mathrm{T}$.

\section{RECOMMENDATIONS FOR FURTHER RESEARCH}

1. Experiments similar to this investigation should be carried out in a larger wave tank facility. This would allow the testing of larger diameter pipelile models as well as experiments at higher Reynolds numbers and higher values of the Keulegan-Carpenter parameter. Such an investigation is necessary to determine the validity of extrapolating the results of the present study to design situations in the ocean, and to point out any weaknesses or limitations of the proposed lift force model due to scale effects.

2. It would be of interest to perform experiments to evaluate the magnitude, phase, and frequency spectra of the vertical transverse lift forces due to eddy shedding for a horizontal cylinder subject to oscillatory horizontal flow velocities. This could be done by oscillating a test cylinder horizontally in still water away from a boundary, or by using a pulsating flume facility. The horizontal flow patterns at the bottom could be simulated, but without the lift force phenomenon due to the boundary. Only the transverse 1 ift forces due to eddy shedding would act in the vertical direction, so the magnitude and time history of these forces could be easily measured.

A thorough analysis of the eddy forces for different pipe diameters and flow conditions would allow an evaluation of their importance relative to the Bernoulli-type lift forces, and at the same time explain some of the variations in the vertical wave force parameters calculated from an analysis which neglected the eddy forces because they could not be separated analytically because of their random nature. Adequate knowledge of the eddy forces would allow the addition of the eddy lift

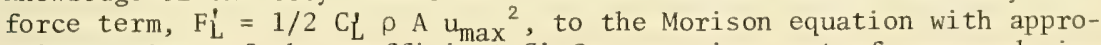
priate values of the coefficient $\mathrm{C}_{L}$ for any given set of wave and pipeline conditions.

It should be noted that evaluation of the eddy forces for a cylinder away from a boundary would only give an approximate estimate of the eddy release phenomenon for a pipe located near the bottom. The presence of the bottom boundary changes the flow pattern, velocities, and pressure distribution around the cylinder, and therefore would be expected to have some effect on the formation and release of eddies. 
3. Since the restricted flow through the narrow bottom clearance constriction is the critical part of the lift force phenomenon, the effect of pipeline roughness and bottom roughness on the wave-induced lift forces should be studied. This has practical significance, since the ocean floor is not necessarily smooth, and pipelines installed in marine waters may soon become encrusted with marine organisms, thus increasing their surface roughness.

4. The effect on the lift force phenomenon of a horizontal bottom current superimposed on the oscillatory motions of the wave action should be investigated.

5. The effect of porosity of the bottom on lift forces should also be investigated. 


\section{LITERATURE CITED}

AL-KAZILY, M.F., "Forces on Submerged Pipelines Induced by Water Waves," HEL 9-21, Hydraulic Engineering Laboratory, University of California, Berkeley, Calif., Oct. 1972.

GRACE, R.A., "Wave Forces on Submerged Objects," Report No. 10, Look Laboratory, University of Hawaii, Honolulu, Hawaii, July 1974.

MORISON, J.R., et al., "The Forces Exerted by Surface Waves on Piles," Petroleum Transactions, Vo1. 189, 1950, pp. 149-154.

PAULLING, J.R., and SIBUL, O.J., "Instrumentation for Digital Recording," Proceedings of the 15th American Towing Tank Conference, Ottawa, Canada, 1968.

YAMAMOTO, T., NAIH, J.H., and SLOTTA, L.S., "Yet Another Report on Cylinder Drag or Wave Forces on Horizontal Submerged.Cylinders," Bulletin No, 47, Engineering Experiment Station, Oregon State University, Corvallis, Oreg., Apr. 1973. 
Using Morison's method for the calculation of wave forces on a pipeline, the vertical component of the wave-induced force may be expressed as:

$$
\begin{aligned}
F_{V} & =\left(F_{I}\right)_{V}+\left(F_{D}\right)_{V}+F_{L}+F_{L}^{\prime} \\
& =C_{M} \rho V \frac{\partial V}{\partial t}+1 / 2 C_{D} \rho A v|v| \\
& +1 / 2 C_{L} \rho A u_{\max ^{2}}{ }^{2}\left[\cos ^{2}(\theta-\phi)-k\right] \\
& +1 / 2 C_{L}^{\prime} \rho A u_{\max ^{2}}{ }^{2} .
\end{aligned}
$$

Since the transverse lift force associated with eddy shedding ( $F_{L}^{\prime}$ ) is a random phenomenon, there is no way to handle its time history in analyzing a wave force record with several other forces occurring simultaneously. Because the Bernoulli-type lift forces were much larger than the eddy-associated forces for a pipeline located close to the bottom, the eddy-associated lift force term was dropped from the analysis.

The vertical components of the water particle velocities and accelerations near the bottom are small in comparison with the corresponding horizontal components. As a result, the vertical lift forces due to the horizontal components of the water particle velocities are generally much larger than the vertical drag and inertial forces. The drag forces are especially insignificant since the vertical excursions of the water particles near the bottom are smaller than the diameter of the pipeline.

Using linear wave theory, the kinematics of the wave-induced water particle motions with respect to time can be expressed as:

$$
\begin{aligned}
& u=\frac{\pi H}{T} \frac{\cosh \left(\frac{2 \pi z}{L}\right)}{\sinh \left(\frac{2 \pi d}{L}\right)} \cos \theta \\
& v=-\frac{\pi H}{T} \frac{\sinh \left(\frac{2 \pi z}{L}\right)}{\sinh \left(\frac{2 \pi d}{L}\right)} \sin \theta
\end{aligned}
$$




$$
\frac{\partial v}{\partial t}=-\frac{2 \pi^{2} H}{T^{2}} \frac{\sinh \left(\frac{2 \pi z}{L}\right)}{\sinh \left(\frac{2 \pi d}{L}\right)} \cos \theta
$$

where

$$
\begin{aligned}
& H=\text { wave height } \\
& T=\text { wave period } \\
& L=\text { wavelength } \\
& \mathrm{d}=\text { stillwater depth } \\
& \mathrm{z}=\text { vertical distance above the bottom } \\
& \theta=\frac{2 \pi t}{\mathrm{~T}}=\text { position of the wave cycle with respect to time. }
\end{aligned}
$$

Substituting these expressions into the vertical force equation yields:

$$
\begin{aligned}
F_{V}= & -C_{M}\left[\frac{\rho V 2 \pi^{2} H}{T^{2}} \frac{\sinh \left(\frac{2 \pi z}{L}\right)}{\sinh \left(\frac{2 \pi d}{L}\right)}\right] \cos \theta \\
& -C_{D}\left[\frac{\rho A \pi^{2} H^{2}}{2 T^{2}} \frac{\sinh ^{2}\left(\frac{2 \pi z}{L}\right)}{\sinh ^{2}\left(\frac{2 \pi d}{L}\right)}\right] \sin \theta \quad|\sin \theta| \\
& +C_{L}\left[\frac{\rho A \pi^{2} H^{2}}{2 T^{2}} \frac{\cosh ^{2}\left(\frac{2 \pi z}{L}\right)}{\sinh ^{2}\left(\frac{2 \pi d}{L}\right)}\right]\left[\cos ^{2}(\theta-\phi)-k\right]
\end{aligned}
$$

or

$$
F_{v}=-C_{M} F_{M v} \cos \theta-C_{D} F_{D v} \sin \theta|\sin \theta|+C_{L} F_{L v}\left[\cos ^{2}(\theta-\phi)-k\right],
$$

where

$$
\begin{aligned}
& F_{M V}=\frac{\rho V 2 \pi^{2} H}{T^{2}} \frac{\sinh \left(\frac{2 \pi z}{L}\right)}{\sinh \left(\frac{2 \pi d}{L}\right)} \\
& F_{D V}=\frac{\rho A \pi^{2} H^{2}}{2 T^{2}} \frac{\sinh ^{2}\left(\frac{2 \pi z}{L}\right)}{\sinh ^{2}\left(\frac{2 \pi d}{L}\right)}
\end{aligned}
$$




$$
F_{L V}=\frac{\rho A \pi^{2} H^{2}}{2 T^{2}} \frac{\cosh ^{2}\left(\frac{2 \pi z}{L}\right)}{\sinh ^{2}\left(\frac{2 \pi d}{L}\right)} .
$$

The expressions, $\mathrm{F}_{\mathrm{Mv}}, \mathrm{F}_{\mathrm{Dy}}$, and $\mathrm{F}_{\mathrm{Lv}}$, are constant for a given set of wave and pipeline conditions.

Linear wave theory was used in the analysis because, as discussed previously, there seems to be no obvious way of accurately describing the lift force phenomenon mathematically using higher order theories. Since the iift forces are much larger than the vertical drag or inertial forces, with the drag forces being almost completely insignificant, there was no point in using higher theories to express the vertical components of the drag and inertial forces.

For any vertical wave force record in which the corresponding wave and pipeline conditions are known, a least squares analysis can be performed on the data to determine the values of the unknown parameters $\mathrm{C}_{\mathrm{L}}, \phi, \mathrm{k}, \mathrm{C}_{\mathrm{M}}$, and $\mathrm{C}_{\mathrm{D}}$ in the vertical wave force equation. The least squares analysis yields the values of these five parameters which best fit the force data throughout the entire wave cycle. This is accomplished by determining the values of these parameters which minimizes the sum of squares of the difference between the observed force data and the corresponding forces calculated with the mathematical model throughout a complete wave cycle.

Using the appropriate trigonometric identities,

$$
\begin{aligned}
\cos ^{2}(\theta-\phi) & =1 / 2+1 / 2 \cos 2(\theta-\phi) \\
& =1 / 2+1 / 2(\cos 2 \theta \cos 2 \phi+\sin 2 \theta \sin 2 \phi),
\end{aligned}
$$

so the lift force equation can be expressed as:

$$
\begin{aligned}
& \mathrm{F}_{\mathrm{L}}=1 / 2 \mathrm{C}_{\mathrm{L}} \rho \mathrm{Au}_{\max }{ }^{2}[1 / 2 \cos 2 \phi \cos 2 \theta+1 / 2 \sin 2 \phi \sin 2 \theta+1 / 2-\mathrm{k}] \\
& \text { or } \mathrm{F}_{\mathrm{L}}=\mathrm{A}_{1} \cos 2 \theta+\mathrm{B}_{1} \sin 2 \theta+\mathrm{C}_{1}
\end{aligned}
$$

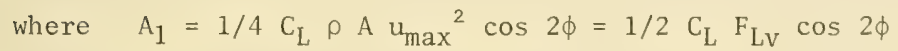

$$
\mathrm{B}_{1}=1 / 4 \mathrm{C}_{\mathrm{L}} \rho \mathrm{A} \mathrm{u}_{\max }{ }^{2} \sin 2 \phi=1 / 2 \mathrm{C}_{\mathrm{L}} \mathrm{F}_{\mathrm{LV}} \sin 2 \phi
$$




$$
C_{1}=1 / 2 C_{L} \rho A_{\max }^{2}(1 / 2-k)=C_{L} F_{L v}(1 / 2-k) .
$$

In an analogous manner, the vertical components of the inertial and drag forces can be written as:

$$
\left(F_{I}\right)_{v}=C_{M} \rho\left(\frac{\partial v}{\partial t}\right)_{\max } \cos \theta=D_{1} \cos \theta
$$

and $\left(\mathrm{F}_{\mathrm{D}}\right)_{\mathrm{V}}=1 / 2 \mathrm{C}_{\mathrm{D}} \rho \mathrm{A} v_{\max } \sin \theta\left|v_{\max } \sin \theta\right|=\mathrm{E}_{1} \sin \theta|\sin \theta|$,

where $\quad D_{1}=C_{M} \rho\left(\frac{\partial v}{\partial t}\right)_{\max }=-C_{M} F_{M v}$

$$
\begin{aligned}
& \left(\frac{\partial v}{\partial t}\right)_{\max }=-\frac{2 \pi^{2} H}{T^{2}} \frac{\sinh \left(\frac{2 \pi z}{L}\right)}{\sinh \left(\frac{2 \pi d}{L}\right)} \\
& E_{1}=1 / 2 C_{D} \rho A v_{\max }\left|v_{\max }\right|=-C_{D} F_{D v} \\
& v_{\max }=-\frac{\pi H}{T} \frac{\sinh \left(\frac{2 \pi z}{L}\right)}{\sinh \left(\frac{2 \pi d}{L}\right)} .
\end{aligned}
$$

The total vertical wave force at any position $\theta_{i}$ in the wave cycle can then be written as:

$$
\begin{aligned}
F_{v}\left(\theta_{i}\right)=F_{L}+\left(F_{I}\right)_{v}+\left(F_{D}\right)_{v}= & A_{1} \cos 2 \theta_{i}+B_{1} \sin 2 \theta_{i}+C_{1} \\
& +D_{1} \cos \theta_{i}+E_{1} \sin \theta_{i}\left|\sin \theta_{i}\right| \cdot(A 22)
\end{aligned}
$$

The parameters $A_{1}, B_{1}, C_{1}, D_{1}$, and $E_{1}$ are constant for any given values of $\mathrm{C}_{\mathrm{L}}, \phi, \mathrm{k}, \mathrm{C}_{\mathrm{M}}$, and $\mathrm{C}_{\mathrm{D}}$, corresponding to the particular wave and pipeline conditions under consideration.

The sum of squares of the differences between the observed vertical forces, $F_{o v}\left(\theta_{i}\right)$, and the corresponding calculated forces, $F_{v}\left(\theta_{i}\right)$, is 


$$
\begin{aligned}
\sum_{i=1}^{n}\left[F_{v}\left(\theta_{i}\right)-F_{o v}\left(\theta_{i}\right)\right]^{2}= & \sum_{i=1}^{n}\left[A_{1} \cos 2 \theta_{i}+B_{1} \sin 2 \theta_{i}+C_{1}+D_{1} \cos \theta_{i}\right. \\
& \left.+E_{1} \sin \theta_{i}\left|\sin \theta_{i}\right|-F_{o v}\left(\theta_{i}\right)\right]^{2} .
\end{aligned}
$$

To minimize the sum of squares of the differences, the derivative of this expression is taken separately with respect to each of the five unknown parameters $A_{1}, B_{1}, C_{1}, D_{1}$, and $E_{1}$, and the resulting expressions are set equal to zero, yielding a system of five simultaneous equations with five unknowns. The system of equations is then summed for each interval, $i$, over a complete wave cycle, and the resulting expressions are solved for the values of the unknown parameters $A_{1}, B_{1}, C_{1}, D_{1}$, and $\mathrm{E}_{1}$ which thus minimize the sum of squares of the differences. The derivatives are:

$$
\begin{aligned}
\frac{\partial\left[F_{v}\left(\theta_{i}\right)-F_{o v}\left(\theta_{i}\right)\right]^{2}}{\partial A_{1}} & =2 A_{1} \cos ^{2} 2 \theta_{i}+2 B_{1} \sin 2 \theta_{i} \cos 2 \theta_{i} \\
& +2 C_{1} \cos 2 \theta_{i}+2 D_{1} \cos \theta_{i} \cos 2 \theta_{i} \\
& +2 E_{1} \sin \theta_{i}\left|\sin \theta_{i}\right| \cos 2 \theta_{i} \\
& -2 F_{o v}\left(\theta_{i}\right) \cos 2 \theta_{i}=0 \\
\frac{\partial\left[F_{v}\left(\theta_{i}\right)-F_{o v}\left(\theta_{i}\right)\right]^{2}}{\partial B_{1}}= & 2 A_{1} \cos 2 \theta_{i} \sin 2 \theta_{i}+2 B_{1} \sin 22 \theta_{i} \\
& +2 C_{1} \sin 2 \theta_{i}+2 D_{1} \cos \theta_{i} \sin 2 \theta_{i} \\
& +2 E_{1} \sin \theta_{i}\left|\sin \theta_{i}\right| \sin 2 \theta_{i} \\
& -2 F_{o v}\left(\theta_{i}\right) \sin 2 \theta_{i}=0
\end{aligned}
$$

$$
\begin{aligned}
\frac{\partial\left[\mathrm{F}_{v}\left(\theta_{i}\right)-\mathrm{F}_{\mathrm{ov}}\left(\theta_{i}\right)\right]^{2}}{\partial \mathrm{C}_{1}} & =2 \mathrm{~A}_{1} \cos 2 \theta_{i}+2 \mathrm{~B}_{1} \sin 2 \theta_{i} \\
& +2 \mathrm{C}_{1}+2 \mathrm{D}_{1} \cos \theta_{i} \\
& +2 \mathrm{E}_{1} \sin \theta_{i}\left|\sin \theta_{i}\right| \\
& -2 \mathrm{~F}_{\mathrm{ov}}\left(\theta_{i}\right)=0
\end{aligned}
$$




$$
\begin{aligned}
\frac{\partial\left[F_{V}\left(\theta_{i}\right)-F_{O V}\left(\theta_{i}\right)\right]^{2}}{\partial D_{1}} & =2 \mathrm{~A}_{1} \cos 2 \theta_{i} \cos \theta_{i} \\
& +2 \mathrm{~B}_{1} \sin 2 \theta_{i} \cos \theta_{i}+2 \mathrm{C}_{1} \cos \theta_{i} \\
& +2 \mathrm{D}_{1} \cos ^{2} \theta_{i}+2 \mathrm{E}_{1} \sin \theta_{i}\left|\sin \theta_{i}\right| \cos \theta_{i} \\
& -2 \mathrm{~F}_{\mathrm{ov}}\left(\theta_{i}\right) \cos \theta_{i}=0
\end{aligned}
$$

$$
\begin{aligned}
\frac{\partial\left[\mathrm{F}_{v}\left(\theta_{i}\right)-\mathrm{F}_{\mathrm{ov}}\left(\theta_{i}\right)\right]^{2}}{\partial \mathrm{E}_{1}} & =2 \mathrm{~A}_{1} \cos 2 \theta_{i} \sin \theta_{i}\left|\sin \theta_{i}\right| \\
& +2 \mathrm{~B}_{1} \sin 2 \theta_{i} \sin \theta_{i}\left|\sin \theta_{i}\right| \\
& +2 \mathrm{C}_{1} \sin \theta_{i}\left|\sin \theta_{i}\right| \\
& +2 \mathrm{D}_{1} \cos \theta_{i} \sin \theta_{i}\left|\sin \theta_{i}\right| \\
& +2 \mathrm{E}_{1}\left(\sin \theta_{i}\left|\sin \theta_{i}\right|\right)^{2} \\
& -2 \mathrm{~F}_{\mathrm{ov}}\left(\theta_{i}\right) \sin \theta_{i}\left|\sin \theta_{i}\right|=0 .
\end{aligned}
$$

For an even number of equally spaced time intervals, $\theta_{i}$, summed over a complete wave cycle, many of the terms cancel out due to the symmetry of these sinusoidal functions. Thus,

$$
\begin{aligned}
& \sum_{i=1}^{n} \cos \theta_{i}=0 \\
& \sum_{i=1}^{n} \cos 2 \theta_{i}=0 \\
& \sum_{i=1}^{n} \sin 2 \theta_{i}=0 \\
& \sum_{i=1}^{n} \sin \theta_{i}\left|\sin \theta_{i}\right|=0 \\
& \sum_{i=1}^{n} \cos \theta_{i} \cos 2 \theta_{i}=0
\end{aligned}
$$




$$
\begin{aligned}
& \sum_{i=1}^{n} \cos \theta_{i} \sin 2 \theta_{i}=0 \\
& \sum_{i=1}^{n} \cos \theta_{i} \sin \theta_{i}\left|\sin \theta_{i}\right|=0 \\
& \sum_{i=1}^{n} \cos 2 \theta_{i} \sin 2 \theta_{i}=0 \\
& \sum_{i=1}^{n} \cos 2 \theta_{i} \sin \theta_{i}\left|\sin \theta_{i}\right|=0 \\
& \sum_{i=1}^{n} \sin 2 \theta_{i} \sin \theta_{i}\left|\sin \theta_{i}\right|=0
\end{aligned}
$$

when taken over a complete wave cycle, As a result, only the squared terms, and the terms involving the observed forces, $F_{O}\left(\theta_{i}\right)$, remain in these equations. The resulting expressions are:

$$
\begin{aligned}
& A_{1} \sum_{i=1}^{n} \cos ^{2} 2 \theta_{i}-\sum_{i=1}^{n} F_{o v}\left(\theta_{i}\right) \cos 2 \theta_{i}=0 \\
& B_{1} \sum_{i=1}^{n} \sin ^{2} 2 \theta_{i}-\sum_{i=1}^{n} F_{o v}\left(\theta_{i}\right) \sin 2 \theta_{i}=0 \\
& C_{1} \sum_{i=1}^{n} i-\sum_{i=1}^{n} F_{o v}\left(\theta_{i}\right)=n_{1}-\sum_{i=1}^{n} F_{o v}\left(\theta_{i}\right)=0 \\
& D_{1} \sum_{i=1}^{n} \cos ^{2} \theta_{i}-\sum_{i=1}^{n} F_{o v}\left(\theta_{i}\right) \cos \theta_{i}=0
\end{aligned}
$$




$$
\begin{aligned}
& E_{1} \sum_{i=1}^{n}\left(\sin \theta_{i}\left|\sin \theta_{i}\right|\right)^{2} \\
& -\sum_{i=1}^{n} F_{o v}\left(\theta_{i}\right) \sin \theta_{i}\left|\sin \theta_{i}\right|=0
\end{aligned}
$$

where $\mathrm{n}$ is the total number of values taken from the vertical wave force record (from an even number of equally spaced intervals per wave cycle, and over any number of complete wave cycles), and $i$ is the number of the interval.

These expressions are easily solved for the unknown parameters $A_{1}$, $\mathrm{B}_{1}, \mathrm{C}_{1}, \mathrm{D}_{1}$, and $\mathrm{E}_{1}$, yielding:

$$
\begin{aligned}
& A_{1}=\frac{\sum_{i=1}^{n} F_{o v}\left(\theta_{i}\right) \cos 2 \theta_{i}}{\sum_{i=1}^{n} \cos ^{2} 2 \theta_{i}} \\
& B_{1}=\frac{\sum_{i=1}^{n} F_{o v}\left(\theta_{i}\right) \sin 2 \theta_{i}}{\sum_{i=1}^{n} \sin ^{2} 2 \theta_{i}} \\
& C_{1}=\frac{\sum_{i=1}^{n} F_{o v}\left(\theta_{i}\right)}{\sum_{i=1}^{n} F_{o v}\left(\theta_{i}\right) \cos \theta_{i}} \\
& D_{1}=\frac{\sum_{i=1}^{n} \cos ^{2} \theta_{i}}{n}
\end{aligned}
$$




$$
E_{1}=\frac{\sum_{i=1}^{n} F_{o v}\left(\theta_{i}\right) \sin \theta_{i}\left|\sin \theta_{i}\right|}{\sum_{i=1}^{n}\left(\sin \theta_{i}\left|\sin \theta_{i}\right|\right)^{2}} .
$$

With these relationships, the corresponding values of the parameters $C_{L}, \phi, k, C_{M}$, and $C_{D}$ in the vertical wave force equation which best fit the data throughout the complete wave cycle can be obtained.

The coefficients of mass and drag, $C_{M}$ and $C_{D}$, are obtained directly from the parameters $\mathrm{D}_{1}$ and $\mathrm{E}_{1}$, since

$$
\begin{aligned}
& C_{M}=-\frac{D_{1}}{F_{M v}}=-\frac{\sum_{i=1}^{n} F_{o v}\left(\theta_{i}\right) \cos \theta_{i}}{F_{M v} \sum_{i=1}^{n} \cos ^{2} \theta_{i}} \\
& C_{D}=-\frac{E_{1}}{F_{D v}}=-\frac{\sum_{i=1}^{n} F_{o v}\left(\theta_{i}\right) \sin \theta_{i}\left|\sin \theta_{i}\right|}{F_{D v} \sum_{i=1}^{n}\left(\sin \theta_{i}\left|\sin \theta_{i}\right|\right)^{2}} .
\end{aligned}
$$

Since $A_{1}=1 / 2 C_{L} F_{L v} \cos 2 \phi$ and $B_{1}=1 / 2 C_{L} F_{L v} \sin 2 \phi$, the phase shift parameter $\phi$ can be obtained from:

$$
\phi=1 / 2(2 \phi)=1 / 2 \tan ^{-1}\left(\frac{\sin 2 \phi}{\cos 2 \phi}\right)=1 / 2 \tan ^{-1}\left(\frac{B_{1}}{A_{1}}\right)
$$

since $1 / 2 \quad C_{L} \quad F_{L v}$ cancels out of the expression $\left(\frac{B_{1}}{A_{1}}\right)$. Thus,

$$
\phi=1 / 2 \tan ^{-1}\left(\frac{\sum_{i=1}^{n} F_{o v}\left(\theta_{i}\right) \sin 2 \theta_{i} / \sum_{i=1}^{n} \sin ^{2} 2 \theta_{i}}{\sum_{i=1}^{n} F_{o v}\left(\theta_{i}\right) \cos 2 \theta_{i} / \sum_{i=1}^{n} \cos ^{2} 2 \theta_{i}}\right) .
$$


After $\phi$ is known, the coefficient of 1 ift, $C_{L}$, can be obtained from either $A_{1}$ or $B_{1}$, since

$$
\mathrm{C}_{\mathrm{L}}=\frac{\mathrm{A}_{1}}{1 / 2 \mathrm{~F}_{\mathrm{LV}} \cos 2 \phi}=\frac{\sum_{i=1}^{n} \mathrm{~F}_{\mathrm{OV}}\left(\theta_{i}\right) \cos 2 \theta_{i}}{1 / 2 \mathrm{~F}_{\mathrm{LV}} \cos 2 \phi \sum_{i=1}^{n} \cos ^{2} 2 \theta_{i}}
$$

or

$$
\mathrm{C}_{\mathrm{L}}=\frac{\mathrm{B}_{1}}{1 / 2 \mathrm{~F}_{\mathrm{Lv}} \sin 2 \phi}=\frac{\sum_{i=1}^{\mathrm{n}} \mathrm{F}_{\mathrm{ov}}\left(\theta_{i}\right) \sin 2 \theta_{i}}{1 / 2 \mathrm{~F}_{\mathrm{Lv}} \sin 2 \phi \sum_{i=1}^{n} \sin ^{2} 2 \theta_{i}} .
$$

Alternatively, $C_{L}$ could be obtained from $A_{1}$ and $B_{1}$ directly without first solving for $\phi$, since

$$
\begin{aligned}
\sqrt{A_{1}{ }^{2}+B_{1}{ }^{2}} & =\sqrt{\left(1 / 2 C_{L} F_{L V} \cos 2 \phi\right)^{2}+\left(1 / 2 C_{L} F_{L v} \sin 2 \phi\right)^{2}} \\
& =\sqrt{\left(1 / 2 C_{L} F_{L V}\right)^{2}\left(\cos ^{2} 2 \phi+\sin ^{2} 2 \phi\right)} \\
& =1 / 2 C_{L} F_{L V} .
\end{aligned}
$$

Thus,

$$
\begin{aligned}
& C_{L}=\frac{2 \sqrt{A_{1}{ }^{2}+B_{1}{ }^{2}}}{F_{L v}}
\end{aligned}
$$

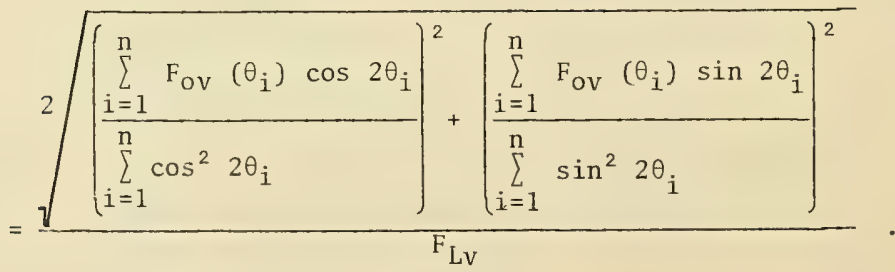


Finally, the parameter $\mathrm{k}$ can be obtained from $\mathrm{C}_{1}$ knowing the value of $\mathrm{C}_{\mathrm{L}}$, since

$$
k=1 / 2-\frac{\mathrm{C}_{1}}{\mathrm{C}_{\mathrm{L}} \mathrm{F}_{\mathrm{LV}}}=1 / 2-\frac{\sum_{\mathrm{i}=1}^{\mathrm{n}} \mathrm{F}_{\mathrm{ov}}\left(\theta_{\mathrm{i}}\right) / \mathrm{n}}{\mathrm{C}_{\mathrm{L}} \mathrm{F}_{\mathrm{Lv}}} .
$$

Thus, once the vertical wave forces on a pipeline are measured experimentally, the values of the parameters $C_{L}, \phi, k, C_{M}$, and $C_{D}$ of the vertical wave force equation which best fit the data throughout the entire wave cycle can be determined for the particular set of wave and pipeline conditions tested.

In an analogous manner, the least squares analysis car be applied to the horizontal wave force data. Omitting the horizontal force associated with eddy shedding, the horizontal component of the waveinduced force can be expressed as equation (2):

$$
F_{h}=\left(F_{I}\right)_{h}+\left(F_{D}\right)_{h}=C_{M} \rho V \frac{\partial u}{\partial t}+1 / 2 C_{D} \rho A u|u| .
$$

The data from the horizontal force measurements show that the horizontal eddy forces are insignificant in comparison to the horizontal drag and inertial forces for the experimental conditions tested.

Using linear wave theory, the horizontal components of the wave kinematics with respect to time can be expressed as:

$$
\begin{gathered}
u=\frac{\pi H}{T} \frac{\cosh \left(\frac{2 \pi z}{L}\right)}{\sinh \left(\frac{2 \pi d}{L}\right)} \cos \theta \\
\frac{\partial u}{\partial t}=-\frac{2 \pi^{2} H}{T^{2}} \frac{\cosh \left(\frac{2 \pi z}{L}\right)}{\sinh \left(\frac{2 \pi d}{L}\right)} \sin \theta .
\end{gathered}
$$


Substituting these expressions into the horizontal wave force equation yields:

$$
\begin{aligned}
F_{h} & =C_{D}\left|\frac{\rho A \pi^{2} H^{2}}{2 T^{2}}-\frac{\cosh ^{2}\left(\frac{2 \pi z}{L}\right)}{\sinh ^{2}\left(\frac{2 \pi d}{L}\right)}\right| \cos \theta|\cos \theta| \\
& -C_{M}\left|\frac{\rho V 2 \pi^{2} H}{T^{2}} \frac{\cosh \left(\frac{2 \pi z}{L}\right)}{\sinh \left(\frac{2 \pi d}{L}\right)}\right| \sin \theta
\end{aligned}
$$

or $F_{h}=C_{D} F_{D h} \cos \theta|\cos \theta|-C_{M} F_{M h} \sin \theta$

where

$$
\begin{aligned}
& F_{D h}=\frac{\rho A \pi^{2} H^{2}}{2 T^{2}} \frac{\cosh ^{2}\left(\frac{2 \pi z}{L}\right)}{\sinh ^{2}\left(\frac{2 \pi d}{L}\right)} \\
& F_{M h}=\frac{\rho V 2 \pi^{2} H}{T^{2}} \frac{\cosh \left(\frac{2 \pi z}{L}\right)}{\sinh \left(\frac{2 \pi d}{L}\right)} .
\end{aligned}
$$

The expressions $F_{D h}$ and $F_{M h}$ are constant for a given set of wave and pipeline conditions.

The horizontal component of the wave-induced force can also be written as :

$$
F_{h}=A_{2} \cos \theta|\cos \theta|+B_{2} \sin \theta
$$

where

$$
A_{2}=C_{D} F_{D h}=1 / 2 C_{D} \rho A u_{\max }\left|u_{\max }\right|
$$




$$
\begin{aligned}
& u_{\max }=\frac{\pi H}{T} \frac{\cosh \left(\frac{2 \pi z}{L}\right)}{\sinh \left(\frac{2 \pi d}{L}\right)} \\
& B_{2}=-C_{M} F_{M h}=C_{M} \rho V\left(\frac{\partial u}{\partial t}\right) \\
& \max \\
& \left(\frac{\partial u}{\partial t}\right)_{\max }=-\frac{2 \pi^{2} H}{T^{2}} \frac{\cosh \left(\frac{2 \pi z}{L}\right)}{\sinh \left(\frac{2 \pi d}{L}\right)} .
\end{aligned}
$$

Thus, the total horizontal wave force at any position $\theta_{i}$ in the wave cycle can be expressed as:

$$
\begin{aligned}
F_{h}\left(\theta_{i}\right) & =\left(F_{D}\right)_{h}+\left(F_{I}\right)_{h} \\
& =A_{2} \cos \theta_{i}\left|\cos \theta_{i}\right|+B_{2} \sin \theta_{i}
\end{aligned}
$$

where the parameters $A_{2}$ and $B_{2}$ are constant for any given values of $C_{D}$ and $\mathrm{C}_{\mathrm{M}}$, corresponding to the particular wave and pipeline conditions under consideration.

The sum of squares of the differences between the observed horizontal forces, $F_{o h}\left(\theta_{i}\right)$, and the corresponding calculated forces, $F_{h}\left(\theta_{i}\right)$, is written as:

$$
\begin{aligned}
\sum_{i=1}^{n}\left[F_{h}\left(\theta_{i}\right)-F_{o h}\left(\theta_{i}\right)\right]^{2}= & \sum_{i=1}^{n}\left[A_{2} \cos \theta_{i}\left|\cos \theta_{i}\right|\right. \\
& \left.+B_{2} \sin \theta_{i}-F_{\text {oh }}\left(\theta_{i}\right)\right]^{2} .
\end{aligned}
$$

The derivatives of this expression taken with respect to the unknown parameters $A_{2}$ and $B_{2}$ and set equal to zero give the following equations:

$$
\frac{\partial\left[F_{h}\left(\theta_{i}\right)-F_{o h}\left(\theta_{i}\right)\right]^{2}}{\partial A_{2}}=2 A_{2}\left(\cos \theta_{i}\left|\cos \theta_{i}\right|\right)^{2}
$$




$$
\begin{aligned}
& +2 \mathrm{~B}_{2} \sin \theta_{i} \cos \theta_{i}\left|\cos \theta_{i}\right| \\
& -2 \mathrm{~F}_{\text {oh }}\left(\theta_{i}\right) \cos \theta_{i}\left|\cos \theta_{i}\right| \\
& =0
\end{aligned}
$$

$$
\begin{aligned}
\frac{\partial\left[F_{h}\left(\theta_{i}\right)-F_{\text {oh }}\left(\theta_{i}\right)\right]^{2}}{\partial B_{2}}= & 2 A_{2} \cos \theta_{i}\left|\cos \theta_{i}\right| \sin \theta_{i} \\
& +2 B_{2} \sin ^{2} \theta_{i} \\
& -2 F_{o h}\left(\theta_{i}\right) \sin \theta_{i} \\
& =0 .
\end{aligned}
$$

Since $\sum_{i=1}^{n} \sin \theta_{i} \cos \theta_{i}\left|\cos \theta_{i}\right|=0$ for an even number of equally spaced intervals $\theta_{i}$ summed over a complete wave cycle, the resulting summed expressions for the derivatives set equal to zero are

$$
\begin{aligned}
& \mathrm{A}_{2} \sum_{i=1}^{n}\left(\cos \theta_{i}\left|\cos \theta_{i}\right|\right)^{2}-\sum_{i=1}^{n} F_{\text {oh }}\left(\theta_{i}\right) \cos \theta_{i}\left|\cos \theta_{i}\right|=0 \\
& \mathrm{~B}_{2} \sum_{i=1}^{n} \sin ^{2} \theta_{i}-\sum_{i=1}^{n} F_{\text {oh }}\left(\theta_{i}\right) \sin \theta_{i}=0 .
\end{aligned}
$$

These expressions are easily solved for the unknown parameters $A_{2}$ and $\mathrm{B}_{2}$, yielding:

$$
A_{2}=\frac{\sum_{i=1}^{n} F_{\text {oh }}\left(\theta_{i}\right) \cos \theta_{i}\left|\cos \theta_{i}\right|}{\sum_{i=1}^{n}\left(\cos \theta_{i}\left|\cos \theta_{i}\right|\right)^{2}}
$$




$$
B_{2}=\frac{\sum_{i=1}^{n} F_{o h}\left(\theta_{i}\right) \sin \theta_{i}}{\sum_{i=1}^{n} \sin ^{2} \theta_{i}} .
$$

The coefficients of mass and drag which best fit the horizontal wave force data throughout the entire wave cycle can thus be obtained directly from the parameters $A_{2}$ and $B_{2}$ since

$$
\begin{aligned}
& C_{D}=\frac{A_{2}}{F_{D h}}=\frac{\sum_{i=1}^{n} F_{o h}\left(\theta_{i}\right) \cos \theta_{i}\left|\cos \theta_{i}\right|}{F_{D h} \sum_{i=1}^{n}\left(\cos \theta_{i}\left|\cos \theta_{i}\right|\right)^{2}} \\
& C_{M}=-\frac{B_{2}}{F_{M h}}=-\frac{\sum_{i=1}^{n} F_{o h}\left(\theta_{i}\right) \sin \theta_{i}}{F_{M h}^{n} \sum_{i=1}^{n} \sin ^{2} \theta_{i}} .
\end{aligned}
$$


APPENDIX B

COMPUTER PROGRAM FOR VERTICAL LEAST SQUARES ANALYSIS (TWO-DIMENSIONAL DATA) 


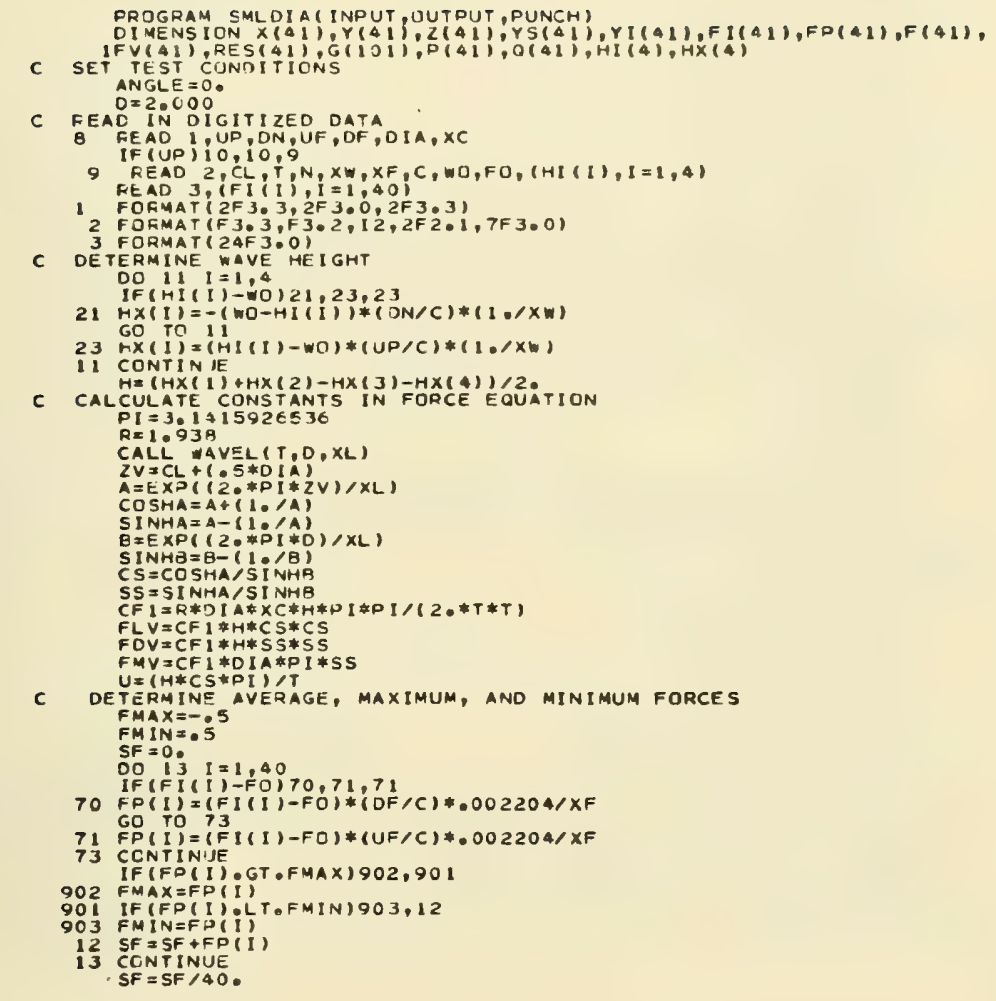


$S F F=0$.
$S F X=0$ :

SF $Y=0$.

$\mathrm{SF} Q=0$ :

SF $Z=0$.

DT $=031415926536$

$A=-D$ r

DO $15 \quad 1=1,40$

$X(I)=\cos (A)$

$B=2 * A$

$Y(I)=\cos (B)$

$C(I)=S I N(B)$

$C=S I N(A)$

$Z(1)=C$ ABS (C)

$F(I)$ sFP(I)-SF

$S F F=S F F+F(I) * F(1)$

$S F X=S F X+F(I) \neq X(1)$

$S F Y=S F Y+F(I) * Y(I)$

SF $\mathrm{O}=5 \mathrm{~F} Q+F(I) \neq O(I)$

SFZ $=S F Z+F(1) * Z(I)$

CCNTINUE

AXISFX,20.

$A Y=S F Y / 20$.

$A Q=S F Q / 20$.

$A Z \times S F Z / 15$

$V X=A X * S F X$

$Y Y=A Y * S F Y$

$V O=A O * S F O$

$V Q=A Q * S F Q$
$V Z=A Z * S F Z$

$V Z=A Z * S F Z$

c $\quad V R=S F F-V X-V Y=V Z-V O$

ALCULATE COEFFICIENTS ANO PARAMETERS PHI AND K

PHI $=28.64789 * A T A N 2$ (AO.AY)

IF (PHI.LT. 45 .) 7999,8999

7999 PHI xPHI + 180

8999 CONTINUE

$Y A=S O R T(A O * A O+A Y * A Y)$

$C L Y=2$ * *YA $Y F L V$

ANG = (ANGLE

CLVAICLVICOS (ANG)

$C L V U=C L V A / C O S(A N G)$

CMV $=-A X / F M V$

CDV $=-A Z / F D V$

$X K=.5-(S F /(C L V * F L V))$

= PRINT RESULTS OF ANALYSIS

PRINT 200

200 FCFMATIIHI)

PRINT

4 FOFMAT ( $10 X, 6 H T$ (SEC), $7 X, 6 H H T$ (FT), 5X, 9HWAVEL (FT), 6X,7HOEP(FT), $4,9 H U$ 1 MAX(FPS), $4 X$, 9HCLEAR(FT), 6X, 7HDIA (FT), 3X, 12HCYL LGTH(FT), $4 X$, BHANG(D 2EG)

PRINT $300, T, H, X L, D, U, C L, D I A, X C, A N G L E$

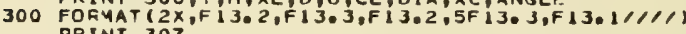

PRINT 307

307 FORMAT $16 X, 12 H T O T A L$ SUM SO, 6X, 5HCOS2A, $10 X, 5 H S I N 2 A, 11 X, 4 H C O S A, 8 X, 1 O M$ 


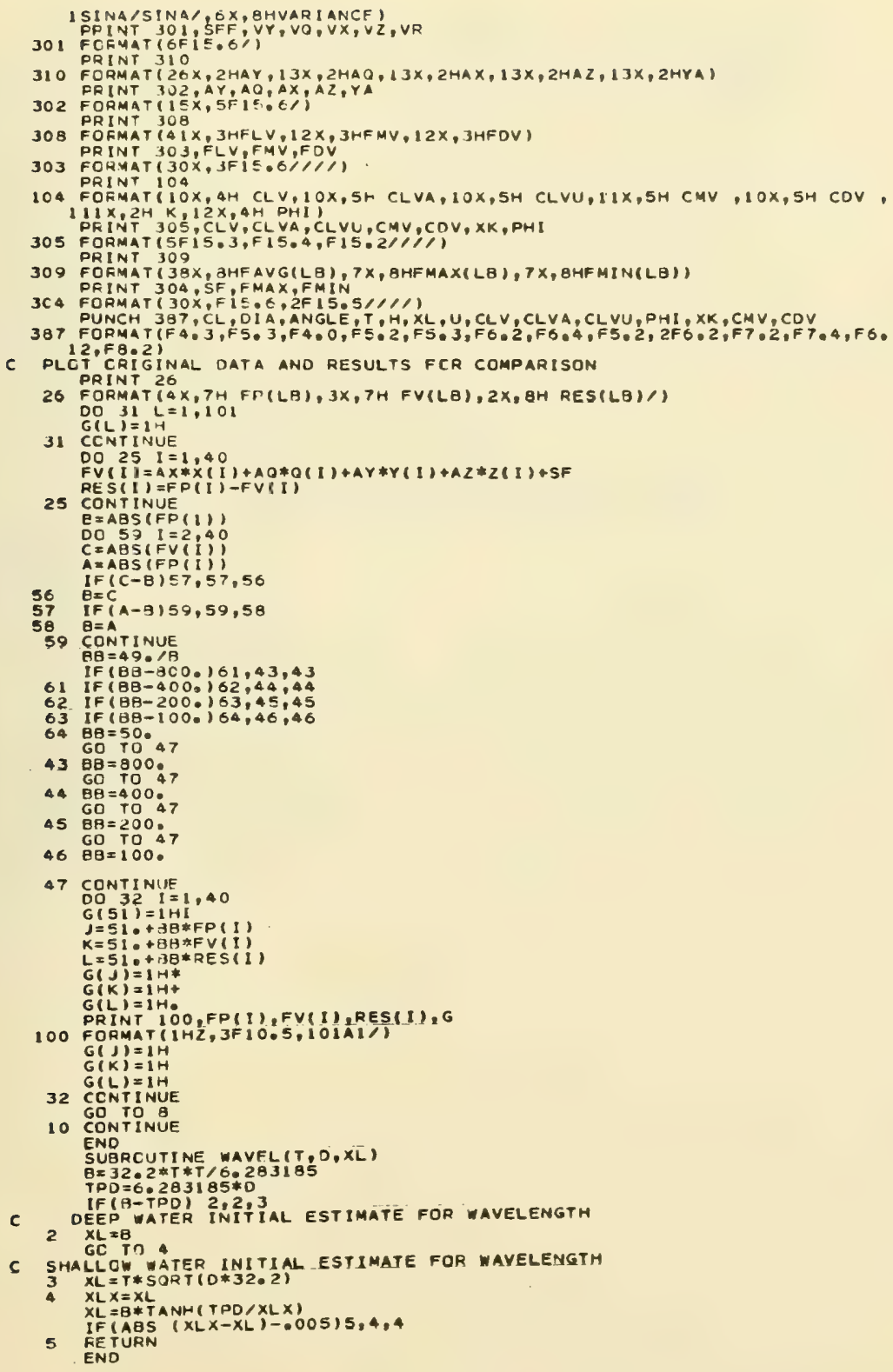


APPENDIX C

COMPUTER PROGRAM FOR VERTICAL LEAST SQUARES ANALYSIS (THREE-DIMENSIONAL DATA)

144 
PROGRAM WVFORC3 INPUT, TAPE 1 , OUTPUT, PUNCH)

DIMENSION X(150),Y(150), Z(150),0(150),FI(360),H1(380),FD(150),FV(

$S(150), G(101), H M A X(2), H M I N(2), T 1(2), T 2(2), H P(150), x T$ $2(2), I D R E C(2)$

INTEGER LABEL (B)

C SET TEST CONDITIONS

$C L=.001$

DI $A=\cdot 3333$

$X C=917$

$D=2,757$

C READ IN DIGITIZEO DATA

C WAVE CATA IS IN FT.

FORCE DATA IS IN IO-GRAMS

CALL NOBLOK (1)

1 FEAD I 1 ANGLE

IF (ANGLE $L$ L $00,110,11$

11 RE $A D$ ( 1$)$ LABEL

NREC $S=L A B E L(B)$

PRINT 200

200 FOFMAT (1HI)

PRINT 999, LABEL

999 FOFMAT $(1 \times, 7 A 10,15 / /)$

READ ( 1 ) NCHAN, IOREC, DEL TA, NSAMP, FI

L =LENGTH (1)

FEAD I I HCHAN,IDREC D DELTA,NSAMP.HI $L=L E N G T H($ I)

FE AD ( 1 )

C IF (EOF ( 1 ).EQ.0. STOP 1

INE WVE HEIGHT

$N=1$

I $=$

IF (HIII) $\bullet$ L. T. O.) 404,403

$403.1=1+10$

GO TO 40

402 IF (HI(I) $\bullet$ GT $, 0.1416,404$

$404 I=I+1$

GO TO 402

$416-1=1$

425 HMAX(N)=0。 $I=1 \leftarrow 7$

IF (MILI) GT O.) 415.404

415 IF (HI II) GT HMAX (N) ) 407,406

407 MMAX(N) =HI(I)

$X T 1(N)=1$

$406 \quad I=I+I$

$I F(H I(I), G T \cdot 0,1415,430$

43060 TO $(431,413), N$

$431 \operatorname{MMIN}(N)=0$.

$I=I+7$

41 IF (HIII) ITEHMIN(N) 432,410

$432 H M I N(N)=H I(I)$

$410 I=I+I$

IF (HI (I) L T.0.) 411,433

$+33-\mathrm{N}=\mathrm{N}+1$

GO TO 425 


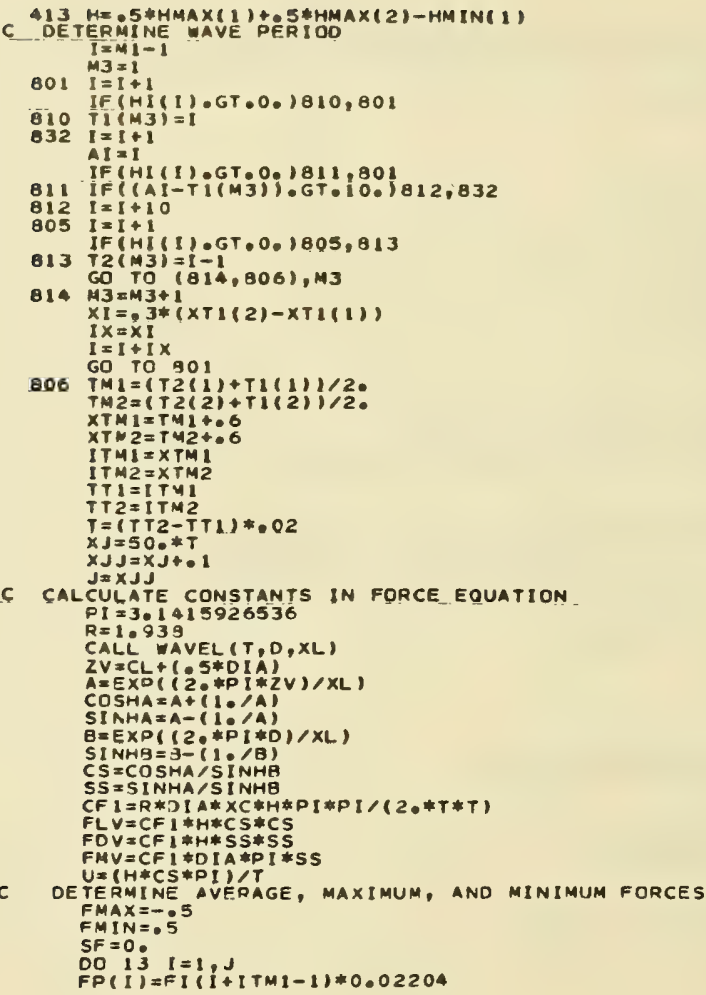


IF (FP(I).GT.FMAX)902,90

902 FMAXXFP(B)

901 IF (FP(I):LT,FMIN) 903,12

903 FMIN=FP(I)

$125 F=S F+F P$ (I)

13 CONTINUE

C CALCULATE SUMS OF SOUARES AND PRODUCTS

SFF $=0$.

$S F X=0$

$S F Y=0$.

$\mathrm{SF} Q \mathrm{O}=0$

SF $Z=0$

DT $=3.1415926536 /(25 \cdot T)$

$A=-D T$

Do $15,1=1, J$

$\mathbf{A}=\mathbf{A}+\mathbf{D T}$

$x(1)=\cos (A)$

$\mathrm{B}=2 \cdot * \mathrm{~A}$

$Y(I)=\cos (B)$

Q(I) ISIN(B)

$C=\operatorname{SIN}(A)$

Z( $)=C * A B S$ (C)

$S F F=S F F+F$ (I ) *F (I

$S F X=S F X+F(1)+C X(I)$

$S F Y=S F Y+F(I)+Y(I)$

SF $=2 F Y+F, 1, \% O(1)$

$S F Q=S F Q+F(I) * O(I)$
$S F Z=S F Z+F(I) * Z(I)$

15 CCNTINUE

$5 X X=-5 * x$

$5 X X=-5 * x$

$500=5 * x y$

$S Z Z=375 \div x$

$A X=S F X / S X X$

$A Y=S F Y / S Y Y$

$A Q=5 F O / S O O$

$A Z=5 F Z / S Z Z$

$\forall X=A X * S F X$

$Y Y=A Y+S F Y$

$Y Q=A O \# S F O$

$Y Z=A Z$ SF Z

YR $=5 F F-V X-V Y-V Z-V O$

C CALCULATE COEFFICIENTS AND PARAMETERS PHI ANO $K$ PHI $=28.64789 * A T A N 2(40, A Y)$

IF (PHI L T $-45 \bullet) 7999,8999$

7999 PNI $=$ PHI + 190 .

0090 CCNTINUE

$Y A=S O R T$ ( $\triangle O * A O+A Y \# A Y$ )

$C L V=2 * Y A / F L V$

$A N G=(A N G L E * P I) / 180$.

$C L \vee A=C L V / C O S$ ( $A N G$ )

$C L \vee U=C L \vee A / C O S(A N G)$

$C M V=-A X / F M V$

CDV $=-A Z / F D V$

$X K=-5-(S F,(C L V * F L V))$ 
PR INT 4

4 FOAMAT (10X,6HT (SEC), 7X, 6HHT (FT), 5X, 9HWAVEL (FT), 6X, THDEP(FT), 4 X, 9HU

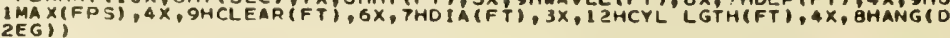

PRINT $300, T, H, X L, D, U, C L, D I A, X C, A N G L E$

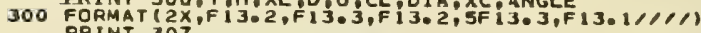
PRINT 307

307 FORMAT $(6 X, 12 H T O T A L$ SUM SO, 6X, 5HCOS2A, $10 X, 5 H S I N 2 A, 11 X, 4 H C O S A, 8 X, 1$ OH ISINA/SINA/, 6X, BHVAR I ANCE

FRINT 301, SFF, VY, VO, VX, VZ, VR

301 FORMAT $(6 F 15.6 \%)$

FRINT 310

310 FCRMAT $(26 X, 2 H A Y, 13 X, 2 H A Q, 13 X, 2 H A X, 13 X, 2 H A Z, 13 X, 2 H Y A)$

302 PRINT $302, A Y, A O, A X, A Z, Y A$

302 FOAMAT $(15 X, 5 F, 5: 6$,

300 FOFMAT (4IX, 3HFLV, 12X, 3HFMV, $12 X, 3 H F O V)$

PRINT $303, F L V, F M V, F D V$

303 FORMAT $30 X, 3 F$ I $5.6 \% 1 / 1)$ FRINT 104

104 FORMAT $110 X, 4 H$ CLY,10X,5H CLVA,10X,5H CLVU,11X,5H CMY, 10X,5H CDV, $111 \times, 2 \mathrm{H} \times, 12 \mathrm{X}, 4 \mathrm{H}$ PHI

305 FORMAT 05 , CLV, CLVA, CLVU, CMV, COV, XK, PHI

305 FORMAT $(5 F 15,3, F 15.4, F 15.211 \% 1)$

309 PRINT 309

309 FOAMAT $(38 \times$, BMFAVGILB),

30 FORMAT ( $30 X, F i 5.6,2 F 15.5 / 1 / 1)$

PUNCH $387, C L, D I A, A N G L E, T, H, X L, U, C L Y, C L Y A, C L V U, P H I, X K, C M Y, C D V$

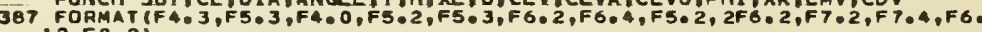
$12, \mathrm{FB}, 2$

C PLCT ORIGINAL DATA ANO RESULTS FOR COMPARISON

(60) IF IT.GT.1.8)601.600

(T.GT.2.4) 603,602

$600 \mathrm{JS}=2$

60 To 606

602 is $=3$

$603 \mathrm{JS}=4$

606 C=ABS (FMAX)

$A=A B S$ (FMIN)

$B=A M A X 1(A, C)$

$\mathrm{BB}=35, \mathrm{~B}$

$C C=300 /$ HMAX ( 1$)$

PRINT 26

26 FORMAT $(4 X, 7 H$ FP(L日), 3X,7H FV(L日), $2 X, 8 H$ RES (LB)/

DO $31 L=1,101$

$G(L)=14$

31 CONTINUE

DO $32 \quad I=1, J, J S$

G(5) $=1 \mathrm{H}$

MP $(1)=H I(I+1 T M 1-1)$

$F V(I)=A X * X(I)+A O * O(I)+A Y * Y(I)+A Z * Z(I)+S F$

RES $(I)=F P(I)-F V(I)$

$J J=51$ * $+B Q F P(I)$

$K K=51 \cdot+8 B+F V(1)$

$4 L=510+8$ Q

GA $=51, \pm+C C$

$G(K K)=1 H 4$

G(Lㄴㄴ) $=1 \mathrm{H}$

$G(M M)=1 H$

PRINT 100,FP(I) FVEI) RES (I), G

100

FOAMAT (IHZ, 3F $10.5,101 A 1 /$

$G(7)=14$

$G(K K)=1 H$

$G(L ᄂ)=1$ H

$G(M M)=1 H$

32

CONTINUE

GO To 8

10 CONTINUE

END

SUBROUTINE VAVEL $(T, D, X L)$

E=32.2\%T/ 3.283185

$T P D=6,283185 \% 0$

IF (B-TOD) $2,2,3$

c IEEP VATER INITIAL ESTIMATE FOR WAVELENGTH

$2 \quad x L=8$

CO TO

c

SMLLOU

$3 \quad X L=T=X L$

$X L=\theta * T A N H(T P D / X L X)$

IF (ABS $(X L X-X L)-0.05)=, 4,4$

5 RETUFN

END 
APPENDIX D

COMPUTER PROGRAM FOR HORIZONTAL LEAST SQUARES ANALYSIS (TWO-DIMENSIONAL DATA) 
PROGRAM MORIZL (INPUT, OUTPUT, PUNCH)

DI HENS I ONZ(41),P(41), YS (41), YI(41),FI(41),FP(41),F(41),FH(4I), IRES $(41), \mathrm{G}(101)$

C SET TEST CENOITIONS

DI $A=0.333$

$X C=0.917$

ANGLE $=0$.

$D=2 \bullet C 00$

C READ IN DIGITIZED DATA

8 READ , UP, DN, CFD, CFU

9 READ $2, C L, T, N, X w, X F, C, W O, F O$

READ 3, (YI (I),FI (I), I $=1,40$ )

FORMAT (2F3, 3, 2F 3.0)

FOFMAT (F 3, 3, F3,2, I $2,2 F 2,1,3 F 3 \bullet 0)$

2 FOFMAT ( $F$ B 3 , F

C DETERMINE WAVE HEIGHT

DO $1=1,31,10$

IF $(V 1$ I I $3-10) 21,23,23$

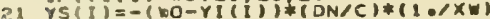

GO TO 11

$23 Y S(I)=(Y I(I)-\forall O) *(U P / C) *(1, / X)$

I I CONTINUE

$M=(Y S(1)+Y S(21)-Y S(11)-Y S(31)) / 2$

C CALCULATE CONSTANTS IN FORCE EQUATION

$P I=3.1415926536$

$F=1.938$

CALL WAVELIT,D,XL)

ZV $=C L+1.5 * 01 A)$

$A=E X P(2 * P I * Z V) / X L)$

COSHA $=A+(10 / A)$

$B=E X P((2 * * P I \div 0) / X L)$

SI $\mathrm{NHB}=\mathrm{B}-(1 . / \mathrm{B})$

$\mathrm{CS}=\mathrm{COSHA} / \mathrm{S}$ INHB

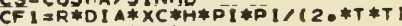

$F O H=C F 1 * H * C S * C S$

$F M H=C F I * 0 I A * P I * C S$

C DETERMINE AVERAGE, MAXIMUM, AND MINIMUM FORCES

FMAX $=-\circ 5$

FMIN=. 5

$\mathrm{SF}=\mathrm{Q}$.

DO $13 \quad I=1,40$

IF (FI I I ) -FO) 70 I 702,702

701 FPII $=(F O-F I(I) *(C F O / C) * 0.02204 / \times F$

Go 7003

702 FP(I I =-(FI (I)-FO)*(CFU/C)*.002204/XF

703 CONTINUE

IF (FP(I), GT FMAX)902,901

902 FMAX $=F P(1)$

901 IF(FP(I):LT.FMIN)903,12

903 FMIN=FD

$12 S F=S F+F P(I)$

13 CONTINUE

C CAlCulate SUMS OF SGUARES AND PRODUCTS 


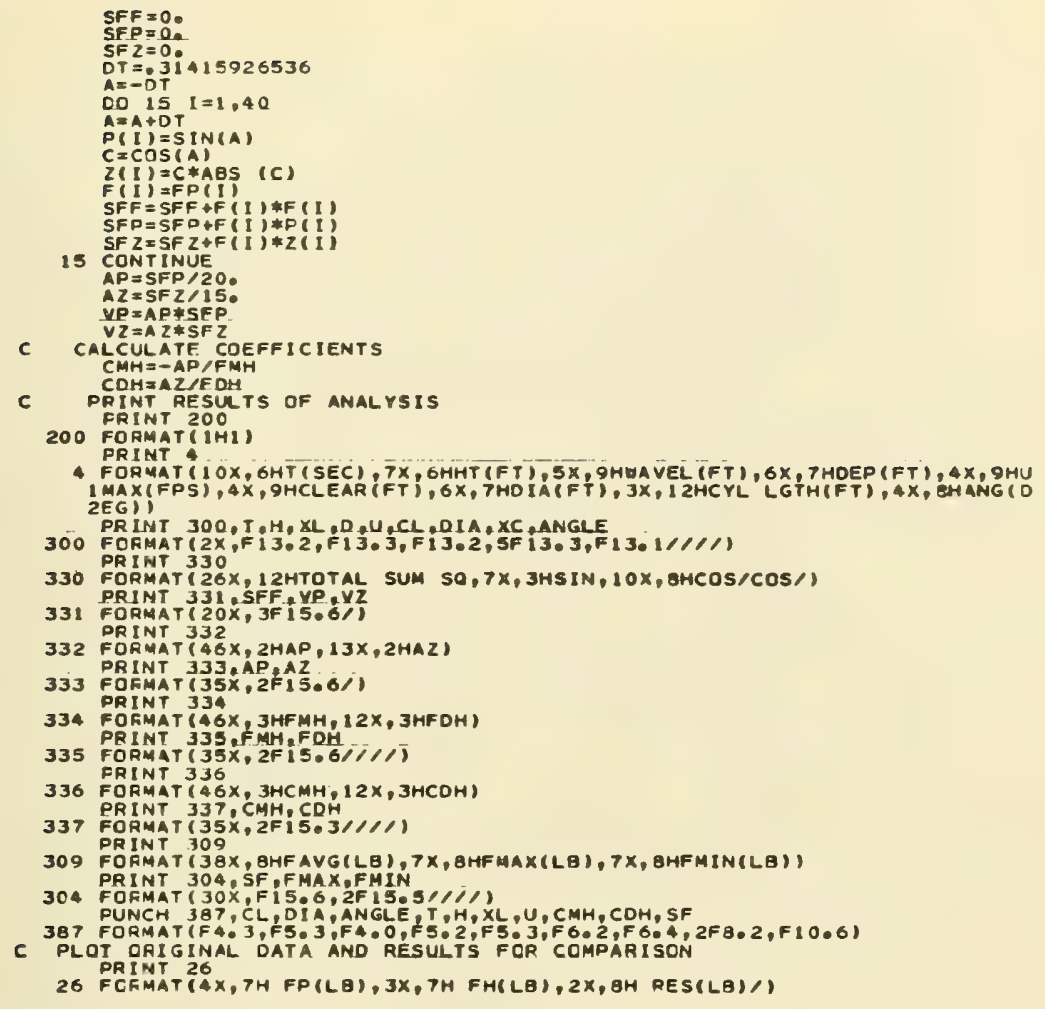




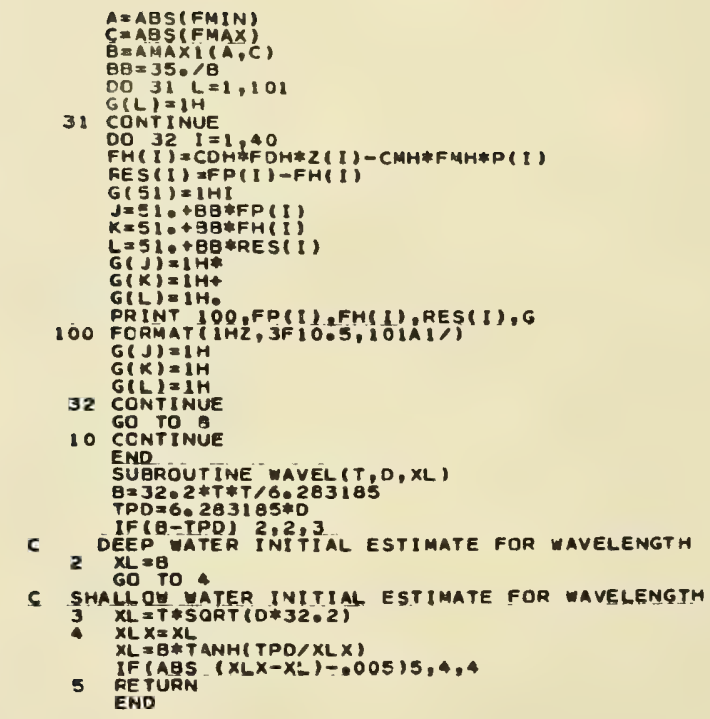


APPENDIX E

TABULATED VERTICAL FORCE DATA FROM TWO-DIMENSIONAL EXPERIMENTS 


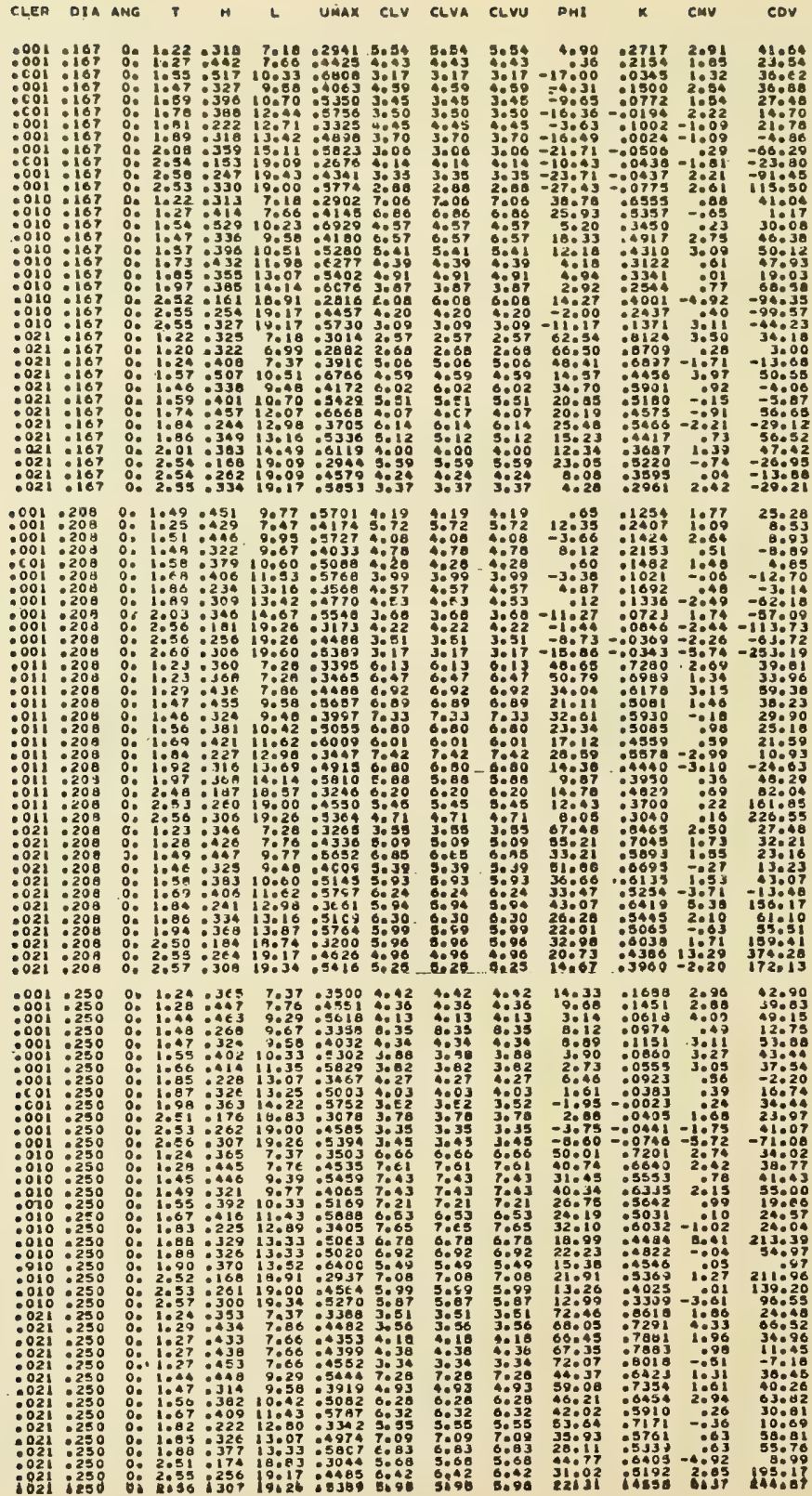




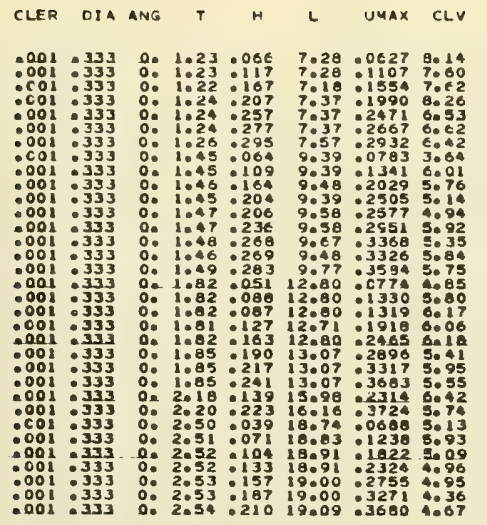

\begin{tabular}{|c|c|c|}
\hline CLVA & CLVU & PHI \\
\hline 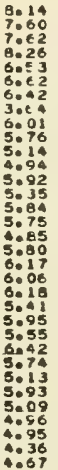 & 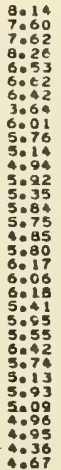 & 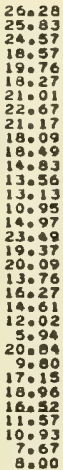 \\
\hline
\end{tabular}

K CMV COV

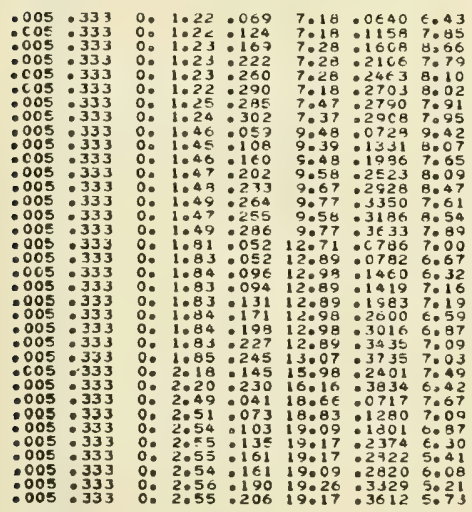

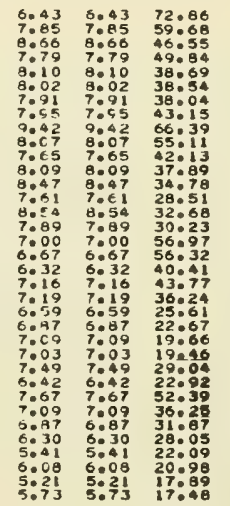

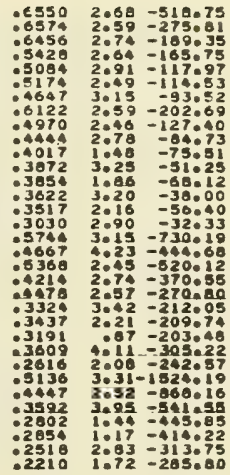

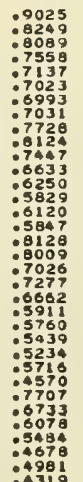

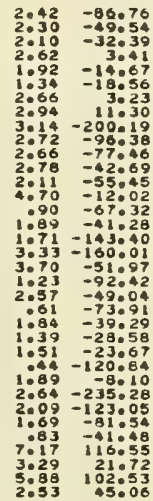

.010 .353

$0.1 .29 .0507 .29 \cdot \cos 33.90$

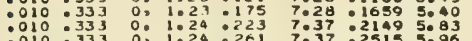

$\because: 10: 333 \quad 0: 1: 24: 261 \quad 7: 37: 25155096$

$\because 010: 331$ 0: $1: 23: 281 \quad 7: 28: 268860470$

$: 010: 333 \quad 0: 1.49: 061 \quad 9.67: 07735022$

$: 010: 333 \quad 0,1: 48: 109 \quad 9.67: 1996 \quad 6025$

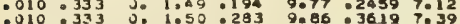

$: 010: 333 \quad 0.1050: 283 \quad 9=86: 36197039$

$: 010: 333 \quad 0,1: 47: 239 \quad 9077: 30148: 78$

$: 010: 3330: 1,43: 267 \quad 9.67: 33597: 23$

$: 010: 333$ 0: $1.80: 051 \quad 12.62: 076060.40$

$: 010: 333$ oj $1.83: 13312.89: 20136.43$

$010: 333 \quad 0: 1: 83: 20212.89: 30437: 08$

$: 010: 333 \quad 0: 1: 97: 227 \quad 13025: 34937001$

$\because 010: 333 \quad 0 ; 2.21: 23516.25: 39246099$

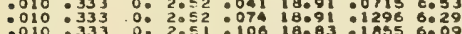

$: 010: 333 \quad 0: 2.54: 1401900: 2458012$

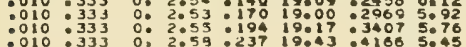

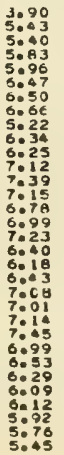

5.90

$2.33 \quad-92.55$

$\begin{array}{lllll}5.40 & 74.26 & : 9467 & 2.36 & -37.36 \\ 35.03 & 9194 & 2.40 & -25: .68\end{array}$

$5.83 \quad 65.11 \quad 0.8594 \quad 2039 \quad-22.32$

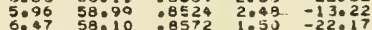

$6: 50 \quad 59: 66 \quad: 8167 \quad 3: 31 \quad-4: 04$

$\begin{array}{lllll}6.66 & 56.77 & .7820 & 2.77 & -10.84\end{array}$

$\begin{array}{lllll}5.22 & 04.19 & 09752 & 2.35 & -103 \\ 6.34 & 73.27 & 08764 & 2.45 & -61: 69\end{array}$

$\begin{array}{lllll}6.25 & 63.87 & 8543 & 2.46 & -35.52 \\ 7.12 & 58.19 & 7879 & 3.02 & -0.112\end{array}$

$\begin{array}{lllll}7.12 & 58.19 & 07879 & 3.02 & -9.12\end{array}$

$\begin{array}{lllll}7.39 & 46: 01 & : 7367 & 1.54 & -27.97 \\ 7.15 & 43.14 & 7609 & 2.38 & -32.91\end{array}$

$\begin{array}{lllll}6.99 & 540.01 & : 7776 & 2.50 & -8.64 \\ 7.03 & 3.47 & 13 . t & 1\end{array}$

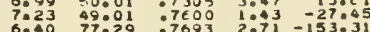

$6.18 \quad 64: 55: 8601 \quad 3: 35-58: 73$

$\begin{array}{lllll}6: 43 & 57: 07 & : 8120 & 1: 92 & -62.83 \\ 7009 & 39.13 & 7109 & 053 & -31.74\end{array}$

$\begin{array}{lllll}7: 09 & 39: 13 & : 7109 & 065 & -31: 74\end{array}$

$7.14 \quad 36.34 \quad 6430 \quad 1.21 \quad 10004$

$6.9928 .44: 5873 \quad 1.55 \quad 3.03$

0.29 55.75 8014 $300-260.20$

$\begin{array}{lllll}8.29 & 55.75 & 0014 & 3.46 & -66.29 \\ 8.09 & 44.332 & 7355 & 4.52 & -30.30\end{array}$

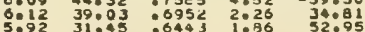

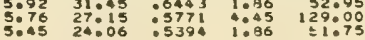




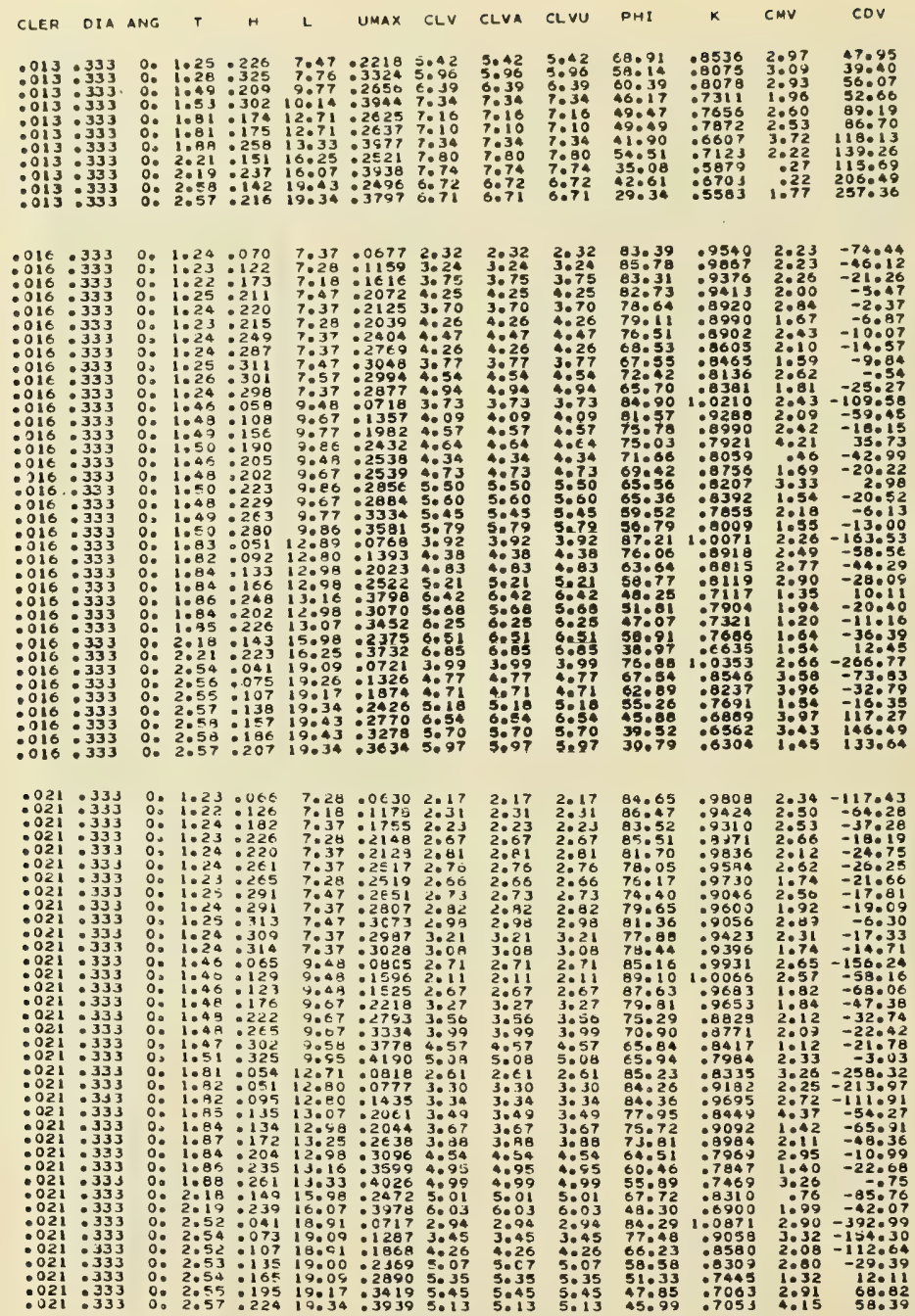




\begin{tabular}{|c|c|c|c|c|c|c|c|c|c|c|c|c|c|}
\hline$E R$ & $D \& A$ & IG & $T$ & $\mathrm{H}$ & L & $A x$ & LV & LVA & Lve & PHI & $k$ & MV & DV \\
\hline $\begin{array}{l}083 \\
083 \\
083 \\
083 \\
083 \\
083 \\
083 \\
083 \\
083 \\
083 \\
083 \\
083 \\
083 \\
083 \\
083 \\
083 \\
083 \\
083 \\
083 \\
083 \\
083 \\
083 \\
083 \\
083 \\
083 \\
083 \\
083 \\
083 \\
083 \\
083\end{array}$ & 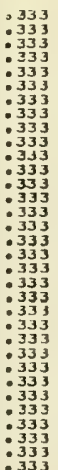 & 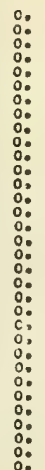 & 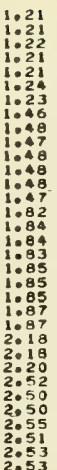 & 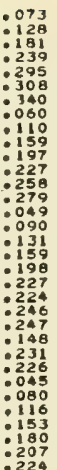 & 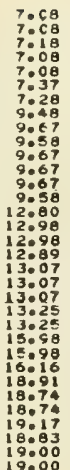 & 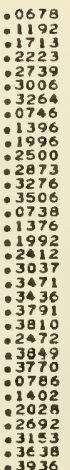 & $:$ & $\begin{array}{l}\text { : } \\
: 4 \\
\text { : } \\
: 8 \\
: 5 \\
: 5 \\
: 8 \\
: 1 \\
: 8 \\
: 7 \\
: 7 \\
: 8 \\
: 7 \\
: 8\end{array}$ & $\begin{array}{r}1: 03 \\
1: 30 \\
: 47 \\
: 97 \\
: 99 \\
1: 00 \\
1: 18\end{array}$ & $\begin{array}{l}89.63 \\
79.84 \\
78.84 \\
81.48 \\
92.66 \\
83.98 \\
86.58 \\
83.31 \\
84.57 \\
77.25 \\
74.50\end{array}$ & 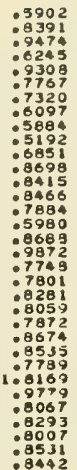 & $\begin{array}{l}: 92 \\
1: 67 \\
1: 87 \\
1: 25 \\
1: 52 \\
1: 22 \\
1: 51 \\
2: 04 \\
1: 82 \\
1: 62 \\
1: 29 \\
1: 29 \\
: 99 \\
088\end{array}$ & $\begin{array}{r}-44.23 \\
-28.81 \\
-16.33 \\
1.70 \\
-4.82 \\
-2.90 \\
-76.74 \\
-34.11 \\
-29.26 \\
-13.82 \\
-14.65 \\
-9.46\end{array}$ \\
\hline
\end{tabular}

\begin{tabular}{|c|c|c|c|c|c|c|c|c|c|c|c|c|}
\hline $\begin{array}{l}.167 \\
1167 \\
1167 \\
1167 \\
167 \\
167 \\
167 \\
167 \\
167 \\
167 \\
167 \\
167 \\
167 \\
167 \\
167 \\
167 \\
167 \\
167 \\
167 \\
167 \\
167 \\
1657 \\
1167 \\
167 \\
167 \\
167 \\
167 \\
167 \\
167 \\
167 \\
167 \\
167 \\
167 \\
167 \\
167 \\
167 \\
167 \\
167 \\
167\end{array}$ & 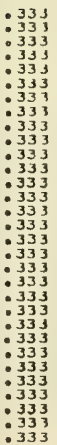 & 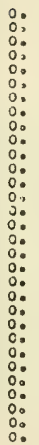 & 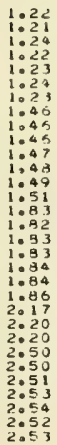 & 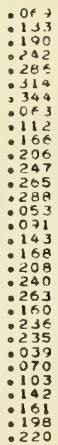 & $\begin{array}{l}7.16 \\
7008 \\
7037 \\
7018 \\
7028 \\
7037 \\
7.28 \\
9.48 \\
9048 \\
9048 \\
3058 \\
9.67 \\
9.77 \\
9.95 \\
12.89 \\
12.90 \\
12.89 \\
12.89 \\
12078 \\
12.98 \\
13.16 \\
15.90 \\
16.16 \\
16.16 \\
18.74 \\
18.74 \\
18.83 \\
19.00 \\
19.09 \\
14091 \\
19.00\end{array}$ & 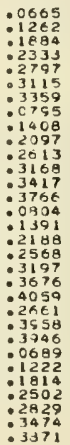 & 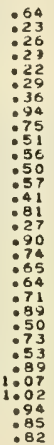 & 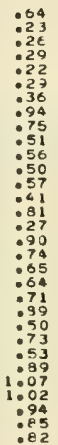 & $\begin{array}{l}.64 \\
: 23 \\
: 26 \\
: 29 \\
: 22 \\
: 29 \\
: 36 \\
: 94 \\
: 75 \\
: 51 \\
: 55 \\
: 50 \\
: 57 \\
: 41 \\
: 81 \\
: 27 \\
: 90 \\
: 74 \\
: 65 \\
: 64 \\
: 71 \\
: 89 \\
: 50 \\
: 73 \\
: 53 \\
: 89 \\
: 07 \\
: 02 \\
: 94 \\
: 85 \\
082\end{array}$ & $\begin{array}{r}133.85 \\
126: 26 \\
52.10 \\
87.88 \\
90.47 \\
51.93 \\
99.40 \\
80.63 \\
92.43 \\
95.83 \\
87.03 \\
90.62 \\
97.84 \\
87.71 \\
86.40 \\
119.51 \\
99.79 \\
104.78 \\
94.40 \\
88.35 \\
90.47 \\
72.48 \\
76.68 \\
79.10 \\
95.87 \\
84.47 \\
89.60 \\
93.80 \\
94.19 \\
96.58 \\
98.07\end{array}$ & $\begin{array}{r}0404 \\
1: 3436 \\
: 7354 \\
: 2581 \\
: 2342 \\
: 0740 \\
-2479 \\
: 1263 \\
: 3327 \\
: 5207 \\
: 5324 \\
: 5018 \\
: 551 \\
: 4472 \\
: 8714 \\
: 5701 \\
: 381 \\
: 5671 \\
: 5719 \\
: 5226 \\
: 5391 \\
: 5973 \\
: 5292 \\
10978 \\
: 5163 \\
: 5544 \\
: 5859 \\
: 5919\end{array}$ & $\begin{array}{rr}1.91 & -12.43 \\
1.83 & -12.89 \\
1: 78 & -8.56 \\
1.35 & -3.96 \\
1.75 & -6.29 \\
1: 71 & -.88 \\
1: 76 & -.83 \\
1.86 & -36.17 \\
1.83 & -15.99 \\
1.80 & -13.60 \\
1.69 & -7.57 \\
1.56 & -6.24 \\
1: 56 & -4.50 \\
1: 32 & -3.25 \\
1: 85 & -54.59 \\
1.81 & -23.46 \\
1.50 & -15.99\end{array}$ \\
\hline
\end{tabular}


APPENDIX F

TABULATED VERTICAL FORCE DATA FROM THREE-DIMENS IONAL EXPERIMENTS 


\begin{tabular}{|c|c|c|c|c|c|c|c|c|c|c|c|c|c|}
\hline & I 4 & $N C_{3}$ & $T$ & $\mathbf{H}$ & L & $\operatorname{Aax}$ & ELV & CLVA & LVU & PII & $\mathbf{k}$ & $M V$ & DV \\
\hline 1 & 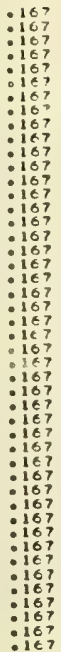 & $\begin{array}{l}0: \\
0: \\
0: \\
0: \\
0: \\
0: \\
0: \\
0: \\
0: \\
0: \\
0: \\
0: \\
0: \\
0: \\
0: \\
30 \\
30 \\
30 \\
30 \\
30: \\
30: \\
30: \\
30: \\
30: \\
30:\end{array}$ & $\begin{array}{l}2.24 \\
1: 74 \\
1.31 \\
1.20 \\
2.32 \\
2.06 \\
1: 00 \\
1.32 \\
2.40 \\
2.24 \\
1.98\end{array}$ & 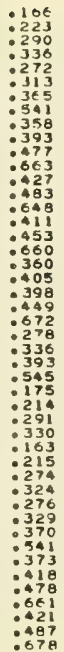 & 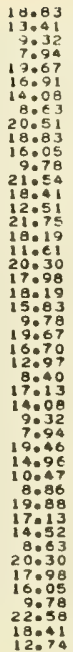 & 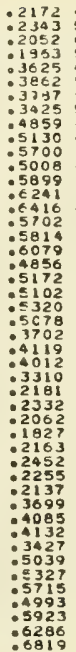 & 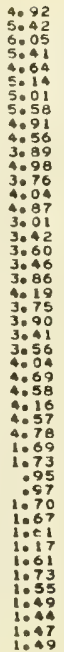 & $\begin{array}{l}1 \\
5 \\
1 \\
5 \\
0 \\
7 \\
7 \\
8\end{array}$ & 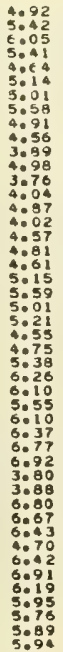 & $\begin{array}{r}5 \\
2 \\
7 \\
9 \\
-16 \\
-17 \\
-17 \\
-8 \\
1 \\
-29 \\
=32 \\
-29 \\
-7 \\
-37 \\
-37 \\
-20 \\
-35 \\
-31 \\
-12 \\
-19 \\
-24 \\
-21 \\
-21 \\
-3 \\
-9 \\
-12 \\
-1\end{array}$ & $\begin{array}{r} \\
\therefore \\
\therefore \\
\therefore \\
\because \\
- \\
\therefore\end{array}$ & $\begin{array}{l}4 \\
2 \\
3 \\
5 \\
2 \\
5 \\
21 \\
5 \\
3 \\
5 \\
16 \\
8\end{array}$ & $\begin{array}{r}-252.67 \\
-211.71 \\
-128.34 \\
-87.75 \\
-398.58 \\
-184.41 \\
-74.05 \\
-32.56 \\
-69.11 \\
-389.23 \\
-88.67 \\
9.63 \\
-262.38 \\
-464.02 \\
-10.62 \\
679.27 \\
213.19 \\
72.50 \\
-43.32 \\
228.36 \\
233.82 \\
60.68 \\
12.23 \\
-91.15 \\
31.98 \\
20.15 \\
15.48 \\
155.65 \\
4.01 \\
-12.52 \\
-34.56 \\
-642.93 \\
-783.71\end{array}$ \\
\hline
\end{tabular}

$: 005: 167 \quad 0,2.56: 392 \quad 22: 177: 5472 \quad 3.58$ $: 005: 167 \quad 0: 1.58: 636 \quad 11.61: 585950.62$ $005: 167 \quad 0: 2.20: 39018.41: 5031: 06$ $-005: 167 \quad 0.1: 95: 456 \quad 15: 83: 5410: 453$ $: 005: 167 \quad 0: 1.42: 639 \quad 9: 78: 4830 \quad 6: 61$ $: 005: 167 \quad 0 ; 2010 ; 30517.34: 39206019$ $\because \cos : 167 \quad 0: 1080: 357 \quad 14: 96: 408050.89$ $: 005: 167 \quad 0: 1: 30: 522 \quad 8.86: 3437 \quad 7: 49$ $.005: 167 \quad 0.1 .96: 201 \quad 15.83 .2397 \quad 7.47$ $: 005: 167 \quad 0: 1: 34: 337,8: 86: 2025 \quad: 15$ $: 005: 16730: 1: 95: 195 \quad 15 . e 3=2315 \quad 6.19$ $.005 \cdot 16730.11 .32 \cdot 311 \quad 8.63 \cdot 19686.26$ $.005 \cdot 16730 \cdot 2.44 \cdot 26620.92 \cdot 36384.36$ $000=1673002.14 \cdot 310 \quad 17.77: 39324.79$ $005: 16730.1098: 36014.96: 41164.85$ $: 005: 16730: 10,14: 546 \quad 9.860: 35995044$ $: 005: 16730: 2.22: 41018.62: 53203.57$ $: \cos : 16730: 1: 42: 643$ 9:78: 4859 5. 33 $005: 16730.2054: 305210605500 \quad 3.25$ - $005: 16730: 2.22: 46318.62: 60123.61$ $: 005: 16730: 1.66: 636 \quad 12.51: 62934.29$ $\because 005: 16750: 2.59: 38922.37 .54542 .13$ $.005 .16760 .1 .64 .632 \quad 12.29 .61502 .11$ $.005: 16760.2 .40 .353 \quad 20.51$. $4792 \quad 2.46$ $\cdot \cos : 16760.2 .22: 40218.62: 52172.40$ $.005 \cdot 16760.2 .00 \cdot 442 \quad 16.26 \cdot 53262.53$ $0005: 167 \quad 60.1146 \quad-432 \quad 10.24: 50571098$ $\because 005: 16760: 2.42: 265 \quad 20.72 \quad 3609 \quad 2.64$ $\because 005: 16760: 2.18: 300 \quad 18: 19: 38 \% 3 \quad 2086$ $005: 16760.1036: 5229.09: 35722,45$ $: 005: 16760: 1: 92: 175$ 15:40:2271 3.29 $: \cos : 16760 ; 1: 66: 23212.51: 22953.60$ $: 005: 16780,1.34: 302$ 8.86 :1791 3.30

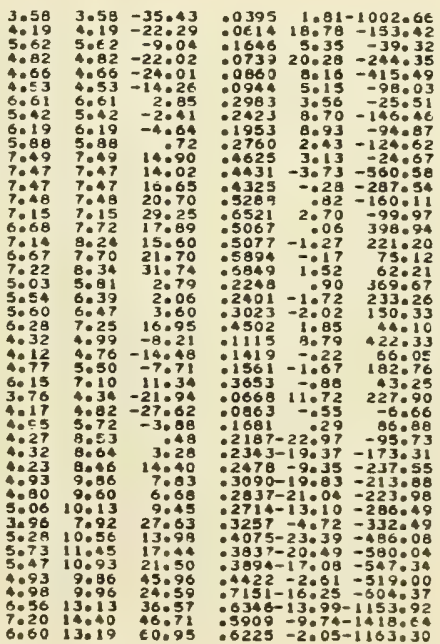


.010 .167 0. 20.7e . 361 24.22.5192 4.30 $: 010: 167,0.2036: 45720.09: 00123.56$ $: 010: 1670: 2.52: 32321: 75: 44765.26$ $\because 10: 167 \quad 0.2 .04: 426 \quad 16.70 \quad-52235.64$ $010: 167 \quad 001.48 \cdot 619$ 10.47:5087 8.39 $-010: 187$ : $0.20: 288$ 18.41:3718 7.96 010.187

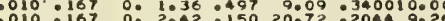
$.010: 167$ 0. $2.06: 18716091: 23089.76$ $: 010: 167$ : $1.76: 22313.64: 23779.49$ $: 010: 16700.1044: 27710.01: 21578040$ $.010 .16730 .2 .38 \cdot 15120.30 .2036 .6039$ $: 010: 167,30: 2.02: 19016.48: 23085.82$ $.010: 16730.1 .42: 283 \quad 9.78: 21385008$ $.010 \cdot 16730,2.46: 24821.13: 34065.61$ $-010: 16730: 2.12: 30417.55: 3026$ E.02 $: 010: 16730: 1: 84: 350 \quad 14: 52: 39106042$ $: 010: 167$ 30: 1.38:504 9.32:3573 5.73

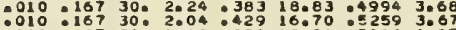
. $010.16730 .1 .46 \cdot 630 \quad 10.24 .50466 .07$ $.010: 16730.2036: 43520.09 .5849 .2072$ .010 .16750 .2065 .38623020 .5474306

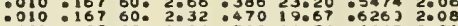
$\div 10.16760 .1 .86 .590 \quad 14074.66632 .94$

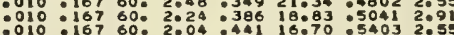
$-010.16760 .1 .46: 64510.24: 51662.20$ $.010 .16760 .2060: 24122.58 \cdot 33642077$ $: 010: 16760.2020: 304 \quad 18.41: 39292.56$ $: 010: 16760$ : $1.38: 498 \quad 9.32: 3531$ 1:91

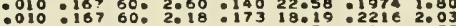
$.10 .16760 .1086 .21414 .74 \cdot 24231.55$

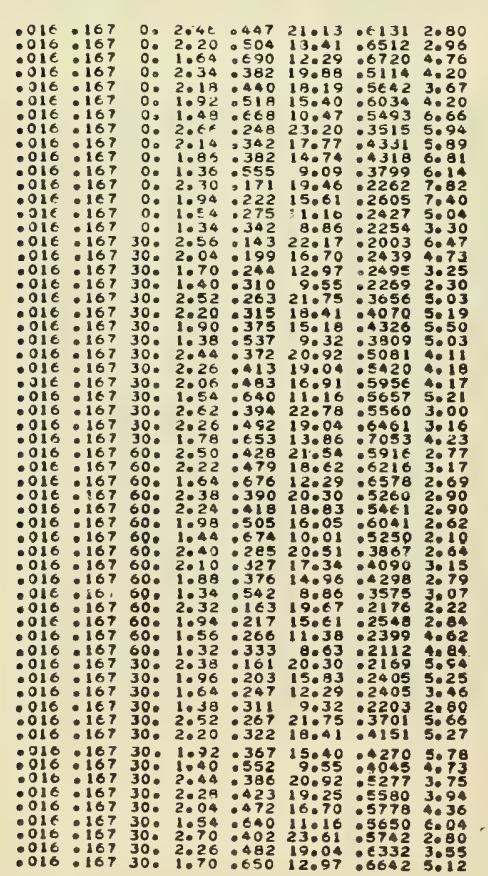

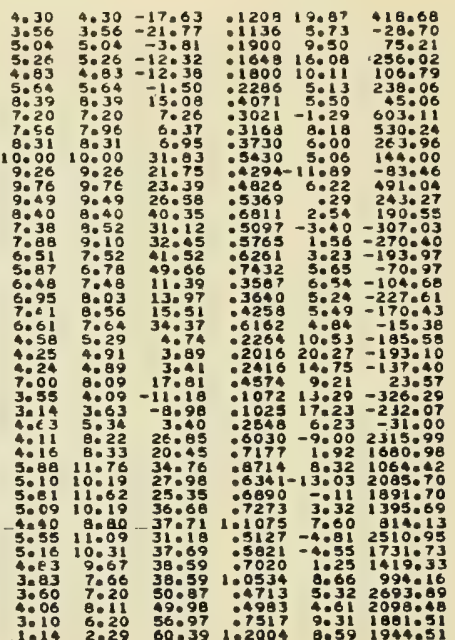

\begin{tabular}{|c|c|c|c|c|c|}
\hline 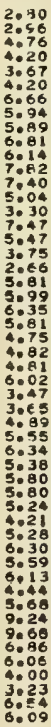 & 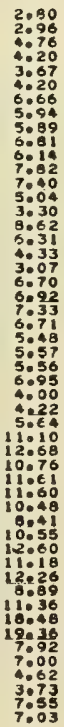 & 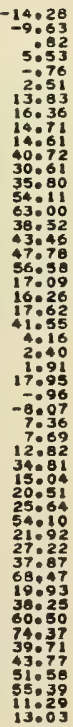 & 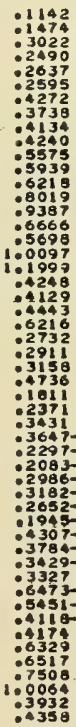 & 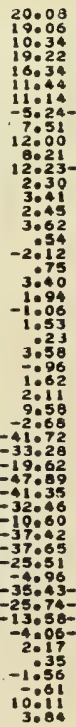 & 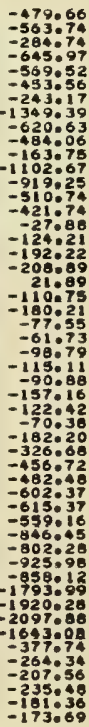 \\
\hline $\begin{array}{l}6.68 \\
5.46 \\
4: 34 \\
5.54 \\
5.03 \\
6.97 \\
3.23 \\
4010 \\
5.91\end{array}$ & $\begin{array}{l}7.71 \\
6: 30 \\
5001 \\
5.25 \\
5: 81 \\
8: 05 \\
3.75 \\
4: 73 \\
6.02\end{array}$ & $\begin{array}{r}19.55 \\
39.77 \\
4.75 \\
1.01 \\
5.27 \\
18.17 \\
-9.97 \\
-2.94 \\
8.37\end{array}$ & 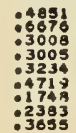 & $\begin{array}{l}5.99 \\
3: 53 \\
5: 22 \\
5010 \\
5.51 \\
1.22 \\
: 60 \\
4: 63 \\
-.97\end{array}$ & $\begin{array}{l}-158.13 \\
-239.03 \\
-113.41 \\
-116.79\end{array}$ \\
\hline
\end{tabular}




\begin{tabular}{|c|c|c|c|c|c|c|c|c|c|c|c|c|c|}
\hline & IA & NNG & $T$ & $M$ & & & & & & PHI & K & Q v & DV \\
\hline $\begin{array}{l}021 \\
021 \\
021 \\
021 \\
021 \\
021 \\
021\end{array}$ & $\begin{array}{l}167 \\
1167 \\
1167 \\
1167 \\
1167 \\
167 \\
167 \\
167 \\
167 \\
1167 \\
1167 \\
1167 \\
1167 \\
1167 \\
1167 \\
167 \\
167 \\
167 \\
167 \\
167 \\
167 \\
167 \\
167 \\
167\end{array}$ & $\begin{array}{l}0 . \\
0: \\
0: \\
0: \\
0: \\
0: \\
0: \\
0 \\
0: \\
0 \\
0: \\
0: \\
30 \\
30 \\
30 \\
30 \\
30 \\
30 \\
30 \\
30 \\
30 \\
30 \\
30 \\
30 \\
30 \\
30 \\
30 \\
30 \\
60 \\
60\end{array}$ & $\begin{array}{l}2.40 \\
2: 119 \\
1.56 \\
2.59 \\
2.24 \\
1: 92 \\
1.40 \\
2.50 \\
2.32 \\
2.04 \\
1.52 \\
2.68 \\
2.38 \\
1.80 \\
2.78 \\
2.70 \\
2.36 \\
1.84 \\
2.48\end{array}$ & 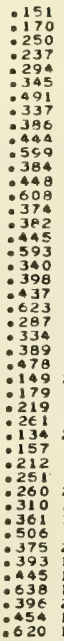 & $\begin{array}{l}22 \\
19 \\
140 \\
110 \\
210 \\
180 \\
150 \\
20\end{array}$ & 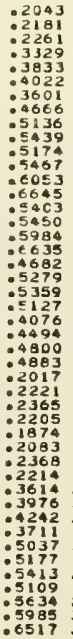 & 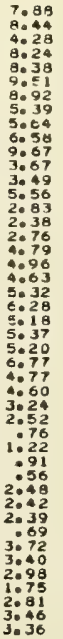 & & $\frac{7}{5}$ & $\begin{array}{r}38 \\
39 \\
52 \\
11 \\
17 \\
22 \\
40 \\
1 \\
5 \\
7 \\
20 \\
-7 \\
-4 \\
8 \\
1 \\
8 \\
11 \\
12 \\
13 \\
13 \\
14 \\
19\end{array}$ & $\begin{array}{l}5 \\
1 \\
4 \\
2 \\
0 \\
9 \\
0 \\
9 \\
9 \\
6 \\
9 \\
9 \\
5 \\
9 \\
0 \\
5 \\
6 \\
5 \\
9 \\
7 \\
0 \\
0 \\
8 \\
4 \\
0 \\
2 \\
1\end{array}$ & $\begin{array}{r}2 \\
3 \\
3 \\
3 \\
6 \\
7 \\
2 \\
16 \\
13 \\
5 \\
8 \\
16 \\
16 \\
9 \\
31 \\
20 \\
19 \\
11 \\
19 \\
19 \\
19 \\
10 \\
15 \\
12 \\
10 \\
4\end{array}$ & $\begin{array}{r}37 \\
20 \\
14 \\
39 \\
25 \\
9 \\
-2 \\
2 \\
4 \\
-13 \\
-2 \\
-63 \\
-44 \\
-42 \\
-20\end{array}$ \\
\hline
\end{tabular}

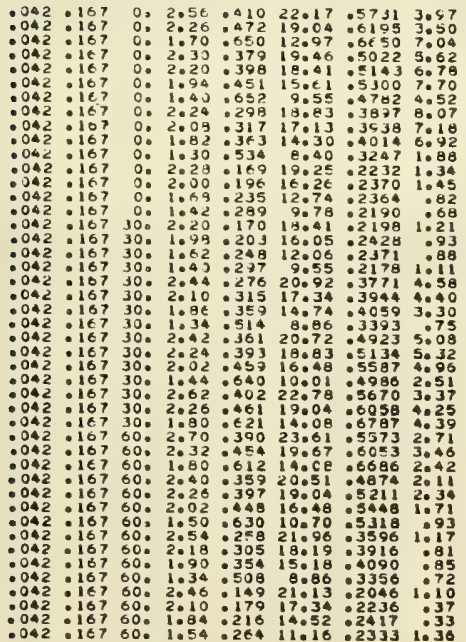

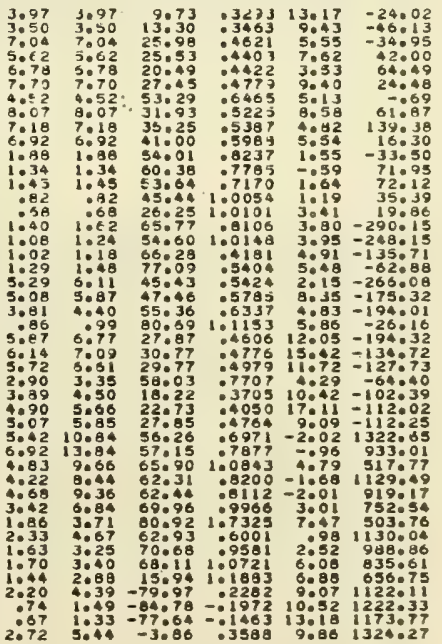




\begin{tabular}{|c|c|c|c|c|c|c|c|c|c|c|c|c|c|}
\hline & CIA & NF & $T$ & H & L & UMAX & LLV & LVA & LVu & PHI & K & MV & CDV \\
\hline 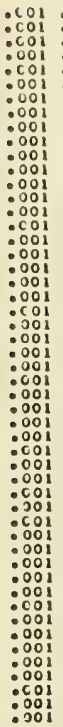 & 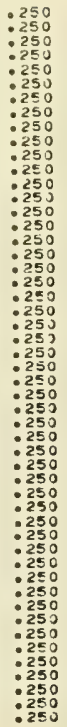 & 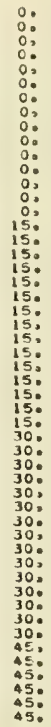 & 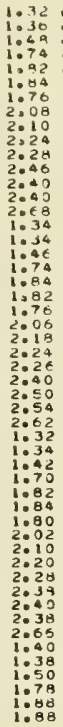 & 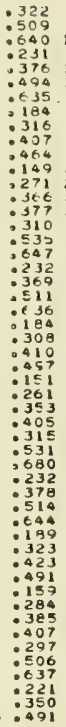 & $\begin{array}{r}9.63 \\
7009 \\
10.47 \\
13.41 \\
14.30 \\
14.52 \\
13.64 \\
17.13 \\
17.34 \\
18.83 \\
19.25 \\
21.13 \\
20.51 \\
23.51 \\
23.40 \\
9.86 \\
3.86 \\
10.24 \\
13.41 \\
14052 \\
14.30 \\
13.64 \\
19.91 \\
19.19 \\
19.93 \\
19.04 \\
20.51 \\
21.54 \\
21.96 \\
22.78 \\
8.63 \\
8.86 \\
9.78 \\
12.97 \\
14030 \\
14.52 \\
14.09 \\
15.49 \\
17.34 \\
12.41 \\
19.25 \\
20.30 \\
20.51 \\
20.30 \\
23.20 \\
3.55 \\
3.32 \\
10.70 \\
13.86 \\
14.96 \\
14.96\end{array}$ & 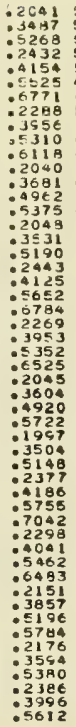 & $\begin{array}{l}5021 \\
5034 \\
5003 \\
4086 \\
4037 \\
5008 \\
5025 \\
4022 \\
4090 \\
5005 \\
4069 \\
4005 \\
4048 \\
5017 \\
4093 \\
4083 \\
4068 \\
4054 \\
4031 \\
4077 \\
4076 \\
4038 \\
3083 \\
4000 \\
4064 \\
4007 \\
4004 \\
4003 \\
045 \\
4011 \\
3095 \\
3071 \\
3069 \\
3037 \\
3043 \\
4019 \\
3078 \\
3043 \\
3020 \\
3064 \\
3033 \\
2094 \\
2090 \\
2062 \\
2073 \\
2058 \\
2038\end{array}$ & $\begin{array}{l}5 \\
5 \\
5\end{array}$ & $\begin{array}{l}5 \\
5 \\
5 \\
5 \\
5 \\
5 \\
5\end{array}$ & 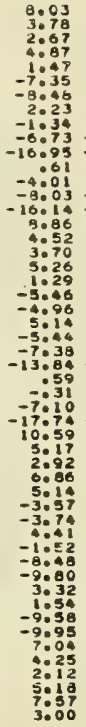 & 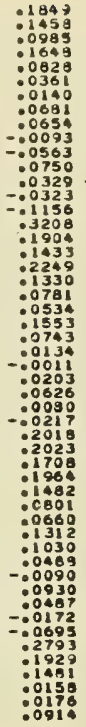 & $\begin{array}{r}3.90 \\
4.72 \\
-0.09 \\
3.13 \\
2.54 \\
5.18 \\
2.31 \\
2.53 \\
4.56 \\
2.08 \\
7.35 \\
1.56 \\
-3.39 \\
1.91 \\
3.37 \\
2.70 \\
4.79\end{array}$ & 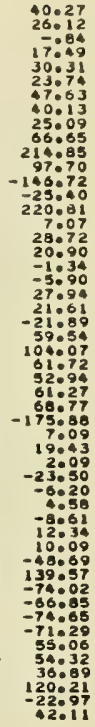 \\
\hline
\end{tabular}
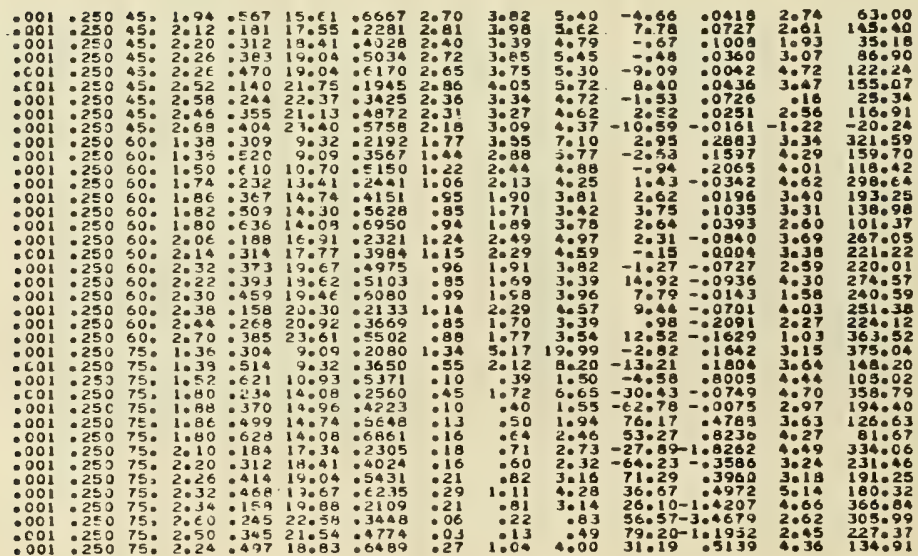


\begin{tabular}{|c|c|c|c|c|c|c|c|c|c|c|c|c|c|}
\hline & 12 & N.; & $T$ & $M$ & L & YMAX & CLV & CLVA & LVU & $\mathrm{HI}$ & $k$ & CMV & $=0$ \\
\hline 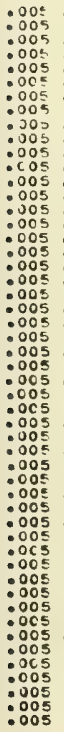 & 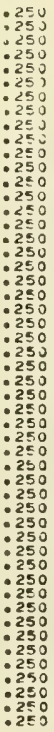 & 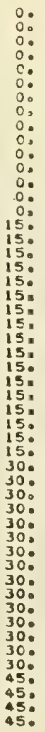 & 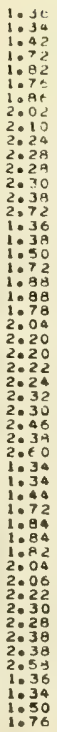 & 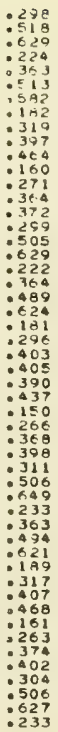 & 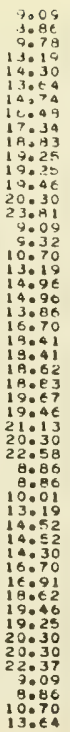 & 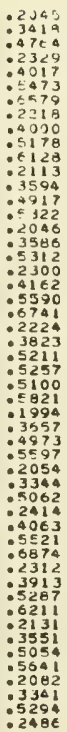 & 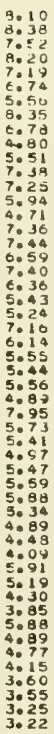 & $\begin{array}{l}9: 1 \\
9: 3 \\
7.5\end{array}$ & $\begin{array}{l}10 \\
139 \\
: 52 \\
.20 \\
119 \\
774 \\
55 \\
135 \\
78 \\
80 \\
51 \\
118 \\
125 \\
79 \\
711 \\
89\end{array}$ & 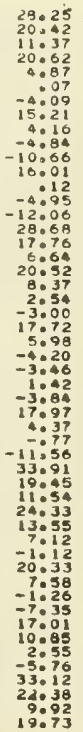 & 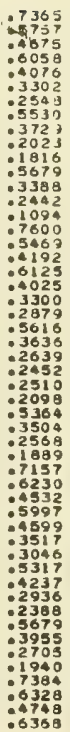 & 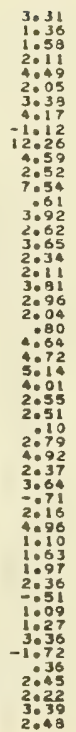 & 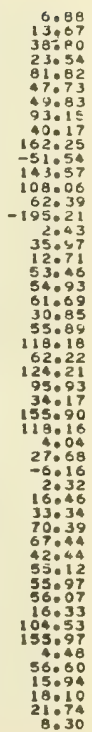 \\
\hline
\end{tabular}

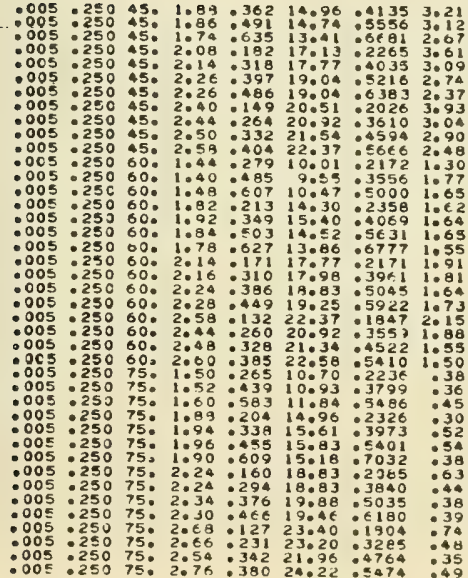

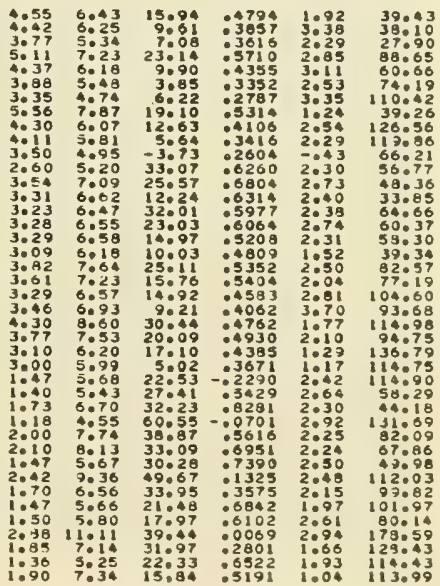




\begin{tabular}{|c|c|c|c|c|c|c|c|c|c|c|c|c|c|}
\hline & & G & 1 & H & L & & & & & PHI & . & $v$ & DV \\
\hline . & $\begin{array}{l}-2 \\
: 2 \\
: 2\end{array}$ & 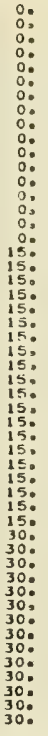 & 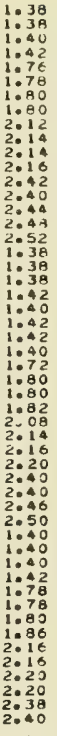 & 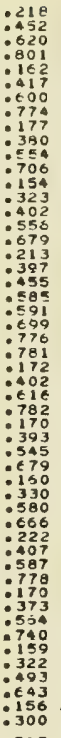 & 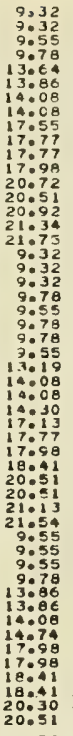 & 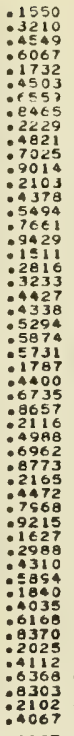 & 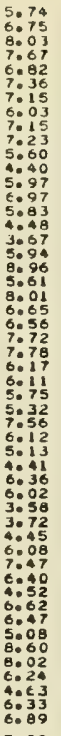 & 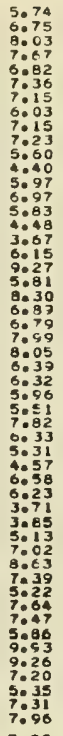 & $\begin{array}{l}5 \\
6 \\
8 \\
7 \\
6\end{array}$ & 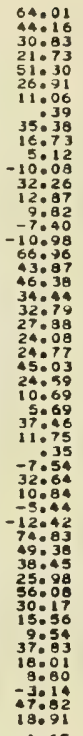 & 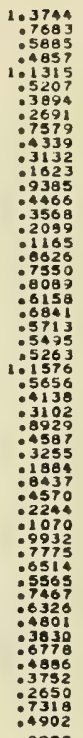 & $\begin{array}{r}4 \\
2 \\
1 \\
1 \\
7 \\
7 \\
2 \\
1 \\
1\end{array}$ & $\begin{array}{r}9 \\
181 \\
19 \\
20 \\
-5\end{array}$ \\
\hline io & $\begin{array}{l}250 \\
500 \\
50 \\
50 \\
50 \\
50\end{array}$ & 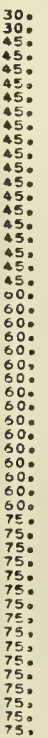 & 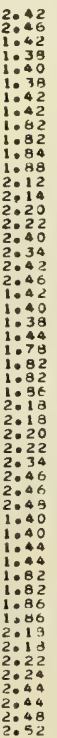 & 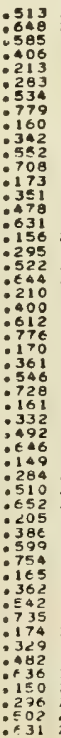 & $\begin{array}{l}9 \\
9 \\
9 \\
9 \\
14 \\
14\end{array}$ & 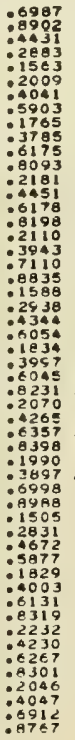 & $\begin{array}{l}40 \\
30 \\
20 \\
20 \\
30 \\
30 \\
40 \\
40 \\
30 \\
30 \\
30 \\
30 \\
30 \\
20 \\
10 \\
10 \\
10 \\
10\end{array}$ & : & & $\begin{array}{l}7 \\
3 \\
5 \\
1 \\
1 \\
7 \\
5 \\
3 \\
6 \\
7\end{array}$ & : $:$ & $\begin{array}{l}5 \\
2 \\
2 \\
2 \\
2\end{array}$ & 2 \\
\hline
\end{tabular}




\begin{tabular}{|c|c|c|c|c|c|c|c|c|c|c|c|c|c|}
\hline & $51 \mathrm{~m}$ & ANG & $\begin{array}{l}\top \\
\end{array}$ & $H$ & L & & ELV & 4 & CLVU & HI & K & IV & DV \\
\hline $\begin{array}{l}016 \\
016 \\
\text { Jit } \\
016 \\
016 \\
016 \\
016 \\
016 \\
016 \\
016 \\
016 \\
016 \\
016 \\
016 \\
016 \\
016 \\
016 \\
016 \\
016 \\
016 \\
016\end{array}$ & $\begin{array}{l}250 \\
250 \\
250 \\
250 \\
250 \\
250 \\
250 \\
250\end{array}$ & 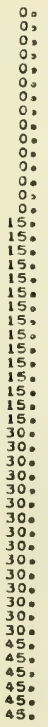 & $\begin{array}{l}1.34 \\
1.32 \\
1.40 \\
1: 74 \\
1.86 \\
1.82 \\
1.80 \\
2.02 \\
2.04 \\
2.020 \\
2.28 \\
2.26 \\
2.30 \\
2.34 \\
2.56 \\
1.32 \\
1.38 \\
1.52 \\
1: 74 \\
1.50 \\
1.92 \\
1.82 \\
2.04 \\
2.12 \\
2.24 \\
2.28 \\
2.32 \\
2.38\end{array}$ & 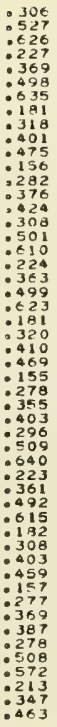 & 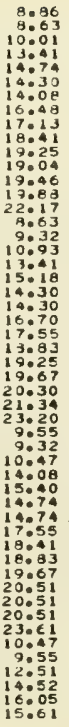 & 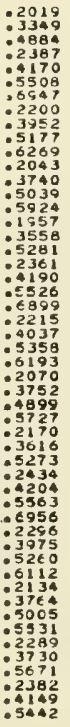 & $\begin{array}{l}8: \\
6: \\
9: \\
9: \\
7: \\
8: \\
9: \\
7: \\
8: \\
9:\end{array}$ & $\begin{array}{l}4.76 \\
7: 13 \\
8.54 \\
6: 04 \\
9: 08 \\
9: 25 \\
7.05 \\
8.08 \\
9.55 \\
7093 \\
7022 \\
8.01\end{array}$ & $\begin{array}{l}7 \\
8 \\
5 \\
9 \\
9 \\
7 \\
8\end{array}$ & 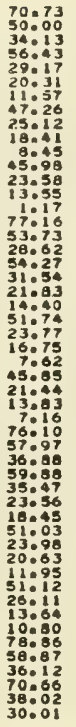 & $\begin{array}{l}352 \\
617 \\
959 \\
122 \\
964 \\
658 \\
615 \\
678 \\
703 \\
909 \\
021 \\
371 \\
788 \\
995 \\
571 \\
418 \\
389 \\
672 \\
693 \\
395 \\
007 \\
498 \\
612 \\
1912 \\
394 \\
664 \\
375 \\
936 \\
971\end{array}$ & $\begin{array}{r}3.42 \\
5.51 \\
3.28 \\
2.99 \\
3.02 \\
3.06 \\
-1.08 \\
20.54 \\
-20143 \\
4.961 \\
1.59 \\
4.83 \\
3.72 \\
6.84 \\
2.54 \\
-8.50 \\
6.19 \\
2.28 \\
3.28 \\
2.27 \\
2.31 \\
3.47 \\
-1.33 \\
1.17\end{array}$ & $\begin{array}{r}55 . \\
16 . \\
108 \\
10\end{array}$ \\
\hline
\end{tabular}

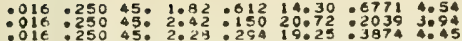

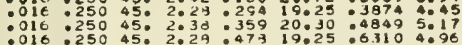

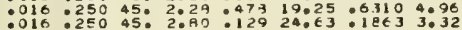
$016 \quad 250$ 45: $2.80:-12924.63 \quad 118 \epsilon 3 \quad 3.32$

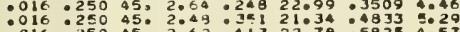

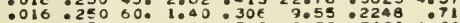

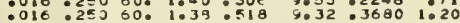
:016 050 50, 1.56 60911.380551011 .61 $: 016: 25060.1086: 221$ 14:74:02499 1.09 $\because 016$. 250 50. 1.32036515 .40 . 0254 1.\%

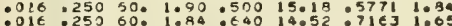
$: 016: 25060.2012: 161 \quad 17: 55: 22791: 43$ $.016 .25060 .2030 \cdot 398.17 .46 \cdot 52782.05$

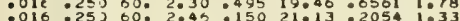
$0160^{250} 000$

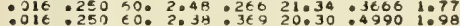
$: 01 \epsilon$ :250 60: 2:66:400 23:20:5680 1.82

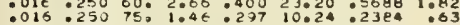

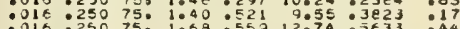

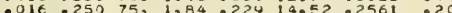
. Olt . $25075,1.94,360$ 15.61.4238:4 :016.250 75: 1:92:501 15.40:5839: . 016 . $25075: 1.86$ : 18 14.74: $1985: 5$ : 16.250 75. 2.22. I76 13.62 - 22A7: 6 . $016: 25075,2.30: 37020.09: 4980: 0$ - Jie. 250 75. 2.34. 4521 v. 48.0045 .61

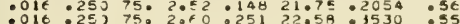

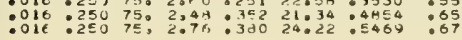

\begin{tabular}{|c|c|c|c|}
\hline 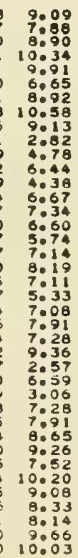 & 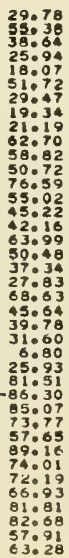 & 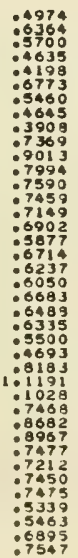 & 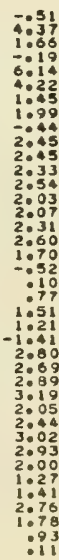 \\
\hline
\end{tabular}




\begin{tabular}{|c|c|c|c|c|c|c|c|c|c|c|c|c|c|}
\hline & A & i & 1 & $H$ & L & $M A X$ & LV & $V A$ & $v u$ & $1+1$ & K & $M V$ & DV \\
\hline $\begin{array}{l}021 \\
0221 \\
321 \\
021 \\
021 \\
021 \\
021 \\
021 \\
021 \\
021 \\
021 \\
021 \\
021 \\
021 \\
021\end{array}$ & $\begin{array}{l}250 \\
250 \\
250 \\
250 \\
250 \\
250 \\
250 \\
250 \\
250 \\
250 \\
250 \\
250 \\
250 \\
250 \\
250 \\
250 \\
250 \\
250 \\
250 \\
250 \\
250 \\
250 \\
250\end{array}$ & 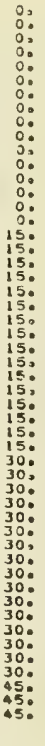 & $\begin{array}{l}1038 \\
1034 \\
1042 \\
1042 \\
1075 \\
1082 \\
1080 \\
1082 \\
2010 \\
2010 \\
2003 \\
2016 \\
2034 \\
2032 \\
2038 \\
2042 \\
1038 \\
1038 \\
1042 \\
1042 \\
1076 \\
1079 \\
1082 \\
1084 \\
2010 \\
3014 \\
2016 \\
2027 \\
2042 \\
2042 \\
2344 \\
2045 \\
1038 \\
1036 \\
1040 \\
1042 \\
1072 \\
1074 \\
1080 \\
1080 \\
2014 \\
2010 \\
2016 \\
2018 \\
2039 \\
2044 \\
2048 \\
2046\end{array}$ & 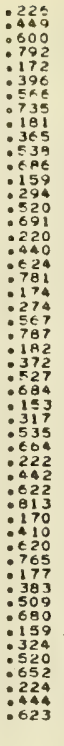 & 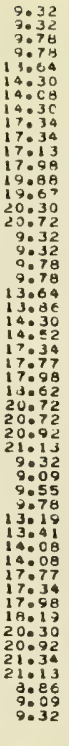 & 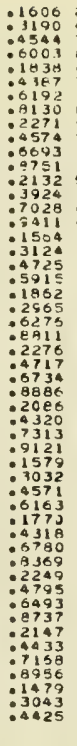 & 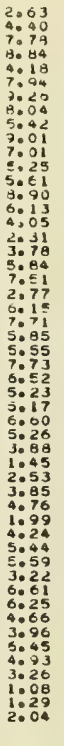 & $?$ & 7 & 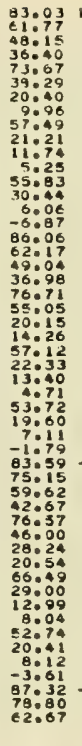 & 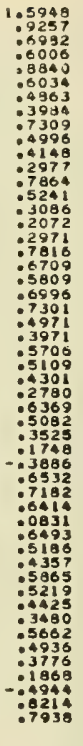 & $\begin{array}{l}1 \\
2 \\
-8 \\
2 \\
3 \\
3 \\
1 \\
10\end{array}$ & $\begin{array}{l}4 \\
1 \\
1 \\
2 \\
3 \\
3 \\
3 \\
6 \\
9 \\
7 \\
4\end{array}$ \\
\hline $\begin{array}{l}021 \\
021 \\
021 \\
021 \\
021 \\
021 \\
021 \\
021 \\
021 \\
021 \\
021 \\
021 \\
021 \\
021 \\
021 \\
021 \\
021 \\
021 \\
021 \\
021\end{array}$ & $\begin{array}{l}\cdot 25 \\
: 25 \\
: 25 \\
: 25 \\
: 25 \\
: 25 \\
: 25 \\
: 25 \\
: 25 \\
: 25 \\
: 25 \\
: 25 \\
: 25 \\
: 25 \\
: 25 \\
: 25 \\
: 25 \\
: 25\end{array}$ & 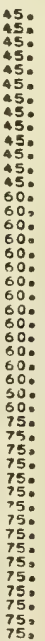 & $\begin{array}{l}2: \\
2: \\
1: \\
1: \\
1: \\
1: \\
1:\end{array}$ & 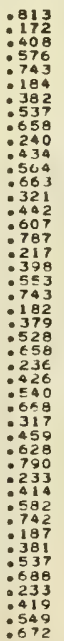 & $\begin{array}{l}20 \\
20 \\
3 \\
50 \\
9 \\
9 \\
13 \\
13 \\
14 \\
14 \\
17 \\
17 \\
18 \\
18\end{array}$ & 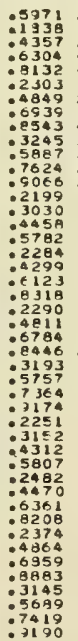 & $\begin{array}{l}2 \\
4 \\
4 \\
1 \\
2 \\
3 \\
4\end{array}$ & 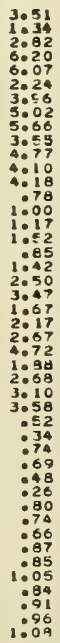 & & $\begin{array}{l}9 \\
7 \\
5 \\
3 \\
1 \\
6 \\
3 \\
2 \\
1 \\
5 \\
1 \\
1 \\
1 \\
8 \\
8 \\
7 \\
6 \\
8 \\
7 \\
5 \\
3 \\
8 \\
5 \\
4 \\
2 \\
6 \\
4 \\
3 \\
2 \\
4 \\
6 \\
7 \\
8 \\
4\end{array}$ & : & $\begin{array}{r}171 \\
687 \\
198 \\
988 \\
114 \\
179\end{array}$ & $\begin{array}{r}11 \\
5 \\
57 \\
23 \\
38 \\
27 \\
35 \\
34 \\
70 \\
71 \\
44 \\
-11\end{array}$ \\
\hline
\end{tabular}




\begin{tabular}{|c|c|c|c|c|c|c|c|c|c|c|c|c|c|}
\hline & DIA & ANS & $T$ & $H$ & L & $J M A x$ & LV & LVA & $-v \cdot s$ & PHI & $k$ & CMV & COV \\
\hline $\begin{array}{l}042 \\
0442 \\
0442 \\
0442 \\
0442 \\
042 \\
0442 \\
0442 \\
0442 \\
0442 \\
042 \\
042 \\
042 \\
042 \\
0442 \\
042 \\
042 \\
042 \\
042\end{array}$ & 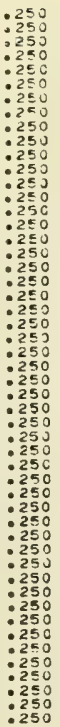 & 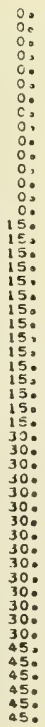 & 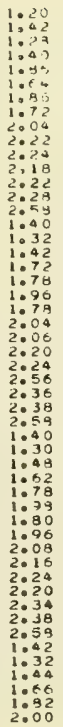 & 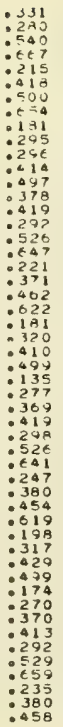 & 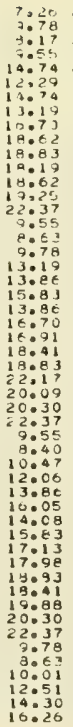 & 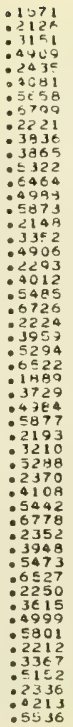 & 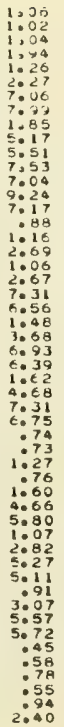 & 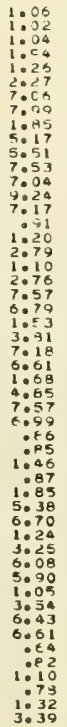 & $\begin{array}{l}3.75 \\
7: 02 \\
0.82 \\
1: 21 \\
4: 09 \\
7: 43 \\
7: 63 \\
1: 91 \\
1: 16 \\
1: 56 \\
1.11\end{array}$ & $\begin{array}{l}87: 05 \\
66: 17 \\
85: 54 \\
69: 92 \\
82: 94 \\
69: 20 \\
41: 89 \\
34: 93 \\
77.69 \\
55: 59 \\
57: 38 \\
37: 20 \\
26: 21 \\
32: 69 \\
17.37 \\
85.98 \\
83.22 \\
64: 31 \\
83.24\end{array}$ & 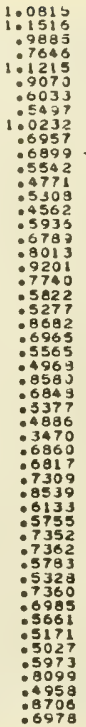 & $\begin{array}{r}2.58 \\
2.17 \\
3.13 \\
3.51 \\
1.85 \\
1: 69 \\
1.57 \\
4.58 \\
1.25 \\
3.11 \\
-1.44 \\
3.02 \\
6.93 \\
8.59 \\
3.83 \\
2.96 \\
3.63 \\
-.56 \\
1.99 \\
2.63 \\
4.00 \\
8.04\end{array}$ & $\begin{array}{r}25.70 \\
22.70 \\
36.94 \\
45.59 \\
11.70 \\
17.43 \\
-.01 \\
13.03 \\
32.21 \\
65014 \\
-25.01 \\
93.37 \\
173.01 \\
100.30 \\
179.93 \\
28.57 \\
36.64 \\
11.08 \\
26.96 \\
62.55 \\
79.42 \\
67.57 \\
32.65 \\
114.80 \\
118.51 \\
113.89 \\
51.31 \\
-51.25\end{array}$ \\
\hline
\end{tabular}

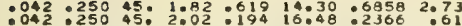
$\because 042: 25 \mathrm{C}$ 45: $2,10: 31917.34: 3991 \quad 1033$ $: 042: 25045: 2.24: 406 \quad 18.83: 5304 \quad 2.57$ $042: 250 \quad 45: 2.30: 1 \in 3 \quad 19.46: 2160: 76$ $\because 042: 250$ 45: $2.40: 37320.51: 56562.60$ .042 . $042 \cdot 250 \quad 60.1 .38 \cdot 297 \quad 9.32 \cdot 2115 \cdot 19$ $: 042$ :250 50, $1: 40: E 23 \quad 10.24: 5 \mathrm{C} D 5: 40$

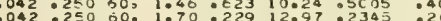
$042: 2 E 500: 1096: 36714.74: 9155: 41$ $: 042$ : 250 3c: $1: 98$ : 443 le:05 0554 : 75 .042 .250 60: $1.78: 637$ i3.86:0893:80 $\because 042 \cdot 25060.204: 186 \quad 16.70: 2281 \quad: 41$ $042: 25050: 2.20: 40418.41: 5225: 94$

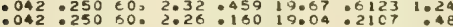
$\because 042: 25060: 2.32: 281 \quad 19.67 \cdot 3749: 55$ $\because 042: 25060: 2.38: 366 \quad 20: 30: 4542 \quad: 94$ $\because 042$ :250 60: $2.64: 397 \quad 23.40: 5353 \quad 1.31$ $.042 \cdot 25075 \cdot 1.38 \cdot 520 \quad 9 \cdot 32 \cdot 1700 \cdot 25$ $: 042: 2 \leq 0750$ : : $86: 359 \quad 14: 74: 0050: 28$

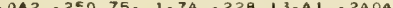
$: 042: 25 c 7502004: 442 \quad 16.70: 5424: 35$ $040.2507502004: 442 \quad 16.700: 0424: 35$ $: 042: 25075: 2.02: 17216: 48: 2335: 33$ $: 042: 25075: 2.16: 30818,19: 3957: 23$

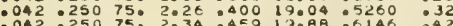
$.042 .250 \quad 75.2 .34 .45917 .88 .0146 .42$

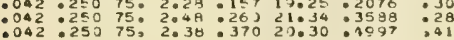
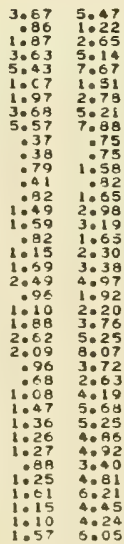

$55.32 \quad .6274$

3:71

$54: 86$

$2.65 \quad 71.65 \quad 07612 \quad 3.31 \quad 70.75$

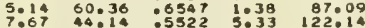
$\begin{array}{lllll}1.91 & 83.52 & : 8285 & 2.35 & 49.38\end{array}$

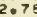
5. 21 $7: 88$ 87.908 58.55 40.56002 103.39 :7457 1.58 96.65 1.1443 $1.05060 .25-1065$ 2.98 77072 :9357 (2.105 $2: 30$ 93524 3. 3876.38 $4: 97 \quad 61: 98$ 2.207
30 5.25 64:91 $8.07 \quad-4.4$ $\begin{array}{ll}3.72 & -28: 18 \\ 2.63 & 109.20\end{array}$ $4: 191_{104}^{1008}$ 5: $64-37: 15$ 5.2596 .57 40.85 84.34 3:92 $-590: 42$

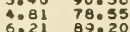
$6.21 \quad 89.20: 7461$ 4.45

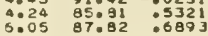

49.38
75.36

24.47

$10 \% .22$

70.59

48.82

69.59

52.19

98.88

97.27

88.1

$95 \% 1$

72.48

127.81

136.8

$57: 80$

79055

58.08

59.08

128.39

66.91

132.64

$74: 71$ 


\begin{tabular}{|c|c|c|c|c|c|c|c|c|c|c|c|c|c|}
\hline & $1 \mathrm{~A}$ & Niv & 1 & H & L & $\sin A x$ & LV & CLVA & $u$ & PHI & K & iv & LV \\
\hline 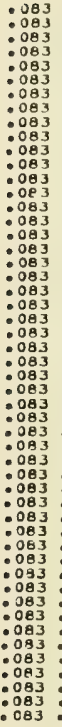 & 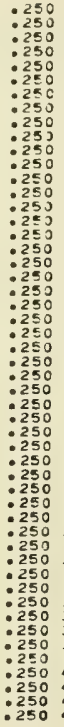 & 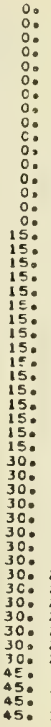 & 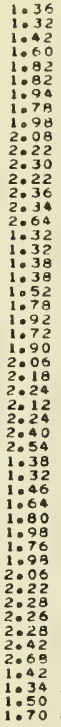 & 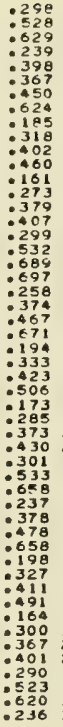 & $\begin{array}{r}9.09 \\
8.63 \\
9.78 \\
11.84 \\
14.30 \\
14.30 \\
15.61 \\
13.86 \\
16.05 \\
17.13 \\
18.62 \\
19.46 \\
19.62 \\
20.09 \\
19.88 \\
22.99 \\
8.63 \\
8.63 \\
9.32 \\
9.32 \\
10.93 \\
13.86 \\
15.40 \\
13.11 \\
15018 \\
16.91 \\
18.19 \\
19.83 \\
17.55 \\
19.83 \\
20.51 \\
21.96 \\
9.32 \\
8.63 \\
10.24 \\
12.29 \\
14.08 \\
16.05 \\
13.64 \\
16.05\end{array}$ & 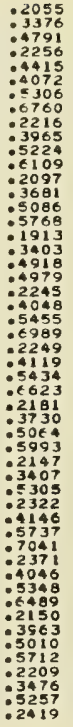 & 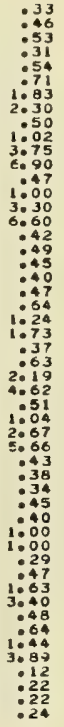 & $\begin{array}{l}: 3 \\
: 5 \\
: 3 \\
: 5 \\
1: 8 \\
2: 3 \\
1: 5 \\
3: 5 \\
6: 9 \\
1: 5\end{array}$ & $:$ & $\begin{array}{l}3 \\
4 \\
4 \\
6 \\
4 \\
0 \\
1 \\
3 \\
0 \\
9 \\
9 \\
1 \\
4 \\
3 \\
1 \\
4\end{array}$ & $:$ & $\begin{array}{r}2 \\
1 \\
1 \\
1 \\
1 \\
-1 \\
1 \\
1 \\
1 \\
1 \\
2 \\
5 \\
2 \\
2 \\
1 \\
2 \\
2\end{array}$ & $\begin{array}{r}-1 \\
-1 \\
-1 \\
-1 \\
2 \\
2 \\
-2 \\
1 \\
1 \\
-1 \\
-1 \\
-1 \\
1 \\
-2 \\
1 \\
3\end{array}$ \\
\hline
\end{tabular}

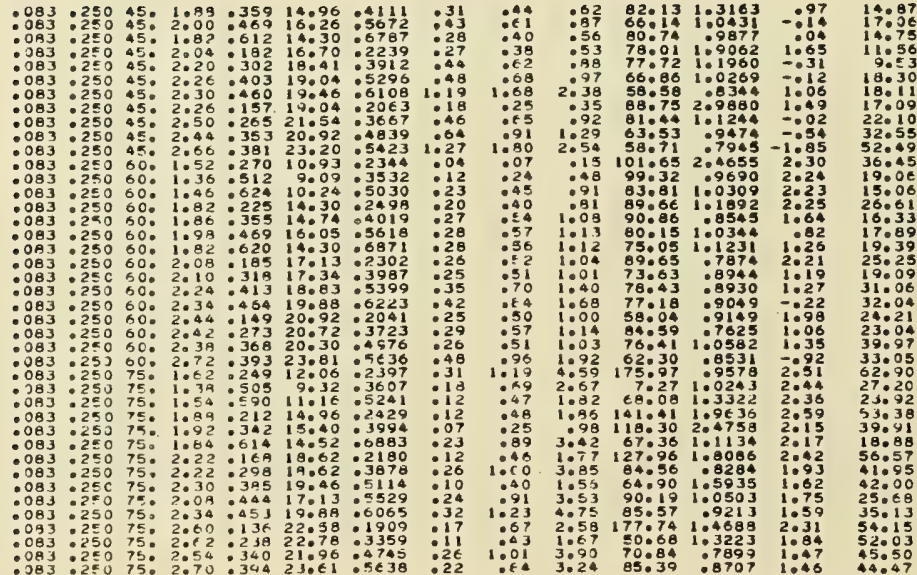




\begin{tabular}{|c|c|c|c|c|c|c|c|c|c|c|c|c|c|}
\hline$=R$ & Ia & NG & $T$ & $H$ & L & $A x$ & LV & A & 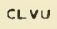 & HI & $K$ & IV & $0 \mathrm{~V}$ \\
\hline $\begin{array}{l}167 \\
167 \\
167 \\
167 \\
167 \\
167 \\
167 \\
167 \\
167 \\
167 \\
167 \\
167 \\
167 \\
167 \\
167 \\
167 \\
167 \\
167 \\
167 \\
167 \\
167 \\
167 \\
167 \\
167 \\
167 \\
167 \\
167 \\
167 \\
167 \\
167 \\
167 \\
167 \\
167 \\
167 \\
167 \\
167 \\
167 \\
167 \\
167 \\
167 \\
167 \\
167 \\
167 \\
167 \\
167 \\
167 \\
167 \\
167 \\
167\end{array}$ & 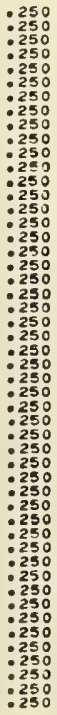 & 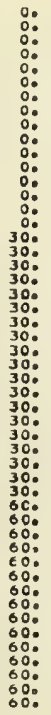 & $\begin{array}{l}1.60 \\
1.42 \\
1.46 \\
1.48 \\
1.90 \\
1.95 \\
2.04 \\
1.78 \\
2.26 \\
2.24 \\
2.42 \\
2.28 \\
2.74 \\
2.66 \\
2.22 \\
2.64 \\
1.56 \\
1.38 \\
1.50 \\
1.54 \\
1.88 \\
1.96 \\
2.04 \\
2.02 \\
2.02 \\
1.86 \\
2.26 \\
2.16 \\
2.34 \\
2.26 \\
2.32 \\
2.58 \\
2.56 \\
2.40 \\
2.66 \\
1.50\end{array}$ & 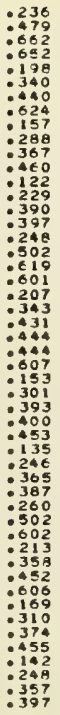 & 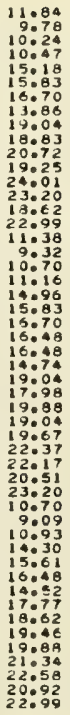 & 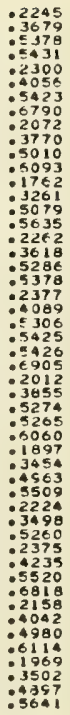 & 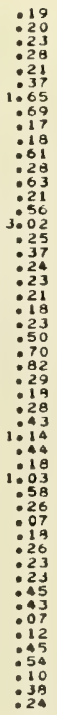 & 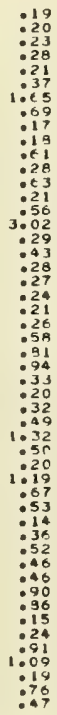 & 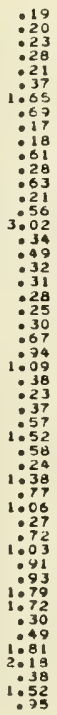 & 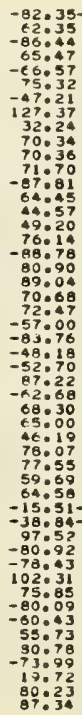 & 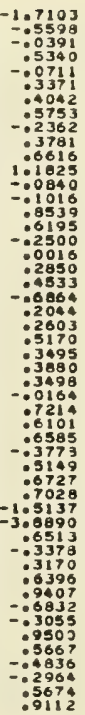 & 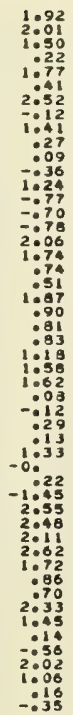 & $\begin{array}{l}85 \\
71 \\
91 \\
59 \\
28 \\
35 \\
52 \\
29 \\
13 \\
23 \\
63 \\
15 \\
89 \\
39 \\
25 \\
39 \\
15 \\
11 \\
89 \\
07 \\
97\end{array}$ \\
\hline
\end{tabular}




\begin{tabular}{|c|c|c|c|c|c|c|c|c|c|c|c|c|c|}
\hline & $1 \mathrm{~A}$ & ANG & $T$ & H & L & UMAX & CLV & CLVA & LLVU & PHI & k & SAV & COV \\
\hline 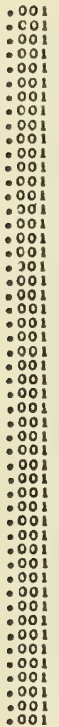 & 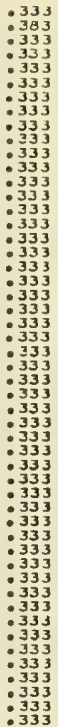 & 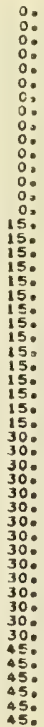 & 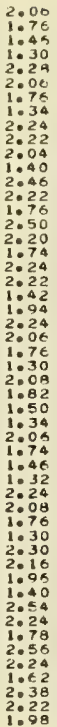 & 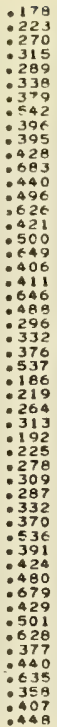 & $\begin{array}{l}16.91 \\
13.68 \\
10.24 \\
8.40 \\
19.25 \\
16.91 \\
13.64 \\
8.86 \\
18.83 \\
18.62 \\
16.70 \\
7.55 \\
21.13 \\
18.62 \\
13.64 \\
21.54 \\
18.41 \\
13.41 \\
18.93 \\
18.62 \\
9.78 \\
15.61 \\
18.83 \\
16.91 \\
13.64 \\
1.40 \\
17.13 \\
14.30 \\
10.70 \\
8.86 \\
16.91 \\
13.41 \\
10.24 \\
3.63 \\
18.83 \\
17.13 \\
13.64 \\
8.40 \\
10.46 \\
17.98 \\
15.83 \\
9.55 \\
21.96 \\
18.83 \\
13.86 \\
22.17 \\
18.83 \\
12.08 \\
20.30 \\
18.62 \\
16.05\end{array}$ & 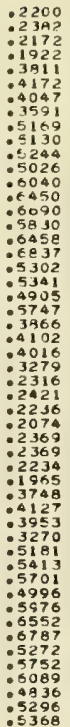 & 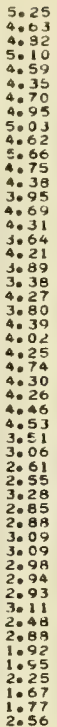 & 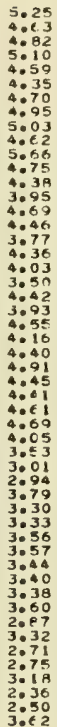 & 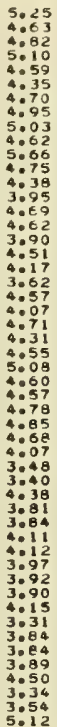 & 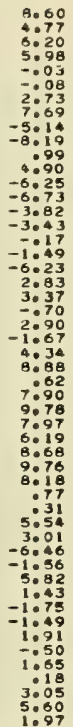 & $\begin{array}{r}.0780 \\
1223 \\
11660 \\
12018 \\
00432 \\
0502 \\
00999 \\
01525 \\
00332 \\
0193 \\
01593 \\
01040 \\
1040 \\
0023 \\
00013\end{array}$ & $\begin{array}{r}4.24 \\
3.11 \\
2.95 \\
2.76 \\
5.19 \\
3.50 \\
3.43 \\
3.52 \\
2.52 \\
3.64 \\
2.99 \\
2.57 \\
.66 \\
3.28 \\
3.68 \\
1.55 \\
1.19 \\
1.55 \\
1.97 \\
1.79 \\
2.93 \\
1.36 \\
1.48\end{array}$ & $\begin{array}{r}290.19 \\
150.50 \\
109.64 \\
96: 38 \\
162.18 \\
134.39 \\
103.24 \\
74.83 \\
140.14 \\
147.76 \\
138.46 \\
62.54\end{array}$ \\
\hline
\end{tabular}

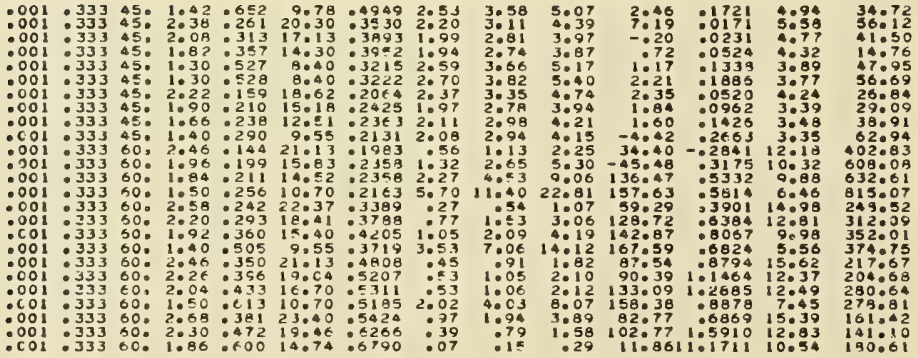




\begin{tabular}{|c|c|c|c|c|c|c|c|c|c|c|c|c|c|}
\hline & 14 & $\sqrt{5}$ & T & H & $L$ & $\operatorname{MAx}$ & $v$ & $\checkmark A$ & cero & H t & K & MV & DV \\
\hline 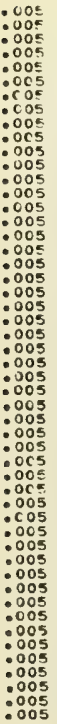 & 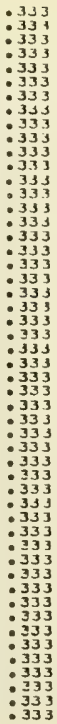 & $\begin{array}{l}0 \\
0 \\
0 \\
0 \\
0 \\
0 \\
0 \\
0 \\
0 \\
0 \\
0 \\
0 \\
0 \\
0 \\
0 \\
0 \\
13 \\
15 \\
15 \\
15 \\
15 \\
15 \\
15 \\
150 \\
150 \\
15 \\
150 \\
15 \\
150 \\
150 \\
150 \\
30 \\
30 \\
30 \\
30 \\
30 \\
30 \\
30 \\
30 \\
30 \\
30 \\
30 \\
30 \\
30 \\
30 \\
30 \\
450 \\
15\end{array}$ & 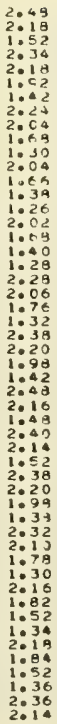 & 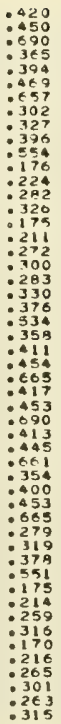 & 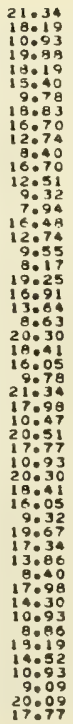 & 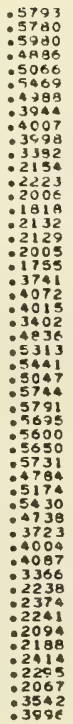 & 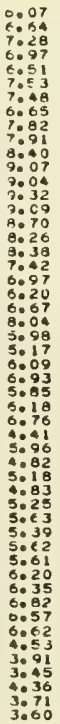 & $\begin{array}{l}0.07 \\
6.04 \\
7028 \\
6.07 \\
6.31 \\
7.53 \\
7.42 \\
0.65\end{array}$ & ?. & $\begin{array}{r}31 \\
38 \\
43 \\
9 \\
13 \\
19 \\
26 \\
12 \\
11 \\
18 \\
1 \\
18 \\
12 \\
16 \\
15 \\
13\end{array}$ & 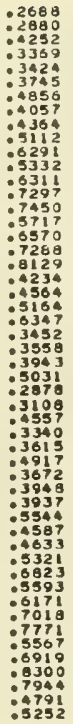 & & 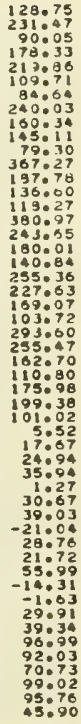 \\
\hline
\end{tabular}
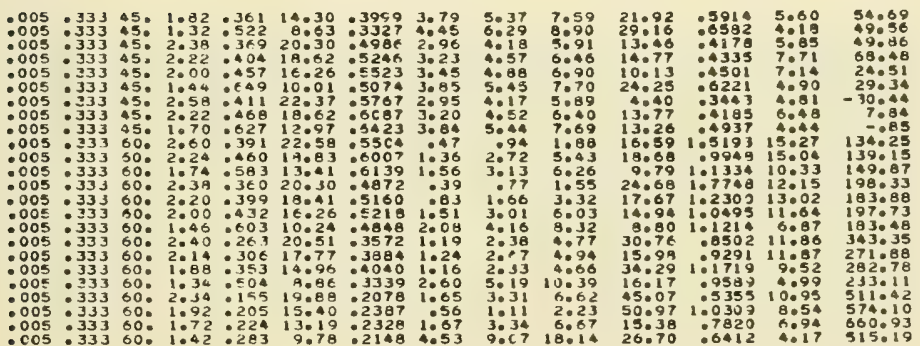


\begin{tabular}{|c|c|c|c|c|c|c|c|c|c|c|c|c|c|}
\hline$E F$ & CIA & $N=$ & $T$ & $H$ & L & UMAX & CLV & CLVA & CLVU & $2+1$ & $k$ & CMV & $=0 \mathrm{~V}$ \\
\hline 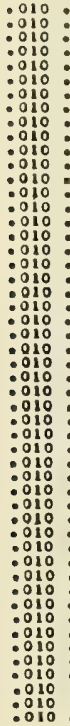 & 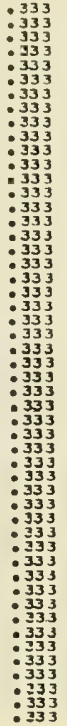 & 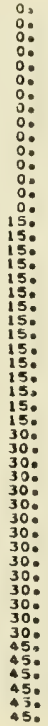 & 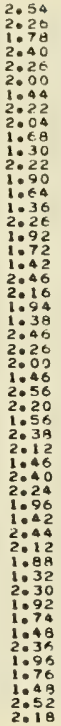 & 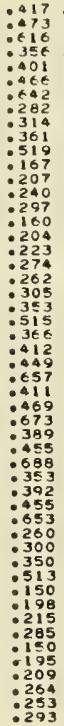 & $\begin{array}{l}21.96 \\
19.04 \\
13.86 \\
20.51 \\
19.04 \\
16.26 \\
10.01 \\
18.62 \\
16.70 \\
12074 \\
8.40 \\
18.62 \\
15.18 \\
12.29 \\
9.09 \\
19.04 \\
15.40 \\
13.19 \\
9.78 \\
21.13 \\
17.88 \\
19.81 \\
9.32 \\
21.13 \\
19.04 \\
16.26 \\
10.24 \\
22.17 \\
18.41 \\
11.38 \\
20.30 \\
17.55 \\
10.24 \\
20.51 \\
18.83 \\
15.83 \\
9.78 \\
20.92 \\
17.55 \\
14.96 \\
8.63 \\
19.46 \\
15.40 \\
13.41 \\
10.47 \\
20.09 \\
15.83 \\
13.64 \\
10.47 \\
21.75 \\
18.19\end{array}$ & 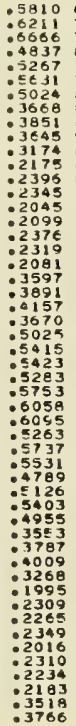 & 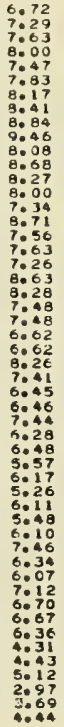 & 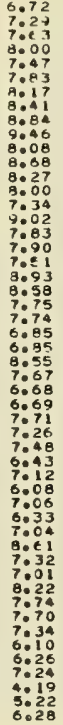 & 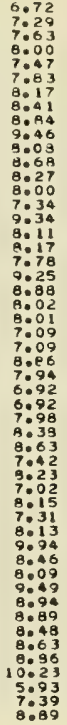 & 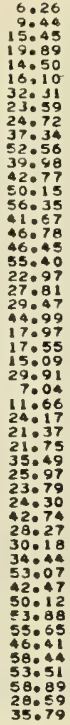 & $\begin{array}{l}: 4 \\
: 4 \\
: 4 \\
: 4 \\
: 6 \\
: 5 \\
: 5 \\
: 6 \\
: 7 \\
: 7 \\
: 8 \\
: 8 \\
: 7 \\
: 8 \\
: 8 \\
: 5 \\
: 5 \\
: 6 \\
: 4 \\
: 4 \\
: 5 \\
: 6 \\
: 4 \\
: 5 \\
: 4 \\
: 4 \\
: 5 \\
: 5 \\
: 5 \\
: 6 \\
: 5\end{array}$ & 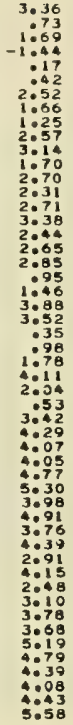 & 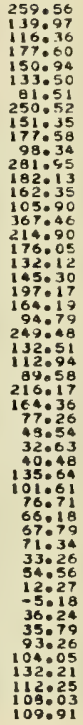 \\
\hline
\end{tabular}

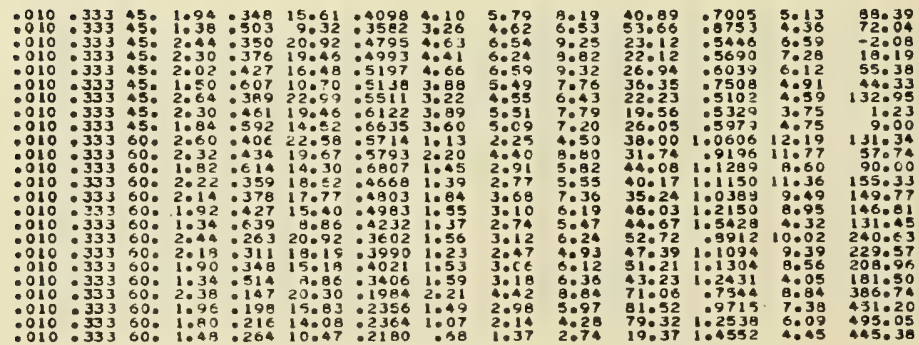




\begin{tabular}{|c|c|c|c|c|c|c|c|c|c|c|c|c|c|}
\hline & $1 \mathrm{~A}$ & NS & $T$ & $H$ & $\mathbf{L}$ & $\max$ & CLV & LVA & LVJ & HI & K & MV & :DV \\
\hline $\begin{array}{l}01 \\
01 \\
01 \\
01 \\
01 \\
01 \\
01 \\
01 \\
01 \\
01\end{array}$ & $\begin{array}{l}: 333 \\
: 333 \\
: 333 \\
: 333 \\
: 333 \\
: 333 \\
: 333 \\
: 333 \\
: 333 \\
: 333 \\
: 333 \\
: 333 \\
: 333 \\
: 333 \\
: 333 \\
: 333 \\
: 333 \\
: 333 \\
: 333 \\
: 333 \\
: 333 \\
: 333 \\
: 333 \\
: 333 \\
: 333 \\
: 333 \\
: 333 \\
: 333 \\
: 333 \\
: 333 \\
: 333 \\
: 333 \\
: 333 \\
: 333 \\
: 333 \\
: 333 \\
: 333 \\
: 333 \\
: 333 \\
: 333 \\
: 333 \\
: 333 \\
: 333 \\
: 333 \\
: 333 \\
: 333 \\
: 333 \\
: 333 \\
: 333 \\
: 333 \\
: 333 \\
: 333 \\
: 333 \\
: 333 \\
: 333 \\
: 333 \\
: 333 \\
33\end{array}$ & 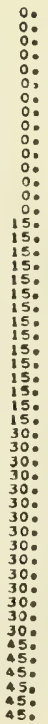 & $\begin{array}{l}2: 40 \\
2: 18 \\
1: 56 \\
2.32 \\
2.16 \\
1: 90 \\
1: 36 \\
2: 22 \\
1: 99 \\
1: 52 \\
1: 32 \\
1.76\end{array}$ & 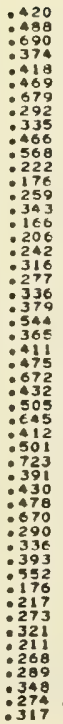 & 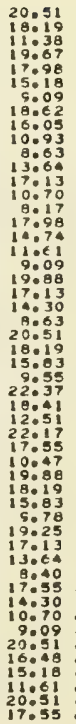 & 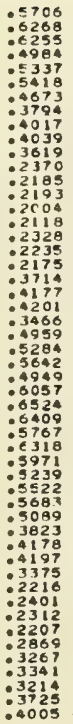 & 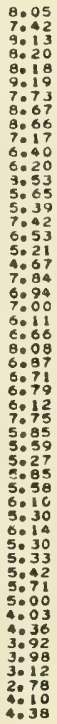 & $\begin{array}{l}8: 0 \\
7: 5 \\
8: 1 \\
8: 2 \\
8: 1 \\
9: 1 \\
7: 7 \\
8: 0 \\
8: 0 \\
7: 1 \\
6.4\end{array}$ & $\begin{array}{l}8: 1 \\
8: 2 \\
8: 1 \\
9: 1 \\
7: \\
8: \\
8: \\
7:\end{array}$ & 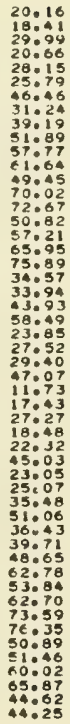 & 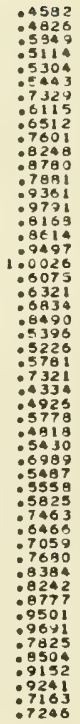 & $\begin{array}{l}.23 \\
111 \\
120 \\
.68 \\
.71 \\
.65 \\
.93 \\
.99\end{array}$ & $\begin{array}{r}72.31 \\
253.00 \\
85.63 \\
109.05 \\
70.50 \\
159.03 \\
109.45 \\
59.51 \\
53.60 \\
32.74 \\
146.90 \\
65.43 \\
56.71 \\
94.64 \\
53.15 \\
41.56 \\
57.63 \\
185.09 \\
148.25 \\
57.80 \\
42.25 \\
220.16 \\
60.94 \\
100.46 \\
54.87 \\
176.04 \\
139.83 \\
48.64 \\
110.39 \\
65.87 \\
55.10 \\
74.70 \\
105.07 \\
76.50 \\
55.74 \\
91.87 \\
15.22 \\
10.18 \\
34.68 \\
-32.57 \\
-11.36 \\
8.53 \\
16.93 \\
58.70 \\
61.29 \\
11.80 \\
36.44 \\
85.44 \\
104.84\end{array}$ \\
\hline
\end{tabular}

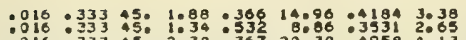

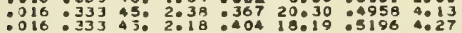

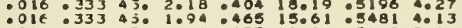

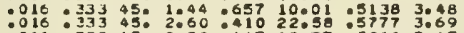
$: 016: 3334502.32: 44719.67 .99665047$ $: 016: 3334501078$ :640 $13: 86: 70143.65$ .016 : $3336002.54043721 .56: 8089$ 1.85

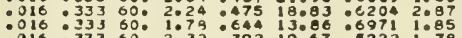
$: 316: 33360: 2.32: 392$ 19:67: $: 222 \quad 1: 78$

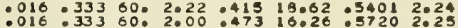

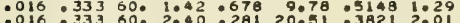

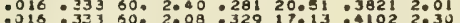
$0.518: 333$ 50: $2: 08: 329 \quad 17: 13: 4102 \quad 2: 30$ $\because 16: 33360: 1: 80037514: 08: 410331050$ $: 016: 33350: 2024: 168$ 18.83 :2197 $1: 95$

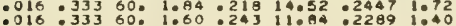
$: 016: 33960: 1036: 310 \quad 9009: 2132 \quad: 60$

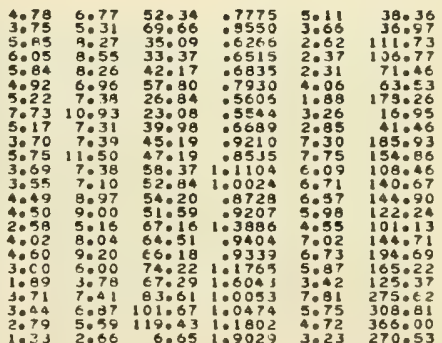




\begin{tabular}{|c|c|c|c|c|c|c|c|c|c|c|c|c|c|}
\hline EF & CIA & $A N G$ & $r$ & $M$ & L & UMAX & CLV & CLVA & CLVU & DHI & $k$ & $C M V$ & CDV \\
\hline $\begin{array}{l}.021 \\
021 \\
021 \\
021 \\
021 \\
021 \\
021 \\
021 \\
021 \\
021 \\
021 \\
021 \\
021 \\
021 \\
021 \\
021 \\
021 \\
021 \\
021 \\
021 \\
021 \\
021 \\
021 \\
021 \\
021 \\
021 \\
021 \\
021 \\
021 \\
021 \\
021 \\
021 \\
021 \\
021 \\
021 \\
021 \\
021 \\
021 \\
021 \\
021 \\
021 \\
021 \\
021 \\
021 \\
021 \\
021 \\
021 \\
021 \\
021 \\
021 \\
021 \\
021 \\
021 \\
021 \\
021 \\
021 \\
021 \\
021 \\
021 \\
021 \\
021 \\
021 \\
021 \\
021 \\
021 \\
021 \\
021\end{array}$ & 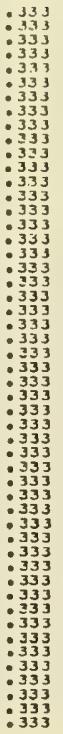 & 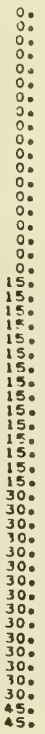 & 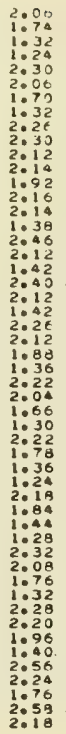 & 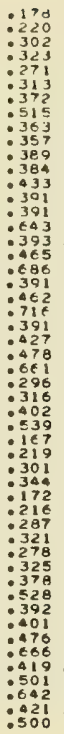 & 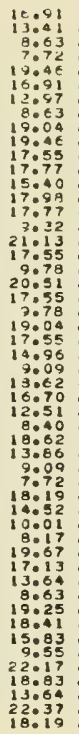 & 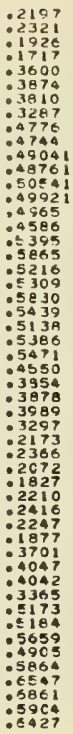 & 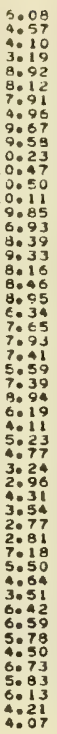 & 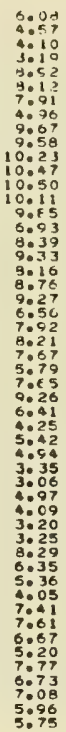 & 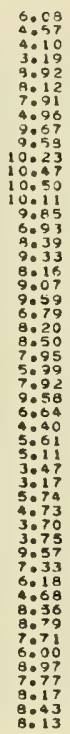 & 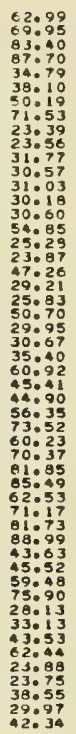 & 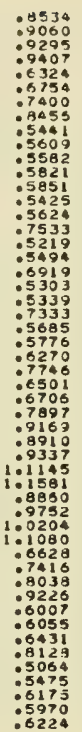 & 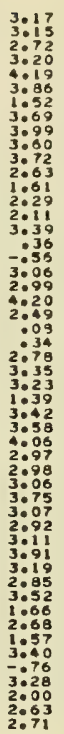 & $\begin{array}{r}141.96 \\
55.85 \\
59: 38 \\
83.85 \\
203.09 \\
125.05 \\
89.72 \\
61.80 \\
190.14 \\
255.73 \\
96.06 \\
103.11 \\
63.19 \\
69.60 \\
94.08 \\
54.07 \\
180.78 \\
81.56 \\
71.82 \\
134.54 \\
101.35 \\
48.55 \\
93.07 \\
67.69 \\
87.84 \\
51.42 \\
156.53 \\
62.99 \\
57.39 \\
42.44 \\
85.64 \\
62.81 \\
48.02 \\
55.23 \\
-39.33 \\
-4.07 \\
1.20 \\
31.97 \\
80.79 \\
19.71 \\
28.09 \\
21.61 \\
45.72 \\
50.36 \\
48.11 \\
32.62\end{array}$ \\
\hline
\end{tabular}

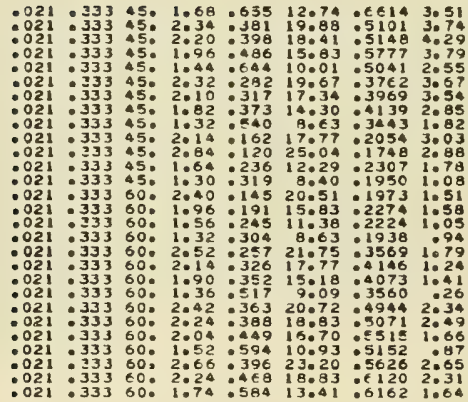

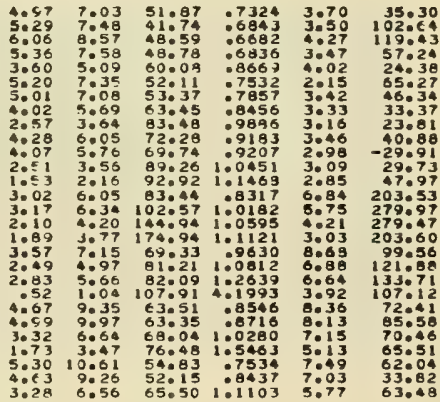




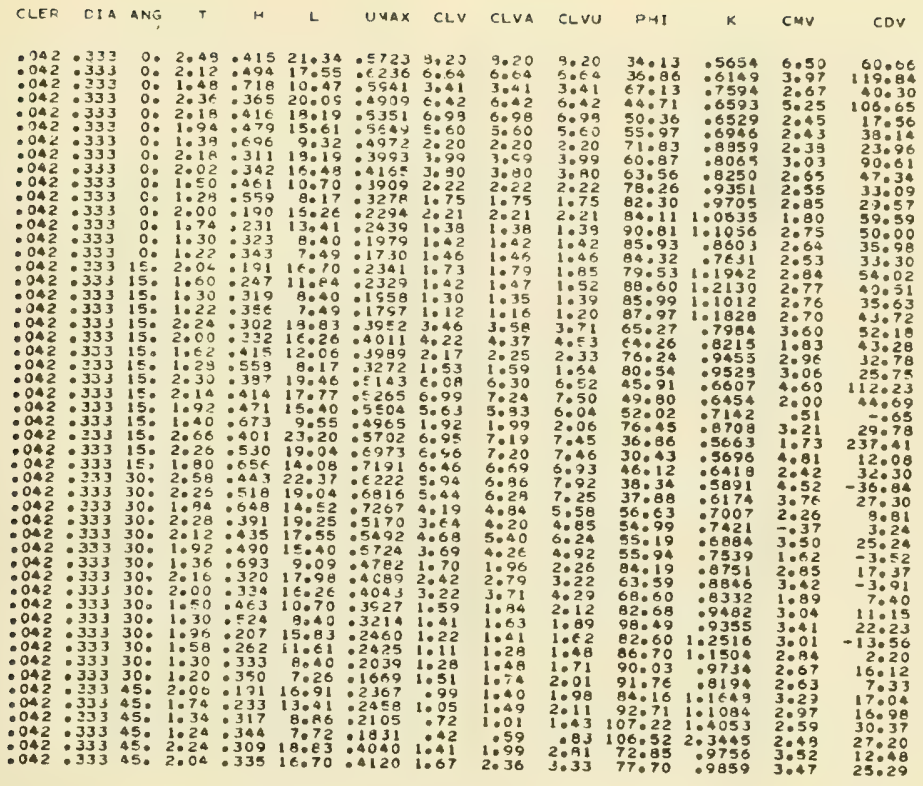

$: 042: 33345: 1: 72: 392 \quad 13: 19: 4083 \quad 1,19$

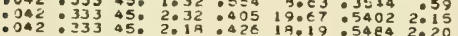
$042.33345,1.94 \cdot 494 \quad 15.61 .59212 .00$ $.0420 .3345 .1 .42 .090 \quad 3074.51781 .06$

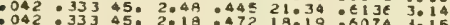
$: 042: 33345: 2: 18: 472 \quad 19: 199: 5074 \quad 1: 15$ $042: 33360,2.50: 00$ 2 $21.54: 55601.45$

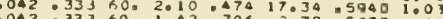
$.042: 333 \quad 60: 2034: 384 \quad 3778: 0373: 41$ $.042 .33360 .2 .19 .01618 .19: 5349$ 1. 30 $.042: 333$ 80: $1.99: 046 \quad 15005$ :5822 $: 05$ $.042: 333$ o0, 1.4e: $05310.24: 5261: 38$ $.042: 333$ 60: $2.34: 28 E$ 19:Ae: उeद3 :90 $042 \cdot 33330,2.10 \cdot 322 \quad 17.34: 0039: 97$ $.042: 33360$. 1. $022: 37414: 30: 4153$ :00 $.042 \cdot 33360 \cdot 1 \cdot 32 \cdot 545 \quad 8 \cdot E 3: 3497: 50$ $.042: 33300: 1.82: 21614.32: 23971.06$

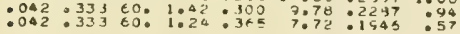

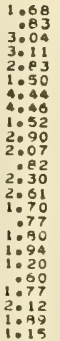

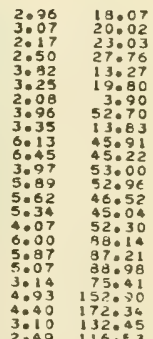


APPENDIX G

TABULATED HORIZONTAL FORCE DATA

FROM TWO-DIMENSIONAL EXPERIMENTS 


\begin{tabular}{|c|c|c|c|c|c|c|c|c|c|}
\hline$L \equiv R$ & OIA & NG & $T$ & H & L & UMAX & $\mathrm{CMH}$ & $\mathrm{COH}$ & $F A \vee G$ \\
\hline 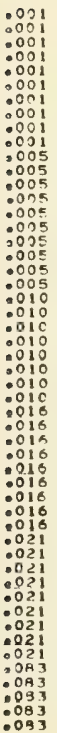 & 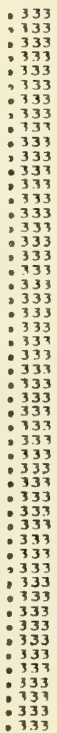 & 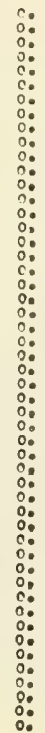 & 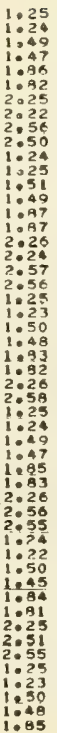 & 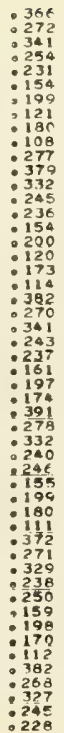 & 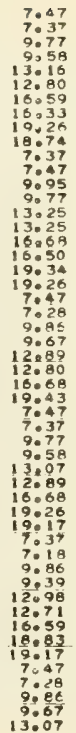 & $\begin{array}{l}\text { : } 3579 \\
2623 \\
: 4323 \\
: 3168 \\
: 3529 \\
: 2329 \\
: 3345 \\
: 2032 \\
: 3169 \\
: 1862 \\
: 2672 \\
: 3705 \\
: 4272 \\
: 3105 \\
: 3419 \\
: 2365 \\
: 3367 \\
: 2006 \\
: 3040 \\
: 1995 \\
: 3741 \\
: 2541 \\
: 4354 \\
: 3063 \\
: 3584 \\
: 2438 \\
: 3321 \\
: 3062 \\
: 3832 \\
: 2687 \\
: 4210 \\
: 2094 \\
: 3750 \\
: 2356 \\
: 3347 \\
: 3166 \\
: 1940 \\
: 3597 \\
: 2534 \\
: 4213 \\
: 2927 \\
: 3801 \\
: 2401 \\
: 3336 \\
: 2968 \\
: 1970 \\
: 3782 \\
: 2576 \\
: 4204 \\
: 3104 \\
: 3489\end{array}$ & 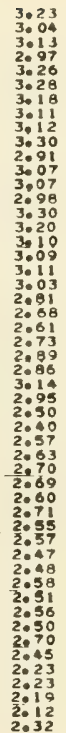 & 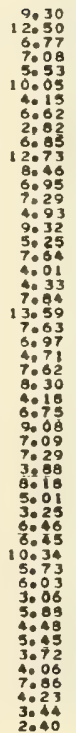 & 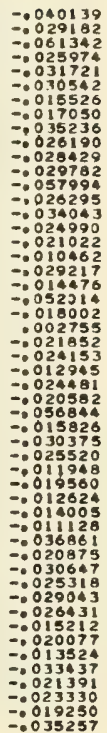 \\
\hline
\end{tabular}

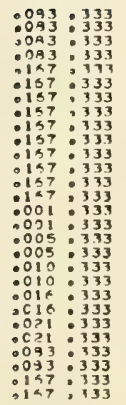

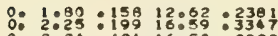

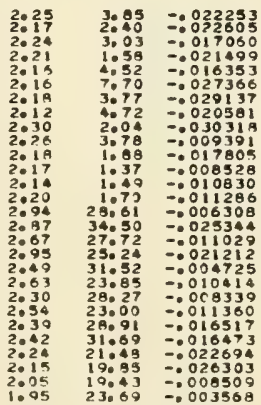





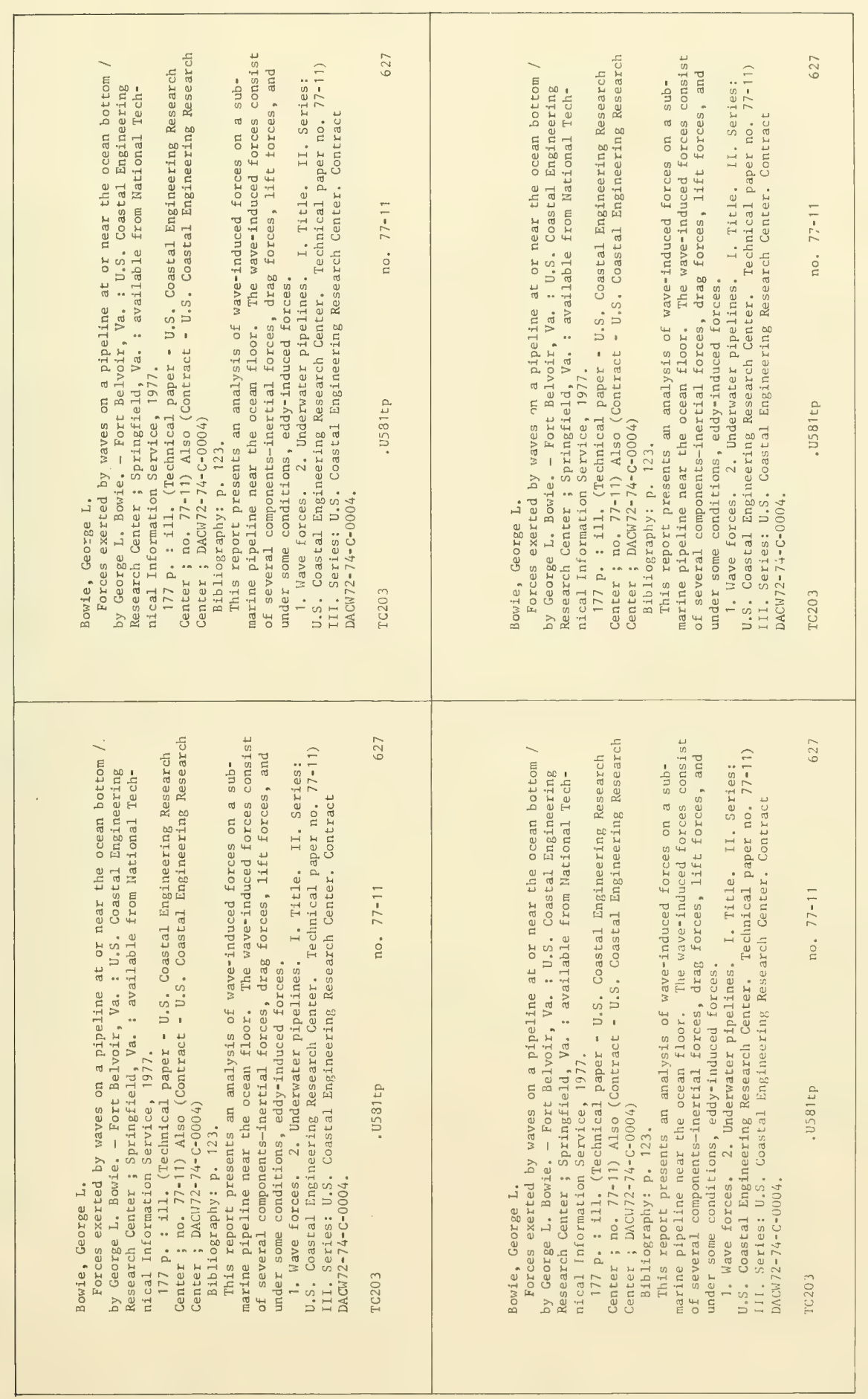





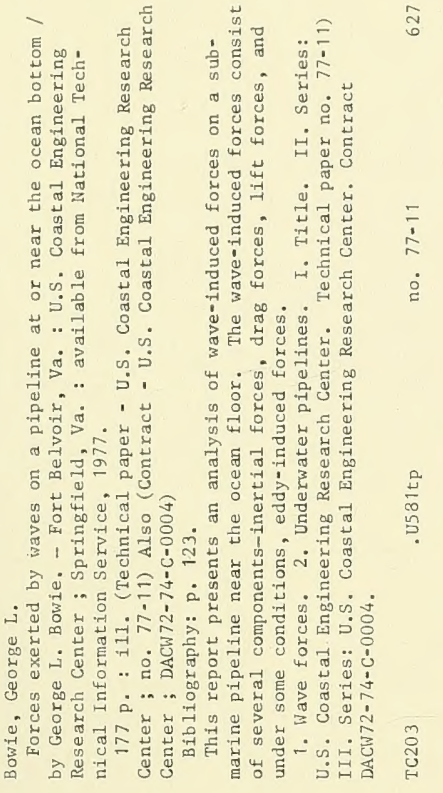

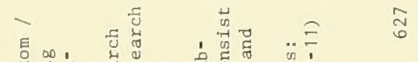

म. 万ु

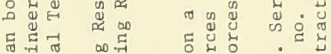

可

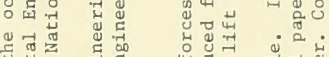

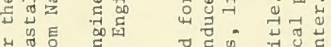

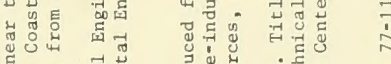

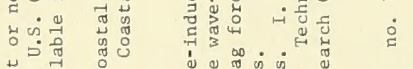

त...

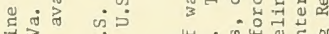

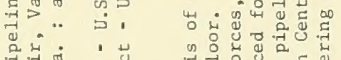

م्ञ

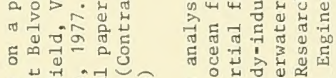
.

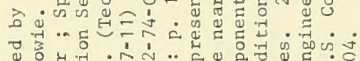

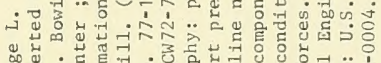

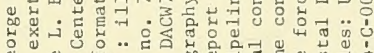

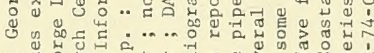

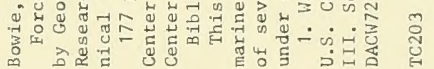

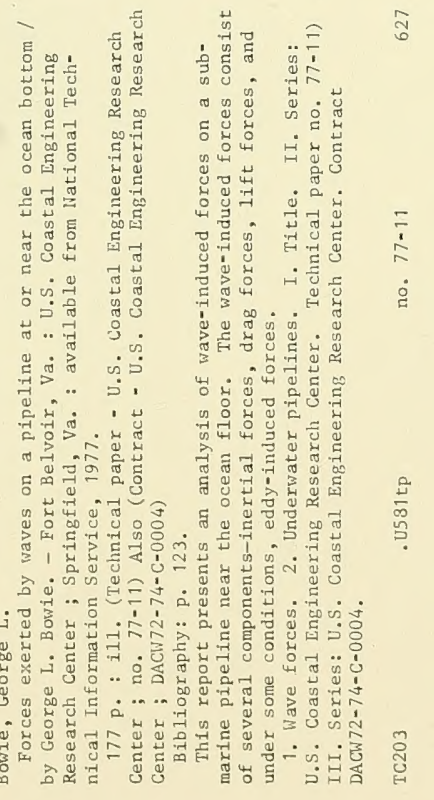

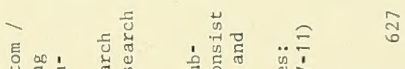

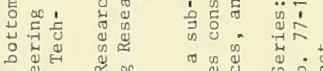

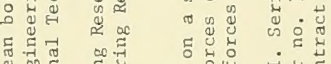

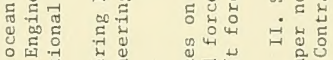

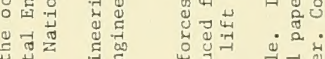

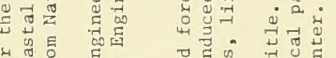

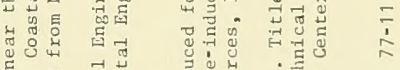

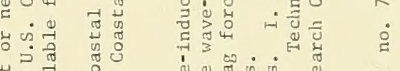

ब...

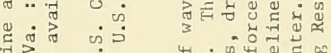

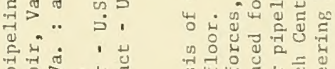

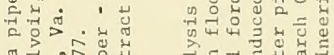

๙

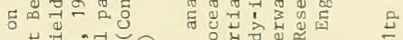

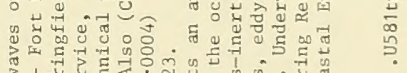

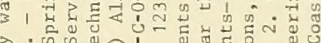

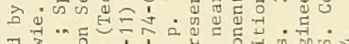

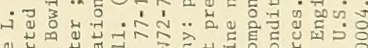

ơ

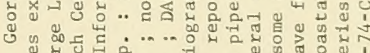

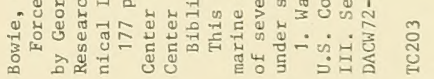



